\title{
Translational research on exhaled volatile organic compounds from bedside to bench
}

Citation for published version (APA):

Fijten, R. R. R. (2017). Translational research on exhaled volatile organic compounds from bedside to bench. [Doctoral Thesis, Maastricht University]. Maastricht University. https://doi.org/10.26481/dis.20171211rf

Document status and date:

Published: 01/01/2017

DOI:

10.26481/dis.20171211rf

Document Version:

Publisher's PDF, also known as Version of record

\section{Please check the document version of this publication:}

- A submitted manuscript is the version of the article upon submission and before peer-review. There can be important differences between the submitted version and the official published version of record.

People interested in the research are advised to contact the author for the final version of the publication, or visit the DOI to the publisher's website.

- The final author version and the galley proof are versions of the publication after peer review.

- The final published version features the final layout of the paper including the volume, issue and page numbers.

Link to publication

\footnotetext{
General rights rights.

- You may freely distribute the URL identifying the publication in the public portal. please follow below link for the End User Agreement:

www.umlib.nl/taverne-license

Take down policy

If you believe that this document breaches copyright please contact us at:

repository@maastrichtuniversity.nl

providing details and we will investigate your claim.
}

Copyright and moral rights for the publications made accessible in the public portal are retained by the authors and/or other copyright owners and it is a condition of accessing publications that users recognise and abide by the legal requirements associated with these

- Users may download and print one copy of any publication from the public portal for the purpose of private study or research.

- You may not further distribute the material or use it for any profit-making activity or commercial gain

If the publication is distributed under the terms of Article $25 \mathrm{fa}$ of the Dutch Copyright Act, indicated by the "Taverne" license above, 


\section{TRANSLATIONAL RESEARCH ON EXHALED VOLATILE ORGANIC COMPOUNDS FROM BEDSIDE TO $\mathrm{BENCH}$}

Rianne R.R. Fijten 
(C) Rianne R.R. Fijten, 2017

Translational research on Volatile Organic Compounds from bedside to bench

ISBN: 978-94-6233-822-7

Cover Design: René Fijten

Layout: Rianne Fijten

Printed by: Gildeprint, Enschede

The research presented in this dissertation was conducted at NUTRIM School of Nutrition and Translational Research in Metabolism of Maastricht University 


\title{
TRANSLATIONAL RESEARCH ON EXHALED VOLATILE ORGANIC COMPOUNDS FROM BEDSIDE TO $\mathrm{BENCH}$
}

\author{
PROEFSCHRIFT
}

ter verkrijging van de graad van doctor aan de Universiteit Maastricht, op gezag van de Rector Magnificus, Prof. Dr. Rianne M. Letschert volgens het besluit van het College van Decanen in het openbaar te verdedigen op maandag 11 December 2017 om 14:00 uur

door

Rianne Rebecca Roger Fijten 


\section{Promotor}

Prof. dr. F.J. van Schooten

\section{Co-promotores}

Dr. A. Smolinska

Dr. A.W. Boots

\section{Beoordelingscommissie}

Prof. dr. A. Bast (voorzitter)

Dr. R. Cavill

Prof. dr. E. Dompeling

Dr. H.C. Keun (Imperial College, United Kingdom)

Prof. dr. P.J. Sterk (University of Amsterdam) 
Chapter 1 Introduction $\quad 9$

1.1 Volatile Organic Compounds . . . . . . . . . . . . . . . . . 10

1.2 Exhaled Breath . . . . . . . . . . . . . . . . . . . . . . . . . . . . . . . .

1.3 Omics . . . . . . . . . . . . . . . . . . . 24

1.4 The aims of this thesis . . . . . . . . . . . . . 26

Chapter 2 Current Breathomics - a review on data pre-processing techniques and machine learning in metabolomics breath analysis 39

2.1 Abstract . . . . . . . . . . . . . . . . . 40

2.2 Introduction . . . . . . . . . . . . . . . . . . . 40

2.3 Instrumentation . . . . . . . . . . . . . . . . . . . . . . . . . . . . . . . . . . . . . . .

2.4 Data pre-processing . . . . . . . . . . . . . . . . . . . 44

2.5 Normalization, scaling and transformation . . . . . . . . . . . . 50

2.6 Multivariate statistical methods . . . . . . . . . . . . . . . . 52

2.7 Data fusion . . . . . . . . . . . . . . . . . . . . . . . . . . . . . 63

2.8 Validation of multivariate statistical models . . . . . . . . . . . . 65

2.9 Summary . . . . . . . . . . . . . . . . . . 68

Chapter 3 Analysis of volatile organic compounds in exhaled breath $\begin{array}{ll}\text { to diagnose ventilator-associated pneumonia } & \mathbf{7 7}\end{array}$

3.1 Abstract . . . . . . . . . . . . . . . . 78

3.2 Introduction . . . . . . . . . . . . . . . . . . . . . 78

3.3 Materials and Methods . . . . . . . . . . . . . . . . . . . 79

3.4 Results . . . . . . . . . . . . . . . . . . . . . 83

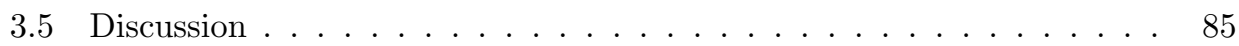

3.6 Conclusion . . . . . . . . . . . . . . . . . . . . . . . 89

3.7 Supplementary Materials . . . . . . . . . . . . . . . . . 90 
Chapter 4 The use of exhaled air analysis in discriminating interstitial lung diseases: a pilot study

4.1 Abstract . . . . . . . . . . . . . . . . 96

4.2 Introduction . . . . . . . . . . . . . . . . . . . . . . . . . . . . . . . . . . . .

4.3 Materials \& Methods . . . . . . . . . . . . . . . . . . . . . . . . . . . . . . . . . . . . . . . . . . . . .

4.4 Results . . . . . . . . . . . . . . . . . . . 103

4.5 Discussion . . . . . . . . . . . . . . . . . . . . . 108

4.6 Supplementary Materials . . . . . . . . . . . . . . . . . 113

Chapter 5 The necessity of external validation in exhaled breath research: a case study of sarcoidosis

5.1 Abstract . . . . . . . . . . . . . . . . . 122

5.2 Introduction . . . . . . . . . . . . . . . . . . . . 122

5.3 Materials and Methods . . . . . . . . . . . . . . . . . . . . . 124

5.4 Results . . . . . . . . . . . . . . . . . . . 127

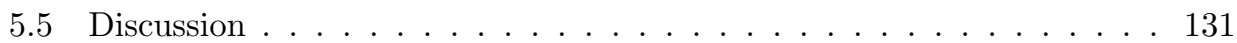

Chapter 6 Identification of microorganisms based on headspace analysis of volatile organic compounds by gas chromatographymass spectrometry

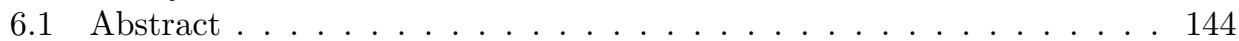

6.2 Introduction . . . . . . . . . . . . . . . . . . . . 144

6.3 Materials and Methods . . . . . . . . . . . . . . . . . . 146

6.4 Results . . . . . . . . . . . . . . . . . . . . . 149

6.5 Discussion . . . . . . . . . . . . . . . . . 153

Chapter 7 Exposure to genotoxic compounds alters in vitro cellular VOC excretion $\quad 165$

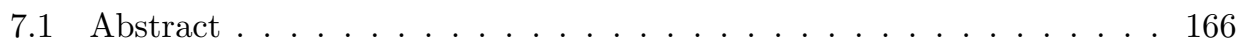

7.2 Introduction . . . . . . . . . . . . . . . . . 166

7.3 Materials and Methods . . . . . . . . . . . . . . . . . . 167

7.4 Results . . . . . . . . . . . . . . . . . . . 170

7.5 Discussion \& Conclusion . . . . . . . . . . . . . . . . . . . . 172

7.6 Supplementary Materials . . . . . . . . . . . . . . . . . 174

Chapter 8 Determining the endogenous sources of Volatile Organic Compounds excreted after benzo[a]pyrene exposure in vitro 181

8.1 Abstract . . . . . . . . . . . . . . . . . . . . . . . . . . . . . . . . . . . . . . . .

8.2 Introduction . . . . . . . . . . . . . . . . . . . . . 182

8.3 Materials \& Methods . . . . . . . . . . . . . . . . . . . . . . 184

8.4 Results . . . . . . . . . . . . . . . . . . . . . 187

8.5 Discussion \& Conclusion . . . . . . . . . . . . . . . . . . . . . . 191

8.6 Supplementary Materials . . . . . . . . . . . . . . 197

Chapter 9 Summary \& Discussion 209

9.1 From bedside to bench . . . . . . . . . . . . . . . . . . 210

9.2 General discussion . . . . . . . . . . . . . . . . . 210 
9.3 Conclusion and future perspectives . . . . . . . . . . . . . . . 219

9.4 Main Findings . . . . . . . . . . . . . . . . . . . . . . . . . . 219

$\begin{array}{ll}\text { Nederlandse Samenvatting } & 231\end{array}$

$\begin{array}{ll}\text { Valorisatie-addendum } & 237\end{array}$

$\begin{array}{ll}\text { Dankwoord } & 241\end{array}$

$\begin{array}{ll}\text { Curriculum Vitae } & 245\end{array}$

$\begin{array}{lr}\text { List of Publications } & 247\end{array}$ 

CHAPTER 1

Introduction 


\subsection{Volatile Organic Compounds}

Volatile organic compounds (VOCs) are defined as carbon-containing molecules exhibiting a high vapor pressure at room temperature. They are divided in several chemical classes such as alkanes, aldehydes, alcohols, ketones, esters, amines and aromatic hydrocarbons [1]. Thousands of different VOCs have been identified that originate from endogenous sources such as the human body or from bacteria and from exogenous sources like pollution, plants, food, cosmetics and fungi. Since changed concentrations of VOCs may be related to disrupted metabolic processes in the human body, there is a promise that they may act as noninvasive biomarkers of health and disease. Since exhaled VOCs are originating from the pulmonary tract, or alternatively have gone through the lungs coming from the circulating blood, they are candidate biomarkers in the diagnosis and monitoring of various diseases, and especially lung diseases.

\subsubsection{Endogenous VOCs}

Endogenous VOCs can be produced by either the human body itself or both commensal and pathogenic bacteria present in the human body.

Extensive research has been performed on the VOCs emitted by bacteria in vitro $[2-6]$. The VOCs that are measured in these studies are emitted in the air above the bacteria, also known as the headspace. These VOCs are then captured and analysed by mass spectrometry (MS). Bos et al. has systematically reviewed the in vitro studies concerning VOCs emitted by six different strains of bacteria, i.e. Staphilococcus aureus, Streptococcus pneumoniae, Enterococcus faecalis, Pseudomonas aeruginosa, Klebsiella pneumoniae and Escherichia coli. They concluded that specific VOCs that are exclusively excreted by individual bacterial strains [7], such as excretion of methyl-thiocyanide, 1-undecene, hydrogen cyanide and 4-methylquinazoline by Pseudomonas aeruginosa and methanol and pentanol by Escherichia coli. A larger group of VOCs, including isoprene and formaldehyde and ethanol, was emitted by all bacterial strains examined. These findings suggest that certain sets of VOCs will allow for discrimination between specific strains whereas others may allow for distinction between the presence or absence of a bacterial infections. Differentiation between different bacterial infections in vivo has not yet been investigated.

Little is known about the endogenous production of VOCs by the human body itself. It is hypothesized that VOCs are produced as by-products of normal cellular processes in the body [1]. These cellular processes are altered when disease occurs, resulting in a modified production of VOCs. Upon formation in a healthy or diseased organ, VOCs are able to diffuse from the cell's cytoplasm to the bloodstream due to their high volatility [8]. When they arrive at the lungs, they will diffuse through the walls of the blood vessels into the alveolar air, which is then exhaled. Additionally, VOCs produced in the lungs can directly diffuse into the exhaled breath depending on the exact location of their formation [8].

The endogenous source of only a handful of VOCs has been reported thus far. For instance, dimethylsulfide is responsible for fetor hepaticus, also known as breath of the dead, a late sign of liver failure [9, 10], whereas acetone is exhaled during ketoacidosis as a result of diabetes and sepsis [11-13]. 
Additionally, isoprene is produced in the human body as a by-product of the mevalonate pathway, which produces cholesterol and vitamin $K$ [14]. It has been linked to exercise and activity $[14,15]$, where the isoprene levels positively correlate to blood cholesterol levels [16] and are decreased during disease-related inactivity, in for instance acute respiratory distress syndrome and cystic fibrosis (CF) [17-20]. Additionally, atorvastatin therapy decreases blood cholesterol levels and exhaled isoprene levels in parallel, suggesting great potential for monitoring cholesterol status using exhaled isoprene [16]. Moreover, exhaled isoprene levels are elevated in men and lower in younger people [21].

For many other VOCs, the endogenous sources have been hypothesized, yet not proven. Hydrocarbons such as pentane and ethane are thought to be produced during two processes called oxidative stress and lipid peroxidation [22]. Oxidative stress is defined as a disturbance in the balance between the production of reactive oxygen species (ROS) and the presence of antioxidant defenses in favor of the former [23], resulting in the oxidation and degradation of cell membrane lipids, which is called lipid peroxidation [24]. The hypothetical sources of aldehydes such as decanal and nonanal are normal physiological processes including gene regulation, proliferation and signal transduction $[1,25]$. They are for instance generated by the biotransformation of amino acids, neurotransmitters, carbohydrates and lipids, but also arise from nonphysiological damaging processes such as lipid peroxidation [25]. Examples of the latter include 3-hydroxy-2-nonenal and malondialdehyde [26].

The evidence above demonstrates that, although some VOCs may be solely produced by either exogenous or endogenous sources, many are the result of both. For instance, isoprene is produced in large quantities by plants and humans, whereas hydrocarbons and aldehydes are produced by humans, plants and pollution. Therefore, it is important to investigate which VOCs can be of value for disease diagnosis of exhaled breath by studying their endogenous sources.

\subsubsection{Exogenous VOCs}

VOCs derived from exogenous sources can be divided into two categories: originating from nature and related to pollution [27]. Methane is the most abundantly emitted exogenous VOC, with a global yearly emission of 8000 teragrams of carbon ( $\mathrm{tg}-\mathrm{C} / \mathrm{yr}$ ), which accounts for approximately $10 \%$ of all greenhouse gases emissions [28]. The emission of VOCs other than methane (a.k.a. non-methane VOCs; NM-VOCs) adds up to $1273 \mathrm{tg}-\mathrm{C} / \mathrm{yr}$, of which $69 \%$ is emitted by natural sources, whereas the other $31 \%$ is emitted as a result of pollution [27]. The two largest natural sources of VOCs are vegetation and oceanic production, whereas pollution-related VOCs are mainly produced by biomass burning and transportation [29].

More than 1700 compounds produced by vegetation are known to be volatile [30]. Many of these NM-VOCs protect plants from stress and aid the communication between plants and with insects. They include terpenoids, green leaf volatiles, aromatic compounds and sulfur- and nitrogen-containing VOCs [30-33]. The terpenoid isoprene is the most abundantly emitted NM-VOC, represented by $44 \%$ of natural emissions, and offers protection against ozone and free radicals [34, 35]. Monoterpenes serve the same purpose and account for $11 \%$ of the natural NM-VOC emis- 
sions $[27,35]$.

Of the VOCs emitted by pollution, the main source is burning of biomass, either man-made or natural. Biomass burning releases many different VOCs of which the composition changes at different stages of burning [36]. During the initial heating stage, with temperatures typically $<100^{\circ} \mathrm{C}$ ), aromatic hydrocarbons, ethers, light aldehydes and acetic acid are mainly emitted, whereas at temperatures between 250 and $500^{\circ} \mathrm{C}$ a variety of VOCs are released, including (heavier) aldehydes, furanes and aromatic compounds such as benzene, toluene and phenol [36]. In the final smoldering phase, the emission of VOCs is low and mainly includes $\mathrm{CO}_{2}$ [36]. The other sources of pollution-related VOCs include industry and traffic, of which the latter is the main source of air pollution in urbanized areas [37]. VOCs emitted by these sources include alkanes such as heptane and hexane, aldehydes such as acetaldehyde and formaldehyde, and aromatic hydrocarbons such as toluene and styrene [38].

\subsection{Exhaled Breath}

Exhaled breath consists of a mixture of nitrogen, oxygen, carbon dioxide, noble gases [39]. In addition to this mixture of gases, VOCs are present in concentrations that range from nmol/l to pmol/l [39, 40]. Due to its non-invasive nature and the potential for rapid diagnosis, exhaled breath analysis has showed promise as a diagnostic tool. Additionally, since exhaled breath collection does not require effort, it can be applied to children, the elderly or patients with severe respiratory impairments [41].

Exhaled breath can be sampled, measured and analyzed with a variety of sampling methods, analytical platforms and statistical techniques. With respect to the collection of exhaled breath, three methods are commonly used. The first method is called upper airway collection and samples only the dead space air, which is approximately one third of the air present in the lungs at any given time [42]. This method has been used previously for nitric oxide measurements in the dead space [43]. Second, alveolar air collection ensures that VOCs are only collected from the area where gas exchange takes place, as the dead space simply dilutes the VOCs that are excreted into the alveolar air [44]. For this reason, this is the preferred lung compartment to sample, especially for off-line sampling and when measuring endogenously formed VOCs. However, sophisticated equipment is needed to exclude the dead space air. Third, mixed air sampling collects both the alveolar and the dead space air. In contrast to alveolar air collection, no sophisticated equipment is needed, making this method is more simple and more suitable for large-scale sampling of exhaled breath.

Different devices are used to sample the exhaled breath. Electronic noses (eNoses) use sensors to detect changes in the exhaled breath and are suitable for clinical use due to their small size [45]. However, eNoses measure a broad range of VOCs in the exhaled breath without chemical identification of VOCs of interest, and is therefore less suitable for research purposes [46]. For that reason, mass spectrometry (MS) is widely used to study exhaled air as this technique allows for the chemical identification of individual VOCs that can also be combined into VOC profiles. The most widely used technique to measure and identifiy the content of exhaled air is gas chromatography - mass spectrometry (GC-MS), followed by proton transfer reaction mass 
spectrometry (PTR-MS), selected-ion flow-tube - mass spectrometry (SIFT-MS) and ion mobility spectrometry (IMS) [47, 48]. The advantages and disadvantages of the different MS-based analytical platforms for measuring exhaled breath have been summarized previously [49]. The largest general disadvantage of MS is that the machines are large, expensive and require skilled personnel, which makes them less suitable for clinical use than an eNose device.

Since the lungs are in direct contact with the exhaled breath, they are a suitable candidate to examine the value of exhaled breath analysis. The research presented in this thesis deals with the application of VOCs analysis in respiratory related disease and for that reason, only lung diseases will be discussed below.

\subsubsection{Study options}

The last several decades, the focus of studying exhaled breath has been on investigating its diagnostic potential in human subjects due to its non-invasive nature. More recently, animal studies have been included as they allow for stricter controlling of possible confounders such as age, gender, dietary habits and environmental influences. Additionally in the last few years, in vitro studies have become more popular, which generally attempt to identify associations between cellular processes and specific VOCs excreted by cells.

\subsubsection{Human studies}

The potential of exhaled breath has been investigated in a multitude of lung diseases, with the majority of studies investigating lung cancer. Other diseases include (childhood) asthma, chronic obstructive pulmonary disease (COPD), tuberculosis (TB), cystic fibrosis (CF), ventilator-associated pneumonia (VAP), and interstitial lung disease (ILD). An overview of the scientific studies on exhaled breath based on VOC profiles using mass spectrometry in these lung diseases can be found in table 1.1. A total of 88 studies were included, with $50 \%$ investigating lung cancer. The number of included participants ranged from 9 to 661 per study. Thirteen studies did not include controls as their objective was to distinguish between patients with different diseases stages or at different time points [6, 41, 50-60]. For instance, Fowler et al. [60] collected exhaled breath samples of VAP patients over time and found time-dependent VOC patterns that may correspond to the disease prognosis. On the other hand, Nardi-Agmon et al. [51] compared patients with stable versus progressive lung cancer and observed significant alteration in exhaled styrene levels.

The number of reported discriminatory VOCs varied between studies from 1 to 500. However, not all studies could report the number of VOCs used to discriminate between different groups as they used the whole range of measured VOCs rather than a selected subset. This is for instance true for the study by Phillips et al., which reported a model based on 500 VOCs [61].

Six percent of the studies did not report specifics on statistics or did not perform statistics at all. Univariate statistics is the simplest form of statistics that takes into account one variable at a time [62]. For breathomics, univariate statistics will test whether one VOC is different between two groups of participants. Examples of uni- 
variate statistical methods are the Student's t-test and Mann Whitney-U test [62, 63]. From the investigated studies, $48 \%$ applied univariate statistics, where generally a pvalue of less than 0.05 is considered significant. The problem with this is that each p-value calculation has a $5 \%$ chance that it is significant by chance, implying that when thousands of univariate tests are calculated on a dataset, there is a high likelihood that random events appear as significant. Multiple testing correction (MTC) methods are used to reduce the chance that false positives or negatives are generated in this way [64]. Consequently, applying MTC with univariate statistics is essential. Unforunately, MTC was only applied in $20 \%$ of studies that used univariate statistics.

The remaining $46 \%$ employed multivariate statistics, ranging from linear methods such as Partial Least Squares - Discriminant Analysis (PLS-DA) to non-linear methods like Random Forests (RF) $[65,66]$. The advantage of multivariate over univariate statistics is that it can analyze thousands of variables at the same time instead of one by one and does not require MTC $[63,67]$. The application of multivariate statistics allows for finding and investigating the relations between thousands of VOCs present in exhaled breath.

The final parameter mentioned in table 1.1 is study validation. This concerns validation of the discriminatory VOCs observed in a study to investigate the their validity, repeatability and reproducibility in other studies. Different validation methods exist and can be divided into internal and external validation. In the studies reported in table 1.1, $52(59 \%)$ did not report any use of validation. Internal validation was employed in 34 studies (39\%), and independent external validation was performed in only 2 studies $(2 \%)$.

The most used internal validation method was leave-one-out cross-validation (LOOCV; 12 studies) [51, 68-78], followed by k-fold cross-validation (KFCV; 6 studies) [41, 79-83]. Unfortunately, internal validation methods like LOOCV and KFCV do not give a realistic estimation of the predictive power of a statistical model [84] as only a small number or percentage of samples is used to test the validity of the predictive markers. The higher this percentage, the more reliable the estimate becomes [84]. Splitting of the data into a training set and a test set will provide a more reliable prediction, but splitting the data and subsequently creating a model many times with replacement (i.e. bootstrapping) provides the most realistic estimate of the true predictive power of a model [84]. Splitting of the data was used in 10 studies, including 5 studies where it was used in combination with another internal validation method [54, 61, 76, 85-89]. Yet, bootstrapping was used in only 3 studies [60, 90, 91].

For true external validation, a dataset is needed that differs from the original study by location or time [92]. For instance, the predictive model is created in a study performed at one hospital, and validated in a study performed at another hospital, but with a similar patient population [92]. External validation is the best measure of reproducibility of a clinical study [84], yet was only incorporated in two studies [93, 94]. In the first study, van Berkel et al. identified VOCs specific for COPD and validated those findings successfully in a new patient population sampled at a different location and time point [93]. The second study that incorporated external validation identified 5 VOCs as discriminatory between lung cancer patients and controls, and validated them in a study that sampled other lung cancer patients and controls two years later at the same location [94]. 


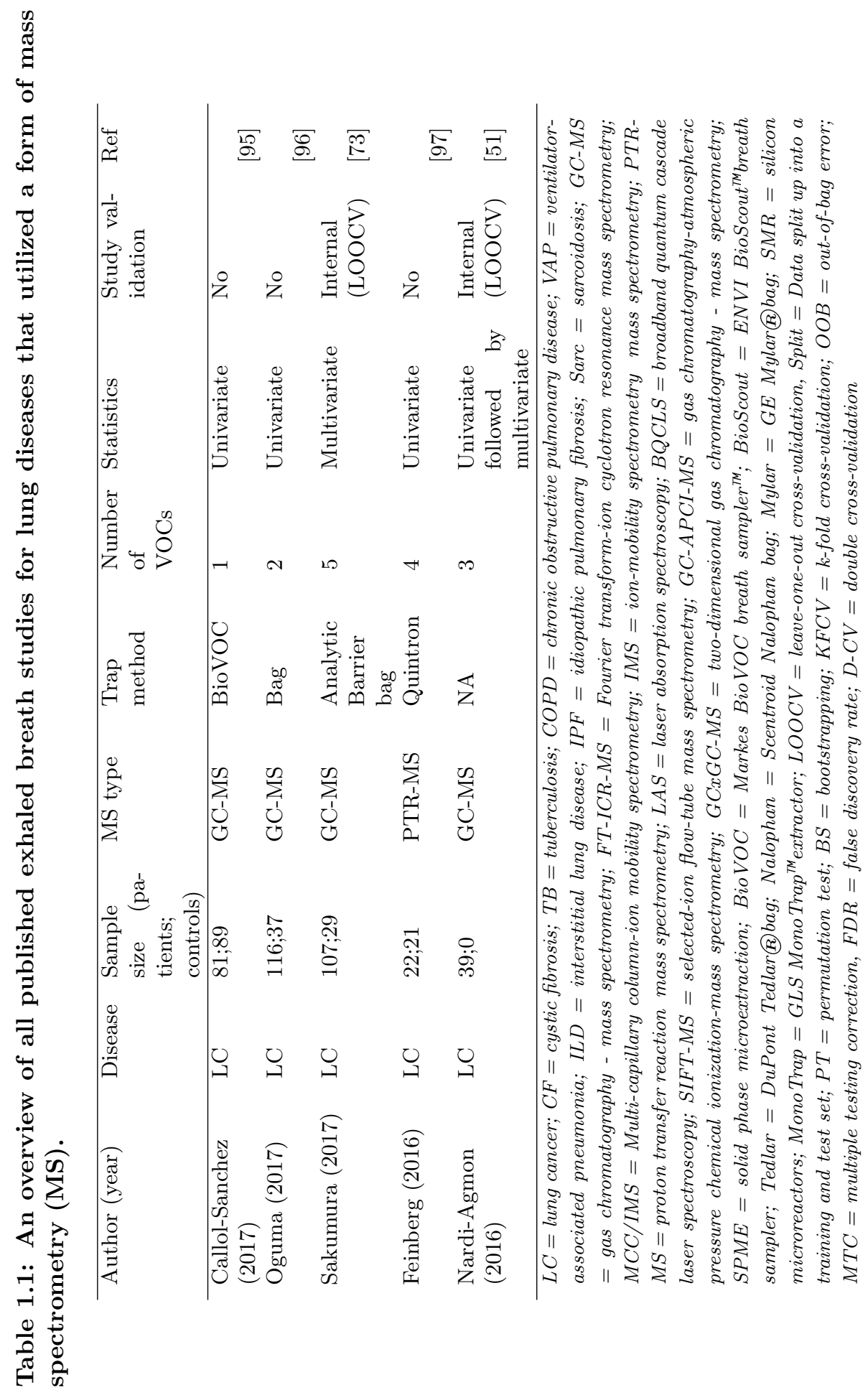




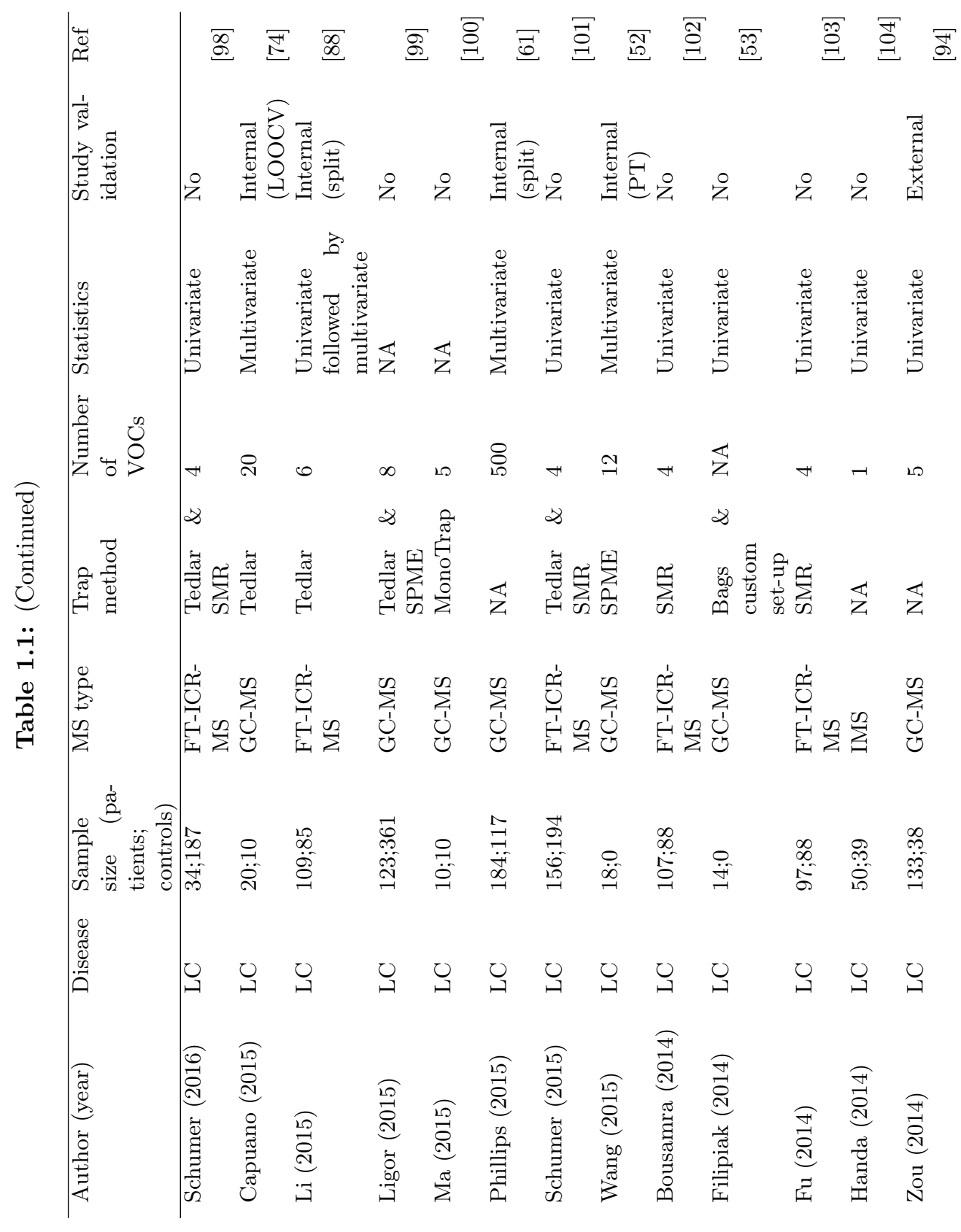




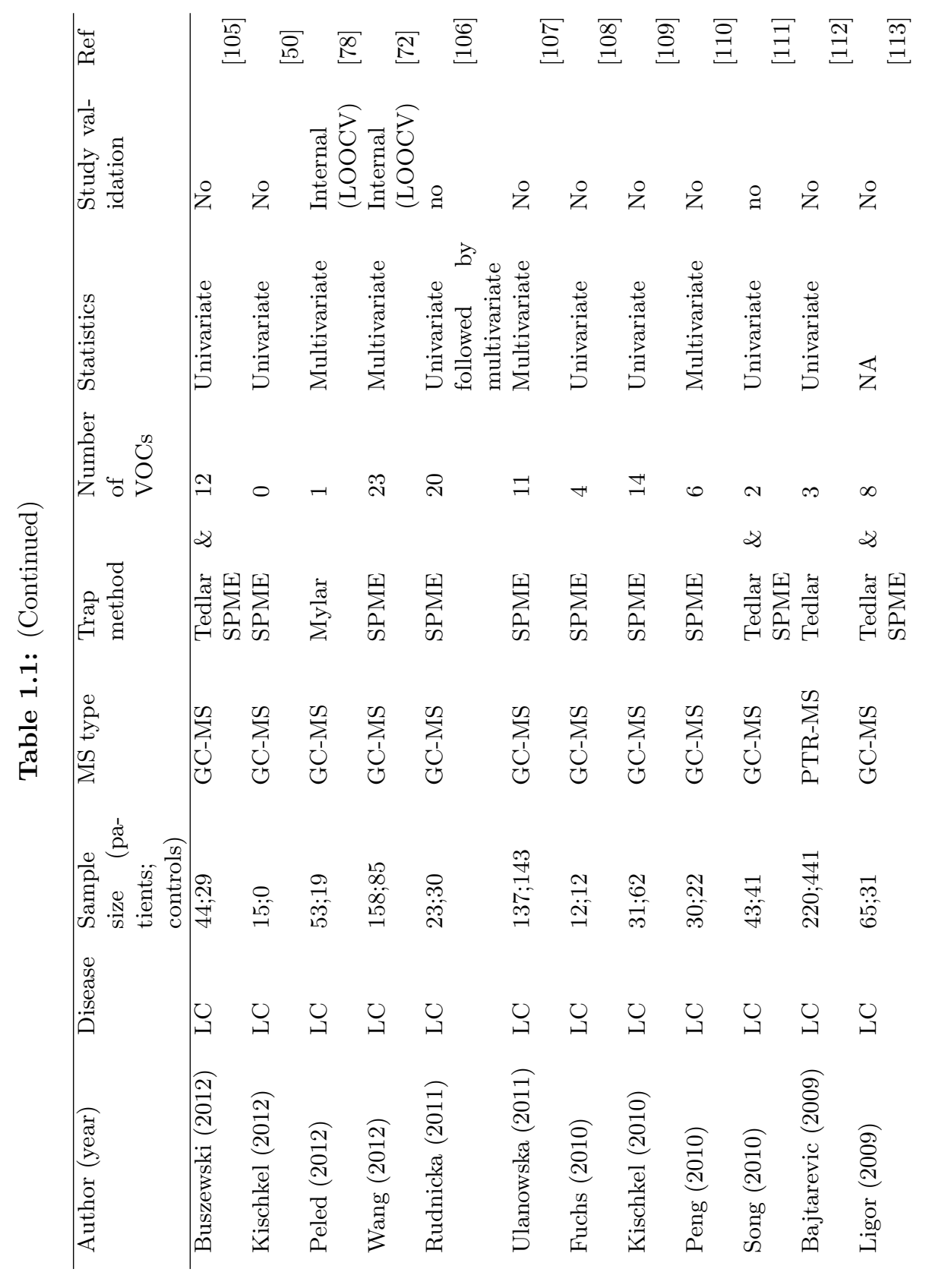




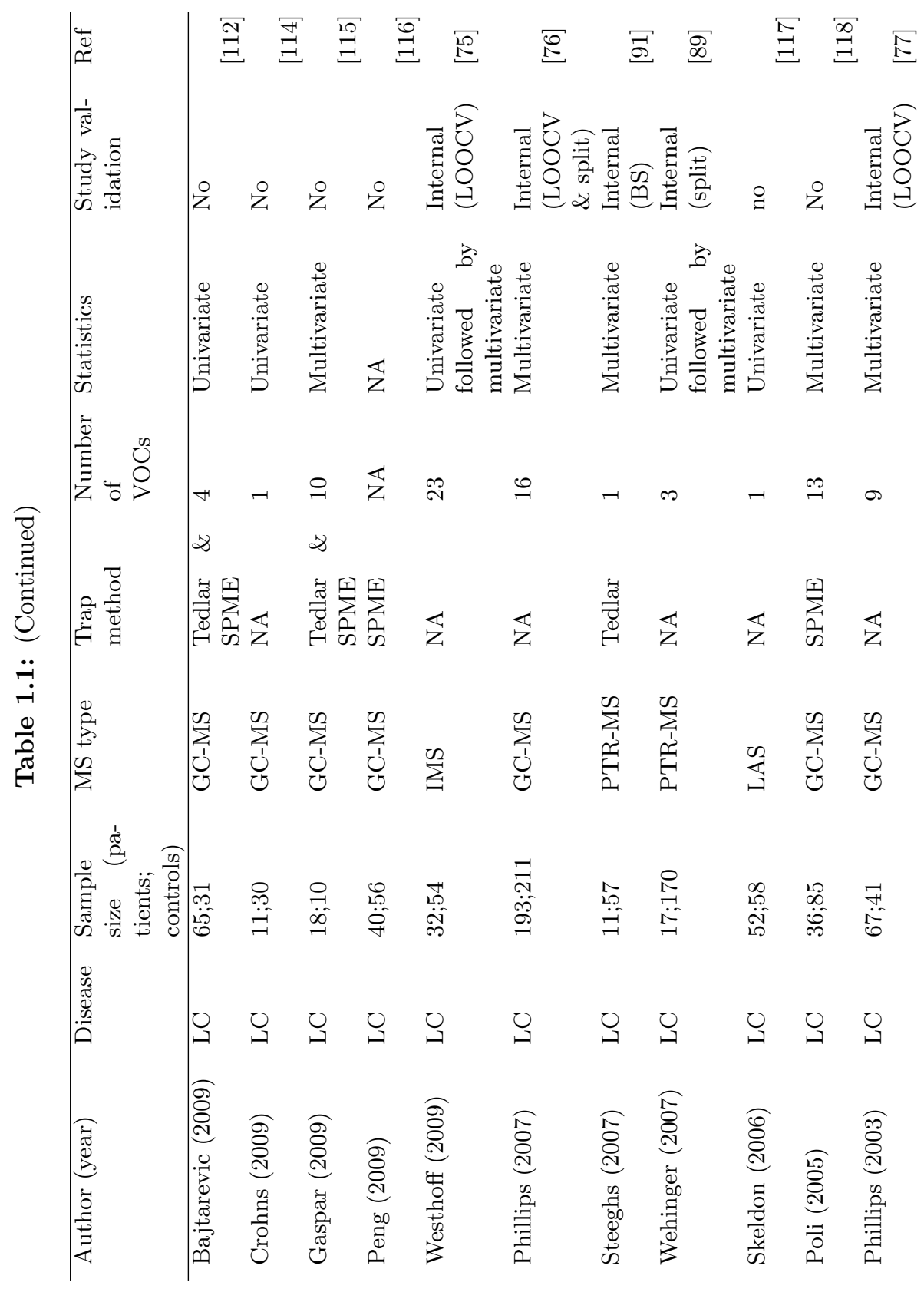




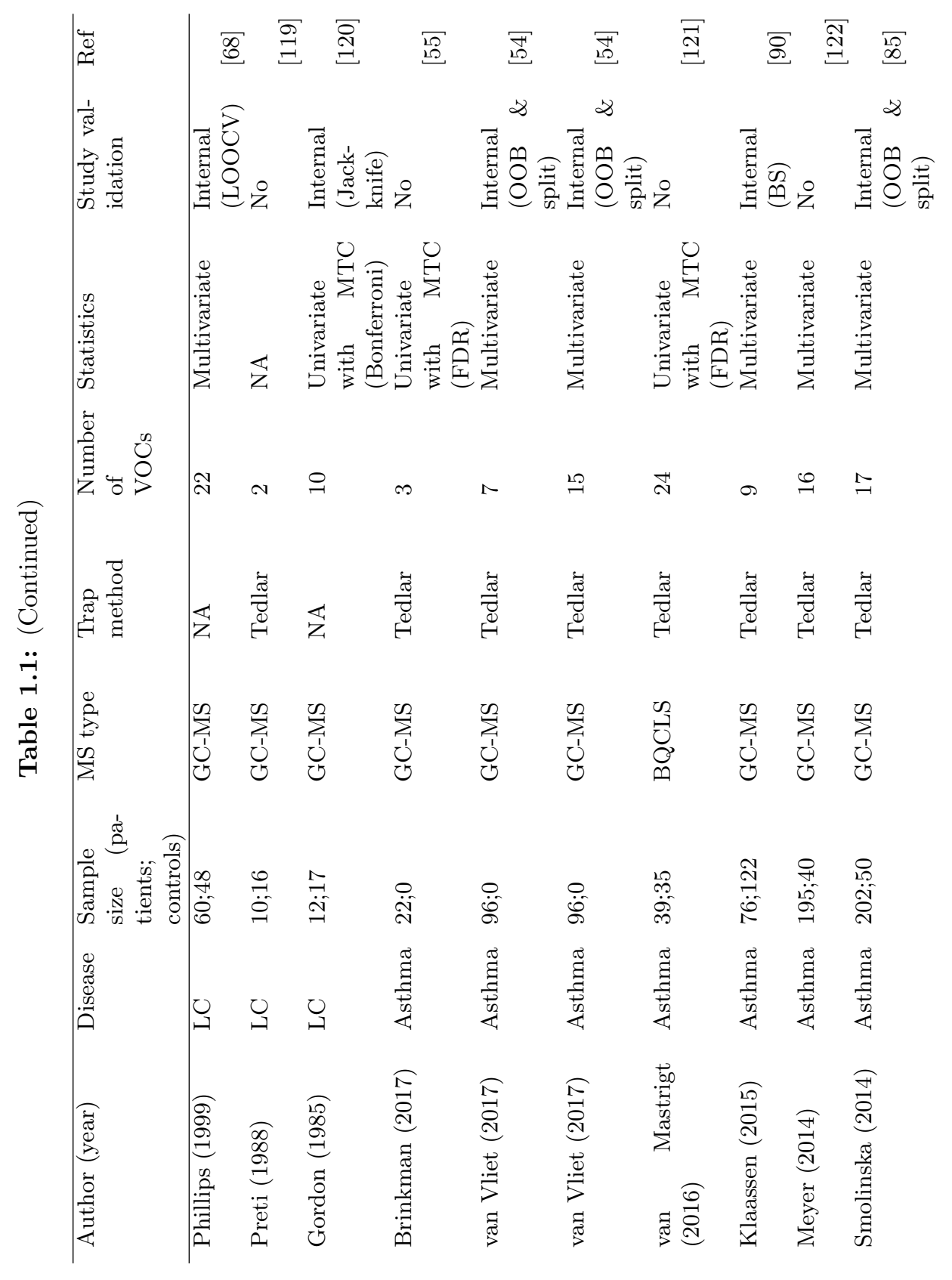




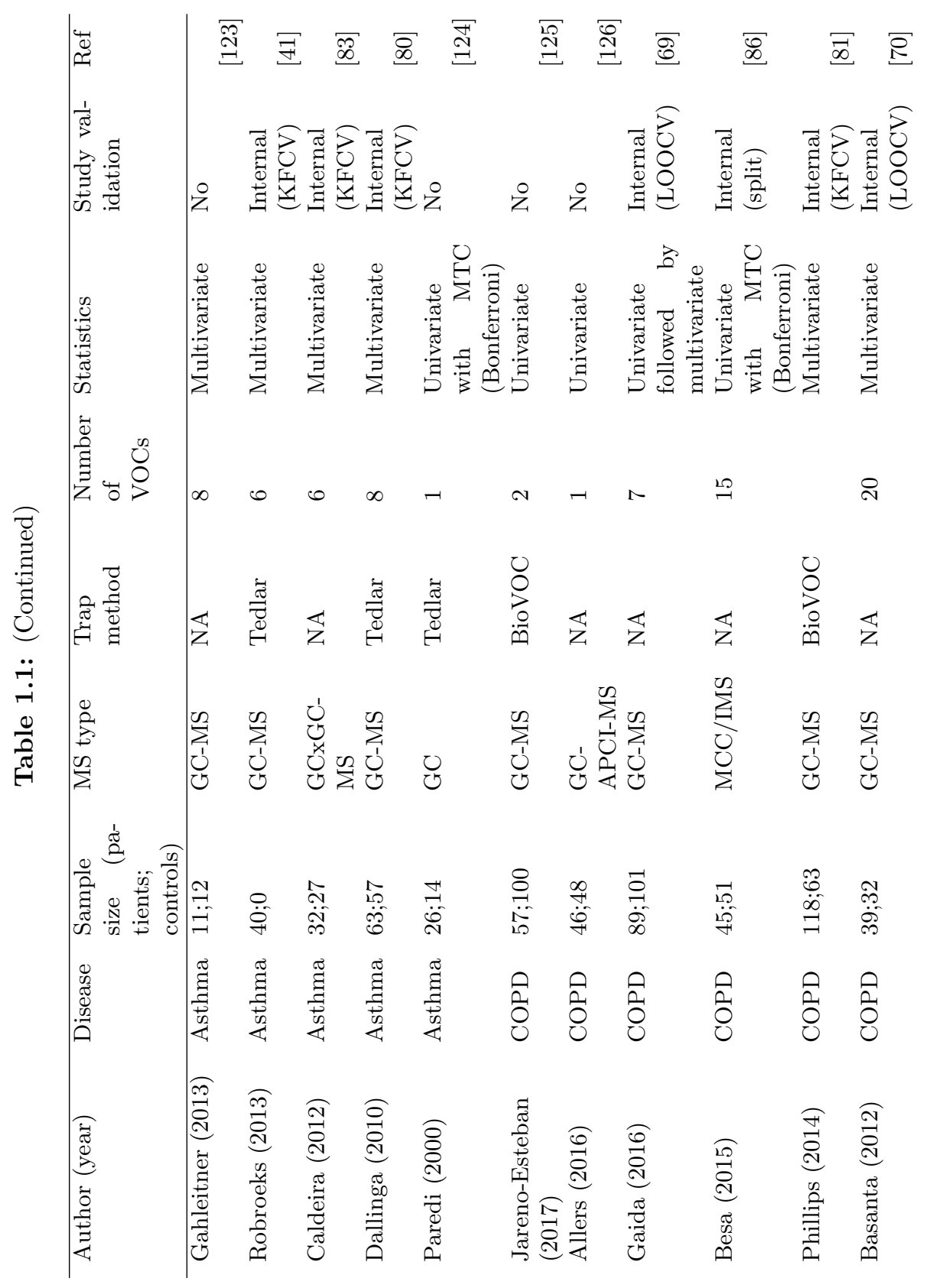




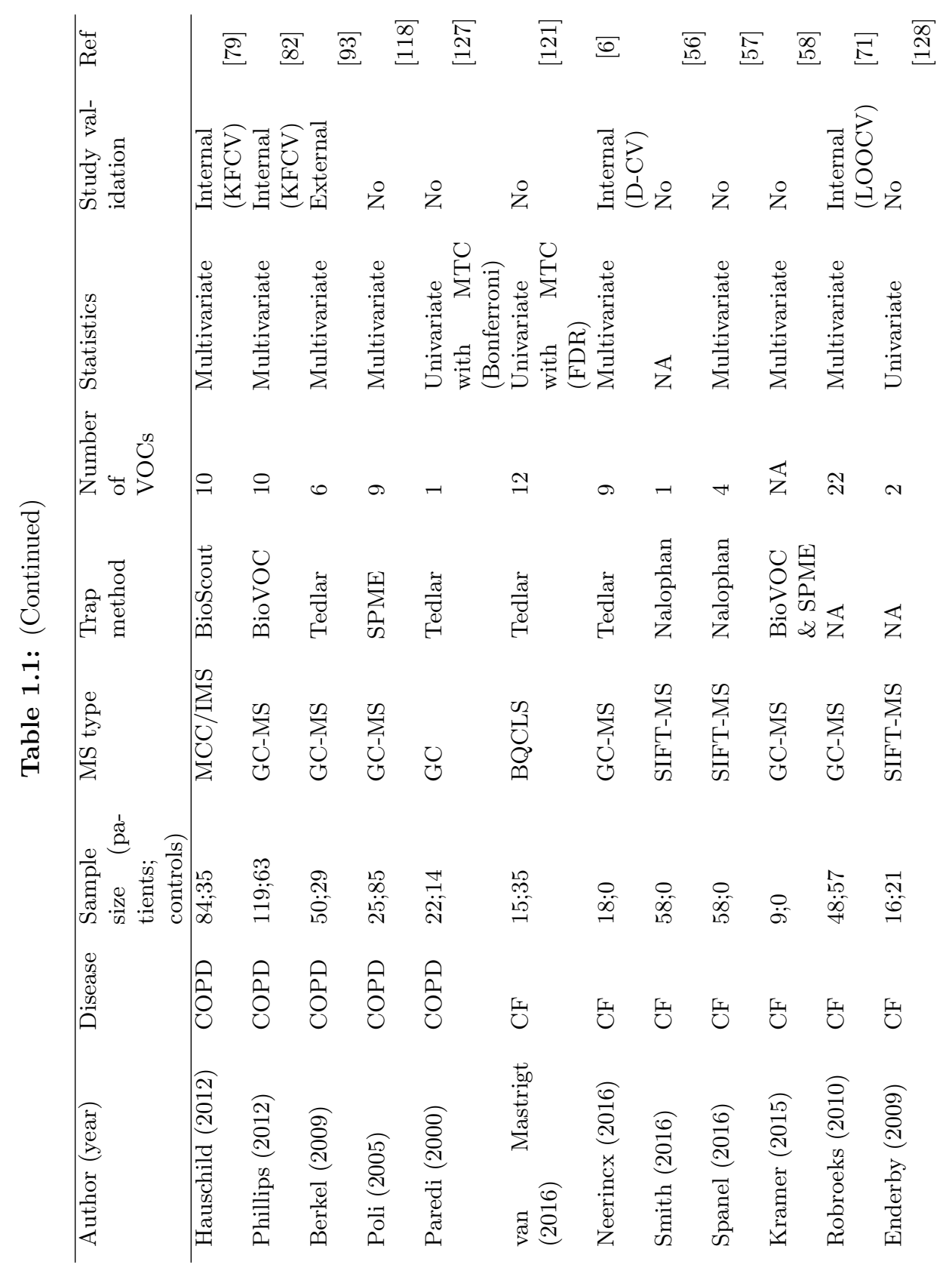




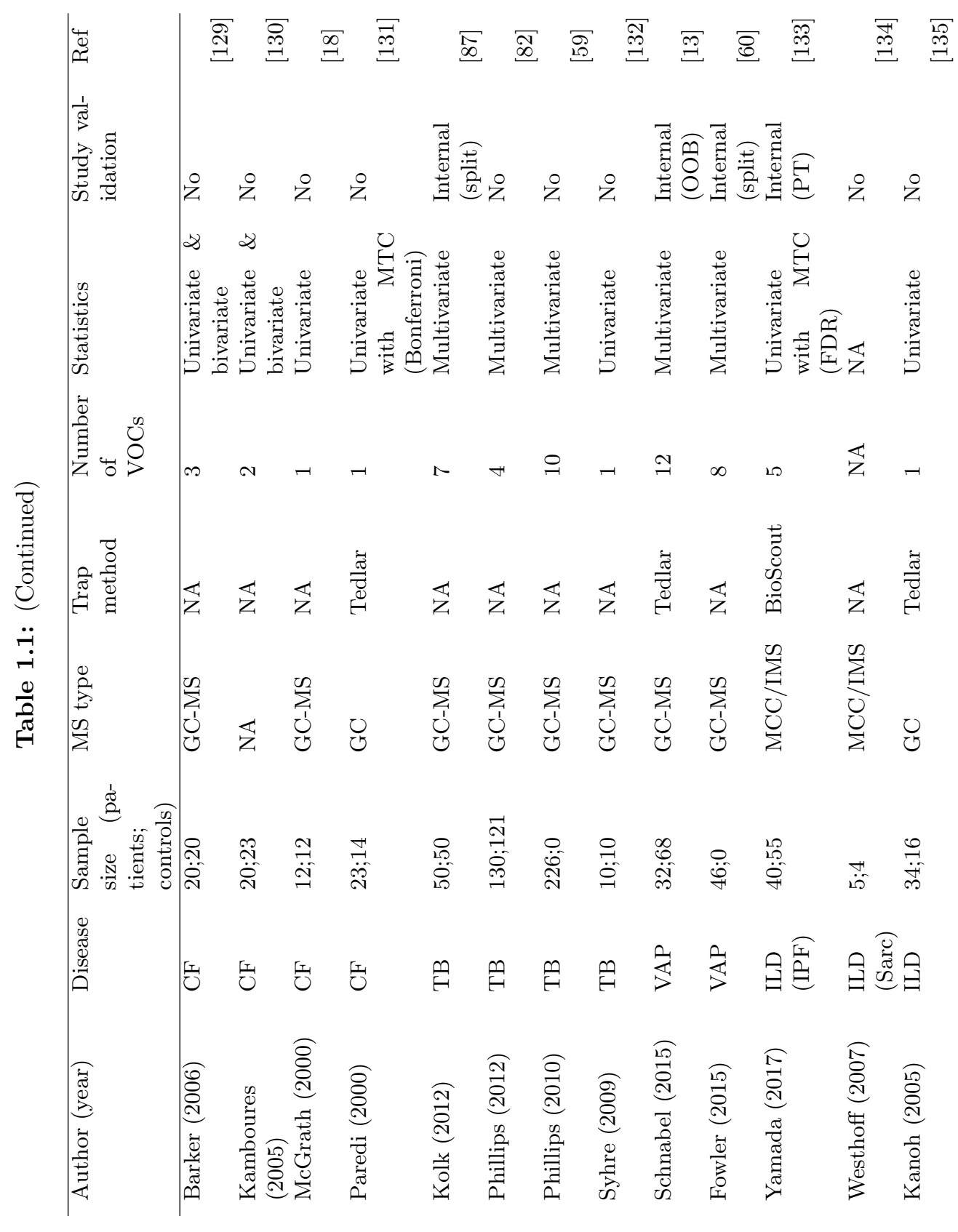




\subsubsection{Animal studies}

The composition of VOCs in the exhaled breath is very sensitive to different types of exogenous and endogenous factors such as pollution, dietary habits, hormonal changes, age, gender and BMI [136]. Controlling for all of these factors is difficult in human studies, yet possible in animal studies. Additionally, it allows researchers to study disease pathology and the influence of specific drugs on exhaled VOC profiles. Furthermore, animal studies can be used to model especially rare diseases, where acquiring a sufficient number of patients is challenging. Moreover, animal models are suitable for investigating the onset of diseases and can provide a more detailed insight in the underlying endogenous sources of VOCs.

Published animal experiments on exhaled breath analysis can be categorized into two categories: proof-of-principle methodology studies and disease-related studies. Methodological studies were performed on a variety of animal species including canines [137], horses [138], dolphins [139, 140], whales [141] and rodents [142]. Other studies have compared different sampling methods or examined the effect of confounders and found that the exhaled VOC profile was affected by different diets and sampling methods [142, 143].

Only a handful of studies were performed in animal models for lung diseases. Neuhaus et al. [144] mimicked inflammatory asthma in mice, but found no differences in the exhaled breath of healthy and diseased mice. Bos et al. [145] reported different VOC patterns after LPS-induced lung injury using anesthetized rats, but did not relate the reported volatiles to the damage inflicted. Another study examined allergic airway inflammation in anesthetized rodents and observed differences in the concentration of 3 exhaled VOCs between the healthy and diseased animals [146]. However, no chemical identification was performed and only 4 animals were used, indicating the need for a larger study to to confirm the usefulness of VOC analysis in allergic airway inflammation. Finally, Wyse et al. [147] examined airway inflammation in horses and found decreased levels of exhaled ethane and $\mathrm{CO}_{2}$ when airway inflammation was reduced by changing the stable bedding.

In general, two different methods are used to sample the exhaled breath of animals: (i) intubation during anaesthesia and (ii) while awake in a nose-only or head-only chamber. The advantage of the first method, which is used in most studies, is that the animals do not need to be trained and do not experience stress while collecting exhaled breath [145]. However, the downside of this approach is that the anaesthetic and its metabolites may be exhaled and thus influence the exhaled VOC profile [148]. In the second method, a rodent is placed in a chamber where only the head or nose sticks out and the air flows along the nose of the rodent, thereby transporting the exhaled VOCs to a capturing system $[142,149]$. As the animals are unable to move for a period of time during sampling, they may experience stress, which in turn may affect the composition of the exhaled VOCs [150]. To reduce the influence of stress on the exhaled breath, they are trained before the experiments start by spending increasing amounts of time in these chambers. The advantage of the nose-only set-up is that VOCs that are excreted by the skin, fur, faeces and urine are not detected, whereas it can be assumed that traces of VOCs excreted by the skin and fur will be picked up in the head-only set-up. However, no comparison between these two 
methodological variations has been performed.

In summary, whether to anaesthetize or not can influence the VOCs present in the sampled breath. Therefore, the appropriate method should be selected based on the aim of the study, the disease that is studied and the influence of the various pros and cons heron.

\subsubsection{In vitro studies}

Lately more and more studies are being published in which VOCs in the headspace air above in vitro cells cultures are sampled to investigate their endogenous production.

Several approaches can be used to examine excreted VOCs in the in vitro headspace and link these to endogenous cellular processes. The simplest approach is to measure headspace VOCs in flasks with cells and without cells to identify VOCs that are excreted during basal cellular metabolism. This was done in a variety of human cell culture experiments [151-156]. For instance Filipiak et al. found 4 and 11 VOCs with increased and decreased levels respectively while examining the human CALU-1 lung epithelial carcinoma cell line [151].

In addition, cells with different characteristics can be compared to identify VOCs that could be linked to those specific characteristics [157-162]. For example, Feinberg et al [160] sampled the headspace VOCs from 4 human lung cancer cell lines (A549, H2030, H358 and H322), which were divided into two groups based on their glycolytic profile. These groups could be distinguished by two VOCs of which the identity is unknown. Furthermore, the VOC profile was examined after addition of two glycolytic inhibitors to the cell culture, but was not influenced by these inhibitors.

Another option is to use a chemical trigger to induce a well-defined cellular response and measure VOCs in the headspace upon exposure to establish a plausible relationship between the excreted VOCs and the underlying pathways [163].

\subsection{Omics}

The detection of VOCs in breath via high-through put technologies has been named earlier breathomics and refers to the omics technologies that use a holistic approach to gain insight in disease etiology. Many omics fields exist, ranging from genomics, the large-scale study of the genome, and epigenomics, the large-scale study of the epigenome, to proteomics, the large-scale study of proteins, and metabolomics, the large-scale study of metabolites $[164,165]$. Each of these can provide insight in the biological processes underlying disease from a different perspective. The two omics of interest to this thesis are transcriptomics and metabolomics, which representatively identify genes or metabolites of interest to a specific disease or chemical.

\subsubsection{Transcriptomics}

Transcriptomics data is generally produced by microarrays or RNA sequencing and results in expression values for thousands of genes for a single sample [166]. This allows researchers to identify genes of interest. However, selecting individual genes from a list of thousands results in a large quantity of false-positive outcomes when 
using univariate statistical methods. For that reason, pathway analysis is used to combine the expression values of the individual genes into a more robust model using pre-existing biological knowledge [167]. Several databases exist that contain a variety of pathways based on prior biological knowledge. These include WikiPathways [167], Reactome [168], BioCarta [169] and KEGG [170]. Each of these databases combines knowledge from a different perspective. WikiPathways contains for instance disease specific pathways, whereas KEGG pathways generally represent metabolic processes in different species. The pathways in these databases allow users to project their data and perform statistics to determine which pathways, instead of single genes, play a role in a disease process.

\subsubsection{Metabolomics}

The metabolome represents the collection of all metabolites in a cell that are produced by the overall metabolism of an organism [171]. In contrast to transcriptomics, metabolomics provides the link between genotype and phenotype as it directly measures alterations in the cellular metabolism that result in phenotypical changes [171]. Metabolomics analysis can be done in a targeted manner, for which prior knowledge is utilized to focus on a subset of metabolites, or in a non-targeted way, where the whole metabolome is measured $[172,173]$. The most common techniques for measuring metabolomics are nuclear magnetic resonance (NMR) spectroscopy and MS [174].

\subsubsection{Omics Integration}

As each omics technology provides only one perspective of disease, different omics datasets should be combined to investigate the different aspects of a disease, identify novel relationships or gain new insights. Different statistical methods exist to integrate omics datasets for integrated analysis [175].

- Correlation-based integration. This method uses univariate Pearson or Spearman correlation analysis to calculate correlations between the variables from different omics datasets. However, some challenges occur using this approach since gene expression responds at a different pace than metabolites, even if measured at the same time. Significant correlations therefore need to be interpreted with caution.

- Concatenation-based integration. This can be achieved in different ways. First, Canonical Correlation Analysis (CCA) can be used for data integration. This method is a feature-based approach that allows for dimension reduction and fusion of the maximally correlated subsets of each dataset, thus achieving multivariate correlation-based data fusion [176]. Second, different types of data fusion exist that can combine different datasets based on their characteristics. A comprehensive review of data fusion techniques can be found in [177]. Third, a priori biological knowledge can be used to achieve concatenation of different datasets. For instance, pathway databases are available online that contain information about genes and metabolites, which can be used to retrieve and combine relevant biological knowledge from each separate dataset [167]. However, 
for breathomics, pathway-based integration is not yet possible as the biological origin of most VOCs remains elusive and cannot be found in a pathway database.

- Algorithm-based integration. Several methods exist to achieve this type of integration. They work in one of three ways. (i) One model is created for each omics dataset, which are then combined at a later stage. (ii) One omics dataset is used to predict the other and vice versa through for instance orthogonal partial least squares discriminant analysis (OPLS-DA). (iii) The third multivariate integration method is called Canonical Correlation Analysis (CCA), which is an extension of the correlation-based integration method described above. Groups of variables are correlated to one another instead of individual variables.

\subsection{The aims of this thesis}

The overall aim of the present thesis is to examine the several aspects of the use of VOCs profiling by GC-MS in lung associated diseases. To achieve this, the thesis encompasses 7 studies, of which each addresses one of the following aims: (i) to review the current data pre-processing and analysis strategies for exhaled breath research, (ii) to investigate the potential of exhaled breath for the diagnosis of selected lung diseases, (iii) to test the validity of discriminatory VOCs using independent external validation, (iv) to examine the use of VOCs in the in vitro headspace of cells and bacteria and (v) to identify plausible endogenous sources of VOCs excreted in vitro.

The first aim of the thesis was accomplished in chapter 2, where the current knowledge concerning data preprocessing and analysis was gathered to provide researchers with a strategy to analyze exhaled breath data.

The second aim of this thesis was tested in chapters $\mathbf{3}$ and $\mathbf{4}$, which aimed to investigate the value of exhaled breath analysis for the diagnosis of VAP and ILD. The aim of chapter 3 was to investigated whether exhaled breath could be used to diagnose patients with VAP, a nosocomial infection occurring in the intensive care unit (ICU) [178], with an attributable mortality risk of 13\% [179]. Bronchoalveolar lavage and subsequent microbial analysis is the current diagnostic golden standard, yet is invasive and time-consuming [180].

In chapter 4, exhaled breath analysis was used to distinguish between iodiopathic pulmonary fibrosis (IPF) and connective tissue disease-associated interstitial lung disease (CTD-ILD) patients. CTD-ILD is a heterogeneous group of diverse CTDs that have caused pulmonary complications with ILD as a result [181]. IPF is characterized by irreversible scarring of the lung tissue and has a poor prognosis and survival rates [182] These two ILD subtypes are difficult to distinguish from one another using the current clinical standards, therefore the aim was to identify a group of VOCs that could aid in this differentiation between these similar, yet different diseases.

The third aim of the thesis was tested in chapter $\mathbf{5}$ using sarcoidosis as a model disease. Sarcoidosis is a multisystem granulomatous disease with a great variety in the clinical phenotype [183] presenting with symptoms such as extreme fatigue, coughing, wheezing and dyspnea $[184,185]$. To evaluate the third aim of this thesis, exhaled breath samples of sarcoidosis patients and healthy controls were collected at 
two different hospitals. The data from one hospital was used to identify VOCs that could discriminate the patients from controls, whereas the data collected at the other hospital was used as an independent external validation of the identified VOCs.

The fourth aim of the thesis was tested in chapters 6 and 7. These studies were carried out in vitro and aimed to assess the value of VOCs in vitro. Chapter 6 investigated whether five different bacterial strains could be discriminated using VOCs excreted in the in vitro headspace, whereas chapter 7 used a well-understood chemical exposure to identify VOCs that were excreted as a result of that exposure.

The fifth and final aim was tested in chapter 8, which integrated breathomics, transcriptomics and metabolomics data collected after in vitro exposure of the BEAS2B lung epithelial cell line to a carcinogen. This study examined the relationships between VOCs, metabolites and pathways to elucidate the endogenous sources of VOCs. 


\section{Bibliography}

[1] Meggie Hakim, Yoav Y. Broza, Orna Barash, et al. Volatile organic compounds of lung cancer and possible biochemical pathways. Chemical Reviews, 112(11):5949-5966, nov 2012.

[2] a W Boots, A Smolinska, J.J.B.N. van Berkel, et al. Identification of microorganisms based on gas chromatography-mass spectrometric analysis of volatile organic compounds in headspace gases. Journal of biomedicine E3 biotechnology, 8(2):1752, jun 2014.

[3] Fraser Lough, John D. Perry, Stephen P. Stanforth, and John R. Dean. Detection of exogenous VOCs as a novel invitro diagnostic technique for the detection of pathogenic bacteria. $\operatorname{Tr} A C$ Trends in Analytical Chemistry, 87:71-81, 2017.

[4] Kseniya Dryahina, Kristýna Sovová, Alexandr Nemec, and Patrik Španěl. Differentiation of pulmonary bacterial pathogens in cystic fibrosis by volatile metabolites emitted by their in vitro cultures: Pseudomonas aeruginosa, Staphylococcus aureus, Stenotrophomonas maltophilia and the Burkholder. Journal of Breath Research, 10(3):037102, aug 2016.

[5] K D Nizio, K A Perrault, A N Troobnikoff, et al. $<\mathrm{i}>$ In vitro $</ \mathrm{i}>$ volatile organic compound profiling using GCGC-TOFMS to differentiate bacteria associated with lung infections: a proof-of-concept study. Journal of Breath Research, 10(2):026008, apr 2016.

[6] A H Neerincx, B P Geurts, M F J Habets, et al. Identification of <i>Pseudomonas aeruginosa $</ \mathrm{i}>$ and $<\mathrm{i}>$ Aspergillus fumigatus $</ \mathrm{i}>$ mono- and co-cultures based on volatile biomarker combinations. Journal of Breath Research, 10(1):016002, jan 2016.

[7] Lieuwe D. J. Bos, Peter J. Sterk, Marcus J. Schultz, L Hofmann, and S Ulbricht. Volatile Metabolites of Pathogens: A Systematic Review. PLoS Pathogens, 9(5):e1003311, may 2013.

[8] Marc Philippe Van Der Schee, Tamara Paff, Paul Brinkman, et al. Breathomics in lung disease. Chest, 147(1):224-231, jan 2015.

[9] A Tangerman, M T Meuwese-Arends, and J B Jansen. Cause and composition of foetor hepaticus. Lancet (London, England), 343(8895):483, feb 1994.

[10] M R Wills and J Savory. Biochemistry of renal failure. Annals of clinical and laboratory science, 11(4):292-9.

[11] L Laffel. Ketone bodies: a review of physiology, pathophysiology and application of monitoring to diabetes. Diabetes/metabolism research and reviews, 15(6):412-426, 1999.

[12] T C Vary, J H Siegel, T Nakatani, T Sato, and H Aoyama. A biochemical basis for depressed ketogenesis in sepsis. J Trauma, 26(5):419-425, 1986.

[13] Ronny Schnabel, Rianne Fijten, Agnieszka Smolinska, et al. Analysis of volatile organic compounds in exhaled breath to diagnose ventilator-associated pneumonia. Scientific Reports, 5(October):17179, jan 2015. 
[14] J. King, P. Mochalski, K. Unterkofler, et al. Breath isoprene: Muscle dystrophy patients support the concept of a pool of isoprene in the periphery of the human body. Biochemical and Biophysical Research Communications, 423(3):526-530, 2012.

[15] Julian King, a Kupferthaler, K Unterkofler, et al. Isoprene and acetone concentration profiles during exercise on an ergometer. Journal of breath research, 3(2):027006, jun 2009.

[16] T Karl, P Prazeller, D Mayr, et al. Human breath isoprene and its relation to blood cholesterol levels: new measurements and modeling. Journal of applied physiology (Bethesda, Md. : 1985), 91(2):762-70, aug 2001.

[17] Kim DG G van de Kant, Linda JTM T M van der Sande, Quirijn Jöbsis, Onno CP P van Schayck, and Edward Dompeling. Clinical use of exhaled volatile organic compounds in pulmonary diseases: a systematic review. Respiratory research, 13(1):117, dec 2012.

[18] L T McGrath, R Patrick, P Mallon, et al. Breath isoprene during acute respiratory exacerbation in cystic fibrosis. Eur Respir J, 16, 2000.

[19] J Scholpp, J K Schubert, W Miekisch, and K Geiger. Breath markers and soluble lipid peroxidation markers in critically ill patients. Clin Chem Lab Med, 40, 2002.

[20] J K Schubert, W P Muller, A Benzing, and K Geiger. Application of a new method for analysis of exhaled gas in critically ill patients. Intensive Care Med, 24, 1998.

[21] Matthias Lechner, Berthold Moser, David Niederseer, et al. Gender and age specific differences in exhaled isoprene levels. Respiratory Physiology $\&$ Neurobiology, 154(3):478-483, dec 2006.

[22] C M Kneepkens, Guy Lepage, Claude C. Roy, et al. The potential of the hydrocarbon breath test as a measure of lipid peroxidation. Free Radical Biology and Medicine, 17(2):127-60, aug 1994.

[23] D J Betteridge. What is oxidative stress? Metabolism: clinical and experimental, 49(2 Suppl 1):3-8, feb 2000 .

[24] J M Gutteridge. Lipid peroxidation and antioxidants as biomarkers of tissue damage. Clinical Chemistry, 41(12), 1995.

[25] Satori A Marchitti, Chad Brocker, Dimitrios Stagos, and Vasilis Vasiliou. Non-P450 aldehyde oxidizing enzymes: the aldehyde dehydrogenase superfamily. Expert opinion on drug metabolism \& toxicology, 4(6):697-720, jun 2008.

[26] H Esterbauer, R J Schaur, and H Zollner. Chemistry and biochemistry of 4-hydroxynonenal, malonaldehyde and related aldehydes. Free radical biology \& medicine, 11(1):81-128, 1991.

[27] Ankur Kansal. Sources and reactivity of NMHCs and VOCs in the atmosphere: A review. Journal of Hazardous Materials, 166(1):17-26, jul 2009.

[28] OA US EPA. Overview of Greenhouse Gases.

[29] Hanwant B. Singh and Patrick B. Zimmerman. Atmospheric distribution and sources of nonmethane hydrocarbons. jan 1992.

[30] Marcel Dicke and Francesco Loreto. Induced plant volatiles: from genes to climate change. Trends in plant science, 15(3):115-7, mar 2010.

[31] Wouter Kegge, Paulien Gankema, and Ronald Pierik. Plant-produced volatile organic compounds. Access Science, 2013.

[32] Joëlle K. Muhlemann, Antje Klempien, and Natalia Dudareva. Floral volatiles: from biosynthesis to function. Plant, Cell ES Environment, 37(8):1936-1949, aug 2014.

[33] Dušan Materić, Dan Bruhn, Claire Turner, et al. Methods in Plant Foliar Volatile Organic Compounds Research. Applications in Plant Sciences, 3(12):1500044, dec 2015.

[34] Hagit P. Affek and Dan Yakir. Protection by Isoprene against Singlet Oxygen in Leaves. Plant Physiology, 129(1), 2002.

[35] J HOLOPAINEN. Multiple functions of inducible plant volatiles. Trends in Plant Science, 9(11):529-533, nov 2004. 
[36] R Koppmann, K von Czapiewski, and J S Reid. A review of biomass burning emissions, part I: gaseous emissions of carbon monoxide, methane, volatile organic compounds, and nitrogen containing compounds. Atmospheric Chemistry and Physics Discussions, 5(5):10455-10516, 2005.

[37] Robin Smit, Leonidas Ntziachristos, and Paul Boulter. Validation of road vehicle and traffic emission models A review and meta-analysis. Atmospheric Environment, 44(25):2943-2953, aug 2010.

[38] Faisal I Khan and Aloke Kr. Ghoshal. Removal of Volatile Organic Compounds from polluted air. Journal of Loss Prevention in the Process Industries, 13(6):527-545, nov 2000.

[39] Wolfram Miekisch, Jochen K Schubert, and Gabriele F.E Noeldge-Schomburg. Diagnostic potential of breath analysisfocus on volatile organic compounds. Clinica Chimica Acta, 347(1):2539 , sep 2004.

[40] Agnes W Boots, Joep J B N van Berkel, Jan W Dallinga, et al. The versatile use of exhaled volatile organic compounds in human health and disease. Journal of Breath Research, 6(2):27108, jun 2012.

[41] Charlotte M. Robroeks, Joep J. Van Berkel, Quirijn Jöbsis, et al. Exhaled volatile organic compounds predict exacerbations of childhood asthma in a 1-year prospective study. European Respiratory Journal, 42(1):98-106, 2013.

[42] John B. (John Burnard) West and Andrew Luks. West's respiratory physiology : the essentials.

[43] L E Gustafsson, A M Leone, M G Persson, N P Wiklund, and S Moncada. Endogenous nitric oxide is present in the exhaled air of rabbits, guinea pigs and humans. Biochemical and biophysical research communications, 181(2):852-7, dec 1991.

[44] Wolfram Miekisch, Sabine Kischkel, Annika Sawacki, et al. Impact of sampling procedures on the results of breath analysis. Journal of Breath Research, 2(2):026007, jun 2008.

[45] N. Fens, M. P. van der Schee, P. Brinkman, and P. J. Sterk. Exhaled breath analysis by electronic nose in airways disease. Established issues and key questions. Clinical Es Experimental Allergy, 43(7):705-715, jul 2013.

[46] Alphus D. Wilson and Manuela Baietto. Advances in electronic-nose technologies developed for biomedical applications. Sensors, 11(1):1105-1176, 2011.

[47] Helko Borsdorf and Gary A. Eiceman. Ion Mobility Spectrometry: Principles and Applications. Applied Spectroscopy Reviews, 41(4):323-375, aug 2006.

[48] Jonathan Beauchamp. Current sampling and analysis techniques in breath researchresults of a task force poll. Journal of Breath Research, 9(4):047107, nov 2015.

[49] Célia Lourenço and Claire Turner. Breath analysis in disease diagnosis: methodological considerations and applications. Metabolites, 4(2):465-98, jun 2014.

[50] Sabine Kischkel, Wolfram Miekisch, Patricia Fuchs, and Jochen K. Schubert. Breath analysis during one-lung ventilation in cancer patients. European Respiratory Journal, 40(3):706-713, sep 2012 .

[51] Inbar Nardi-Agmon, Manal Abud-Hawa, Ori Liran, et al. Exhaled Breath Analysis for Monitoring Response to Treatment in Advanced Lung Cancer. Journal of Thoracic Oncology, 11(6):827-837, jun 2016.

[52] Changsong Wang, Ran Dong, Xiaoyang Wang, et al. Exhaled volatile organic compounds as lung cancer biomarkers during one-lung ventilation. Scientific Reports, 4(1):7312, may 2015.

[53] Wojciech Filipiak, Anna Filipiak, Andreas Sponring, et al. Comparative analyses of volatile organic compounds (VOCs) from patients, tumors and transformed cell lines for the validation of lung cancer-derived breath markers. Journal of Breath Research, 8(2):22, may 2013.

[54] Dillys van Vliet, Agnieszka Smolinska, Quirijn Jöbsis, et al. Can exhaled volatile organic compounds predict asthma exacerbations in children? Journal of Breath Research, 11(1):016016, $\operatorname{mar} 2017$.

[55] P. Brinkman, M. A. van de Pol, M. G. Gerritsen, et al. Exhaled breath profiles in the monitoring of loss of control and clinical recovery in asthma. Clinical \& Experimental Allergy, jul 2017. 
[56] David Smith, Kristýna Sovová, Kseniya Dryahina, et al. Breath concentration of acetic acid vapour is elevated in patients with cystic fibrosis. Journal of Breath Research, 10(2):021002, may 2016.

[57] Patrik Španěl, Kristýna Sovová, Kseniya Dryahina, et al. Do linear logistic model analyses of volatile biomarkers in exhaled breath of cystic fibrosis patients reliably indicate $<\mathrm{i}>$ Pseudomonas aeruginosa $</ \mathrm{i}>$ infection? Journal of Breath Research, 10(3):036013, aug 2016.

[58] R. Kramer, A. Sauer-Heilborn, T. Welte, et al. A rapid method for breath analysis in cystic fibrosis patients. European Journal of Clinical Microbiology $\&$ Infectious Diseases, 34(4):745751, apr 2015.

[59] M Phillips, V Basa-Dalay, G Bothamley, et al. Breath biomarkers of active pulmonary tuberculosis. Tuberculosis, 90, 2010.

[60] S. J. Fowler, M. Basanta-Sanchez, Y. Xu, R. Goodacre, and P. M. Dark. Surveillance for lower airway pathogens in mechanically ventilated patients by metabolomic analysis of exhaled breath: a case-control study. Thorax, 70(4):320-325, apr 2015.

[61] Michael Phillips, Thomas L. Bauer, Renee N. Cataneo, et al. Blinded Validation of Breath Biomarkers of Lung Cancer, a Potential Ancillary to Chest CT Screening. PLOS ONE, 10(12):e0142484, dec 2015.

[62] Bernard (Bernard A.) Rosner. Fundamentals of biostatistics. Brooks/Cole, Cengage Learning, 2011.

[63] Daniel J. Denis. Applied univariate, bivariate, and multivariate statistics.

[64] William S Noble. How does multiple testing correction work? Nature Biotechnology, 27(12):1135-1137, dec 2009.

[65] Richard G. Brereton and Gavin R. Lloyd. Partial least squares discriminant analysis: taking the magic away. Journal of Chemometrics, 28(4):213-225, apr 2014.

[66] Leo Breiman. Random forests. Mach. Learn., 45(1):5-32, oct 2001.

[67] Barbara G. Tabachnick and Linda S. Fidell. Using multivariate statistics. Pearson Education, 2013.

[68] M Phillips, K Gleeson, J M Hughes, et al. Volatile organic compounds in breath as markers of lung cancer: a cross-sectional study. Lancet, 353, 1999.

[69] A Gaida, O Holz, C Nell, et al. A dual center study to compare breath volatile organic compounds from smokers and non-smokers with and without COPD. Journal of Breath Research, 10(2):026006, apr 2016.

[70] Maria Basanta, Baharudin Ibrahim, Rachel Dockry, et al. Exhaled volatile organic compounds for phenotyping chronic obstructive pulmonary disease: a cross-sectional study. Respiratory research, 13(1):72, aug 2012.

[71] Charlotte M H H T Robroeks, Joep J B N van Berkel, Jan W. Dallinga, et al. Metabolomics of volatile organic compounds in cystic fibrosis patients and controls. Pediatric research, 68(1):75-80, jul 2010.

[72] Yishan Wang, Yanjie Hu, Di Wang, et al. The analysis of volatile organic compounds biomarkers for lung cancer in exhaled breath, tissues and cell lines. Cancer Biomarkers, 11(4):129-137, oct 2012 .

[73] Yuichi Sakumura, Yutaro Koyama, Hiroaki Tokutake, et al. Diagnosis by Volatile Organic Compounds in Exhaled Breath from Lung Cancer Patients Using Support Vector Machine Algorithm. Sensors (Basel, Switzerland), 17(2), feb 2017.

[74] Rosamaria Capuano, Marco Santonico, Giorgio Pennazza, et al. The lung cancer breath signature: a comparative analysis of exhaled breath and air sampled from inside the lungs. Scientific Reports, 5(1):16491, dec 2015.

[75] M Westhoff, P Litterst, L Freitag, et al. Ion mobility spectrometry for the detection of volatile organic compounds in exhaled breath of patients with lung cancer: results of a pilot study. Thorax, 64, 2009. 
[76] M Phillips, N Altorki, J H Austin, et al. Prediction of lung cancer using volatile biomarkers in breath. Cancer Biomark, 3, 2007.

[77] M Phillips, R N Cataneo, A R Cummin, et al. Detection of lung cancer with volatile markers in the breath. Chest, 123, 2003.

[78] Nir Peled, Meggie Hakim, Paul A Bunn, et al. Non-invasive breath analysis of pulmonary nodules. Journal of thoracic oncology : official publication of the International Association for the Study of Lung Cancer, 7(10):1528-33, oct 2012.

[79] A.-C. Hauschild, J.I. I Baumbach, and J.I. I Baumbach. Integrated statistical learning of metabolic ion mobility spectrometry profiles for pulmonary disease identification. Genetics and Molecular Research, 11(3):2733, aug 2012.

[80] J. W. Dallinga, C. M H H T Robroeks, J. J B N Van Berkel, et al. Volatile organic compounds in exhaled breath as a diagnostic tool for asthma in children. Clinical and Experimental Allergy, 40(1):68-76, jan 2010.

[81] Christopher Phillips, Neil Mac Parthaláin, Yasir Syed, et al. Short-Term Intra-Subject Variation in Exhaled Volatile Organic Compounds (VOCs) in COPD Patients and Healthy Controls and Its Effect on Disease Classification. Metabolites, 4(2):300-18, may 2014.

[82] M Phillips, V Basa-Dalay, J Blais, et al. Point-of-care breath test for biomarkers of active pulmonary tuberculosis. Tuberculosis, 92, 2012.

[83] M. Caldeira, R. Perestrelo, A.S. S Barros, et al. Allergic asthma exhaled breath metabolome: A challenge for comprehensive two-dimensional gas chromatography. J Chromatogr A, 1254:8797, sep 2012.

[84] Paola Gramatica. Principles of QSAR models validation: internal and external. QSAR ES Combinatorial Science, 26(5):694-701, may 2007.

[85] Agnieszka Smolinska, Ester M. M. EM Klaassen, JW Jan W. Dallinga, et al. Profiling of volatile organic compounds in exhaled breath as a strategy to find early predictive signatures of asthma in children. PLoS ONE, 9(4):e95668, apr 2014.

[86] Vasiliki Besa, Helmut Teschler, Isabella Kurth, et al. Exhaled volatile organic compounds discriminate patients with chronic obstructive pulmonary disease from healthy subjects. International journal of chronic obstructive pulmonary disease, 10:399-406, 2015.

[87] A. H J Kolk, J. J B N Van Berkel, M. M. Claassens, et al. Breath analysis as a potential diagnostic tool for tuberculosis. International Journal of Tuberculosis and Lung Disease, 16(6):777-782, 2012.

[88] Meijun Li and Geoffrey S. Ellis. Qualitative and Quantitative Analysis of Dibenzofuran, Alkyldibenzofurans, and Benzo $[<\mathrm{i}>\mathrm{b}</ \mathrm{i}\rangle]$ naphthofurans in Crude Oils and Source Rock Extracts. Energy \& Fuels, 29(3):1421-1430, mar 2015.

[89] A Wehinger, A Schmid, S Mechtcheriakov, et al. Lung cancer detection by proton transfer reaction mass-spectrometric analysis of human breath gas. Int J Mass Spectrom, 265, 2007.

[90] Ester M. M. Klaassen, Kim D. G. van de Kant, Quirijn Jöbsis, et al. Exhaled Biomarkers and Gene Expression at Preschool Age Improve Asthma Prediction at 6 Years of Age. American Journal of Respiratory and Critical Care Medicine, 191(2):201-207, jan 2015.

[91] M M Steeghs, S M Cristescu, P Munnik, P Zanen, and F J Harren. An off-line breath sampling and analysis method suitable for large screening studies. Physiol Meas, 28, 2007.

[92] Gary S. Collins, Johannes B. Reitsma, Douglas G. Altman, and Karel G.M. Moons. Transparent Reporting of a multivariable prediction model for Individual Prognosis Or Diagnosis (TRIPOD): The TRIPOD Statement. Annals of Internal Medicine, 162(1):55, jan 2015.

[93] J. J B N Van Berkel, J. W. Dallinga, G. M. Möller, et al. A profile of volatile organic compounds in breath discriminates COPD patients from controls. Respiratory Medicine, 104(4):557-563, apr 2010 .

[94] Yingchang Zou, Xi Zhang, Xing Chen, et al. Optimization of volatile markers of lung cancer to exclude interferences of non-malignant disease. Cancer Biomarkers, 14(5):371-379, jan 2014. 
[95] L Callol-Sanchez, M A Munoz-Lucas, O Gomez-Martin, et al. Observation of nonanoic acid and aldehydes in exhaled breath of patients with lung cancer. Journal of Breath Research, 11(2):026004, apr 2017.

[96] Tsuyoshi Oguma, Takashi Nagaoka, Muneshige Kurahashi, et al. Clinical contributions of exhaled volatile organic compounds in the diagnosis of lung cancer. PloS one, 12(4):e0174802, 2017.

[97] Tali Feinberg, Layah Alkoby-Meshulam, Jens Herbig, et al. Cancerous glucose metabolism in lung cancerevidence from exhaled breath analysis. Journal of Breath Research, 10(2):026012, jun 2016 .

[98] Erin M. Schumer, Matthew C. Black, Michael Bousamra, et al. Normalization of Exhaled Carbonyl Compounds After Lung Cancer Resection. The Annals of Thoracic Surgery, 102(4):1095-1100, oct 2016.

[99] Tomasz Ligor, Łukasz Pater, and Bogusław Buszewski. Application of an artificial neural network model for selection of potential lung cancer biomarkers. Journal of Breath Research, 9(2):027106, may 2015.

[100] Wen Ma, Peng Gao, Jun Fan, Yuki Hashi, and Zilin Chen. Determination of breath gas composition of lung cancer patients using gas chromatography/mass spectrometry with monolithic material sorptive extraction. Biomedical Chromatography, 29(6):961-965, jun 2015.

[101] Erin M. Schumer, Jaimin R. Trivedi, Victor van Berkel, et al. High sensitivity for lung cancer detection using analysis of exhaled carbonyl compounds. The Journal of Thoracic and Cardiovascular Surgery, 150(6):1517-1524, dec 2015.

[102] Michael Bousamra, Erin Schumer, Mingxiao Li, et al. Quantitative analysis of exhaled carbonyl compounds distinguishes benign from malignant pulmonary disease. The Journal of Thoracic and Cardiovascular Surgery, 148(3):1074-1081, sep 2014.

[103] Xiao-An Fu, Mingxiao Li, Ralph J. Knipp, Michael H. Nantz, and Michael Bousamra. Noninvasive detection of lung cancer using exhaled breath. Cancer Medicine, 3(1):174-181, feb 2014.

[104] Hiroshi Handa, Ayano Usuba, Sasidhar Maddula, et al. Exhaled Breath Analysis for Lung Cancer Detection Using Ion Mobility Spectrometry. PLoS ONE, 9(12):e114555, dec 2014.

[105] B Buszewski, T Ligor, T Jezierski, et al. Identification of volatile lung cancer markers by gas chromatography-mass spectrometry: comparison with discrimination by canines. Anal Bioanal Chem, 404, 2012.

[106] J Rudnicka, T Kowalkowski, T Ligor, and B Buszewski. Determination of volatile organic compounds as biomarkers of lung cancer by SPME-GC-TOF/MS and chemometrics. J Chromatogr B Analyt Technol Biomed Life Sci, 879, 2011.

[107] A Ulanowska, T Kowalkowski, E Trawinska, and B Buszewski. The application of statistical methods using VOCs to identify patients with lung cancer. J Breath Res, 5, 2011.

[108] P Fuchs, C Loeseken, J K Schubert, and W Miekisch. Breath gas aldehydes as biomarkers of lung cancer. Int J Cancer, 126, 2010.

[109] S Kischkel, W Miekisch, A Sawacki, et al. Breath biomarkers for lung cancer detection and assessment of smoking related effects-confounding variables, influence of normalization and statistical algorithms. Clin Chim Acta, 411, 2010.

[110] G Peng, M Hakim, Y Y Broza, et al. Detection of lung, breast, colorectal, and prostate cancers from exhaled breath using a single array of nanosensors. BJC, 103, 2010.

[111] G Song, T Qin, H Liu, et al. Quantitative breath analysis of volatile organic compounds of lung cancer patients. Lung Cancer, 67, 2010.

[112] Amel Bajtarevic, Clemens Ager, Martin Pienz, et al. Noninvasive detection of lung cancer by analysis of exhaled breath. BMC cancer, 9(1):348, 2009.

[113] M Ligor, T Ligor, A Bajtarevic, et al. Determination of volatile organic compounds in exhaled breath of patients with lung cancer using solid phase microextraction and gas chromatography mass spectrometry. Clin Chem Lab Med, 47, 2009. 
[114] M Crohns, S Saarelainen, J Laitinen, et al. Exhaled pentane as a possible marker for survival and lipid peroxidation during radiotherapy for lung cancer-a pilot study. Free Radic Res, 43, 2009.

[115] E M Gaspar, A F Lucena, J da Costa, and H das Neves. Organic metabolites in exhaled human breath-a multivariate approach for identification of biomarkers in lung disorders. $J$ Chromatogr A, 1216, 2009.

[116] G Peng, U Tisch, O Adams, et al. Diagnosing lung cancer in exhaled breath using gold nanoparticles. Nat Nanotechnol, 4, 2009.

[117] K D Skeldon, L C McMillan, C A Wyse, et al. Application of laser spectroscopy for measurement of exhaled ethane in patients with lung cancer. Respir Med, 100, 2006.

[118] Diana Poli, Paolo Carbognani, Massimo Corradi, et al. Exhaled volatile organic compounds in patients with non-small cell lung cancer: cross sectional and nested short-term follow-up study. Respir Res, 6(1):71, dec 2005.

[119] G Preti, J N Labows, J G Kostelc, S Aldinger, and R Daniele. Analysis of lung air from patients with bronchogenic carcinoma and controls using gas chromatography-mass spectrometry. J Chromatogr, 432, 1988.

[120] S M Gordon, J P Szidon, B K Krotoszynski, R D Gibbons, and H J O'Neill. Volatile organic compounds in exhaled air from patients with lung cancer. Clin Chem, 31, 1985.

[121] E van Mastrigt, A Reyes-Reyes, K Brand, et al. Exhaled breath profiling using broadband quantum cascade laser-based spectroscopy in healthy children and children with asthma and cystic fibrosis. Journal of Breath Research, 10(2):026003, apr 2016.

[122] Norbert Meyer, Jan W Dallinga, Sarah Janine Nuss, et al. Defining adult asthma endotypes by clinical features and patterns of volatile organic compounds in exhaled air. Respiratory research, 15(1):136, nov 2014.

[123] Florian Gahleitner, Cristina Guallar-Hoyas, Caroline S Beardsmore, Hitesh C Pandya, and CL Paul Thomas. Metabolomics pilot study to identify volatile organic compound markers of childhood asthma in exhaled breath. Bioanalysis, 5(18):2239-2247, sep 2013.

[124] P Paredi, S A Kharitonov, and P J Barnes. Elevation of exhaled ethane concentration in asthma. Am J Respir Crit Care Med, 162, 2000.

[125] José Javier Jareño-Esteban, M. Ángeles Muñoz-Lucas, Óscar Gómez-Martín, et al. Study of 5 Volatile Organic Compounds in Exhaled Breath in Chronic Obstructive Pulmonary Disease. Archivos de Bronconeumología (English Edition), 53(5):251-256, may 2017.

[126] M Allers, J Langejuergen, A Gaida, et al. Measurement of exhaled volatile organic compounds from patients with chronic obstructive pulmonary disease (COPD) using closed gas loop GCIMS and GC-APCI-MS. Journal of Breath Research, 10(2):026004, apr 2016.

[127] P Paredi, S A Kharitonov, D Leak, et al. Exhaled ethane, a marker of lipid peroxidation, is elevated in chronic obstructive pulmonary disease. Am J Respir Crit Care Med, 162, 2000.

[128] B Enderby, D Smith, W Carroll, and W Lenney. Hydrogen cyanide as a biomarker for Pseudomonas aeruginosa in the breath of children with cystic fibrosis. Pediatr Pulmonol, 44, 2009.

[129] M. Barker, M Hengst, J Schmid, et al. Volatile organic compounds in the exhaled breath of young patients with cystic fibrosis. European Respiratory Journal, 27(5):929-36, feb 2006.

[130] M. A. Kamboures, D. R. Blake, D. M. Cooper, et al. Breath sulfides and pulmonary function in cystic fibrosis. Proceedings of the National Academy of Sciences, 102(44):15762-15767, nov 2005.

[131] Paolo Paredi, Sergei A. Kharitonov, David Leak, et al. Exhaled Ethane Is Elevated in Cystic Fibrosis and Correlates with Carbon Monoxide Levels and Airway Obstruction. American Journal of Respiratory and Critical Care Medicine, 161(4):1247-1251, apr 2000.

[132] M Syhre, L Manning, S Phuanukoonnon, P Harino, and S T Chambers. The scent of Mycobacterium tuberculosis-part II breath. Tuberculosis, 89, 2009.

[133] Yu-ichi Yamada, Gen Yamada, Mitsuo Otsuka, et al. Volatile Organic Compounds in Exhaled Breath of Idiopathic Pulmonary Fibrosis for Discrimination from Healthy Subjects. Lung, 195(2):247-254, apr 2017. 
[134] M Westhoff, P Litterst, L Freitag, and J I Baumbach. Ion mobility spectrometry in the diagnosis of sarcoidosis: results of a feasibility study. Journal of physiology and pharmacology : an official journal of the Polish Physiological Society, 58 Suppl 5(Pt 2):739-51, nov 2007.

[135] S Kanoh, H Kobayashi, and K Motoyoshi. Exhaled ethane: an in vivo biomarker of lipid peroxidation in interstitial lung diseases. Chest, 128, 2005.

[136] L Blanchet, A Smolinska, A Baranska, et al. Factors that influence the volatile organic compound content in human breath. J Breath Res, 11(1):16013, feb 2017.

[137] Shamitha Dissanayake, Patty Lathan, and Todd Mlsna. Novel extraction of volatile biomarkers from canine breath for gas chromatographymass spectrometry. Journal of Breath Research, 6(4):041001, dec 2012.

[138] C A Wyse, S Love, R M Christley, et al. Validation of a method for collection and assay of pentane in the exhaled breath of the horse. Research in veterinary science, 76(2):109-12, apr 2004.

[139] Alexander A. Aksenov, Laura Yeates, Alberto Pasamontes, et al. Metabolite Content Profiling of Bottlenose Dolphin Exhaled Breath. Analytical Chemistry, 86(21):10616-10624, nov 2014.

[140] Konstantin O Zamuruyev, Alexander A Aksenov, Mark Baird, et al. Enhanced non-invasive respiratory sampling from bottlenose dolphins for breath metabolomics measurements. Journal of Breath Research, 10(4):046005, sep 2016.

[141] Raquel Cumeras, William Cheung, Frances Gulland, Dawn Goley, and Cristina Davis. Chemical Analysis of Whale Breath Volatiles: A Case Study for Non-Invasive Field Health Diagnostics of Marine Mammals. Metabolites, 4(3):790-806, sep 2014.

[142] Fangfang Liu, Wenli Li, Jürgen Pauluhn, Hubert Trübel, and Chen Wang. Rat models of acute lung injury: Exhaled nitric oxide as a sensitive, noninvasive real-time biomarker of prognosis and efficacy of intervention. Toxicology, 310:104-114, aug 2013.

[143] Sina Fischer, Phillip Trefz, Andreas Bergmann, et al. Physiological variability in volatile organic compounds (VOCs) in exhaled breath and released from faeces due to nutrition and somatic growth in a standardized caprine animal model. Journal of breath research, 9(2):027108, jun 2015.

[144] Stephanie Neuhaus, Luzia Seifert, Wolfgang Vautz, et al. Comparison of metabolites in exhaled breath and bronchoalveolar lavage fluid samples in a mouse model of asthma. Journal of Applied Physiology, 111(4):1088-95, oct 2011.

[145] Lieuwe D. J. Bos, Inez C. van Walree, Arend H. J. Kolk, et al. Alterations in exhaled breath metabolite-mixtures in two rat models of lipopolysaccharide-induced lung injury. Journal of Applied Physiology, 115(10):1487-95, nov 2013.

[146] W. Vautz, J. Nolte, A. Bufe, J. I. Baumbach, and M. Peters. Analyses of mouse breath with ion mobility spectrometry: a feasibility study. Journal of Applied Physiology, 108(3):697-704, mar 2010.

[147] C A Wyse, K Skeldon, J W Hotchkiss, et al. Effects of changes to the stable environment on the exhalation of ethane, carbon monoxide and hydrogen peroxide by horses with respiratory inflammation. The Veterinary record, 157(14):408-12, oct 2005.

[148] S Ghimenti, F Di Francesco, M Onor, et al. Post-operative elimination of sevoflurane anesthetic and hexafluoroisopropanol metabolite in exhaled breath: pharmacokinetic models for assessing liver function. Journal of Breath Research, 7(3):036001, jun 2013.

[149] Alan G. Barbour, Charlotte M. Hirsch, Arash Ghalyanchi Langeroudi, et al. Elevated Carbon Monoxide in the Exhaled Breath of Mice during a Systemic Bacterial Infection. PLoS ONE, 8(7):e69802, jan 2013.

[150] Damien Van Berlo, Catrin Albrecht, Ad M. Knaapen, et al. Comparative evaluation of the effects of short-term inhalation exposure to diesel engine exhaust on rat lung and brain. Archives of toxicology, 84(7):553-562, jul 2010.

[151] Wojciech Filipiak, Andreas Sponring, Tomas Mikoviny, et al. Release of volatile organic compounds (VOCs) from the lung cancer cell line CALU-1 in vitro. Cancer Cell International, $8(1): 17$, nov 2008. 
[152] Paweł Mochalski, Markus Theurl, Andreas Sponring, et al. Analysis of Volatile Organic Compounds Liberated and Metabolised by Human Umbilical Vein Endothelial Cells (HUVEC) In Vitro. Cell Biochemistry and Biophysics, 71(1):323-329, jan 2015.

[153] A Baranska, A Smolinska, A W Boots, J W Dallinga, and F J van Schooten. Dynamic collection and analysis of volatile organic compounds from the headspace of cell cultures. Journal of Breath Research, 9(4):047102, oct 2015.

[154] Paweł Mochalski, Andreas Sponring, Julian King, et al. Release and uptake of volatile organic compounds by human hepatocellular carcinoma cells (HepG2) in vitro. Cancer Cell International, 13(1):72, jul 2013.

[155] Andreas Sponring, Wojciech Filipiak, Tomas Mikoviny, et al. Release of volatile organic compounds from the lung cancer cell line NCI-H2087 in vitro. Anticancer research, 29(1):419-26, jan 2009 .

[156] Paweł Mochalski, Ramona Al-Zoairy, Andreas Niederwanger, Karl Unterkofler, and Anton Amann. Quantitative analysis of volatile organic compounds released and consumed by rat L6 skeletal muscle cells <i>in vitro</i>. Journal of Breath Research, 8(4):046003, oct 2014.

[157] Luca Lavra, Alexandro Catini, Alessandra Ulivieri, et al. Investigation of VOCs associated with different characteristics of breast cancer cells. Scientific Reports, 5(1):13246, oct 2015.

[158] Hossam Haick, Lu Amal, Bin-bin Ding, et al. The scent fingerprint of hepatocarcinoma: invitro metastasis prediction with volatile organic compounds (VOCs). International Journal of Nanomedicine, 7:4135, jul 2012.

[159] Kristin Schallschmidt, Roland Becker, Christian Jung, et al. Investigation of cell culture volatilomes using solid phase micro extraction: Options and pitfalls exemplified with adenocarcinoma cell lines. Journal of Chromatography B, 1006:158-166, dec 2015.

[160] Tali Feinberg, Jens Herbig, Ingrid Kohl, et al. Cancer metabolism: the volatile signature of glycolysis $<\mathrm{i}>$ in vitro $</ \mathrm{i}>$ model in lung cancer cells. Journal of Breath Research, 11(1):016008, jan 2017.

[161] Florence N Schleich, Jan W Dallinga, Monique Henket, et al. Volatile organic compounds discriminate between eosinophilic and neutrophilic inflammation in vitro. Journal of Breath Research, 10(1):016006, feb 2016.

[162] Nir Peled, Orna Barash, Ulrike Tisch, et al. Volatile fingerprints of cancer specific genetic mutations. Nanomedicine: Nanotechnology, Biology, and Medicine, 9(6):758-766, aug 2013.

[163] Jae Sung Pyo, Hyun Kyoung Ju, Jeong Hill Park, and Sung Won Kwon. Determination of volatile biomarkers for apoptosis and necrosis by solid-phase microextraction-gas chromatography/mass spectrometry: A pharmacometabolomic approach to cisplatin's cytotoxicity to human lung cancer cell lines. Journal of Chromatography B, 876(2):170-174, dec 2008.

[164] Casey E Romanoski, Christopher K Glass, Hendrik G Stunnenberg, Laurence Wilson, and Genevieve Almouzni. Epigenomics: Roadmap for regulation. Nature, 518(7539):314-6, feb 2015.

[165] Paul R Graves and Timothy A J Haystead. Molecular biologist's guide to proteomics. Microbiology and molecular biology reviews : MMBR, 66(1):39-63; table of contents, mar 2002.

[166] Joaquin Dopazo. Genomics and transcriptomics in drug discovery. Drug Discovery Today, 19(2):126-132, feb 2014.

[167] Martina Kutmon, Anders Riutta, Nuno Nunes, et al. WikiPathways: Capturing the full diversity of pathway knowledge. Nucleic Acids Research, 44(D1):D488-D494, jan 2016.

[168] David Croft, Antonio Fabregat Mundo, Robin Haw, et al. The Reactome pathway knowledgebase. Nucleic Acids Research, 42(D1):D472-D477, jan 2014.

[169] Darryl Nishimura. BioCarta. Biotech Software \& Internet Report: The Computer Software Journal for Scient, 2(3):117-120, 2001.

[170] M Kanehisa and S Goto. KEGG: kyoto encyclopedia of genes and genomes. Nucleic acids research, 28(1):27-30, jan 2000. 
[171] Oliver Fiehn. Metabolomics-the link between genotypes and phenotypes. Plant molecular biology, 48(1-2):155-71, jan 2002.

[172] Lee D. Roberts, Amanda L. Souza, Robert E. Gerszten, and Clary B. Clish. Targeted Metabolomics. In Current Protocols in Molecular Biology, volume Chapter 30, pages Unit 30.2.1-24. John Wiley \& Sons, Inc., Hoboken, NJ, USA, apr 2012.

[173] Nawaporn Vinayavekhin and Alan Saghatelian. Untargeted Metabolomics. In Current Protocols in Molecular Biology, volume Chapter 30, pages Unit 30.1.1-24. John Wiley \& Sons, Inc., Hoboken, NJ, USA, apr 2010.

[174] Abdul-Hamid M. Emwas. The Strengths and Weaknesses of NMR Spectroscopy and Mass Spectrometry with Particular Focus on Metabolomics Research. In Metabonomics, chapter The Streng, pages 161-193. Springer New York, 2015.

[175] Rachel Cavill, Danyel Jennen, Jos Kleinjans, and Jacob Jan Briedé. Transcriptomic and metabolomic data integration. Briefings in Bioinformatics, 17(5):891-901, sep 2016.

[176] Nicolle M. Correa, Tülay Adali, Yi-Ou Li, and Vince D. Calhoun. Canonical Correlation Analysis for Data Fusion and Group Inferences: Examining applications of medical imaging data. IEEE signal processing magazine, 27(4):39, 2010.

[177] A Carracedo, L C Cantley, and P P Pandolfi. Cancer metabolism: fatty acid oxidation in the limelight. Nat.Rev.Cancer, 13(1474-1768 (Electronic)):227-232, apr 2013.

[178] Amédée Ego, Jean Charles Preiser, and Jean Louis Vincent. Impact of diagnostic criteria on the incidence of ventilator-associated pneumonia. Chest, 147(2):347-355, 2015.

[179] Wilhelmina G. Melsen, Maroeska M. Rovers, Rolf H H Groenwold, et al. Attributable mortality of ventilator-associated pneumonia: A meta-analysis of individual patient data from randomised prevention studies. The Lancet Infectious Diseases, 13(8):665-671, 2013.

[180] J Y Fagon, Jean Chastre, Michel Wolff, et al. Invasive and noninvasive strategies for management of suspected ventilator-associated pneumonia. A randomized trial. Annals of internal medicine, 132(8):621-30, 2000.

[181] Rekha Vij and Mary E Strek. Diagnosis and treatment of connective tissue disease-associated interstitial lung disease. Chest, 143(3):814-824, mar 2013.

[182] K M Antoniou, G A Margaritopoulos, S Tomassetti, et al. Interstitial lung disease. Eur Respir Rev, 23(131):40-54, 2014.

[183] Gary W. Hunninghake, Ulrich Costabel, Masayuki Ando, et al. Statement on sarcoidosis. American Journal of Respiratory and Critical Care Medicine, 160(2):736-755, aug 1999.

[184] Marjolein Drent, Elyse E. Lower, and Jolanda De Vries. Sarcoidosis-associated fatigue. European Respiratory Journal, 40(1):255-263, jul 2012.

[185] Marc A. Judson. The Clinical Features of Sarcoidosis: A Comprehensive Review. Clinical Reviews in Allergy and Immunology, 49(1):63-78, aug 2015. 
CHAPTER 2

Current Breathomics - a review on data pre-processing techniques and machine learning in metabolomics breath analysis

A Smolinska, Hauschild AC, R Fijten, J Dallinga, J Baumbach, F van Schooten J Breath Res. 2014 Jun;8(2):027105 


\subsection{Abstract}

We define breathomics as the metabolomics study of exhaled air. It is a strongly emerging metabolomics research field that mainly focuses on health-related volatile organic compounds (VOCs). Since the amount of these compounds varies with health status, breathomics holds great promise to deliver non-invasive diagnostic tools. Thus, the main aim of breathomics is to find patterns of VOCs related to abnormal (for instance inflammatory) metabolic processes occurring in the human body. Recently, analytical methods for measuring VOCs in exhaled air with high resolution and high throughput have been extensively developed. Yet, the application of machine learning methods for fingerprinting VOC profiles in the breathomics is still in its infancy. Therefore, in this paper, we describe the current state of the art in data preprocessing and multivariate analysis of breathomics data. We start with the detailed pre-processing pipelines for breathomics data obtained from gas-chromatography mass spectrometry and an ion-mobility spectrometer coupled to multi-capillary columns. The outcome of data pre-processing is a matrix containing the relative abundances of a set of VOCs for a group of patients under different conditions (e.g. disease stage, treatment). Independently of the utilized analytical method, the most important question, 'which VOCs are discriminatory?', remains the same. Answers can be given by several modern machine learning techniques (multivariate statistics) and, therefore, are the focus of this paper. We demonstrate the advantages as well the drawbacks of such techniques. We aim to help the community to understand how to profit from a particular method. In parallel, we hope to make the community aware of the existing data fusion methods, as yet unresearched in breathomics.

\subsection{Introduction}

Searching for disease-specific markers (so-called biomarker discovery) has become a significant area in biomedical research. The main aim of biomarker discovery is to develop tools that indicate when a healthy state becomes dysfunctional, ideally at the earliest possible stage of the disease progression. In the field of metabolomics, the molecular compositions of biofluids, cells and/or tissues are investigated to find markers indicative to abnormal status of the human body. Similarly, exhaled air is a biological medium that carries relevant medical information. Thus, investigating metabolites in exhaled air, referred to as breathomics, may bring valuable information about malfunctions occurring in the human body $[1,2]$. As with other types of biological samples, exhaled air can be used to find a set of markers (breath prints) characteristic of abnormal health status. The target compounds detectably in the breath are volatile organic compounds (VOCs). They originate from normal and deviant (for instance inflammatory) metabolic processes occurring in the body [3, 4]. The use of VOCs as markers of exposure or disease has dramatically increased in the last decade because of the tremendous progress of chemical analytical platforms and pattern-recognition techniques. With such equipment at hand, a non-invasive biomarker breath profiling method is within reach.

A single breath sample often contains thousands of VOCs; therefore, the amount 
of data produced in breathomics is numerically large and biologically complex. Moreover, with decreasing hardware costs and increasing resolution and throughput, we are currently observing an explosion of the data volume. Next to the endogenously formed and metabolized compounds, exhaled air also contains exogenous compounds derived from environmental components that are exhaled again or derived from viruses and bacteria. This means that breathomics data contain various sources of variance, i.e., information of interest and unimportant variance connected to biological variation or noise in the data. As a result, one of the major research challenges is to extract information related to, e.g., diseases and to neglect and/or diminish the irrelevant variance in the data.

An array of sophisticated machine learning methods can be used to find reliable disease predictors [5-8]. In the remainder of this paper, we refer to them as multivariate statistical analysis. In traditional biomedical research, however, the sole use of p-values to show the significance of the findings is widespread. The p-value is the probability of getting the observed value of the test statistic, or a value with greater evidence against the null hypothesis if the null hypothesis is true. Even though the definition of the p-value seems clear, the inferential meaning of the p-value is very often misconstrued. The p-value does not carry information about the magnitude of the differences. Therefore, a statistically significant outcome (e.g. p-value ¡0.05) does not say anything about the clinical relevance of the outcome. A non-significant p-value only indicates that the data are consistent with the null hypothesis. In other words, possibly no difference exists between the groups; however, it does not mean that the null effect is the most likely. Goodman [9] describes 12 p-value misconceptions and explains the consequence of improper understanding of its meaning.

Similarly, Malley et al [10] discuss the restrictions of the use of p-values in biomedical research. It is clearly outlined that the use of a p-value as a quality measure is limited. The resulting p-value specifies if the null hypothesis is rejected, or it cannot be rejected at the defined significance level. Again, this does not mean that the null hypothesis is true. The p-value is not a probability statement of the hypothesis being true. The p-value does not give relevant information on physiological processes and might be even completely uninformative biologically. Moreover, correcting over several hundred tests by, e.g., Bonferroni or false discovery rate, assumes that the separate p-values are independent and there is no relation between any two VOCs. However, data produced in breathomics are frequently correlated as (often unknown) cascades of metabolic pathways and biochemical reactions connect to the measured VOCs. The significant p-value does not give the probability that by repeating the experiment the same conclusion can be drawn. Consequently, Malley et al suggest utilizing more sophisticated techniques and focusing on predicting the class (i.e. disease) of a given sample (i.e., patient) rather than on something that is merely significant. Obviously, this is the case with machine learning techniques.

Here, we present a comprehensive description of existing breathomics data analysis methodology. With respect to machine learning, note that the aim of this paper is not only to give a broad description of the available methods but also to provide clues when to apply them. Many different methods exist and, unfortunately, there is no golden rule in machine learning. Thus, here we give some dedicated breathomics data analysis guidelines. The paper first briefly covers the description of two different analytical 
approaches widely used in breathomics, namely gas chromatography coupled with mass spectrometry (GC-MS) and an ion-mobility spectrometer combined with a multicapillary column (MCC-IMS). Afterwards, we give a description of typical data preprocessing pipelines for GC-MS and MCC-IMS data sets. We deal with the important, but still sometimes neglected, aspects of scaling, normalization and transformation. In the main part, we focus on machine learning techniques. Among the manifold variety of methods available in the field, we select the most widely applied and most promising ones. Such multivariate statistical techniques can be divided into two types: explorative (also known as unsupervised or descriptive) $[11,12]$ and supervised approaches [12-14]. The first approach aims to explore and discover trends and correlations in the data without any a priori assumptions. Supervised techniques use a priori knowledge (e.g. class information) and are mostly used to train a mathematical model to discriminate and predict a class (disease) based on a preferably small set of selected VOCs extracted during variable selection. In the final part, we cover the relatively new area of data fusion.

\subsection{Instrumentation}

\subsubsection{Gas Chromatography - Mass Spectrometry}

GC-MS [15] is currently the most commonly applied analytical technique to measure trace gases in complex mixtures such as exhaled air $[16,17]$. This method includes a GC part, which separates the different compounds in the mixture and an MS part, which allows for compound detection and identification based on their total mass spectrum. The separation of compounds in the GC is based on interactions of compounds with the mobile phase and the lining of the capillary column material. The ionization of compounds takes place after entering the mass spectrometer. Various detectors can be used as a mass spectrometer. The time-of-flight (tof) [18] spectrometer is the most widely applied. In tof-MS, the time necessary to travel in an electric field from the ionization source to a detector plate is measured. Since all ions have the same kinetic energy $\left(E=\frac{1}{2} m v^{2}\right)$, ions with different mass-to-charge ratio $(\mathrm{m} / \mathrm{z}$ values $)$ are separated in the flight tube into groups or packets based on their velocity. GC-tof-MS is sensitive and robust. It measures all the mass fingerprints of the ionized compounds, and it has a high level of reproducibility. [4, 15]. However, it is a time-consuming method and on-line measurements are not possible. GC-tof-MS delivers highly accurate chromatograms (called breathograms in case of exhaled air) [4, 17]. figure 2.1(a) shows an example of such a breathogram. The x-axis represents the retention time which is proportional to the substance's affinity for the stationary phase while the $\mathrm{y}$-axis corresponds to the relative abundance of the VOC, derived from the total ion current (TIC) measured in the mass spectrometer. Every $0.2 \mathrm{~s}$ the MS produces a full mass spectrum. 

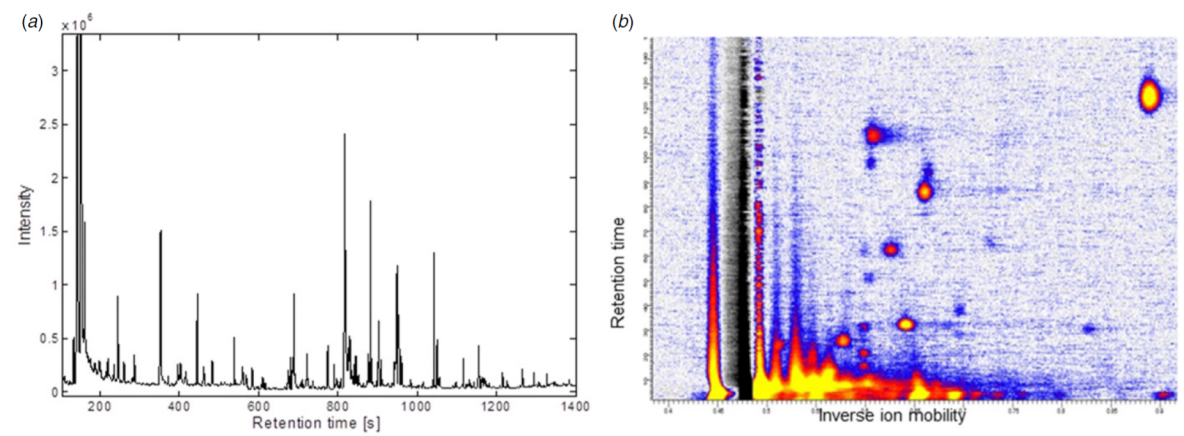

Figure 2.1: Examples of breathograms with both methods. (a) An example of a breathogram measured with GC-MS. The x-axis corresponds to the retention time which is proportional to the substance's affinity for the stationary phase, while the $\mathrm{y}$-axis reflects the relative abundance of the VOC, derived from the total ion current measured in the mass spectrometer. (b) An example of MCC-IMS breathograms. The $\mathrm{x}$-axis corresponds to the reduced inverse ion mobility $1 / \mathrm{K} 0(\mathrm{Vs} \mathrm{cm} 2)$ and it is proportional to the drift time (IMS), while the y-axis corresponds to the retention time (MCC). The colors reflect the signal height: yellow for the highest signal and white for the lowest.

\subsubsection{Ion-mobility spectrometer combined with a multi- capillary column}

An ion-mobility spectrometer combined with an MCC (MCC-IMS) is a well-known technology for detecting VOCs in air. The carrier gas drives the analytes into an MCC, where the pre-separation takes place [19]. After passing the column containing approximately 1000 parallel capillaries with an inner diameter of $40 \mu \mathrm{m}$, they reach the ionization chamber. Here, the analytes become chemically ionized by collisions with the reactant ions, which are carrier gas molecules previously ionized by a radioactive ionization source (usually ${ }^{63} \mathrm{Ni}$ ). The ion shutter opens cyclically and the resulting ions enter the drift region. Similarly to tof-MS, the ions gain energy from an external electric field and are guided toward a Faraday plate while a so-called drift gas flows in the opposite direction and prevents neutral molecules from entering the drift region. During their flight, the ionized molecules collide with the neutral drift gas molecules and are thereby separated by mass, shape and polarity. The signal recorded by the Faraday plate is called the ion-mobility spectrum. The MCC-IMS technique has a number of advantages in performance, cost and applicability. It has a low detection limit and measurements are inexpensive $(<5 \mathrm{EUR})$ and fast $(<5 \mathrm{~min})$, and it can handle the moisture that comes with exhaled air, which makes it suitable for many medical [19, 20] and biomedical [21] applications as well as for process analysis [22]. An example of an MCC-IMS breathogram is shown in figure 2.1(b). The y-axis corresponds to the retention time $(\mathrm{MCC})$ and the $\mathrm{x}$-axis to the reduced inverse ion mobility $1 / \mathrm{Ko}\left(\mathrm{Vs} \mathrm{cm}^{2}\right)$ which is proportional to the drift time (IMS) [23]. The signal height corresponds to the signal strength detected by the Faraday plate in the IMS 
device.

\subsection{Data pre-processing}

The output of analytical techniques such as GC-MS and MCC-IMS consists of the raw data. Proper pre-processing of the raw data is crucial for obtaining a reliable data matrix in which the actual statistical analysis can be performed. It is obvious that the data pre-processing is a fundamental enterprise since an unreliable data matrix will jeopardize the results at a later stage when applying the multivariate analysis (garbage in Gt garbage out). Moreover, proper data pre-processing greatly improves and simplifies the data analysis. Currently, many diverse methods and packages (commercially or freely available) exist for pre-processing of spectral data [24-28]. Data pre-processing can be divided on various sub-steps, such as denoising (smoothing), baseline correction, alignment across all samples, peak picking and combining of the peaks from numerous samples originating from the same compound. Finally, the peak areas matching different compounds are collected and converted into a data matrix for statistical analysis. As an example, we will discuss how data generated by GCMS and IMS have to be pre-processed in order to obtain more similar data that can subsequently be used for data analysis. Note, however, that similar pre-processing procedures are necessary for other analytical techniques as well, such as proton transfer MS (PTR-MS) and selected ion flow tube MS (SIFT-MS). A detailed description of pre-processing workflow for PTR-MS and SIFT-MS data can be found in [29-32]. In figure 2.2, a flowchart of the pre-processing of GC-MS data is shown.

\subsubsection{Data pre-processing of GC-MS data}

\subsubsection{Denoising and baseline correction}

The raw GC-MS data consist of two dimensions, i.e., $\mathrm{m} / \mathrm{z}$ value on the $\mathrm{x}$-axis and retention time on the y-axis. Therefore, for each measured breath sample, retention time and corresponding mass spectra are available. Retention time can be expressed as scan number since five scans are equal to $1 \mathrm{~s}$. In the mass spectrometer for each $\mathrm{m} / \mathrm{z}$ value, the intensity of the ion is recorded. By summing up the intensities of all peaks (i.e., $\mathrm{m} / \mathrm{z}$ values) in a mass spectrum, we obtain the TIC for one scan. This TIC represents the intensity at that scan in the chromatogram. The TIC is measured five times per second. All TICs together form the TIC chromatogram, which corresponds to the gas chromatogram. The gas chromatogram contains the scan number on the $\mathrm{x}$-axis and the intensity on the $\mathrm{y}$-axis.

The first step of pre-processing of the raw GC-MS data usually involves denoising and baseline correction. The main goal of denoising is to reduce the influence of random variations, i.e., noise introduced by variations in instrumental conditions. Several transformation functions such as wavelets and Fourier transformations can be applied to the original data, decomposing the spectra efficiently into noise and signal [33]. Trygg et al summarized several denoising techniques [23]. It is important to mention that transformation functions in wavelets are suitable for removing homoscedastic noise (i.e., Gaussian noise). However, usually signals produced by GC-MS contain 


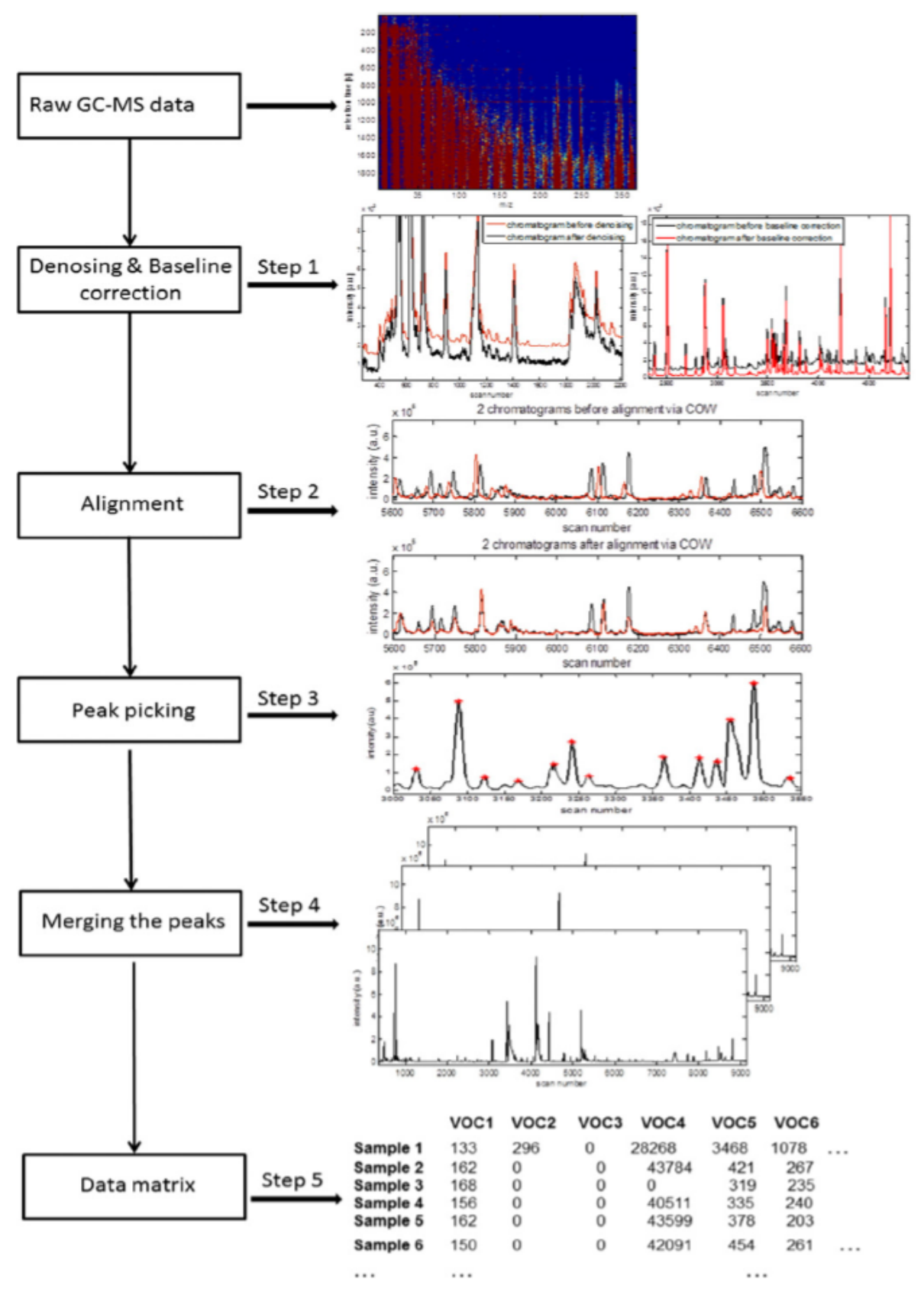

Figure 2.2: Example of a processing strategy of GC-MS data involving denoising and baseline correction (step 1), alignment of the retention times (step 2), pick picking (step 3), automated peak matching (based on the spectral signature; step 4) and construction of data matrix (step 5). 
heteroscedastic noise (i.e., proportional to the signal) as well. Thus, in order to reduce this noise, it is necessary to transform heteroscedastic noise to homoscedastic by e.g. logarithmic transformation of the data [34]. More details about this issue can be found elsewhere [35]. In figure 2.2 denoising of the chromatogram was performed via wavelets (with a Daubechies wavelet and two levels of compressions) [33].

The next step of data pre-processing is baseline (often called background) correction frequently done automatically using numerous types of polynomial fitting. Appropriate baseline removal is extremely important since poor baseline correction may lead to destruction of the data, affecting quantification and data analysis, but also the consecutive step, i.e., alignment. Nowadays different approaches are available, e.g., asymmetric least squares (ALS) [36] with B-splines, B-splines with penalization (i.e., P-splines) [37] or the use of an orthogonal basis of the background spectra [38]. In the flowchart shown in figure 2.2, the baseline correction was performed by means of $2 \mathrm{D}$ baseline estimation via ALS. Here, a least-squares approach with penalties is used to estimate the baseline. 2D ALS use B-splines, which are local basis functions, consisting of a number of polynomial pieces of low degree connected in a special way. Very significant parameters of the ALS approach are smoothing parameter, the order of differences d (i.e. polynomials of different degree) and weights p. For the last two parameters, second order of differences and p-value nearness to unity ensure good baseline estimation. In the case of smoothing parameter $\alpha$, the larger the value, the smoother (flatter) the estimated baseline becomes.

\subsubsection{Alignment}

The GC-MS produces gas chromatograms that may contain distortions of the retention time due to column ageing, changes in temperature or sometimes unknown deviations in instrumental conditions. Fluctuations in retention times across various measurements obscure statistical analysis and thus discovery of relevant patterns in the data. Therefore, accurate alignment of peaks is extremely important (step 2). It is important to point out that denoising and baseline correction are performed on the complete gas chromatogram while alignment and peak detection are performed on TIC. The problem of drifts in retention time of chromatographic data is thoroughly investigated, and thus new techniques to cope with this problem are being constantly developed.

Many methods and packages are commercially or freely available for spectral alignment, namely dynamic time warping (the oldest warping techniques), parametric time warping [39], correlation optimized warping (COW) [40], dynamic time warping [41], bidirectional best hits peak assignment [26], multiple alignment by bidirectional best hits peak assignment and cluster extension, center-star multiple alignment by pairwise partitioned dynamic time warping [26], MetAlign [24, 25], MZmine [42] and MetHR [43]. The most commonly used alignment techniques and methods are summarized by Jellema [44]. In step 2 of figure 2.2, the existing peak shifts between different chromatograms are removed by means of COW. This method warps (linearly stretches and compresses) one chromatogram to another by selecting input parameters such as section length (i.e., number of scans in one segment) and slack size (i.e., the maximum degree of warping allowed in one segment) that maximize the similarity 
between the two chromatograms using dynamic programming.

The chromatograms usually are warped to a reference chromatogram. There are several ways of selecting a reference chromatogram; for instance a median or mean of all measured chromatograms may be used, or the chromatogram with the highest overall correlation is chosen as the reference. At this point, the pre-processed GC-MS data are already ready for data analysis. However, it is common to continue with data pre-processing by performing peak picking. Commercially available software allows automatic detection of peaks in the complex GC-MS spectra.

\subsubsection{Peak picking and data matrix}

In step 3 of figure 2.2, the peak detection is performed by finding all local maxima and the associated peak endpoints (i.e., local minima) for each peak, and a signal-to-noise ratio is calculated. All local maxima (i.e., peaks) with signal-to-noise ratio above the threshold, which takes into account standard deviation of the signal, are kept as peaks. The mass spectra are sought and combined with the areas belonging to the peaks. The similarity index between the corresponding mass spectra is calculated to merge similar compounds along all available samples (step 4 in figure 2.2). This step enables one to represent each VOC as a single number in the data matrix across all measured samples. This number corresponds to the relative amount of the compound in the measured sample. Finally, the data matrix containing an observation in the row and relative amount of VOCs as values in columns is obtained (step 5 in figure 2.2). The label of each column (i.e., VOC1, VOC2 etc) corresponds to a scan number.

\subsubsection{Data preprocessing of MCC-IMS data}

\subsubsection{RIP detailing}

Each MCC-IMS chromatogram contains a characteristic structure called the reactant ion peak (RIP). This structure can be considered as a source of disturbance in a measurement, and it appears in the shape of a broad vertical line in each chromatogram. The signal descent on the right side of the RIP is RIP tailing. Therefore, the first step of MCC-IMS data pre-processing consists of RIP detailing (see figure 2.3, step 1). For that purpose, several methods have been proposed. For instance, one approach fitted a lognormal function to the mean of all spectra and subtracted this function from each spectrum in the chromatogram [45]. Another approach achieved RIP detailing by subtracting the $25 \%$ quantile intensity determined for each $1 / \mathrm{K} 0$ value over all spectra [46]. A technique here applied subtracts the vector of median values (median spectrum) from each IMS spectrum in the data matrix [47]. The consecutive stage aims to improve the comparability of IMS chromatograms by correcting baseline. This is done by subtracting the mean intensity of the pure noise region from all measured spectra [45].

\subsubsection{Denoising and baseline correction}

A very important part of the data pre-processing is to improve the signal-to-noise ratio. This is done by denoising and smoothing (step 2 in figure 2.3). For MCC-IMS 


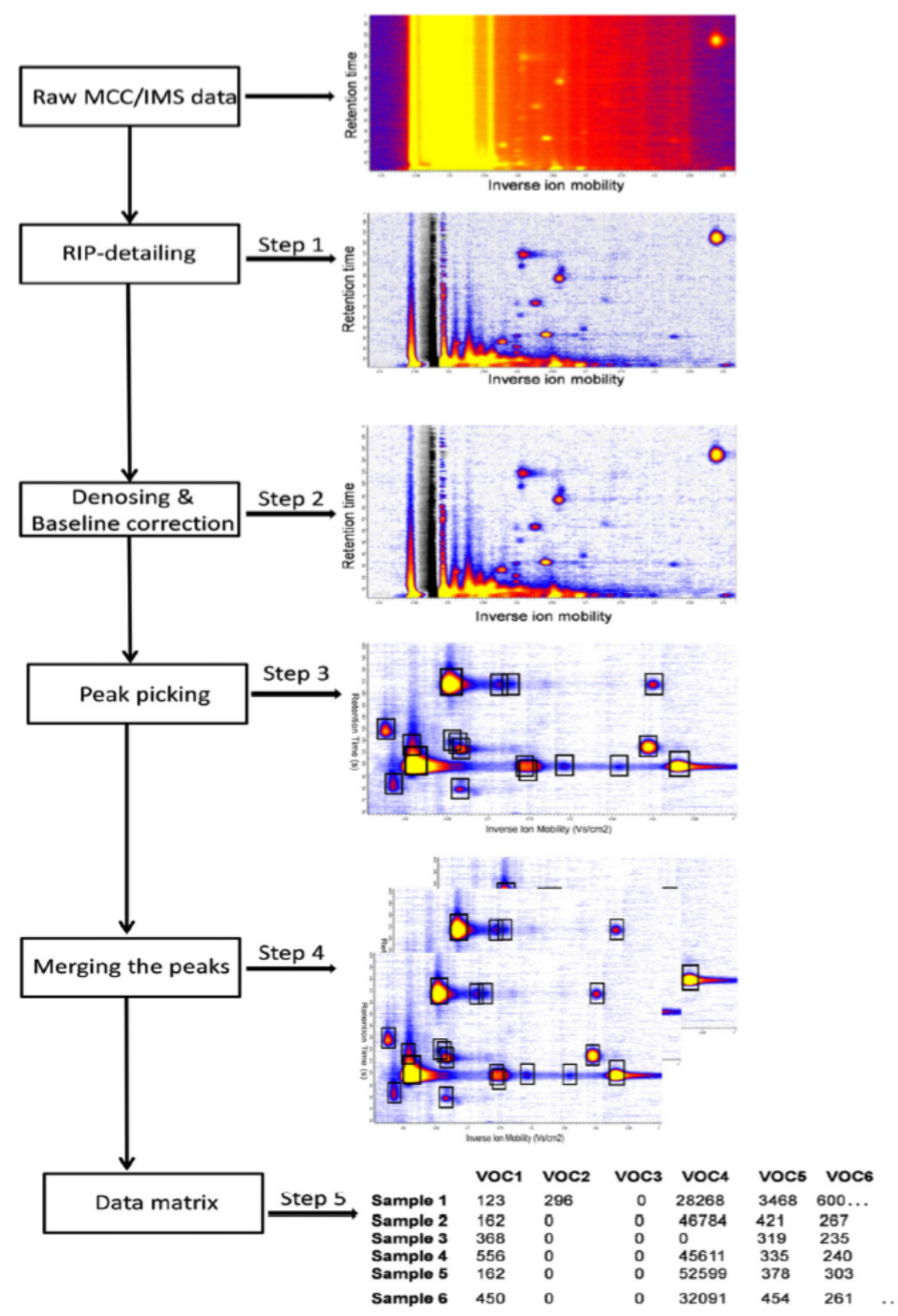

Figure 2.3: Example of a processing strategy of MCC-IMS data involving RIP detailing (step 1) and denoising and baseline correction (step 2), peak picking (step 3), merging the peaks (step 4) and building the data matrix (step 5). 
data, many different methods are available. In one of the first approaches (and presented here), a multiresolution analysis is performed, which includes discrete wavelet transformations on different levels of resolution for both denoising and smoothing [45]. The original chromatogram is reconstructed using the corresponding wavelet coefficients, which can be altered using hard and soft thresholding. To smooth the data, the coefficients corresponding to the high-frequency regions are eliminated (independently of the coefficient amplitude). Removing the low-amplitude coefficients regardless of frequency results in denoising. The resulting IMS chromatogram is reduced by at least $75 \%$ of the original data with negligible loss of information. A more recent approach proposed a pipeline of filters [46]. First, the median filter is applied for denoising, followed by a SavitzkyGolay and finally a Gaussian filter for smoothing.

\subsubsection{Peak picking and data matrix}

The last step of data pre-processing consists of peak picking. Several automated strategies are available such as merged peak cluster localization [47], growing interval merging [45], and wavelet-based multiscale peak detection [46], watershed transformation (WST) [46] and peak model estimation (PME) [48]. We focus on the most widely used approaches for MCC-IMS data and give their key ideas in the following.

The merged peak cluster localization consists of two phases: (1) the clustering and (2) the merging. In the first phase, each data point in the chromatogram is assigned to one of two classes, either peak or non-peak, using a clustering method similar to the traditional k-means, based on the Euclidean distance metric of the intensity values. In the second phase, neighboring data points that belong to the same peak are merged together. This procedure was first introduced for MCC-IMS data by Bader et al [47]. The simplicity of the method causes a few limitations such as the distinction of two neighboring peaks, where the signal intensity of the overlap is above the peakto-noise-threshold. Finally, each peak of the analyzed measurement is characterized by the centroid point, i.e., that data point which has the smallest mean distance to all other points in the peak region [49]. This approach is present in the commercial software package VisualNow (B\&S Analytik, Dortmund, Germany).

The WST approach for MCC-IMS peak detection mainly builds on the watershed method [50]. The IMS chromatogram is treated as a landscape including hills and valleys. The algorithm starts with a water level above the highest intensity followed by a continuous lowering of the level while uncovering more and more of the local maxima. In each step, the new uncovered data points are annotated by the label of adjacent labeled neighbors. Those data points that remain unlabeled are identified as a new peak and receive a new label. The highest data point among a set of new labeled positions defines the peak coordinate. Finally, the algorithm stops if all data points are labeled or the level drops below a defined threshold [46]. One of the software tools implementing this approach is IPHEx developed by Bunkowski [46].

Initially the PME was designed to describe peaks with statistical mixture models of parametric distributions, but turned out to be a new opportunity for high-quality peak detection. The expectation maximization algorithm is used to optimize the parameters of a mixture model from a given set of starting values. In general, all previously described peak detection methods are suitable to provide the initial 'seeds' 
for the algorithm.

Each peak is described by a model function consisting of two shifted inverse Gaussian distributions and an additional peak volume parameter. Finally, the set of model functions plus a noise component describe the whole MCC-IMS measurement. This approach uses continuous functions providing non-integer peak positions, which is presumably more precise than the discrete results of the previous methods. Kopczinski et al published an implementation of this approach [48]. It includes a seed-finding method based on finding roots in the first derivatives of both spectra and chromatograms [51].

Similarly to GC-MS, the final product appears as a data matrix containing observations in rows. The values in columns correspond to the relative amount of VOCs, i.e., their intensities (step 5 in figure 2.3). The label of each column (i.e., VOC1, VOC2 etc) represents retention time. Such a general representation of the data allows one to apply various machine learning techniques.

\subsection{Normalization, scaling and transformation}

\subsubsection{Normalization}

Normalization and scaling are usually the intermediate steps between the preprocessing of the raw data and multivariate statistical analysis. The aim of the normalization is to reduce the influence of unwanted systematic variations between measured samples, e.g. coming from the way the samples were collected and analyzed. Normalization is required when the total intensity differs between measured samples. The goal of normalization is to present each sample in the data in an adequate and consistent way. Therefore, normalization, or its absence, has enormous influence on the data analysis. If the normalization has not been applied in the correct way, this might obscure putative differences between studied cases. Normalization can be seen as a procedure where a scaling factor is estimated and applied to each measured sample.

Different methods are available on how the scaling factor is estimated, including the most commonly applied technique, total area normalization, which uses the total area of the chromatogram as a normalization factor. The application of this approach depends on the following assumption: the total profile is directly proportional to the total concentration of the sample. Moreover, it assumes that the total area is constant between samples. If this is not the case, this normalization should not be applied. Very closely related normalization is a total sum normalization which uses the inverse of the total area. The utilization of this method takes place when there is no straightforward way of estimating the total area, e.g. if instead of spectra, a digitalized version is available. The above normalization procedures take a full spectrum as the basis for calculating a normalization factor.

Additionally, several other types of normalization procedures are possible, in which only a part of the total spectrum or even a single compound is used for normalization. This selective normalization is for instance applied when there is an opportunity to include internal or external standards in the analysis. At present, the use of standards 
is hardly applicable in breathomics. The use of selective normalization is also opportune when some regions of spectra are to be removed, because of their high intensity and/or high variance. Furthermore, normalization to the largest peak in the chromatogram is commonly used since it is believed that it will provide comparable data for subsequent database searches, i.e., for identifying unknown compounds. However, this normalization is highly likely to introduce major errors if machine learning methods are applied since it introduces spurious correlation between compounds.

There are other types of normalization methods as well, for instance probabilistic quotient normalization [52], 'histogram' normalization [53] and group aggregating normalization [54]. Although not yet directly used in breathomics, they are suitable for removing undesired variations from VOC data. To date, several normalization approaches are available and reviewed by Bylesjo et al [55].

\subsubsection{Scaling and transformation}

MS-based technologies detect, in exhaled air, a large array of compounds and the relative abundance of the respective VOCs can vary by many orders of magnitude. Additionally, usually the higher the relative amount of a compound, the larger the variation in its measurements between samples. Therefore, in certain circumstances, it is desirable to scale or transform the data to correct for these differences in variations between compounds.

Numerous scaling procedures focus on different features of the data and consequently each approach has advantages and disadvantages. The first scaling approach is applying meancentering which is nothing other than subtracting the mean value from each measured compound. The outcome causes relative amounts of the VOCs to fluctuate around zero instead of the mean. This simple procedure corrects for the differences between high and low abundant compounds. Meancentering is usually used in combination with other scaling approaches. One of them is autoscaling, also called unit variance scaling. Autoscaling scales all compounds to unite variance by using standard deviation as a scaling factor. This means that by analyzing autoscaled data, each VOC has equal importance independently of its abundance. Since this scaling gives equal importance to all compounds, it should not be used for noisy (i.e., poor signal-to-noise ratio) data. The intermediate solution is Pareto scaling where the square root of the standard deviation is used as a scaling factor. In that way, large changes decrease more than smaller ones. Another scaling method that uses standard deviation is vast scaling [56]. This scaling approach uses the coefficient of variation. Thus, the importance of compounds that vary a lot is diminished while that of compounds with small relative standard deviation is increased. These scaling methods use the standard deviation as the dispersion measure.

It is also possible to use the differences between the maximal and a minimal amount of a certain compound in the measured set of samples as an indicator of data spread. This is range scaling [58] and since only two values are used as the scaling factor, it makes this approach sensitive to outliers. A last scaling method is level scaling, which uses the average value of each compound as a scaling factor. Thus, the resulting scaled data represent changes in percentage compared to the mean concentration. This scaling method is of interest when relatively large biological changes are of interest. 
It is possible, for each scaling method described above, to use a more robust measure, i.e., the median instead of the mean. This allows diminishing the impact of outliers present in the data [57].

Next to scaling methods, transformation [60] of VOC data can be performed before data analysis. Transformation techniques belong to nonlinear conversion of data, such as log transformation, BoxCox transformation [34], rank transformation [58] and power transformation. The main aims of transformation are symmetrizing data distribution (from a tilted distribution), stabilizing the variance and making the distribution closer to normal. As mentioned previously, transformation allows removing the effect of heteroscedasticity in the data.

\subsection{Multivariate statistical methods}

Breathomics, which comprises typically metabolomics studies of exhaled air, generates huge data sets, where number of variables (compounds) exceeds the number of samples. This implies dealing with 'fat matrices'. Moreover, collinearity between measured variables exists. In many cases, breathomics data are, to some extent, sparse, i.e., a compound is present only in e.g. $20 \% 30 \%$ of the samples. In addition, breathomics data are often non-normally distributed. These issues have to be taken into account when applying machine learning techniques [59]. Machine learning provides a plethora of methods to explore and understand complex data and thus obtain valuable information on biological changes. Usually the multivariate analysis starts with data exploration and discovery. This part of the analysis is a blind and unsupervised approach and thus gives an unbiased view on the data. Typically, multivariate statistical analysis continues with supervised analysis in which a priori knowledge of the data structure is utilized.

Some of the main characteristics of machine learning methods described are summarized in figure 2.1. It contains sensitivity to common issues such as outliers, scaling or respect of a normal distribution. The possibility to evaluate the compound's importance from the constructed models is crucial for interpretation, yet not all machine learning can provide this information, as shown in figure 2.1. Finally, we list applications of these methods to VOC studies.

\subsubsection{Unsupervised analysis}

One of the most widely used explorative analysis techniques is principal component analysis (PCA). This workhorse selects a small number of linearly uncorrelated PCs that explain the majority of the variation in the data. The first $\mathrm{PC}$ is defined by the direction of the largest possible variation in the data. Each following PC is selected as the most varying orthogonal component [79]. PCA results in two matrices known as scores and loadings. Scores represent new coordinates for the samples and loadings show the way in which old compounds are linearly combined into PCs.

The outcome of PCA can be visualized using a score plot and a bi-plot. In the score plot, each breathomics sample is represented by a single point and demonstrates the relations (i.e., similarity) between all samples in breathomics data. Those that 


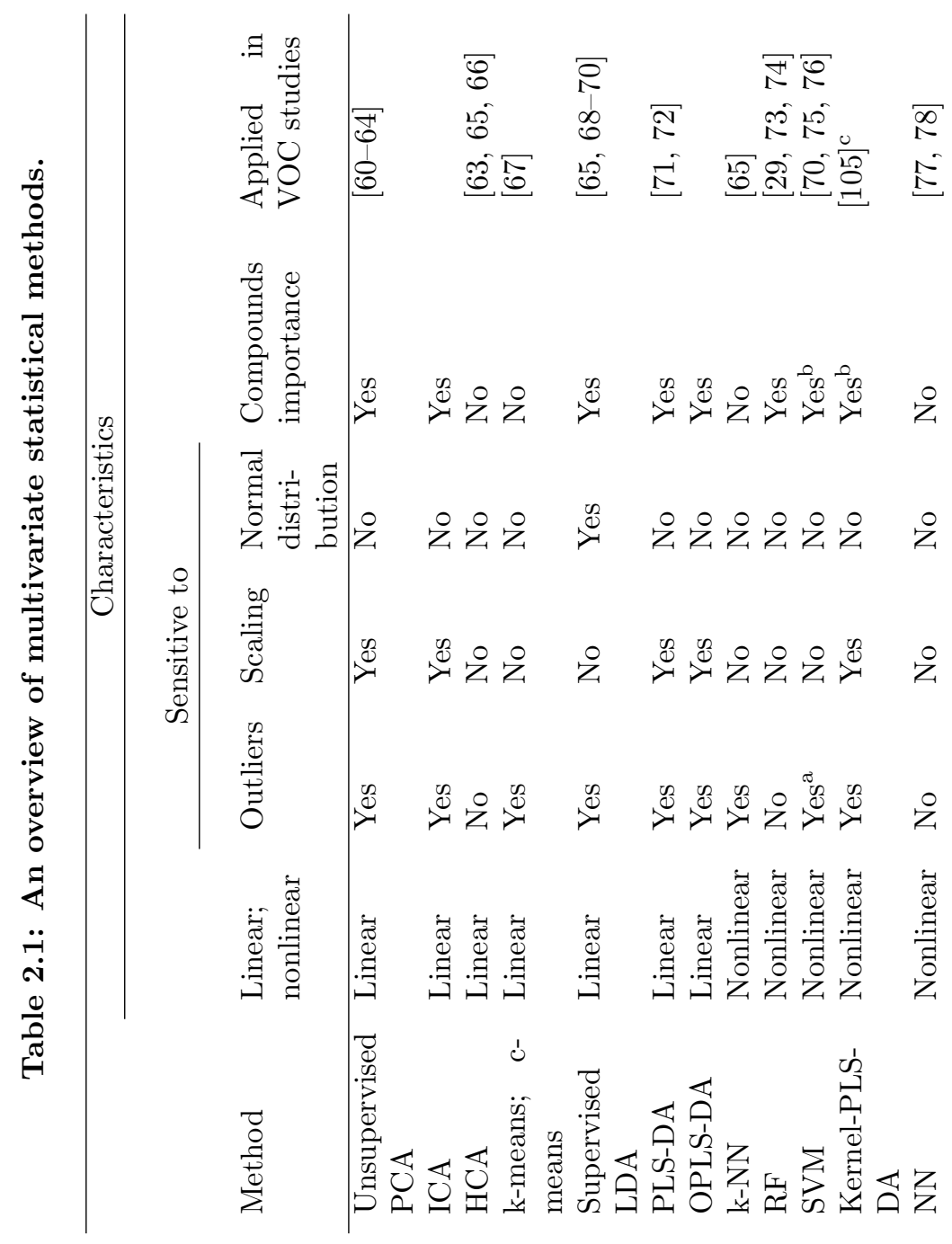


lie close to each other have similar properties (i.e., VOC's profile), while points that are distant have different properties. figure 2.4(a) shows an example of a score plot of simulated breathomics data. In the bi-plot (figure 2.4(b)), information on the relation between compounds and the difference in relative abundance between clusters of samples is presented. Similarly to the score plot, each point corresponds to a single sample and each line to a compound. Correlation between compounds is reflected by the angle between compounds (i.e., between lines). Two compounds are positively correlated if the angle is small, which implies that their relative abundance changes in the same way. An angle close to $180^{\circ}$ or $90^{\circ}$ indicates an anti-correlation or absence of correlation, respectively. figure 2.4(b) shows an example of a PCA bi-plot.

The PCA methodology is well suited to summarize high-dimensional data, but was not developed to find the direction or pattern of variables that best separates classes of objects. Note that the obtained PCs can often explore clusters in the PCA score plot since homogeneous groups of objects are usually distributed along the directions of largest variance. Nevertheless, if the class information is not associated with these directions, the PCA does not uncover it [80]. The variance of data is sensitive to the presence of outliers and consequently the detection of outliers is crucial in multivariate analysis. PCA is capable of identifying only certain outliers, i.e., good leverage samples (lying far away from the majority of all samples). PCA is not suitable to detect orthogonal outliers because these outliers fall within the PCA space for the majority of the samples. To overcome this drawback robust PCA can be used since this provides a set of robust factors which are not affected by outliers. Many types of robust estimators have been proposed, and their description can be found elsewhere [81].
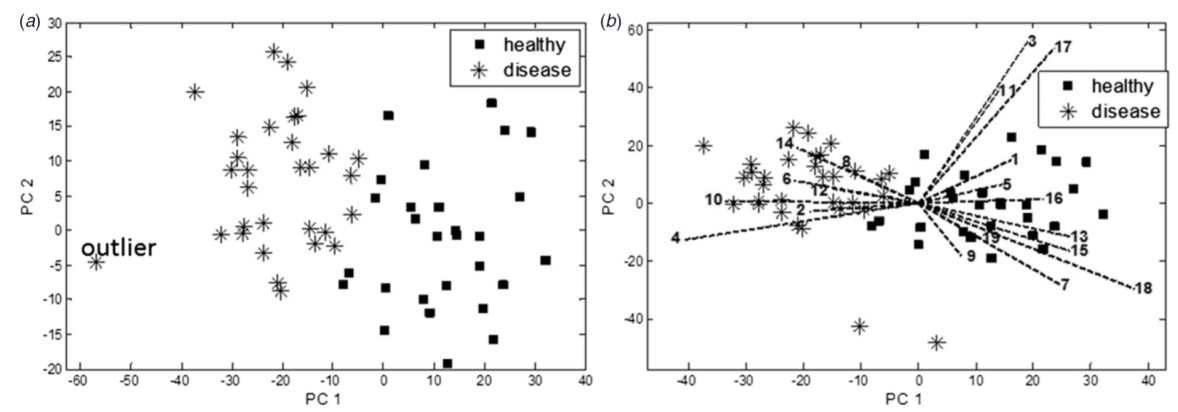

Figure 2.4: An example of PCA: (a) score plot; the points are coded for class membership (squares for healthy and stars for disease). Two groups of points show distinct clusters, separated along PC1. (b) Biplot; every point corresponds to a single breath sample and the lines to the VOCs. The levels of compounds facing in the direction of disease cluster (i.e., numbers 1, 3, 5, 7, 9, 11, 13, 15, 16, 17, 18 and 19) are elevated in the breath samples of this group of individuals. The compounds that point in the opposite direction are reduced in the breath samples of disease group: numbers 2, 4, 6, 8, 10, 12 and 14 .

Closely related to PCA is the independent component analysis, which assumes that 
the obtained data are generated by mixing a number of independent sources. This results in a set of latent variables $(\mathrm{LVs})$, which are constructed to be statistically independent. While mostly applied in the area of signal processing, it has gained more popularity in chemistry and might also be interesting for the field of breath analysis [80].

Another popular unsupervised method to analyze multivariate data is clustering [12]. Clustering methods are based on similarity measures (e.g. determining multivariate distances such as Euclidean, Manhattan, Mahalanobis, Minkowski distance or correlation) of the whole breathomics data set. One often uses a method to test if natural groupings (clusters) are present in the breathomics data. For instance, if the clusters are compact in the breathomics data, then it may be sufficient to use only a part of the available information in the original data by choosing a group representative. The distance measured between samples may be overestimated due to sparsity of breathomics data. This means that rare (sparse) compounds affect the distance between samples, making it bigger. Therefore, it is worth removing compounds that are present only in a few samples. The method named hierarchical cluster analysis (HCA) is, due to its simplicity, the most widely used clustering approach. It is based on the iterative calculation of distances between the measured samples. In each step of the iteration, it merges the two closest samples until all samples belong to the same cluster. The calculated multivariate similarity/dissimilarity can be represented as a tree called a dendrogram. Various ways of recalculating the distances while merging two samples exist and the most common recalculation methods are based on the average linkage (average distance), single (minimum distance) or complete linkage (maximum distance) [79]. The choice of the linkage method largely influences the result of the clustering. Single linkage is prone to a phenomenon called the 'chaining phenomenon', wrongly merging close clusters. Even if the average linkage approach is the most stable one, a closer look at the resulting dendrogram is indispensable to verify the ultimate result. Clearly HCA works best when a hierarchical structure is present. By determining the similarity cut-off, which splits the dendrogram into separate clusters, HCA can be used for classification. However, the main disadvantage of $\mathrm{HCA}$ is that it does not deliver information about the compounds which are responsible for the resulting clustering. To overcome this drawback, coclustering (also known as bi-clustering) has been developed. This method simultaneously clusters data in its rows and columns (samples and compounds) [82, 83].

Next to HCA, k-means clustering (a partition method) is another approach commonly applied. It makes use of a completely different way of defining clusters in the data. The k-means clustering selects a set of centroids (representing clusters) in such a way that the overall distance of all samples to the centroids is minimized. Note that a number of centroids have to be selected by the user. In the second step, each sample point is assigned to the closest centroid. In step 3, the new centroids are defined as being the average value of all samples assigned to a given cluster. Next, steps 2 and 3 are repeated until convergence (i.e., no more changes in cluster content) or maximal number of iterations is reached [79]. The random centroids can lead to local maxima and thereby produce non-deterministic results. Therefore, to find the optimal set of centroids and thus the optimal solution, the algorithm is repeated with different starting points. 
Unlike HCA, one solution is obtained with a predefined number of clusters. If the algorithm is repeated for the same data set but with another number of defined clusters, there might be little or no relation between the two products. An adaption of $\mathrm{k}$-means is fuzzy c-means [84] which, instead of assigning each sample to one cluster, gives the probability of belonging to each cluster.

A drawback of clustering methods is the vulnerability to outliers and that they are not suitable for very large and complex datasets. More sophisticated approaches, like distribution-based (e.g. Gaussian mixture models [79]) or graph-based clustering methods (spectral or transitivity clustering $[85,86]$ ), are often more suitable for large and more complex data sets. Nevertheless, most of them require more in-depth knowledge of the principles of the methodologies and the structure of the data, to be able to choose the right approach and correctly set the parameters.

Finally, all clustering results should be assessed by internal or external evaluation criteria. Internal criteria like the Silhouette value are dependent on the underlying data themselves and favor clustering with high inner cluster similarity and high distance between them. However, if group information is available, it can be used to estimate the similarity of the clustering to the real grouping, also called external criteria (e.g. F-measure, Rand measure).

Unsupervised methods have found several applications in breathomics studies [60, $68,87-89]$. In most of the studies, PCA (and similar factor analysis methods) is used for exploring and reducing multidimensional data. Clearly, PCA is a perfect choice for such purposes. However, it is known that the outcome of PCA depends on the applied scaling and the eventual presence of outliers; these issues are barely mentioned in most breathomics studies.

\subsubsection{Supervised analysis}

As said previously, supervised methods use a priori knowledge about the obtained data and can be supplied as e.g. patient labels, treatment groups or subjects exposed to certain compounds. These methods use this knowledge to learn rules and patterns related to the classes in the data, which are then utilized to predict the classes within newly obtained data sets. The main aim of all supervised techniques is to find the relation between a matrix of predictors (VOCs) and vector (or matrix) of responses (e.g. class membership). An extremely relevant part of supervised methods is the validation of the predicting algorithms, which can be either cross-validation (CV) within the existing dataset or ideally within a newly independently sampled dataset. When a supervised model is built on a dataset containing more compounds than samples, it is easy to obtain a perfect fit of data resulting in a high prediction of classes. However, this may be an optimistic impression of the original dataset and can be the result of overfitting. To avoid this phenomenon of overfitting, thorough validation of the supervised model is mandatory. This implies that the supervised method is first applied to a training set and then validated by means of a test set. This aspect will be discussed later in section 6 .

A wide range of supervised methods for linear and nonlinear problems is available. Here, we focus on the most popular ones. 


\subsubsection{Linear statistical techniques}

A regularly applied method in breathomics is linear discriminant analysis (LDA) [69, 90-93]. This technique tries to find a linear function based on original compounds, which can be used to separate the studied classes. Note that LDA can be employed with numerous discriminant rules [11, 94], and can be applied to data where more than two classes have to be discriminated. In the case of LDA, K 1 discriminant functions (where $\mathrm{K}$ is a number of classes) are always calculated, which are subsequently used to assign group memberships. LDA is a fast and powerful technique with no necessity of optimizing parameters. However, it has several limitations. First of all, it assumes a normal distribution of the data. This is, however, very often not the case in many breathomics studies. Breathomics data are usually not normally distributed; therefore, they should be first transformed, for instance by a logarithmic function, into normally distributed data. LDA can be only applied if the number of samples is larger than the number of measured compounds. Typically, breathomics data contain more compounds than samples and consequently LDA cannot be directly applied. Obviously to overcome this limitation, many solutions have been proposed. The most straightforward is to apply an unsupervised method like PCA for data reduction and then use LDA on a score matrix with the relevant number of PCs (i.e., the ones that explained most of the significant variance). The number of PCs included in the LDA model has to be optimized, and this can be achieved via the procedure called leave-n-out CV (LnOCV).

The disadvantage of using PCA to reduce the data is the possibility of losing relevant information directly related to the classes. Again to overcome this problem, recent developments of LDA have been proposed by Nrgaard et al [95]. LDA gives the possibility to visualize not only differences between classes (by using a score plot of the discriminant function), but also to obtain a rough idea about the compounds' contributions related to these differences. This is done by comparing which compounds have statistically different means across the investigated classes. LDA is especially suitable for data structures in which low multicollinearity of compounds exists. Therefore, LDA is not the most suitable technique for breathomics data due to their comparably high multicollinearity.

Partial least-square (PLS) is a method originally developed for quantitative analysis in regression problems [96]. Soon, however, it became a very popular technique for classification and thus was called PLS discriminant analysis (PLS-DA). This method, similar to PCA, is an LV approach and thus it assumes that the data can be well estimated by a low-dimensional subspace, i.e., by LVs. Since, like LDA, it is a linear technique, new dimensions are calculated as linear combinations of the original compounds. PLS-DA tries to maximize the covariance between a dummy class vector $\mathrm{Y}$ and a data matrix $\mathrm{X}$.

A very important advantage of PLS-DA is its ability to cope with highly collinear data. Thus, it is a very suitable technique for breathomics data. It gives very good results for two-class classification problems and is to be preferred above LDA. When more than two classes have to be discriminated, an adaptation of PLS-DA (called PLS2-DA) can be applied. However, PLS2-DA often gives inferior results compared to multiple binary PLS-DA models (i.e., one class versus the rest). An overview of 
different statistics used to evaluate the performance of PLS-DA has been described previously [97].

PLS-DA requires the optimization of model complexity (i.e., number of LVs used in the model). For two-class classification problems, the most robust way is to calculate the number of misclassifications (NMCs) (i.e., the sum of false positive and false negative) within the CV procedure. A more detailed description of CV can be found later in section 6. The pseudo-code for $\mathrm{LnO} \mathrm{CV}$, for data matrix $\mathrm{X}$ (repeated for each $\mathrm{n}$ objects (i) in X), can be summarized as follows.

\section{Beginning of LnOCV}

1. $\mathrm{n}$ samples (i) are removed from the training data matrix $\mathrm{X}$ (with $\mathrm{m}$ samples and $\mathrm{p}$ compounds, $\mathrm{m} \mathrm{p}$ ).

2. Scaling of the data matrix $X$ with remaining samples (of size $m n ~ p$ ).

3. Scaling of removed samples (i) with mean and standard deviation delivered from the data matrix $\mathrm{X}$ with remaining samples.

4. Fit PLS-DA model to the scaled data matrix $\mathrm{X}$ with remaining samples (size $\mathrm{m}$ $\mathrm{n} \mathrm{p).}$

5. Classify removed sample (i). End of LnOCV

6. Calculation of NMC by comparing predicted class vector with real class vector $\mathrm{Y}$

7. Model complexity $=\min (\mathrm{NMC})$

This procedure is repeated until every sample is left out once. The model complexity can be expressed as a plot where the x-axis corresponds to the number of L- and the y-axis to NMC. The optimal model complexity is usually the first clear minimum on the NMC.

PLS-DA also provides information about the compound relevance. Using this method, we can distinguish the studied classes but also find which compounds are responsible for it. The information about class separation can be visualized in the score plot. Note that using only a PLS-DA score plot to present separation between classes is invalid. Let us consider a random data set of size e.g. 80 individuals (40 disease and 40 healthy) and 800 VOCs. Using PLS-DA to discriminate these random data into two groups almost always gives a PLS-DA score plot with perfect separation between the two arbitrary classes. This implies that PLS-DA is a very powerful technique, but it is prone to overfitting. Thus, when applying it to breathomics data, a validation scheme has to be employed.

The PLS-DA model should be evaluated based on prediction ability on a test set (to be discussed later). A minimum of the most relevant compounds can be obtained from the vector of the regression coefficients (the higher the most relevant). The importance of compounds can be shown as a bar plot of regression coefficients. In figure 2.5(a) is an example of regression coefficients obtained from a PLS-DA model performed on simulated breathomics data containing 80 samples and 800 compounds. 
As can be seen, several compounds have low regression coefficients (both negative and positive), implying unimportance in the classification model. Four VOCs, 25, 355, 494 and 607 , have the highest negative value of regression coefficients. Therefore, they are the most discriminatory compounds. However, by looking at regression coefficients, optimal threshold selection is not straightforward. Therefore, several other ways of measuring the compound's importance within PLS-DA have been developed.

One of them, called variable importance in projection (VIP), is a summary of the importance for the projections to find a number of LV [98]. Compounds with VIP superior or equal to 1 are considered significant in the PLS-DA model. The higher the VIP score, the more important the compound. figure 2.5(b) shows the values of VIP for the PLS-DA model shown in figure 2.5(a). Only 20 compounds have VIP score superior to 1 (see threshold indicated as black horizontal line) and form a set of the most relevant compounds in the PLS-DA model. Note that four VOCs have VIP score $i 4$ and that these are the compounds with the highest regression coefficients. The remaining compounds (with VIP score inferior to 1 ) do not deliver any additional information and, therefore, are considered as irrelevant. Another way of measuring is called selectivity ratio proposed by Rajalahti et al [99], which calculates the compound's importance by using residual and variance explained for each compound. Selectivity ratio delivers for each compound a value indicative of the discriminative power. A high value means a high discriminatory compound while low values mean irrelevant VOCs.
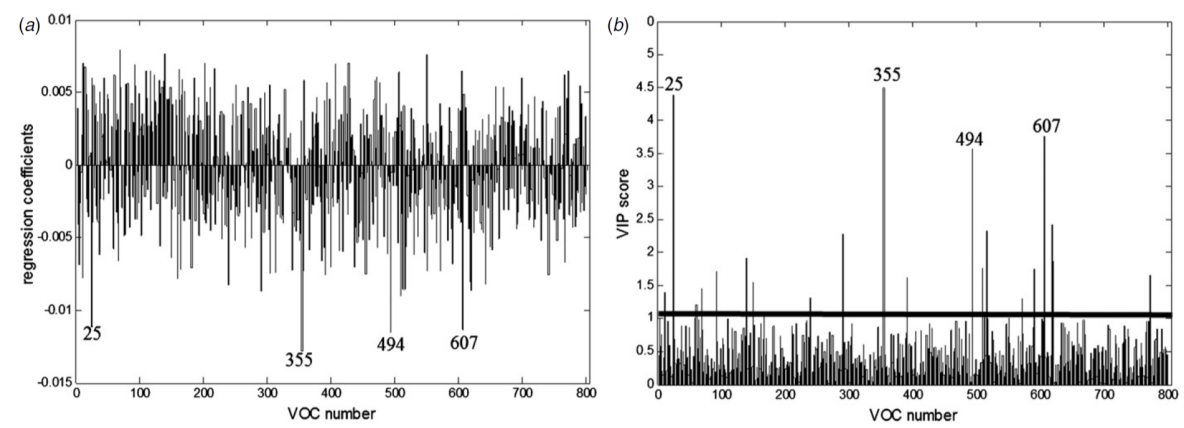

Figure 2.5: The example of compounds importance expressed as (a) regression coefficient obtained from a PLS-DA model; (b) VIP score calculated for the PLS-DA model shown in (a). The threshold indicates the most important VOCs (i.e., VOCs with VIP score above 1). The VOC numbers 25, 355, 494 and 607 have the highest negative values of the regression coefficient, as well as VIP score. These four VOCs have the highest importance in the PLS-DA model.

In breathomics, PLS-DA was successfully applied to discriminate healthy control children and allergic asthmatic children [71], distinguish different smoking-related diseases [87], detect pseudomonas aeruginosa in sputum headspace [100] and identify lung cancer citeNatale2003. PLS-DA is an adequate technique for most breathomics data. However, proper validation is crucial for this method. In most cases, PLS-DA models are validated via LnOCV (where $\mathrm{n}$ is between 1 and 10). In this validation, the same 
samples are used to optimize the classification model and assess the general predictive power of the final model. This validation produces a biased and over-optimistic estimation of the classification error. If one uses LnOCV, this translates practically in building multiple PLS-DA classification models. These models are not necessarily similar: they may involve different sets of VOCs. This indicates that the ultimate classification model is unstable with respect to the dataset. Therefore, LnOCV will possibly produce a bad final model. The only way of providing evidence that the PLSDA classification model is unquestionable and robust is to use an independent test population or bootstrapping. Note that this issue also holds for nonlinear methods described later in the paper.

Recent development of PLS-DA, orthogonal PLS-DA (OPLS-DA) [101] is based on dividing the overall variation in the data into response predictive (i.e., linearly related to the class vector) and orthogonal (i.e. uncorrelated to the response). OPLS-DA is suitable for highly collinear data, such as in breathomics. The main advantage of OPLS-DA is the interpretation of the model since irrelevant information is filtered out. This allows users to focus only on the first LV to interpret the results. This is particularly interesting in the case of breathomics data containing thousands of VOCs. The unrelated variation, filtered out by OPLS-DA, is called structure noise and is present in the data due to differences in e.g. diet, age and gender. The prediction power of PLS-DA and OPLS-DA is comparable. Similarly, to PLS-DA, it is possible to apply OPLS-DA to a more than two class classification problem.

\subsubsection{Nonlinear statistical techniques}

The methods described in the previous sections are very well equipped to investigate linear relationships between variables (e.g. compounds) and class label (e.g. disease status). However, these approaches are less suited to investigate the nonlinear relations that may be present in complex biological systems [102]. Nonlinear relations might originate from different metabolic backgrounds of hidden disease subgroups, variations in disease stages or individual patient conditions or medication [103]. In the following, we will introduce different forms of nonlinear statistical learning approaches, namely tree-based methods, neural networks and kernel-based methods. Note that linear methods should be the starting point in multivariate statistical analysis. Linear techniques offer several advantages, such as simple interpretation of the outcomes, fewer parameters to optimize and fast calculation. Nonlinear techniques are usually more powerful in terms of prediction accuracy. However, most methods have complex interpretation of the outcomes and are computationally intensive to calculate. Therefore, we advise to carefully inspect the data and use them only when linear methods are not sufficient to deal with complex biological systems.

The first technique described here is k-nearest neighbor (k-NN) [104], where $\mathrm{k}$ is a parameter to be determined. This non-parametric technique, similarly to the clustering methods described previously, is based on the concept of multidimensional distances. However, these distances between samples are used in order to take a formal decision on the assignment of a sample to a class. To do so, the distances are calculated between the new sample and a set of previous measurements. This collection of known samples forms the training set. The points that are close to each 
other are similar. Therefore, in case of $\mathrm{k}=1$, one can infer that a point lying close to a training sample belongs to the same group as the training sample. This idea can easily be generalized by considering more than the closest training sample $(\mathrm{k}=1)$ such as the three $(\mathrm{k}=3)$, five $(\mathrm{k}=5)$ or seven $(\mathrm{k}=7)$ closest training samples. If $\mathrm{k}>1$, the classification of samples is not so straightforward. Usually, a majority vote is used. The main advantage of $\mathrm{k}-\mathrm{NN}$ is its ability to construct a very efficient model describing complex nonlinear boundaries between the groups. The main drawback is that it is a computationally intensive method requiring recalculating the distances to all samples for each new measurement. Moreover, it does not deliver information about significant compounds. Since k-NN uses distances between all pairs of data points for decision making, for the sparse breathomics data it may cause the same problem as in clustering techniques.

The most straightforward nonlinear methods are based on decision trees.

An example is the classification and regression tree (CART) method proposed by Breiman [105]. The goal of CART is to find exclusive regions in the data that contain homogenous subsets of the data (i.e., defined classes). The outcome of the CART is presented as a binary tree, constructed by recursively dividing the samples from a parent node into two child nodes. Each node is described by a simple, logical rule based on one compound. Note that, at each split, a different compound is used; nevertheless the same compound may appear more than once in a single tree. The splitting continues until the similarity of the samples within each node is the highest one or nodes contain the minimum number of samples (this has to be specified by the user). The splitting compounds intuitively serve as the most important set of features. Nevertheless, the strong tendency of overfitting results in a large variance between single decision trees on slightly different data sets. Therefore, the combination of ensemble trees reduces the overall variance of the models and thereby significantly increases the performance.

This is done in the random forest (RF) methods as proposed by Brieman [106]. RF builds a large collection of de-correlated trees (weak classifiers) each on a randomly selected subset of the original samples (called bootstrap aggregation (bagging)) and on randomly selected subsets of compounds [79]. The error rate of the RF model depends on two things: firstly the correlation between trees and secondly the strength of the individual trees. Next to the RF method, the boosted tree algorithm can be used. This tree-based technique iteratively appends weighted trees to the model, while mostly focusing on the prediction of the previously misclassified samples. In general, this approach is called boosting, while AdaBoost is the most widely used variant [79].

Note that the goal of RF and boosted trees is very similar, i.e., both build many weak classifiers as different as possible from each other. However, RF focuses on randomization while boosted trees focus on misclassified samples of previous models and thus build a different model. Advanced ensemble tree approaches are based on solid statistical theories and hence outperform other methods on many data sets in terms of classification accuracy. In addition, they provide comprehensible importance estimations for the single features which allow for supervised dimension reduction and support the direct abstraction of potential biomarker patterns $[47,107,108]$. RF is suitable to deal with both linear and nonlinear problems. It is a non-parametric approach, so it does not assume any distribution in the data. It is capable of dealing 
with highly collinear breathomics data. In addition, the RF technique is resistance to the different type of outliers and mislabeled samples. The main disadvantage of RF might be large computational time when applied to breathomics data.

Among the many nonlinear techniques, the so-called kernel-based models are the most popular ones. Here, we will discuss support vector machines (SVMs) and kernelPLS-DA. Kernel-based models require transformation of the data via specific functions called kernels. The key idea of kernel transformation is to map the nonlinear problem in the original data into a higher-dimensional feature space, corresponding to a reproducing kernel Hilbert space (RKHS), in such a manner that the problem becomes linear and thereby easily solvable.

The kernel function calculates the inner products of the original compounds in the RKHS without the need of transforming the whole data set, which is called the 'kernel trick' or kernel property. For more details, we refer to [79]. By mapping the original data of size ( $\mathrm{m} \mathrm{p}$ where $\mathrm{m}$ is the number of samples and $\mathrm{p}$ is the number of compounds) into the feature space, a kernel matrix of size $\mathrm{m} m$ is obtained. The kernel matrix has to be positive semi-definite and likely there are many kernel functions which fulfil this constraint. The simplest kernel function is the dot product of the data matrix. This is the linear kernel and thus is considered as linear approach. Next to the linear kernel, a widely used kernel transformation is the radial basic function. This function has one parameter to tune, i.e., the width of the Gaussian function. Note that this function is very robust, so it is suitable for any nonlinear problem.

The first kernel-based method described here is SVM. In the optimal case, the SVM finds a hyperplane that perfectly splits two classes while maximizing the thickness of the margins, which correspond to the distance of the plane to the closest data point from either class. To split overlapping classes, the margin is maximized while penalizing the points that lie on the wrong side of the margin. In contrast to LDA, only the points on the boundary or the wrong side of the margin support the split and are therefore called 'support vectors'. The single SVM without the use of a nonlinear kernel belongs to the class of linear methods and is therefore also called 'linear SVM'. SVM is suitable only for two-class classification problems. It is a very powerful method. However, the solution can be sparse since it is built on a limited number of samples, i.e., support vectors. Kernel-PLS-DA has comparable prediction ability to SVM. In contrast to SVM, in the kernel-PLS-DA algorithm, the solution is made using all available samples [103].

In general, kernel methods have a strong discrimination power, but the results are highly dependent on the chosen kernel function. Furthermore, SVM has been proven to be very insensitive to perturbations and outliers, especially on data sets with a large number of samples since only a few support vector points influence the boundary. This group of methods has been used in various publications of breath analysis and metabolomics, due to their advantages [103, 108-110]. The disadvantage of kernelbased methods is the loss of compound information, since after kernel transformation, the new data set has a dimension of $\mathrm{n} n$ (where $\mathrm{n}$ is the number of samples). Thus, the direct interpretation of discriminatory compounds in the kernel-based models is not possible. This has been recently solved by Krooshof et al [111] by applying a procedure based on a nonlinear bi-plot. In addition, it is possible to obtain compound ranking by applying recursive feature elimination [112] within SVM. The ranking reflects the 
importance of the compounds in the classification problem. Interpretation of kernelbased techniques is still a bottleneck. Although, several solutions are available, the interpretation of highly nonlinear classification problems remains elusive.

A recent paper by Amato et al [113] pictured the growing importance of artificial neural networks (ANNs) in medical diagnostics. The key idea of ANNs is to derive a set of novel features ('hidden' layer) as linear combinations of the input variables and combine these features in a nonlinear way to model the target label. The general structure of an ANN contains an 'input' layer, one or more of the previously mentioned 'hidden' layers and finally the 'output' layer. This method is very powerful and flexible to model nonlinear problems. However, interpretation of the effects of a compound is challenging. The most common 'vanilla' neural net contains only one interlayer and is called the single-layer perceptron. Variants of this approach have been applied in various areas of breath analysis, for instance for analyzing sensor array results [114], MS data [77], and IMS measurements [108].

Various multivariate statistical models can be built using the same training data set. In the literature, several papers have demonstrated integrated systems, including several machine learning methods, for VOC-based supervised classification into patient groups $[108,115,116]$. The main aim of applying multiple machine learning techniques to a single dataset is to provide a comprehensive and robust description of the data. Moreover, different techniques enable selecting various informative attributes from the data. Clearly, the benefits of applying multiple techniques depend on the underlying problem. Using a set of linear and nonlinear techniques to classify patients groups, most likely nonlinear methods deliver the highest accuracy (e.g. SVM, kernel-PLS-DA). However, as mentioned earlier, nonlinear methods are powerful, but the interpretation of the findings is very often difficult. One should always decide which aspect is preferable: interpretation or accuracy. Obviously, if the data are expected to be nonlinear, the linear modeling can be expected to perform less well. One may also apply several techniques and compare them to determine the most suitable one for the given data set.

\subsection{Data fusion}

In the field of breathomics, it is very common to study diseased versus non-diseased groups using various analytical platforms e.g. GC-MS, electronic nose or MCC-IMS. However, it is not widespread to combine the outcomes of different platforms by data concatenation or data fusion. Nowadays, data fusion has become very popular in the field of metabolomics. Therefore, we believe that data fusion will also be of value for the breathomics world.

Data fusion, sometimes called data concatenation, is an approach combining data coming from different high-throughput machines (platforms). It gives the possibility to obtain a better description of the studied problem. The idea behind fusion of data lies in the assumption that each analytical platform has different strengths and weaknesses with respect to the measured compounds, analytical reproducibility and sensitivity. Thus, by joining, the outcomes of dissimilar analytical technologies may have a positive influence on data analysis and the produced results. Moreover, a more 
robust description of the study population is gained than can be obtained by using data from a single platform.

Fusion can be performed on (i) different analytical techniques, i.e., one sample measured by different techniques or (ii) different biological samples, e.g., breath air and breath condensate measured by the same analytical platform. Hall et al have described three different strategies for data fusion [117] as illustrated in figure 2.6.

The first strategy in data fusion, referred as low-level data fusion (figure 2.6(a)), belongs to one of the most straightforward ways of concatenating data coming from different sources. It involves the joining of various data by putting them next to each other. Consequently, the new data matrix contains the same number of samples as the original sets of data and the number of compounds is the sum of the individual compounds. When the new data matrix is analyzed no prior analysis is done, such as a reduction of a number of compounds.

Mid-level data fusion (or feature-level, figure 2.6(b)) is another approach in which each original data set is first treated individually for data pre-processing, scaling, relevant compounds selection and dimension reduction via multivariate analysis, e.g., PCA. After this, the significant compounds or the reduced dimensions are only combined and analyzed.

The last type is high-level data fusion, which combines the independently generated predictive algorithms of the classification models obtained for each data set [84]. So the classification model is first calculated for each data set separately and next the outcome of the model is fused. It is important to decide which method for high-level fusion will be used, and the most straightforward way of combining the outcomes (figure 2.6(c)) is to use the rule of the majority vote [118]. In this approach, each of the constructed models predicts a class membership for each sample. The final class membership is obtained by the majority of the models. This methodology can be used only when more than two classification models are built. It is also possible to use weighted fusion, i.e., each prediction gets a weight [119]. More advanced methods such as naive Bayes are available as well [118].

The question, 'which data fusion strategy should be used?', is highly dependent on the aim of the study. If prediction accuracy is the goal of the study, then highlevel data fusion is the most suitable selection. However, the model generated by high-level data fusion is hard to interpret, and significance of compounds is often not straightforwardly indicated. Another disadvantage of high-level fusion is the loss of correlation patterns between measurements in the different data sources. In low-level and mid-level data fusion, the information about relevant compounds and the relation pattern between measurements is preserved.

A special case of data fusion is multiple kernel learning (MKL) originally pioneered by Lanckriet et al [120] and Bach et al [121] as an extension of a single kernel to combine multiple kernels in SVM. The principle of MKL is to combine kernel matrices into a single kernel using their parameterized linear combination. Recently this approach was adapted by Smolinska et al to k-PLS-DA [103]. In MKL, each kernel matrix, delivered from different analytical platforms, obtains a weight. The weight is optimized by different regularization methods, such as the L1 or L2 norm. These weights are next used in the linear combination of kernel matrices. The bigger the weight, the more important the platform associated with it. The aim of data fusion 


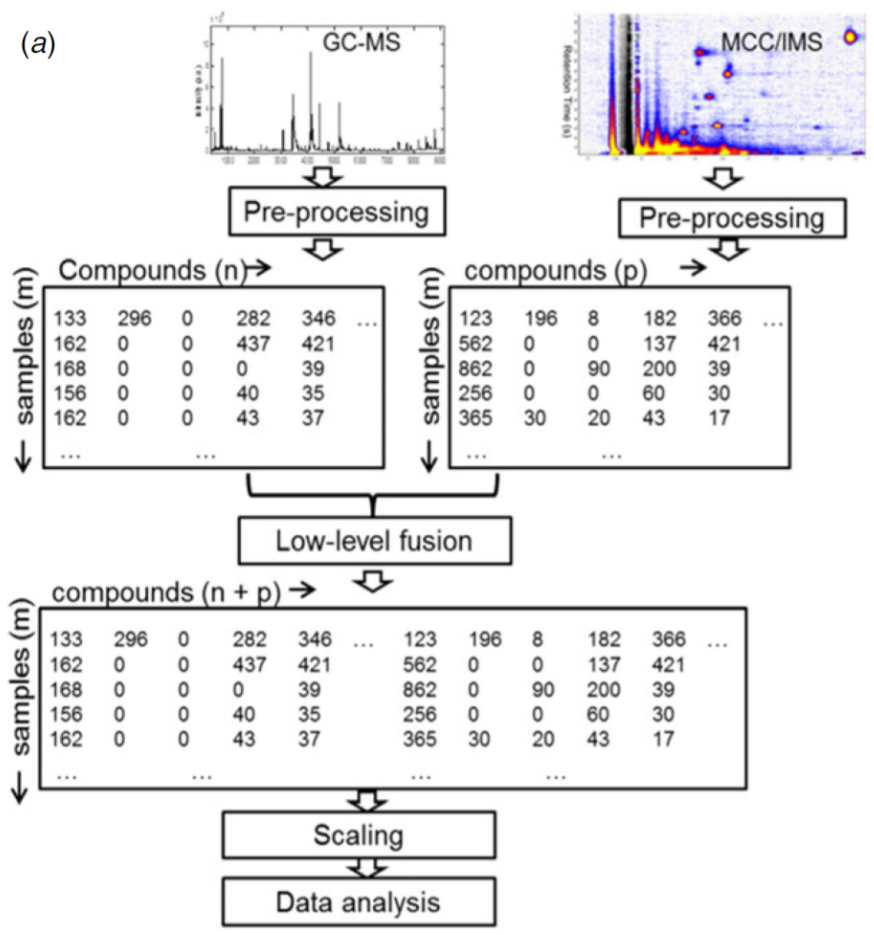

Figure 2.6: A schematic overview of various fusion approaches: (a) low-level data fusion; (b) mid-level data fusion and (c) high-level data fusion.

is to merge various information sources and use them to multiply the benefits of the multivariate approach (increasing performance and possible interpretation), not to take per se a retrospective decision as to which platform is the more relevant one. However, within MKL, it is possible to define which platform is the most important one. Clearly, the platform with the higher weight in the MKL procedure delivers the most optimal information about a studied problem (e.g. difference between disease and healthy groups).

\subsection{Validation of multivariate statistical models}

As was mentioned before, the validation of the outcomes of the supervised methods is a crucial part of data analysis. Proper validation of supervised methods is crucial to give certainty to the findings, i.e., the differences between the studied classes and the selected classifying compounds. Therefore, it is obligatory to check the model performance using various ways of assessing its predictive ability and many methods have been described [97, 122, 123]. In the ideal case (i.e., with a sufficient number of samples), the data are divided into a training set (used to train the classification method), a validation set (used to optimize the parameters) and an independent test 


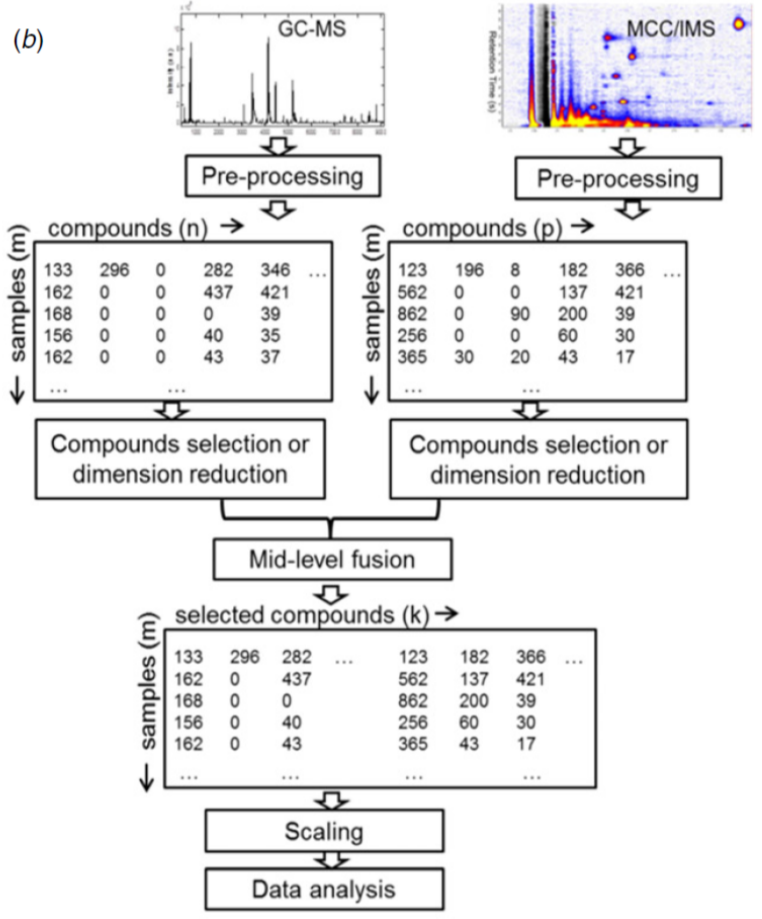

Figure 2.6: (Continued.)

set to assess the predictive power of the model. Within supervised analysis, the model is usually constructed using a training set and then verified with a test set. In the breathomics field, CV is the most commonly applied approach. A simple CV method is the leaving-one-out CV (LOO CV) in which the dataset is divided into as many folds as there are samples. In an iterative process, one sample is excluded from the data. The training set consists of $n 1$ samples, where ' $n$ ' is the total number of samples while a test set always consists of one sample. Each sample will become a test sample once. Therefore, there are ' $n$ ' rounds of testing as well. The error value is calculated at each iteration, and the final error is expressed as an average value. LOO CV is usually used when the number of available samples per class is low (around 20 per class). Alternatively, k-fold CV can be applied using a k of 5 or 10. This means that, in each CV fold, five or ten samples are removed from the training set and used as a test set. It has to be emphasized here that CV is far from being optimal to validate the prediction ability; the obtained error is too optimistic. Thus, it is advised that, if the number of samples is not sufficient (thus, it is not possible to divide into a training and test set), one should apply double CV (also called nested CV) or bootstrapping [122]. Another way of assessing the prediction ability of a classification model is to use a permutation test. It evaluates whether the division on two defined classes is significantly better than any random division [123]. In the permutation test, real class labels are randomly permuted and a classification 


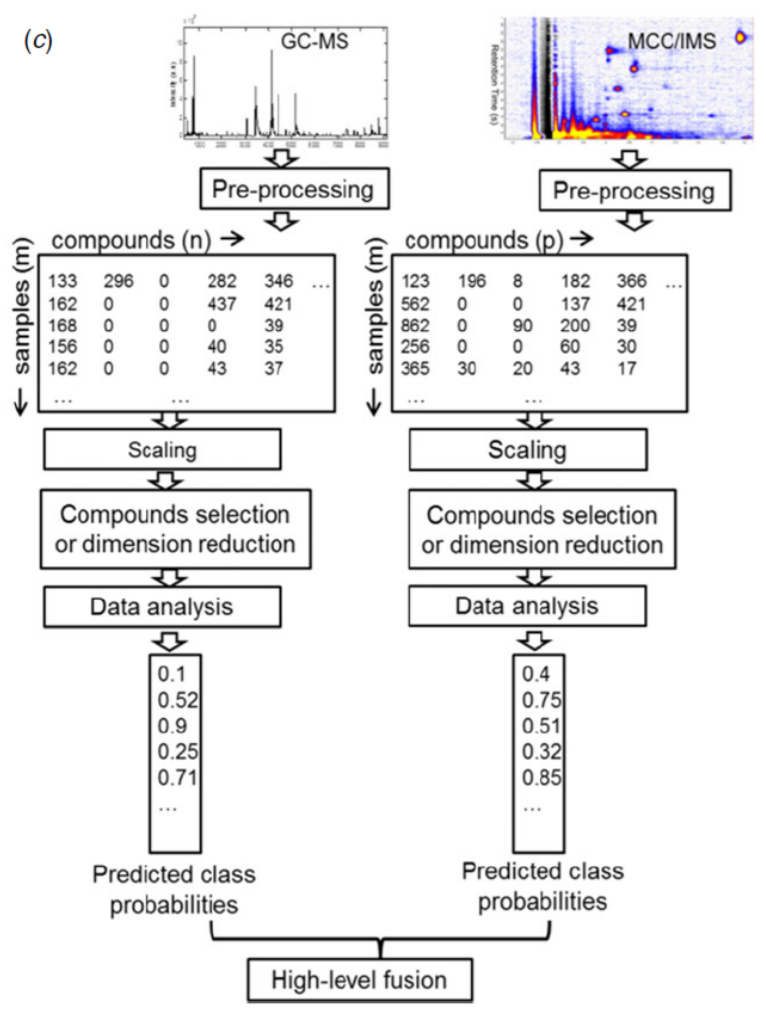

Figure 2.6: (Continued.)

model is built using these random classes. In the next step, the classification model is calculated for the false class labels and used to predict test samples. The procedure is repeated many times, and each time the NMC is obtained. Because the permuted classes are formed in a random way, the assumption is that the test classes should be wrongly predicted.

A more ideal situation for validation is when the data set is large enough to include an external test population. To obtain an external test set (sometimes called an independent test set), the available data are portioned into a training set and a test set. For splitting the data e.g. the Kennard and Stone algorithm [124], Duplex technique [125] or random selection are used. However, still the ultimate way of assessing the prediction ability of a statistical model is to use a set of completely new samples coming from a newly independent sampled population. This ultimate validation should be generally applied. 


\subsection{Summary}

In this paper, a complete overview of the main steps involved in data pre-processing and analysis of breathomics data are given. For the pre-processing part, the focus is mainly on data produced by GC-MS and MCC-IMS. We have described several ways of scaling and normalizing the breathomics data and given hints on how to highlight the information of interest. For data analysis, we have focused on different machine learning techniques. We described when they should be applied and what information they can bring. The multivariate techniques described here belong to well-established methods in the machine learning field, which are reasonably easy to apply and potentially insightful.

We believe that biomarker discovery by means of breathomics in combination with machine learning techniques is likely to make an increasing contribution in personalized medicine. 


\section{Bibliography}

[1] Silvia Carraro, Serge Rezzi, Fabiano Reniero, et al. Metabolomics applied to exhaled breath condensate in childhood asthma. Am. J. Respir. Crit. Care, 175(10):986, may 2007.

[2] Agnes W Boots, Joep J B N van Berkel, Jan W Dallinga, et al. The versatile use of exhaled volatile organic compounds in human health and disease. Journal of Breath Research, 6(2):27108, jun 2012.

[3] Wolfram Miekisch, Jochen K Schubert, and Gabriele F.E Noeldge-Schomburg. Diagnostic potential of breath analysisfocus on volatile organic compounds. Clinica Chimica Acta, 347(1):2539, sep 2004.

[4] Bogus?aw Bogusław Bogus?aw Buszewski, Martyna Kȩsy, Tomasz Ligor, et al. Human exhaled air analytics: biomarkers of diseases. Biomedical Chromatography, 21(6):553-566, jun 2007.

[5] Wolfram Miekisch, Jens Herbig, and Jochen K Schubert. Data interpretation in breath biomarker research: pitfalls and directions. J. Breath Res., 6(3):036007, sep 2012.

[6] Igor Kononenko. Machine learning for medical diagnosis: history, state of the art and perspective. Artif. Intell. Med., 23(1):89, aug 2001.

[7] Joachim D Pleil, Matthew A Stiegel, Jon R Sobus, Qian Liu, and Michael C Madden. Observing the human exposome as reflected in breath biomarkers: heat map data interpretation for environmental and intelligence research. J. Breath Res., 5(3):037104, sep 2011.

[8] Simon M. Scott, David James, and Zulfiqur Ali. Data analysis for electronic nose systems. Microchim. Acta, 156(3-4):183, dec 2006.

[9] Steven Goodman. A dirty dozen: twelve p-value misconceptions. Semin. Hematol., 45(3):135, jul 2008.

[10] James D Malley, Abhijit Dasgupta, and Jason H Moore. The limits of p-values for biological data mining. Biodata Min., 6:10, 2013.

[11] Krzanowski. Principles of Multivariate Analysis. Oxford University Press, 2000.

[12] Andrew R. Webb. Statistical Pattern Recognition. John Wiley \& Sons, Ltd, Chichester, UK, jul 2002.

[13] Vandeginste, Massart, Buydens, et al. Handbook of Chemometrics and Qualimerics: Part. Elsevier Science B.V, 1998.

[14] Tom M. (Tom Michael) Mitchell. Machine Learning. McGraw-Hill, 1997.

[15] J. Throck Watson and O. David Sparkman. Introduction to Mass Spectrometry. John Wiley \& Sons, Ltd, Chichester, UK, oct 2007. 
[16] Maren Mieth, Jochen K. Schubert, Thomas Groger, et al. Automated needle trap heart-cut GC/MS and needle trap comprehensive two-dimensional GC/TOF-MS for breath gas analysis in the clinical environment. Anal. Chem., 82(6):2541, mar 2010.

[17] J. J B N Van Berkel, J. W. Dallinga, G. M. Möller, et al. A profile of volatile organic compounds in breath discriminates COPD patients from controls. Respiratory Medicine, 104(4):557-563, apr 2010

[18] Michael Karas. ?Time-of-Flight Mass Spectrometer with Improved Resolution,? W. C. Wiley and I. H. McLaren,Rev.Sci.Instrum., 26, 1150 (1955). Journal of Mass Spectrometry, 32(1):111, jan 1997.

[19] Jörg Ingo Baumbach. Ion mobility spectrometry coupled with multi-capillary columns for metabolic profiling of human breath. J. Breath Res., 3(3):1, sep 2009.

[20] Vera Ruzsanyi, J?rg Ingo Baumbach, Stefanie Sielemann, et al. Detection of human metabolites using multi-capillary columns coupled to ion mobility spectrometers. J. Chromatogr., 1084(12):145, aug 2005.

[21] Sasidhar Maddula, Lars Mathias Blank, Andreas Schmid, and J??rg Ingo Baumbach. Detection of volatile metabolites of Escherichia coli by multi capillary column coupled ion mobility spectrometry. Analytical and Bioanalytical Chemistry, 394(3):791-800, jun 2009.

[22] J. I. Baumbach. Process analysis using ion mobility spectrometry. Anal. Bioanal. Chem., 384(5):1059, feb 2006.

[23] J. Trygg, J. Gabrielsson, and T. Lundstedt. 2.01 Background Estimation, Denoising, and Preprocessing. In Comprehensive Chemometrics, pages 1-8. 2009.

[24] Sonja Peters, Ewoud van Velzen, and Hans-Gerd Janssen. Parameter selection for peak alignment in chromatographic sample profiling: objective quality indicators and use of control samples. Analytical and Bioanalytical Chemistry, 394(5):1273, jul 2009.

[25] Arjen Lommen. MetAlign: interface-driven, versatile metabolomics tool for hyphenated fullscan mass spectrometry data preprocessing. Anal. Chem., 81(8):3079, apr 2009.

[26] Nils Hoffmann, Matthias Keck, Heiko Neuweger, et al. Combining peak- and chromatogrambased retention time alignment algorithms for multiple chromatography-mass spectrometry datasets. BMC Bioinformatics, 13(1):214, aug 2012.

[27] Tsung-Jung Ho, Ching-Hua Kuo, San-Yuan Wang, Guan-Yuan Chen, and Yufeng J. Tseng. True ion pick (TIPick): a denoising and peak picking algorithm to extract ion signals from liquid chromatography/mass spectrometry data. J. Mass Spectrom., 48(2):234, feb 2013.

[28] Leco ChromaTOF.

[29] Luca Cappellin, Franco Biasioli, Pablo M. Granitto, et al. On data analysis in PTR-TOF-MS: from raw spectra to data mining. Sensors Actuators, 155(1):183, jul 2011.

[30] M. Muller, M. Graus, T. M. Ruuskanen, et al. First eddy covariance flux measurements by PTR-TOF. Atmos. Meas. Tech., 3(2):387, mar 2010.

[31] Martin Graus, Markus Müller, and Armin Hansel. High resolution PTR-TOF: quantification and formula confirmation of VOC in real time. J. Am. Soc. Mass Spectrom., 21(6):1037, jun 2010.

[32] K.T. Moorhead, D. Lee, J.G. Chase, et al. Classifying algorithms for SIFT-MS technology and medical diagnosis. Comput. Methods Programs Biomed., 89(3):226, mar 2008.

[33] B. Walczak and D.L. Massart. Wavelets in Chemistry, volume 22. Elsevier Science B.V, nov 2000.

[34] G. E. P. Box and D. R. Cox. An analysis of transformations. Journal of the Royal Statistical Society. Series B (Methodological, pages 211-252, 1964.

[35] Olav M. Kvalheim, Frode. Brakstad, and Yizeng. Liang. Preprocessing of analytical profiles in the presence of homoscedastic or heteroscedastic noise. Anal. Chem., 66(1):43, jan 1994.

[36] Paul H. C. Eilers. A perfect smoother. Analytical Chemistry, 75(14):3631, jul 2003. 
[37] Paul H. C. Eilers and Brian D. Marx. Flexible smoothing with B -splines and penalties. Statistical Science, 11(2):89-121, may 1996.

[38] Zhanfeng Xu, Xiaobo Sun, and Peter de B. Harrington. Baseline correction method using an orthogonal basis for gas chromatography/mass spectrometry data. Anal. Chem., 83(19):7464, oct 2011 .

[39] Tom G. Bloemberg, Jan Gerretzen, Hans J.P. Wouters, et al. Improved parametric time warping for proteomics. Chemometr. Intell. Lab. Syst., 104(1):65, nov 2010.

[40] Niels-Peter Vest P V Nielsen, Jens Michael Carstensen, and J?rn Jørn Smedsgaard. Aligning of single and multiple wavelength chromatographic profiles for chemometric data analysis using correlation optimised warping. Journal of Chromatography A, 805(1-2):17-35, may 1998.

[41] Giorgio Tomasi, Frans van den Berg, and Claus Andersson. Correlation optimized warping and dynamic time warping as preprocessing methods for chromatographic data. J. Chemometr., 18(5):231, may 2004.

[42] Tomáš Pluskal, Sandra Castillo, Alejandro Villar-Briones, and Matej Orešič. MZmine 2: Modular framework for processing, visualizing, and analyzing mass spectrometry-based molecular profile data. BMC Bioinformatics, 11(1):395, jul 2010.

[43] Xiaoli Wei, Xue Shi, Mark Merrick, et al. A method of aligning peak lists generated by gas chromatography high-resolution mass spectrometry. Analyst, 138(18):5453, aug 2013.

[44] R.H. Jellema. 2.06 Variable Shift and Alignment. In Comprehensive Chemometrics, pages 85-108. 2009.

[45] Sabine Bader. Identification and quantification of peaks in spectrometric data. Technical University of Dortmund: Dortmund, Germany, 2008.

[46] Alexander Bunkowski. MCC-IMS data analysis using automated spectra processing and explorative visualisation methods. 2012.

[47] Sabine Bader, Wolfgang Urfer, and Jörg Ingo Baumbach. Reduction of ion mobility spectrometry data by clustering characteristic peak structures. J. Chemometr., 20(3-4):128, mar 2007.

[48] Dominik Kopczynski, J\{\"o\}rg Ingo Baumbach, and Sven Rahmann. Peak modeling for ion mobility spectrometry measurements. Proc. 20th European Signal Processing Conf., page 1801, 2012.

[49] Bertram Bödeker, Wolfgang Vautz, and Jörg Ingo Baumbach. Peak finding and referencing in MCC/IMSdata. Int. J. Ion Mobility Spectrom., 11(1-4):83, dec 2008.

[50] L. Vincent and P. Soille. Watersheds in digital spaces: an efficient algorithm based on immersion simulations. IEEE Trans. Pattern Anal. Mach. Intell., 13(6):583, jun 1991.

[51] Sim S. Fong, Preshious Rearden, Chitra Kanchagar, et al. Automated peak detection and matching algorithm for gas chromatography-differential mobility spectrometry. Anal. Chem., 83(5):1537, mar 2011.

[52] Frank Dieterle, Alfred Ross, Götz Schlotterbeck, et al. Probabilistic quotient normalization as robust method to account for dilution of complex biological mixtures. Application in1H NMR metabonomics. Analytical Chemistry, 78(13):4281-4290, jul 2006.

[53] R. J. O. Torgrip, K. M. ?berg, E. Alm, I. Schuppe-Koistinen, and J. Lindberg. A note on normalization of biofluid 1D 1H-NMR data. Metabolomics, 4(2):114-121, jun 2008.

[54] Jiyang Dong, Kian-Kai Cheng, Jingjing Xu, Zhong Chen, and Julian L. Griffin. Group aggregating normalization method for the preprocessing of NMR-based metabolomic data. Chemometr. Intell. Lab. Syst., 108(2):123, oct 2011.

[55] M. Bylesjö, O. Cloarec, and M. Rantalainen. 2.07 Normalization and Closure. In Comprehensive Chemometrics, pages 109-127. 2009.

[56] Hector C. Keun, Timothy M.D. Ebbels, Henrik Antti, et al. Improved analysis of multivariate data by variable stability scaling: application to NMR-based metabolic profiling. Anal. Chim. Acta, 490(1-2):265, aug 2003. 
[57] Robert A van den Berg, Huub CJ Hoefsloot, Johan A Westerhuis, Age K Smilde, and Mariët J van der Werf. Centering, scaling, and transformations: improving the biological information content of metabolomics data. BMC Genomics, 7:142, 2006.

[58] W J Conover and R L Iman. Rank transformations as a bridge between parametric and nonparametric statistics. Am. Stat., 35:124, 1981.

[59] David I. Broadhurst and Douglas B. Kell. Statistical strategies for avoiding false discoveries in metabolomics and related experiments. Metabolomics, 2(4):171-196, nov 2006.

[60] Jiangjiang Zhu, Heather D. Bean, Yin-Ming Ming Kuo, and Jane E. Hill. Fast detection of volatile organic compounds from bacterial cultures by secondary electrospray ionization-mass spectrometry. Journal of Clinical Microbiology, 48(12):4426-4431, dec 2010.

[61] Tomasz Dymerski, Jacek Gębicki, Paulina Wiśniewska, et al. Application of the electronic nose technique to differentiation between model mixtures with COPD markers. Sensors (Basel), 13(4):5008, apr 2013.

[62] Agnieszka Baranska, Ettje Tigchelaar, Agnieszka Smolinska, et al. Profile of volatile organic compounds in exhaled breath changes as a result of gluten-free diet. Journal of breath research, 7(3):037104, sep 2013.

[63] Helna Passinho-Soares, Paloma Meira, Juceni Jorge David, et al. Volatile organic compounds obtained by in vitro callus cultivation of plectranthus ornatus codd. (Lamiaceae). Molecules, 18(9):10320, aug 2013.

[64] G. de Gennaro, S. Dragonieri, F. Longobardi, et al. Chemical characterization of exhaled breath to differentiate between patients with malignant plueral mesothelioma from subjects with similar professional asbestos exposure. Anal. Bioanal. Chem., 398(7-8):3043, dec 2010.

[65] María Verónica Baroni, María Laura Nores, § María Del Pilar Díaz, et al. Determination of volatile organic compound patterns characteristic of five unifloral honey by solid-phase microextraction-gas chromatography-mass spectrometry coupled to chemometrics. J. Agric. Food Chem., 54:7235, 2006.

[66] Bo Li, Jinhuai Juefu Liu, Guolong Shi, and Jinhuai Juefu Liu. A research on detection and identification of volatile organic compounds utilizing cataluminescence-based sensor array. Sensor Actuators, 177:1167, feb 2013.

[67] Ritaban Dutta, K.R Kashwan, M Bhuyan, E.L Hines, and J.W Gardner. Electronic nose based tea quality standardization. Neural Netw., 16(5):847, 2003.

[68] M. Statheropoulos, K. Mikedi, A. Agapiou, A. Georgiadou, and S. Karma. Discriminant analysis of volatile organic compounds data related to a new location method of entrapped people in collapsed buildings of an earthquake. Anal. Chim. Acta, 566(2):207, may 2006.

[69] J. W. Dallinga, C. M H H T Robroeks, J. J B N Van Berkel, et al. Volatile organic compounds in exhaled breath as a diagnostic tool for asthma in children. Clinical and Experimental Allergy, 40(1):68-76, jan 2010.

[70] S M Cristescu, H A Gietema, L Blanchet, et al. Screening for emphysema via exhaled volatile organic compounds. Journal of Breath Research, 5(4):046009, dec 2011.

[71] M. Caldeira, A.S. S Barros, M.J. J Bilelo, et al. Profiling allergic asthma volatile metabolic patterns using a headspace-solid phase microextraction/gas chromatography based methodology. J. Chromatogr., 1218(24):3771, jun 2011.

[72] Li-eng Xu, Yan-qing Feng, and Jian-qi Chen. [Study on simultaneous analysis of indoor air multi-component VOCs with FTIR]. Guang pu xue yu guang pu fen $x i=G u a n g ~ p u$, 26(12):2197-9, dec 2006.

[73] P. J Mazzone, J. Hammel, R. Dweik, et al. Diagnosis of lung cancer by the analysis of exhaled breath with a colorimetric sensor array. Thorax, 62(7):565-568, feb 2007.

[74] Bravein Amalakuhan, Lukasz Kiljanek, Arvin Parvathaneni, et al. A prediction model for COPD readmissions: catching up, catching our breath, and improving a national problem. Journal of community hospital internal medicine perspectives, 2(1), 2012. 
[75] J.J.B.N. Van Berkel, J.W. Dallinga, G.M. Möller, et al. Development of accurate classification method based on the analysis of volatile organic compounds from human exhaled air. Journal of Chromatography B, 861(B, 861):101-107, jan 2008.

[76] Feng Luan, Chunxia Xue, Ruisheng Zhang, et al. Prediction of retention time of a variety of volatile organic compounds based on the heuristic method and support vector machine. Anal. Chim. Acta, 537(1-2):101, apr 2005.

[77] D. F. Altomare, M. Di Lena, F. Porcelli, et al. Exhaled volatile organic compounds identify patients with colorectal cancer. British Journal of Surgery, 100(1):144-150, jan 2013.

[78] Changjun Hou, Jincan Lei, Danqun Huo, et al. Discrimination of lung cancer related volatile organic compounds with a colorimetric sensor array. Anal. Lett., 46(13):2048, sep 2013.

[79] Trevor Hastie, Robert Tibshirani, and J. H. (Jerome H.) Friedman. The elements of statistical learning : data mining, inference, and prediction.

[80] Michal Daszykowski. From projection pursuit to other unsupervised chemometric techniques. J. Chemometr., 21(7-9):270, jul 2007.

[81] M. Daszykowski, K. Kaczmarek, Y. Vander Heyden, and B. Walczak. Robust statistics in data analysis ? A review. Chemometrics and Intelligent Laboratory Systems, 85(2):203-219, feb 2007.

[82] Rasmus Bro, Evangelos E. Papalexakis, Evrim Acar, and Nicholas D. Sidiropoulos. Coclusteringa useful tool for chemometrics. J. Chemometr., 26(6):256, jun 2012.

[83] S.C. Madeira and A.L. Oliveira. Biclustering algorithms for biological data analysis: a survey. IEEE/ACM Trans. Comput. Biol. Bioinformatics, 1(1):24, jan 2004.

[84] L Blanchet. Discrimination between metastasis and glioblastoma multiforme based on morphometric analysis of MR images. AJNR Am. J. Neuroradiol., 32:67, 2011.

[85] Ulrike von Luxburg. A tutorial on spectral clustering. Stat. Comput., 17(4):395, dec 2007.

[86] Tobias Wittkop, Dorothea Emig, Sita Lange, et al. Partitioning biological data with transitivity clustering. Nature Methods, 7(6):419, jun 2010.

[87] Guglielmo de Laurentiis, Debora Paris, Dominique Melck, et al. Separating smoking-related diseases using NMR-based metabolomics of exhaled breath condensate. J. Proteome Res., 12(3):1502, mar 2013.

[88] Niki Fens, Annelot G.J. van Rossum, Pieter Zanen, et al. Subphenotypes of mild-to-moderate COPD by factor and cluster analysis of pulmonary function. COPD, 10(3):277, jun 2013.

[89] S. Chatterjee, M. Castro, J. F. Feller, et al. An e-nose made of carbon nanotube based quantum resistive sensors for the detection of eighteen polar/nonpolar VOC biomarkers of lung cancer. J. Mater. Chem., 1(36):4563, aug 2013.

[90] Silvano Dragonieri, Paul Brinkman, Evert Mouw, et al. An electronic nose discriminates exhaled breath of patients with untreated pulmonary sarcoidosis from controls. Respir. Med., 107(7):1073, jul 2013.

[91] Phillip H. Rogers, Kurt D. Benkstein, and Steve Semancik. Machine learning applied to chemical analysis: sensing multiple biomarkers in simulated breath using a temperature-pulsed electronic-nose. Anal. Chem., 84(22):9774, nov 2012.

[92] Jesica Dadamio, Sandra Van den Velde, Wim Laleman, et al. Breath biomarkers of liver cirrhosis. J. Chromatogr., 905:17, sep 2012.

[93] Froukje J. Verdam, Jan W. Dallinga, Ann Driessen, et al. Non-alcoholic steatohepatitis: a non-invasive diagnosis by analysis of exhaled breath. J. Hepatol., 58(3):543-548, mar 2013.

[94] Richard O. Duda, Peter E. (Peter Elliot) Hart, and David G. Stork. Pattern classification. Wiley, 2001.

[95] Lars Nørgaard, Rasmus Bro, Frank Westad, and S?ren Balling Engelsen. A modification of canonical variates analysis to handle highly collinear multivariate data. J. Chemometr., 20(810):425, aug 2006 . 
[96] Svante Wold, Michael Sjöström, and Lennart Eriksson. PLS-regression: A basic tool of chemometrics. Chemometrics and Intelligent Laboratory Systems, 58(2):109-130, oct 2001.

[97] Ewa Szymańska, Edoardo Saccenti, Age K. Smilde, and Johan A. Westerhuis. Double-check: Validation of diagnostic statistics for PLS-DA models in metabolomics studies. Metabolomics, 8(Suppl 1):3-16, jun 2012.

[98] Il-Gyo Chong and Chi-Hyuck Jun. Performance of some variable selection methods when multicollinearity is present. Chemometr. Intell. Lab. Syst., 78(1-2):103, jul 2005.

[99] Tarja Rajalahti, Reidar Arneberg, Ann C. Kroksveen, et al. Discriminating variable test and selectivity ratio plot: quantitative tools for interpretation and variable (biomarker) selection in complex spectral or chromatographic profiles. Anal. Chem., 81(7):2581, apr 2009.

[100] Pieter C Goeminne, Thomas Vandendriessche, Johan Van Eldere, et al. Detection of Pseudomonas aeruginosa in sputum headspace through volatile organic compound analysis. Respiratory Research, 13(1):87, oct 2012.

[101] Johan Trygg and Svante Wold. Orthogonal projections to latent structures (O-PLS). Journal of Chemometrics, 16(3):119-128, mar 2002.

[102] Isabelle A Guyon. An introduction to variable and feature selection. J. Mach. Learn. Res., 3:1157, 2003.

[103] Agnieszka Smolinska, Lionel Blanchet, Leon Coulier, et al. Interpretation and visualization of non-linear data fusion in kernel space: study on metabolomic characterization of progression of multiple sclerosis. Plos One, 7(6):e38163, jun 2012.

[104] D. Coomans and D.L. Massart. Alternative k-nearest neighbour rules in supervised pattern recognition. Analytica Chimica Acta, 136:15-27, 1982.

[105] Leo. Breiman. Classification and regression trees. Chapman \& Hall, 1993.

[106] Leo Breiman. Random forests. Machine Learning, 45(1):5-32, oct 2001.

[107] M. Westhoff, P. Litterst, S. Maddula, et al. Statistical and bioinformatical methods to differentiate chronic obstructive pulmonary disease (COPD) including lung cancer from healthy control by breath analysis using ion mobility spectrometr. Int. J. Ion Mobility Spectrom., 11(4):139, dec 2011.

[108] A.-C. Hauschild, J.I. I Baumbach, and J.I. I Baumbach. Integrated statistical learning of metabolic ion mobility spectrometry profiles for pulmonary disease identification. Genetics and Molecular Research, 11(3):2733, aug 2012.

[109] Miao Liu, Mingjun Wang, Jun Wang, and Duo Li. Comparison of random forest, support vector machine and back propagation neural network for electronic tongue data classification: application to the recognition of orange beverage and chinese vinegar. Sensors Actuators, 133:970, feb 2013.

[110] Jörg Ingo Jan Baumbach, Alexander Bunkowski, Sita Lange, et al. IMS2 - An integrated medical software system for early lung cancer detection using ion mobility spectrometry data of human breath. J. Integr. Bioinformatics, 4(3):186-197, jan 2007.

[111] Patrick W. T. Krooshof, Bulent Ustun, Geert J. Postma, et al. Visualization and recovery of the (bio)chemical interesting variables in data analysis with support vector machine classification. Anal. Chem., 82(16):7000, aug 2010.

[112] Isabelle Guyon, Jason Weston, Stephen Barnhill, and Vladimir Vapnik. Gene selection for cancer classification using support vector machine. Mach. Learn., 46(1/3):389, 2002.

[113] Filippo Amato, Alberto Lopez, Eladia Maria Pena-Mendez, et al. Artificial neural networks in medical diagnosis. J. Appl. Biomed., 11(2):47, 2013.

[114] Hamdi Melih Saraoglu and Mehmet Kocan. Determination of blood glucose level-based breath analysis by a quartz crystal microbalance sensor array. IEEE Sensors J., 10(1):104, jan 2010.

[115] M Phillips, V Basa-Dalay, J Blais, et al. Point-of-care breath test for biomarkers of active pulmonary tuberculosis. Tuberculosis, 92, 2012. 
[116] Rossella Blatt, Andrea Bonarini, and Matteo Matteucci. Pattern classification techniques for lung cancer diagnosis by an electronic nose. Computational Intelligence in Healthcare 4: Advanced Methodologies, 309:397, 2010.

[117] D.L. Hall and J. Llinas. An introduction to multisensor data fusion. Proc. IEEE, 85(1):6, 1997.

[118] Ludmila I. Kuncheva. Combining Pattern Classifiers. John Wiley \& Sons, Inc., Hoboken, NJ, USA, jul 2004.

[119] David L. (David Lee) Hall. Mathematical techniques in multi-sensor data fusion. Artech House, 2004.

[120] Gert R.G. Lanckriet, Nello Cristianini, Peter Bartlett, Laurent El Ghaoui, and Michael I. Jordan. Learning the Kernel Matrix with Semidefinite Programming. Journal of Machine Learning Research, 5(Jan):27, 2004.

[121] Francis R. Bach. Consistency of the Group Lasso and Multiple Kernel Learning. Journal of Machine Learning Research, 9(Jun):1179-1225, 2008.

[122] Endre Anderssen, Knut Dyrstad, Frank Westad, and Harald Martens. Reducing over-optimism in variable selection by cross-model validation. Chemometrics and Intelligent Laboratory Systems, 84(1-2 SPEC. ISS.):69-74, dec 2006.

[123] Johan A. Westerhuis, Huub C. J. Hoefsloot, Suzanne Smit, et al. Assessment of PLSDA cross validation. Metabolomics, 4(1):81, mar 2008.

[124] R. W. Kennard and L. A. Stone. Computer aided design of experiments. Technometrics, 11(1):137, feb 1969.

[125] Ronald D. Snee. Validation of regression models: methods and examples. Technometrics, 19(4):415, nov 1977. 



\section{CHAPTER 3}

Analysis of volatile organic compounds in exhaled breath to diagnose ventilator-associated pneumonia

$R$ Fijten, $R$ Schnabel, A Smolinska, J Dallinga, M Boumans, E Stobberingh, A Boots, P Roekaerts, D Bergmans, F van Schooten

Sci Rep. 2015 Jan 26;5:17179 


\subsection{Abstract}

Ventilator-associated pneumonia (VAP) is a nosocomial infection occurring in the intensive care unit (ICU). The diagnostic standard is based on clinical criteria and bronchoalveolar lavage (BAL). Exhaled breath analysis is promising as a non-invasive method for rapid diagnosis of diseases. Breath contains volatile organic compounds (VOCs) that can differentiate diseased from healthy individuals. The aim of this study was to determine whether analysis of VOCs in exhaled breath can be used as a non-invasive monitoring tool for VAP.

One hundred critically ill patients with clinical suspicion of VAP underwent BAL. Before BAL, exhaled air samples were collected and analysed by gas chromatography time-of-flight mass spectrometry (GC-tof-MS). The clinical suspicion of VAP was confirmed by BAL diagnostic criteria in 32 patients $[\operatorname{VAP}(+)]$ and rejected in 68 patients $[\operatorname{VAP}(-)]$. Statistical comparison of $\operatorname{VOC}$ profiles between $\operatorname{VAP}(+)$ and VAP(-) was performed using multivariate statistics and revealed that a subset of 12 VOCs correctly discriminated between those two patient groups with a sensitivity and specificity of $75.8 \%+-13.5 \%$ and $73.0 \%+-11.8 \%$, respectively.

These results suggest that detection of VAP in ICU patients is possible examining exhaled breath, enabling a simple, safe and non-invasive approach that could diminish diagnostic burden of VAP.

\subsection{Introduction}

Ventilator-associated pneumonia (VAP) is a common hospital-acquired infection occurring in the intensive care unit (ICU) with an incidence that varies from $4 \quad 42 \%$ depending on the applied diagnostic criteria [1]. It is a severe complication of mechanical ventilation with an attributable mortality risk of approximately $13 \%$ [2]. To date, the diagnosis is based on clinical criteria in combination with bacterial culture results. In patients clinically suspected of having VAP, bronchoalveolar lavage (BAL) from the site of the presumed infection and subsequently cytological and microbiological analysis of the lavage fluid is regarded a specific diagnostic approach [3]. However, this technique is invasive, involves risks and has its limitations in patients with severe pulmonary disease, high respiratory support settings and coagulation abnormalities. Additionally, analysis of BAL is laborious, time- consuming and takes up to 48 hours before definitive results are available. Only then, the diagnosis of VAP can be confirmed or rejected. During this period patients empirically receive broad spectrum antibiotics. Facing a rapid emergence and dissemination of multi-drug resistant microorganisms particularly in the ICU environment, strategies to reduce such general and non-targeted antibiotic consumption become very important [4].

It is therefore of interest to find a new method that allows fast, reliable, noninvasive VAP diagnosis. Using exhaled breath for disease diagnosis is a promising technique that may be able to fulfil these criteria. Exhaled breath contains a multitude of volatile organic compounds (VOCs) originating from both exogenous and endogenous sources. Endogenous VOCs are produced by biological processes including oxidative stress and inflammation in the human body $[5,6]$ as well as by invading 
microorganisms [7]. Upon their production, VOCs are excreted into the blood after which they diffuse into the lungs where they are exhaled. Infectious diseases, such as pneumonia, involve oxidative stress and inflammation. These processes induce alterations in the composition of VOCs excreted by the affected organ and thus the exhaled breath. Additionally, microorganisms themselves may produce specific compounds leading to different VOC profiles in exhaled breath. Taking into account the invasion of harmful microorganisms in the lungs and the defence mechanisms that are subsequently set in motion by the host, it can be expected that VOCs are present in different concentrations and compositions in patients with VAP compared to patients without VAP. These discriminating VOC profiles may be used to aid VAP diagnosis.

Thus far, discriminating VOC profiles have been found for various respiratory diseases such as chronic obstructive pulmonary disease (COPD), asthma, tuberculosis and cystic fibrosis [8-13]. It has already been demonstrated that Pseudomonas aeruginosa, Staphylococcus aureus, Escherichia coli and Klebsiella pneumoniae could be identified correctly based on the analysis of VOCs excreted into the headspace of cultured bacteria [14]. Many of these strains frequently cause VAP. In another study, VOCs of Streptococcus pneumoniae and Haemophilus influenzae cultures were analysed at different time points during cultivation, leading to the identification of strain-specific VOCs for both bacterial species [15]. A systematic review summarized both strain-specific and commonly occurring VOCs from 31 recent in vitro studies that investigated bacterial species [7]. A recently published study by Fowler et al. found that, in a well-characterized group of patients with sterile brain injury, exhaled breath analysis is an effective method to detect the presence of airway pathogens in vivo that can induce VAP [16]. The aim of the current study is to identify $\operatorname{VAP}(-)$ specific VOCs in vivo by analysis of the exhaled breath of critically ill mechanically ventilated patients independent of their underlying disease on admission to the ICU.

\subsection{Materials and Methods}

\subsubsection{Study Design}

This study was conducted at Maastricht University Medical Centre+, a tertiary, university hospital in the Netherlands with 1,700 ICU admissions per year. The ICU consists of two 9- bed units for medical and surgical patients and one 9-bed unit for cardiothoracic surgery patients. Adult critically ill, mechanically ventilated patients with a clinical suspicion of VAP who underwent a diagnostic BAL were included. Exclusion criteria for the BAL procedure were thrombocytopenia $(\leq 40,000 / \mu L)$ and other coagulation abnormalities. The exhaled breath study and its experimental protocols were evaluated by the joint medical ethics committee at Maastricht University and Maastricht University Medical Centre+ (METC azM/UM). After evaluation and approval of the experimental protocols, the METC azM/UM committee concluded that the study did not fall under the scope of the medical research involving human subjects act (WMO), and was therefore denoted as non-WMO research as no direct and invasive patient intervention was required and results of the analyses did not influence the patients outcome. Experimental protocols were performed in accordance with the approved national Dutch guidelines for non-WMO research [17]. 
A patient was clinically suspected of VAP after $>48$ hours of mechanical ventilation, fulfilling the following clinical criteria:

- Rectal temperature above $38^{\circ} \mathrm{C}$ or below $35.5^{\circ} \mathrm{C}$

- An abnormal increase $(>10,000 / \mu \mathrm{l})$ or decrease $(<3,000 / \mu \mathrm{l})$ of leukocytes levels

- More than 10 leukocytes found in the tracheal aspirate

- Positive bacterial culture of the tracheal aspirate

In those patients, BAL was performed on the day of clinical suspicion for VAP. A fibreoptic bronchoscope (Pentax FB-15H/FB-15X, Pentax Medicals, Tokyo, Japan) was introduced and wedged into the affected segmental or subsegmental bronchus. Sterile saline ( $0.9 \%$ sodium chloride at room temperature) was instilled in four aliquots of $50 \mathrm{~mL}$, immediately aspirated and recovered. Further analysis was highly standardized as described by de Brauwer et al. [18]. A clinically suspected episode was considered microbiologically confirmed when the following criteria were met in BAL fluid (BALF): presence of $\geq 2 \%$ cells containing intracellular organisms (ICO) and/ or quantitative culture results of $\geq 104 c f u / m L[19,20]$. One hundred patients were included in the study. Upon BALF analysis they were divided into two groups: (1) BALF confirmed the clinical suspicion of $\operatorname{VAP}(\operatorname{VAP}(+), \mathrm{n}=32) ;(2)$ the diagnosis of VAP was rejected by BALF analysis $(\operatorname{VAP}(-), n=68)$. The Sequential Organ Failure Assessment (SOFA) score was registered at the moment of BAL to compare the seriousness of illness. The diagnosis of the underlying disease on admission to the ICU of all patients were documented and allocated into seven diagnostic groups. Differences between $\operatorname{VAP}(+)$ and $\operatorname{VAP}(-)$ were tested for significance: two-sided paired t-test for age and SOFA scores; chi square for diagnosis upon admission. A p-value $<0.05$ was considered significant. (Table 3.1)

\subsubsection{Sampling and measurement of exhaled breath}

Directly before BAL was performed, exhaled breath samples from ventilated patients were collected into a sterile Tedlar bag $(5 \mathrm{~L})$. The bag was tightly connected to the expiratory limb of the Draeger Evita XL ventilator (Lbeck, Germany). Exhaled breath from the patient could then flow into the Tedlar bag without any pollution from the environment. When the bag was filled, its valve was closed and the connection with the ventilator subsequently removed. The content of the bag was transported by vacuum pump (VWR International, France) onto stainless steel two-bed desorption tubes, filled with carbograph 1TD/Carbopack X (Markes International, Llantrisant, Wales, UK) that trap VOCs. The VOCs in these desorption tubes were measured by gas chromatography-time of flight-mass spectrometry (GC-tof-MS) based on the procedure described by Van Berkel et al. [8]. This was done in a non-targeted way, meaning that the highest amount and variety of VOCs were measured and used for multivariate statistical analysis later on. 
Table 3.1: Characteristics of the patient groups in the study. The age and SOFA scores were tested for significance with a two-sided paired t-test; significance was tested for the diagnostic groups using a Chi Square test. $\mathrm{P}<0.05$ was considered significant. Age is represented as mean standard deviation.

\begin{tabular}{|c|c|c|c|}
\hline Characteristics & $\operatorname{VAP}(+)$ & $\operatorname{VAP}(-)$ & p-value \\
\hline Sample size & 32 & 68 & \\
\hline Average age [years] & $64 \pm 12$ & $60 \pm 14.5$ & 0.16 \\
\hline Male/Female & $26 / 6$ & $44 / 24$ & 0.07 \\
\hline SOFA at time of BAL & $6.4 \pm 3.4$ & $6.9 \pm 2.9$ & 0.41 \\
\hline Diagnostic group at admission & & & 0.24 \\
\hline gastrointestinal & 4 & 9 & \\
\hline cardiovascular & 9 & 13 & \\
\hline hematologic & 3 & 15 & \\
\hline neurologic & 4 & 6 & \\
\hline orthopaedic/trauma & 4 & 2 & \\
\hline respiratory & 8 & 20 & \\
\hline other & 0 & 3 & \\
\hline \multicolumn{4}{|l|}{ Comorbidities } \\
\hline No comorbidity & $17(53 \%)$ & $38(56 \%)$ & 0.80 \\
\hline One comorbidity & $8(25 \%)$ & $15(22 \%)$ & \\
\hline Two comorbidities & $5(16 \%)$ & $12(18 \%)$ & \\
\hline$\geq$ three comorbidities & $2(6 \%)$ & $3(4 \%)$ & \\
\hline Distribution of comorbidities & & & 0.23 \\
\hline cardiovascular & 3 & 3 & \\
\hline respiratory & 3 & 5 & \\
\hline chronic renal failure & 4 & 7 & \\
\hline active malignancy & 2 & 9 & \\
\hline immunocompromised & 7 & 15 & \\
\hline neurologic impairment & 3 & 8 & \\
\hline chronic liver failure & 2 & 2 & \\
\hline Severe sepsis & $11(34 \%)$ & $24(35 \%)$ & 0.93 \\
\hline ICU mortality & $12(38 \%)$ & $31(45 \%)$ & 0.45 \\
\hline In hospital mortality & $14(43 \%)$ & $37(54 \%)$ & 0.34 \\
\hline
\end{tabular}

\subsubsection{Data processing and statistical analysis}

Raw GC-tof-MS data were pre-processed to remove various sources of artefacts before the actual statistical analysis. Pre-processing of the data reduces the influence of these artefacts and allows for the biological variation to come through. This was done by sequential use of the following methods: denoising, baseline correction, alignment, normalization and scaling of the data [21]. In order to compare different groups, the number of samples in the larger group has to be reduced to the size of the smaller group to make the statistical analyses work efficiently. This was done by randomly choosing a subset of 32 samples of the $68 \mathrm{VAP}(-)$ samples to match the size of the $\operatorname{VAP}(+)$ group and using this subset for further statistical analysis. This procedure 
was repeated 250 times to ensure that each sample was used. For this study, the multivariate statistical analysis method Random Forest (RF) was used [22].

This machine learning method constructs a multitude of de-correlated decision trees to classify samples into the appropriate disease state. Decision trees are predictive models that try to classify samples based on a specific subset of the measured VOCs. RF creates many decision trees (e.g. 1,000) comprising of a small and randomly selected subset of VOCs and tries to predict the class outcome. The most discriminatory subset of VOCs is then used to create the final classification model.

Validation of the RF model was done by calculating the out-of-bag error. In this procedure $66.7 \%$ of the samples are randomly selected with replacement for each decision tree. The remaining $33.3 \%$ are used to calculate the performance of the RF classification model. This produces class probability values, which are used to calculate sensitivity and specificity illustrated by Receiver Operating Characteristic (ROC) curves. For the sensitivity and specificity parameters, the $95 \%$ confidence interval was calculated and written in the following way: mean \pm confidence interval.

An ROC curve is a graphical representation of the performance of the predictive model established by RF. The area under the curve (AUC) is most commonly used as an indicator of predictive performance: a value close to 1 indicates high predictive power of the model, whereas an AUC close to 0.5 means that the model has no predictive power [23].

For visualization purposes, principal component analysis (PCA) score plots of the $\mathrm{RF}$ proximities were created. The proximities are distance parameters ranging from 0 to 1 that visualize similarities of the selected VOC profile between individual samples. A small proximity value indicates similarity, while a large proximity value indicates dissimilarity between individuals. A PCA plot of proximities can therefore demonstrate groupings of samples and trends in the data.

\subsubsection{Influence of confounders}

In order to rule out that the VOC profile found by RF is influenced by confounding factors, regularized MANOVA was used [24] to test the following possible confounders: age, gender, diagnostic group at admission, SOFA scores, ICU mortality, general hospital mortality, the presence of comorbidities in general, and the presence of specific comorbidities mentioned in Table 3.1.

\subsubsection{Compound identification}

The VOCs implemented into the classification model were identified with spectrum recognition using the National Institute of Standard and Technology (NIST) library in combination with spectrum interpretation by an experienced mass spectrometrist and identification based on retention times of components.

\subsubsection{Pathway identification}

For each of the chemically identified VOCs the ChEBI [25], ChemSpider [26] and PubChem IDs [27] were found in their respective databases. BridgeDb [28], which 
links IDs from several databases, was used to find additional IDs corresponding to each VOC. This was necessary due to annotation problems in pathways, where the same metabolite is mentioned with a differen ID or name in different pathways.

The RRDF package [29] was then used to find pathways in the Wikipathways [30] that included that specific VOC. Additionally, because Wikipathways includes only a limited number of metabolite pathways, the KEGG database [31] was analysed for pathways containing the identified VOCs. This was done using the KEGG REST api.

Because VAP is mainly caused by a well-defined array of bacteria, a selection of pathways was made that were present in the human host or in bacteria most likely to cause VAP [32] including Staphylococcus aureus, Pseudomonas aeruginosa, Escherichia coli, Klebsiella pneumoniae, Hemophilus influenzae.

\subsection{Results}

\subsubsection{Clinical outcome/data}

An overview of demographic and clinical data is presented in Table 3.1. Sample size varies between the groups as a result of confirmation of VAP in only 32 of 100 individuals included in the study. There were no significant differences in the seriousness of disease at the moment of BAL (SOFA) or in the distribution of the underlying diagnosis on admission to the ICU. However, patients in the $\operatorname{VAP}(-)$ group seemed to suffer more from a haematological diagnosis upon admission and active malignancies. (Table 3.1) During the study period we found an average of 2.5 episodes of VAP per 1,000 ventilator days. The diagnosis of VAP was based in 6 patients on a percentage of ICO $>2 \%$ alone and in 26 patients on a bacterial growth of more than $104 \mathrm{cfu} / \mathrm{mL}$. Staphylococcus aureus $(\mathrm{n}=5)$, Pseudomonas aeruginosa $(\mathrm{n}=4)$, Escherichia coli $(\mathrm{n}=4)$, Klebsiella pneumoniae $(\mathrm{n}=4)$, Hemophilus influenzae $(\mathrm{n}=3)$ and Acinetobacter baumannii $(\mathrm{n}=3)$ were the most frequently found microorganisms.

\subsubsection{RF classification model}

GC-MS measurements produced 100 chromatograms: one for each patient. After processing, these chromatograms consisted of ¿1000 chemically different VOCs. RF was used to filter $\operatorname{VOCs}$ that were discriminatory between $\operatorname{VAP}(+)$ and $\operatorname{VAP}(-)$. The final RF classification model was based on 12 discriminatory VOCs and correctly classified $74.2 \% \pm 13.8 \%$ of all individuals with a sensitivity and specificity of $75.8 \%$ $\pm 13.5 \%$ and $73.0 \% \pm 11.8 \%$ respectively. The corresponding ROC curve depicted in Figure 3.1A had an AUC of 0.87. The PCA score plot of proximities between the individual samples based on the 12 most important VOCs (Figure 3.1B) showed that the $\operatorname{VAP}(+)$ and $\operatorname{VAP}(-)$ patients are separated with small overlap. This indicates that patients suffering from VAP can be identified based on this combination of 12 VOCs with high accuracy. 

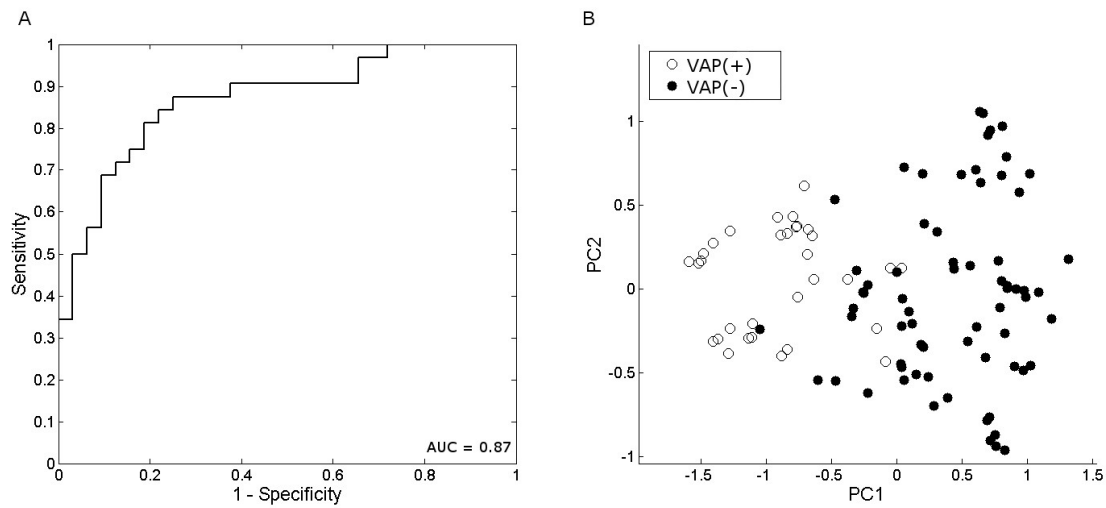

Figure 3.1: ROC and PCA plots visualizing the separation of the $\operatorname{VAP}(+)$ and VAP(-) groups. A: Receiver operating characteristic (ROC) curve of $\operatorname{VAP}(+)$ vs. $\operatorname{VAP}(-)$. It consists of 1-sensitivity on the $\mathrm{x}$-axis and specificity on the $\mathrm{y}$-axis. B: The PCA plot is based on the proximities between samples of $\operatorname{VAP}(+)$ (white) and $\operatorname{VAP}(-)$ (black).

\subsubsection{Influence of confounders}

The influence of potential confounders was tested to ensure that the discriminating VOC profile was purely a result of the VAP diagnosis. The following confounding factors were tested: age, gender, diagnostic group at admission, SOFA scores, ICU mortality, general hospital mortality, the presence of comorbidities in general, and the presence of specific comorbidities mentioned in Table 3.1 None of these confounders significantly influenced the model (Supplementary Table 3.S1).

\subsubsection{Compound identification}

The 12 VOCs selected by RF were chemically classified and their chemical identity is shown in Table 3.2. The identified VOCs include 2-methylbutane, heptane, dodecane and tetradecane (alkanes), 3,7,7-Trimethylbicyclo [4.1.0] heptane (hydrocarbon ring structure), ethanol and 2-propanol (alcohols), 2-propenal and tetradecanal (aldehydes). The remaining compounds were identified as acetone (ketone), ethylbenzene (aromatic hydrocarbon) and tetrahydrofuran (oxygen-containing heterocyclic compound).

\subsubsection{Pathway identification}

The Wikipathways and KEGG databases were each searched for pathways containing one or more of the 12 discriminatory VOCs and s. Only pathways present in humans and VAP-causing bacteria were included in the analysis. Seven KEGG pathways remained containing two VOCs (Table 3.3): one pathway that produced 2-propenal (Acrolein); five pathways with ethanol as an end-product and one which utilized 
Table 3.2: Identified VOCs for the comparison between $\operatorname{VAP}(+)$ and $\operatorname{VAP}(-)$. In addition to the compound name, CAS numbers were added for identification. Up/Down in $\operatorname{VAP}(+)$ vs. $\operatorname{VAP}(-)$ was based on mean peak height.

\begin{tabular}{|c|c|c|c|c|c|}
\hline Compound name & CAS nr & $\begin{array}{l}\text { Mole- } \\
\text { cular } \\
\text { formula }\end{array}$ & $\begin{array}{l}\mathrm{M} / \mathrm{z} \text { of } \\
\text { parent } \\
\text { molecule } \\
\text { (g mol- } \\
1)\end{array}$ & $\begin{array}{l}\text { Average } \\
\text { reten- } \\
\text { tion } \\
\text { time } \\
(\mathrm{min})\end{array}$ & $\begin{array}{l}\text { Up/Down } \\
\text { in } \\
\operatorname{VAP}(+) \\
\text { vs } \\
\operatorname{VAP}(-)\end{array}$ \\
\hline Butane , 2-methyl & $78-78-4$ & $\mathrm{C} 5 \mathrm{H} 12$ & 72.10 & 2.28 & $\uparrow$ \\
\hline Ethanol & $64-17-5$ & $\mathrm{C} 2 \mathrm{H} 6 \mathrm{O}$ & 46.04 & 2.26 & $\uparrow$ \\
\hline Acetone & $67-64-1$ & $\mathrm{C} 3 \mathrm{H} 6 \mathrm{O}$ & 58.04 & 2.59 & $\downarrow$ \\
\hline Isopropyl Alcohol & $67-64-1$ & $\mathrm{C} 3 \mathrm{H} 8 \mathrm{O}$ & 60.06 & 2.61 & $\downarrow$ \\
\hline Acrolein & $107-02-8$ & $\mathrm{C} 3 \mathrm{H} 4 \mathrm{O}$ & 56.03 & 2.55 & $\downarrow$ \\
\hline Furan, tetrahydro- & 109-99-9 & $\mathrm{C} 4 \mathrm{H} 8 \mathrm{O}$ & 72.06 & 5.41 & $\downarrow$ \\
\hline Heptane & $142-82-5$ & C7H16 & 100.13 & 7.25 & $\uparrow$ \\
\hline Ethylbenzene & $100-41-4$ & C8H10 & 106.08 & 11.49 & $\uparrow$ \\
\hline Carane & $17530-24-4$ & C10H18 & 138.14 & 14.30 & $\uparrow$ \\
\hline Dodecane & $112-40-3$ & $\mathrm{C} 12 \mathrm{H} 26$ & 170.20 & 17.47 & $\downarrow$ \\
\hline Tetradecane & $629-59-4$ & C14H30 & 198.23 & 20.46 & $\uparrow$ \\
\hline Tetradecanal & $124-25-4$ & $\mathrm{C} 14 \mathrm{H} 28 \mathrm{O}$ & 212.37 & 23.18 & $\uparrow$ \\
\hline
\end{tabular}

ethanol. No human- or bacteria-specific Wikipathways were found.

\subsection{Discussion}

In the present study, VOC profiles were determined in exhaled breath of patients clinically suspected of VAP to discriminate patients with VAP from other critically ill ventilated patients. Of 100 patients, 32 were diagnosed with VAP by quantitative BAL analysis. This ratio was in line with earlier publications [20,33]. A discriminating profile of 12 exhaled VOCs was identified that could determine the presence of VAP with an accuracy of $74.2 \% \pm 13.8 \%$, accompanied by a sensitivity of $75.8 \% \pm 13.5 \%$, a specificity of $73.0 \% \pm 11.8 \%$ and an AUC of 0.87 . The 12 VOCs that were identified by the model were chemically diverse. There was no significant difference in the diagnosis at admission or in the frequency and distribution of comorbidities between the $\operatorname{VAP}(+)$ and $\operatorname{VAP}(-)$ group of patients. However, a haematological diagnosis at admission and active malignancy as comorbidity were more prevalent among $\operatorname{VAP}(-)$ patients. Several potential confounders, including haematological diagnosis and active malignancies, were tested and proven not to be significantly associated with the VOC profile. The predominance of male patients in the demographics of the present study might reflect known gender differences in the incidence of sepsis and VAP $[34,35]$.

These results demonstrate the potential of exhaled breath as a diagnostic tool in the ICU, where less invasive and faster detection methods are of great importance. Although the results are encouraging, the external validation in a large, multicentre cohort is necessary for clinical application. The advantage of exhaled breath over BAL 


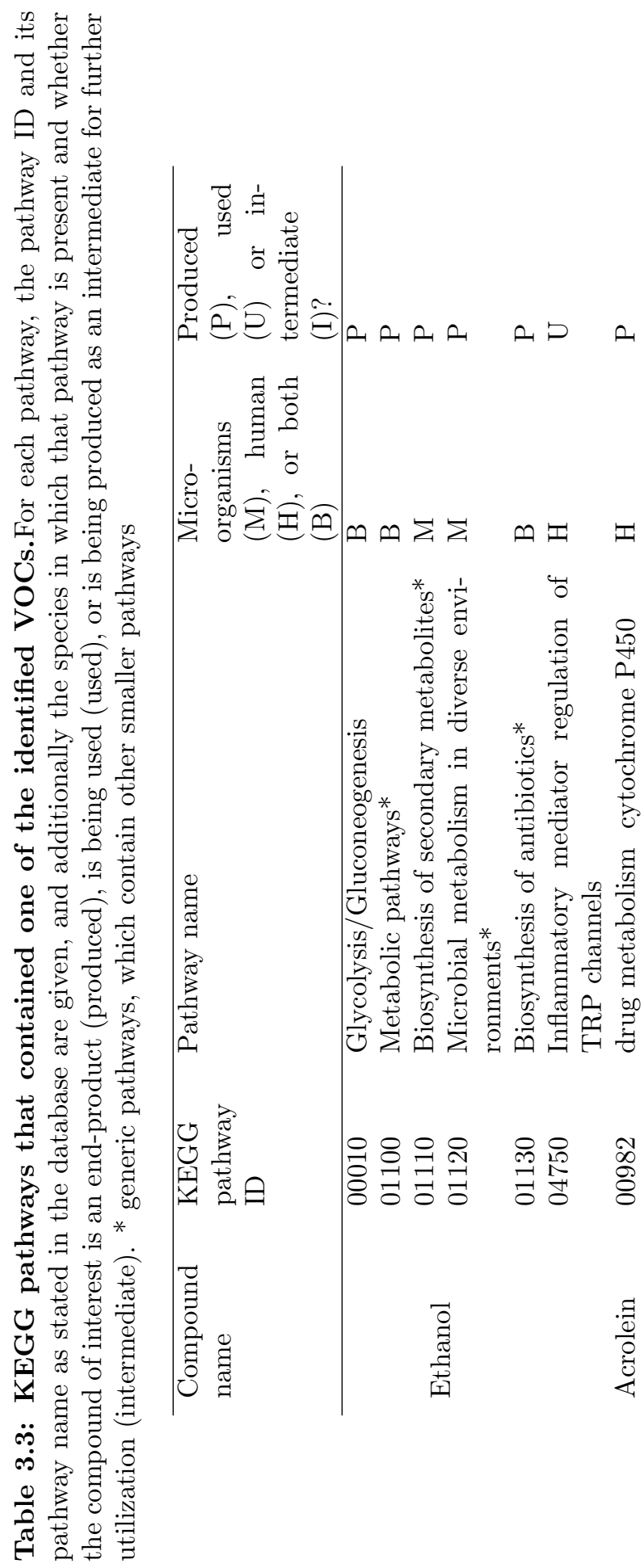


analysis - the current gold standard for diagnosis of VAP - is that it is easy to perform, non-invasive and can be analysed within a short time span. In contrast to BAL, where the time to diagnosis is at least $48 \mathrm{~h}$, exhaled breath sampling could take as little as one hour to get a diagnosis. To achieve this, exhaled breath, upon sampling after clinical suspicion of VAP, should be immediately transferred to a laboratory where it has to be processed by a mass spectrometer right away and subsequently checked for markers of VAP using a predesigned and validated algorithm. Eventually, such a fast diagnostic tool could support tailored antibiotic treatment, thereby aiding antibiotic resistance [4]. Additionally, it could reduce hospital costs and medication use [36].

Thus far, most of the research done on exhaled breath in diagnosis of VAP was done with the e-nose technology. One study tested the use of e-nose technology as a substitute for chest computed tomography scan as a diagnostic tool for diagnosing VAP [37]. Although a prediction value of $80 \%$ was discovered, these results are difficult to interpret correctly due to the lack of independent validation.

A more recent study by the same authors evaluated the use of the e-nose as a substitute for the Clinical Pulmonary Infection Score (CPIS) [38]. The CPIS is a measure of pulmonary infection that is used to diagnose VAP with an arbitrary cutoff of 6 for the diagnosis $[>6=\operatorname{VAP}(+),<6=\operatorname{VAP}(-)]$. However, since the CPIS is considered to be not reliable enough to diagnose VAP in the clinical setting [39], it is unclear whether the included patients were accurately diagnosed with VAP, which consequently skews their findings.

Bos et al. also performed a prospective cohort study on diagnosing VAP with the e-nose [40]. They collected tracheal aspirates (TAs), and successively analysed the headspace of a bacterial culture medium of these TAs. They found an AUC of 0.85 in their cross-sectional study, which is comparable to our observed AUC of 0.87 . All of these studies utilize the e-nose technology which has some limitations including reproducibility, negative effects of temperature and humidity, and the inability to identify the chemical identity of VOCs underlying the disease [41]. Knowing the identity of a VOC enables us to look at the underlying biological mechanisms of the disease.

Acute respiratory distress syndrome (ARDS) describes a condition of severe lung failure that can be caused by various non-infectious and infectious diseases including VAP. Moreover, mechanical ventilation in patients with ARDS can also facilitate the development of VAP. Exhaled breath as a diagnostic marker of ARDS was recently tested and could correctly classify ARDS patients and controls with an AUC of 0.78 [42]. In our study, an AUC of 0.87 was found for VAP, which suggests that VAP may produce more pronounced differences in the exhaled breath. The ARDS study was performed using GC-MS and identified three VOCs to be essential for the discriminating model, of which two alkanes and one aldehyde. Although none of the individual compounds corresponded to the VOCs identified in our study, we found alkanes and aldehydes as well. This could imply involvement of similar underlying biological mechanisms in VAP and ARDS as well as sufficient differences between the pathology of the conditions to make them distinguishable by exhaled breath.

Over the last few years, multiple studies were performed to identify VOCs specific to a certain strain of bacteria. A recent review summarized all VOCs found for the six most frequently found bacteria in the ICU: Staphylococcus aureus, Streptococcus 
pneumoniae, Enterococcus faecalis, Pseudomonas aeruginosa, Klebsiella pneuomoniae and Escherichia coli [7]. These are all known to cause VAP in critically ill patients [43]. Another recent study detected VOCs that could differentiate various species of bacteria in vitro [14]. Although some of the bacteria included in this study were similar to those present in the current VAP patients, no overlap between the discriminating VOCs was observed. Aside from the small number of patients affected by each of these individual bacteria, this lack in overlap could also be explained by the fact that the in vitro study compared strains among one another whereas the present study compared diseased patients and controls. More generally, all of the studies described here were performed in vitro or ex vivo whereas our study has been carried out with in vivo samples from a very heterogeneous patient population. This may also explain the discrepancies with our findings, as there are limitations to the translatability of in vitro and ex vivo experiments into an in vivo situation [44].

Recently a study was published by Fowler et al. where 46 ICU patients with sterile brain injury were followed as some of them developed a significant presence of airway pathogens $(>104 \mathrm{cfu} / \mathrm{mL})$ that ultimately may have led to VAP in these patients [16]. Their exhaled breath was sampled and analysed in a similar manner as in our study using thermal desorption coupled with gas chromatography time of flight mass spectrometry, followed by multivariate analysis of the data. Likewise, BAL results were used as a reference in the diagnosis of VAP. Remarkably, $33 \%$ of the monitored patients demonstrated significant growth of pathogens in the lower respiratory tract. Hence, the incidence of VAP was much higher than in our ICU where we found an average of 2.5 episodes of VAP in 1,000 ventilator days [45]. This discrepancy might be explained by a higher risk of aspirations in patients with brain injury requiring intubation [46]. Although the patient population may not adequately reflect the overall ICU population, the results from Fowler et al. are very promising as a means to associate bacterial colonisation of the lower respiratory tract with exhaled VOCs. In contrast, our study reflects more the current clinical guidelines in the diagnosis of VAP, and the heterogeneity of an ICU population.

The list of VOCs found for $\operatorname{VAP}(+)$ vs. $\operatorname{VAP}(-)$ comparison consists of both endogenous and exogenous sources. Some endogenous compounds may be useful as they can indicate the cellular processes underlying VAP.

Ethanol is a compound that is both produced by processes specific for bacteria, but also during glycolysis and gluconeogenesis pathway, that are present in both the human host and the bacteria. In all but one of these pathways it is produced as a metabolite end-product, which is reflected in the increased level of exhaled ethanol in $\operatorname{VAP}(+)$ patients.

Acetone is produced by spontaneous decarboxylation of acetoacetate, which is produced as a result of the build-up of ketone bodies. These ketones can be formed in the liver as a result of sepsis [47]. As a consequence of acetone build-up, isopropyl alcohol is formed as break-down product of acetone during ketogenesis. The exhaled concentrations of both compounds were lower in $\operatorname{VAP}(+)$ compared to $\operatorname{VAP}(-)$ patients. This can be explained by the fact that ketogenesis is reduced during inflammatory or infectious states [47], resulting in less production of acetone and isopropyl alcohol.

Acrolein (2-propenal) is also very likely to originate from endogenous sources and can be produced by a number of cellular processes. Firstly, lipid peroxidation accounts 
for a small portion of the endogenously produced acrolein. Secondly, myeloperoxidase (MPO),0 playing a crucial role in oxidative stress and the immune response to bacteria, was found to oxidize threonine into acrolein. Lastly, polyamines can also be catabolized into acrolein [48]. These polyamines are essential to the cell as they influence a range of processes from RNA and DNA structure to enzyme activity [49]. Additionally, one KEGG pathway was found where acrolein is formed as a breakdown product of anti-cancer drugs. Each group did include a few patients that were administered cyclophosphamide, however the percentage of these patients did not differ between $\operatorname{VAP}(+)$ and $\operatorname{VAP}(-)$ group. It therefore seems likely that the different abundance in acrolein originates from one of the described endogenous pathways.

Five of the 12 VOCs identified by the model can be classified as (branched) alkanes: heptane, 2-methylbutane, dodecane, tetradecane and tetradecanal. Two primary processes could account for the presence of alkanes in exhaled breath. First, lipid peroxidation as a result of oxidative stress is able to produce hydrocarbons. Heptane is thought to originate from oleic acid, and 2-methylbutane may originate from 2-methyl-1,3-butadiene (also known as isoprene). Second, alkanes are also present in the environment and are inhaled on a daily basis. After ingestion, the compounds are broken down in the liver by cytochrome P450 enzymes (CYP). The activity of these enzymes decreases with aging, but also with disease, implicating reduced CYP activity in severely ill patients [50]. Both mechanisms could explain the different abundances found between $\operatorname{VAP}(+)$ and $\operatorname{VAP}(-)$. Ethylbenzene is an benzene derivative and an indoor pollutant [51]. Benzene and its derivatives are also broken down in the liver by CYP enzymes, which may not function properly in critically ill patients, resulting in different exhaled abundances of ethylbenzene in $\operatorname{VAP}(+)$ vs. $\operatorname{VAP}(-)$ patients [52]. The two remaining compounds, tetrahydrofuran and carane $(3,7,7$-Trimethylbicyclo [4.1.0]heptane), are also environmental pollutants and have no known endogenous source. Both compounds are likely metabolized by CYP enzymes in the liver, but no literature was found that supports this theory.

A limitation of the present study is the relatively small number of subjects. We were unable to test for specific strains of bacteria. As VAP is generally caused by an array of bacteria, we only had a few patients per bacterial strain available at most, hindering the use of multivariate statistics to identify strain-specific VOCs in vivo.

Although the quantitative culture analysis of BAL is accepted as state-of-the art in the diagnosis of VAP [53], the sensitivity and specificity were variable among earlier histopathology studies with percentages of $42-93 \%$ and $45-100 \%$, respectively [5456]. This could have led to misdiagnosing several patients which could have possibly influenced the findings of the current study. However, the use of RF as multivariate technique reduces the influence of mislabelled samples on the outcome.

\subsection{Conclusion}

The present study has demonstrated that it is possible to distinguish ICU patients with VAP from patients without VAP based on a profile of only 12 VOCs. Exhaled breath analysis is a promising, simple, safe and non-invasive technique for the rapid diagnosis of VAP. A larger study population is warranted to confirm our findings. 
Additionally, studies should be performed where strain-specific VOC profiles can be found.

\subsection{Supplementary Materials}

Table 3.S1: Statistical significance of confounding factors tested by rMANOVA. The presence of one or more comorbidities was tested as well as the presence of specific comorbidities. None of the potential confounding factors were significantly associated with the VOC profile.

\begin{tabular}{ll}
\hline Confounder & $\mathrm{p}$-value \\
\hline Age & 0.5400 \\
Gender & 0.3750 \\
SOFA score & 0.1970 \\
ICU mortality & 0.2160 \\
In hospital mortality & 0.3030 \\
Diagnostic group upon admission & 0.2370 \\
Severe sepsis & 0.2070 \\
Comorbidities (present yes/no) & 0.1790 \\
Respiratory & 0.4090 \\
Chronic renal failure & 0.9460 \\
Cardiovascular & 0.1590 \\
Active malignancy & 0.4200 \\
Immunocompromised & 0.7040 \\
Chronic liver failure & 0.2320 \\
Neurological impairment & 0.3020
\end{tabular}




\section{Bibliography}

[1] Amédée Ego, Jean Charles Preiser, and Jean Louis Vincent. Impact of diagnostic criteria on the incidence of ventilator-associated pneumonia. Chest, 147(2):347-355, 2015.

[2] Wilhelmina G. Melsen, Maroeska M. Rovers, Rolf H H Groenwold, et al. Attributable mortality of ventilator-associated pneumonia: A meta-analysis of individual patient data from randomised prevention studies. The Lancet Infectious Diseases, 13(8):665-671, 2013.

[3] J Y Fagon, Jean Chastre, Michel Wolff, et al. Invasive and noninvasive strategies for management of suspected ventilator-associated pneumonia. A randomized trial. Annals of internal medicine, 132(8):621-30, 2000.

[4] K Bush, P Courvalin, G Dantas, et al. Tackling antibiotic resistance. Nat Rev Microbiol, 9(12):894-896, dec 2011.

[5] Wolfram Miekisch, Jochen K Schubert, and Gabriele F.E Noeldge-Schomburg. Diagnostic potential of breath analysisfocus on volatile organic compounds. Clinica Chimica Acta, 347(1):25-39, sep 2004.

[6] Agnes W Boots, Joep J B N van Berkel, Jan W Dallinga, et al. The versatile use of exhaled volatile organic compounds in human health and disease. Journal of Breath Research, 6(2):27108, jun 2012.

[7] Lieuwe D. J. Bos, Peter J. Sterk, Marcus J. Schultz, L Hofmann, and S Ulbricht. Volatile Metabolites of Pathogens: A Systematic Review. PLoS Pathogens, 9(5):e1003311, may 2013.

[8] J. J B N Van Berkel, J. W. Dallinga, G. M. Möller, et al. A profile of volatile organic compounds in breath discriminates COPD patients from controls. Respiratory Medicine, 104(4):557-563, apr 2010.

[9] J. W. Dallinga, C. M H H T Robroeks, J. J B N Van Berkel, et al. Volatile organic compounds in exhaled breath as a diagnostic tool for asthma in children. Clinical and Experimental Allergy, 40(1):68-76, jan 2010.

[10] Charlotte M. Robroeks, Joep J. Van Berkel, Quirijn Jöbsis, et al. Exhaled volatile organic compounds predict exacerbations of childhood asthma in a 1-year prospective study. European Respiratory Journal, 42(1):98-106, 2013.

[11] A. H J Kolk, J. J B N Van Berkel, M. M. Claassens, et al. Breath analysis as a potential diagnostic tool for tuberculosis. International Journal of Tuberculosis and Lung Disease, 16(6):777-782, 2012.

[12] Charlotte M H H T Robroeks, Joep J B N van Berkel, Jan W. Dallinga, et al. Metabolomics of volatile organic compounds in cystic fibrosis patients and controls. Pediatric research, 68(1):7580, jul 2010. 
[13] Agnieszka Smolinska, Ester M. M. EM Klaassen, JW Jan W. Dallinga, et al. Profiling of volatile organic compounds in exhaled breath as a strategy to find early predictive signatures of asthma in children. PLoS ONE, 9(4):e95668, apr 2014.

[14] a W Boots, A Smolinska, J.J.B.N. van Berkel, et al. Identification of microorganisms based on gas chromatography-mass spectrometric analysis of volatile organic compounds in headspace gases. Journal of biomedicine E biotechnology, 8(2):1752, jun 2014.

[15] Wojciech Filipiak, Andreas Sponring, Maria M. Baur, et al. Characterization of volatile metabolites taken up by or released from Streptococcus pneumoniae and Haemophilus influenzae by using GC-MS. Microbiology (United Kingdom), 158(12):3044-3053, dec 2012.

[16] S. J. Fowler, M. Basanta-Sanchez, Y. Xu, R. Goodacre, and P. M. Dark. Surveillance for lower airway pathogens in mechanically ventilated patients by metabolomic analysis of exhaled breath: a case-control study. Thorax, 70(4):320-325, apr 2015.

[17] CCMO Help-me-on-my-way.

[18] E. I G B De Brauwer, Jan A. Jacobs, Fred Nieman, Cathrien A. Bruggeman, and Marjolein Drent. Bronchoalveolar lavage fluid differential cell count: How many cells should be counted? Analytical and Quantitative Cytology and Histology, 24(6):337-341, 2002.

[19] Catharina F M Linssen, Otto Bekers, Marjolein Drent, and Jan a Jacobs. C-reactive protein and procalcitonin concentrations in bronchoalveolar lavage fluid as a predictor of ventilatorassociated pneumonia. Annals of clinical biochemistry, 45(Pt 3):293-298, 2008.

[20] Catharina F M Linssen, Jan A. Jacobs, Jan S A G Schouten, et al. Influence of antibiotic therapy on the cytological diagnosis of ventilator-associated pneumonia. Intensive Care Medicine, 34(5):865-872, 2008.

[21] A Smolinska, A-Ch C Hauschild, R R R Fijten, et al. Current breathomicsa review on data pre-processing techniques and machine learning in metabolomics breath analysis. Journal of breath research, 8(2):027105, jun 2014.

[22] Leo Breiman. Random forests. Mach. Learn., 45(1):5-32, oct 2001.

[23] J A Hanley and B J McNeil. The meaning and use of the area under a receiver operating characteristic (ROC) curve. Radiology, Illinois, 143(1):29-36, apr 1982.

[24] J. Engel, L. Blanchet, B. Bloemen, et al. Regularized MANOVA (rMANOVA) in untargeted metabolomics. Analytica Chimica Acta, 899:1-12, oct 2015.

[25] Janna Hastings, Paula De Matos, Adriano Dekker, et al. The ChEBI reference database and ontology for biologically relevant chemistry: Enhancements for 2013. Nucleic Acids Research, 41(D1):D456-63, 2013.

[26] Kristina M Hettne, Antony J Williams, Erik M van Mulligen, et al. ChemSpider: An Online Chemical Information Resource. Journal of cheminformatics, 2(1):3, 2010.

[27] Evan E. Bolton, Yanli Wang, Paul a. Thiessen, and Stephen H. Bryant. PubChem: Integrated Platform of Small Molecules and Biological Activities. Annual Reports in Computational Chemistry, 4:217-241, 2008.

[28] Martijn P van Iersel, Alexander R Pico, Thomas Kelder, et al. The BridgeDb framework: standardized access to gene, protein and metabolite identifier mapping services. BMC bioinformatics, 11:5, 2010.

[29] E L Willighagen. Accessing biological data in $\mathrm{R}$ with semantic web technologies. PeerJ PrePrints, 2104.

[30] Thomas Kelder, Martijn P. Van Iersel, Kristina Hanspers, et al. WikiPathways: Building research communities on biological pathways. Nucleic Acids Research, 40(D1):D1301-D1307, jan 2012.

[31] Minoru Kanehisa, Susumu Goto, Yoko Sato, et al. Data, information, knowledge and principle: Back to metabolism in KEGG. Nucleic Acids Research, 42(D1):D199-205, jan 2014.

[32] Johannes B J Scholte, Helke A. Van Dessel, Catharina F M Linssen, et al. Endotracheal aspirate and bronchoalveolar lavage fluid analysis: Interchangeable diagnostic modalities in suspected ventilator-associated pneumonia? Journal of Clinical Microbiology, 52(10):3597-3604, 2014. 
[33] G. U. Meduri, G. L. Mauldin, R. G. Wunderink, et al. Causes of fever and pulmonary densities in patients with clinical manifestations of ventilator-associated pneumonia. Chest, 106(1):221-235, 1994.

[34] Yasser Sakr, Cristina Elia, Luciana Mascia, et al. The influence of gender on the epidemiology of and outcome from severe sepsis. Critical care (London, England), 17(2):R50, 2013.

[35] John P Sharpe, Louis J Magnotti, Jordan a Weinberg, et al. Gender disparity in ventilatorassociated pneumonia following trauma: identifying risk factors for mortality. The journal of trauma and acute care surgery, 77(1):161-5, 2014.

[36] Nasia Safdar, Cameron Dezfulian, Harold R Collard, and Sanjay Saint. Clinical and economic consequences of ventilator-associated pneumonia: a systematic review. Critical care medicine, 33(10):2184-2193, oct 2005.

[37] Neil G Hockstein, Erica R Thaler, Drew Torigian, et al. Diagnosis of pneumonia with an electronic nose: correlation of vapor signature with chest computed tomography scan findings. The Laryngoscope, 114(10):1701-5, 2004.

[38] C William Hanson and Erica R Thaler. Electronic nose prediction of a clinical pneumonia score: biosensors and microbes. Anesthesiology, 102(1):63-68, jan 2005.

[39] MaryaD. Zilberberg and AndrewF. Shorr. VentilatorAssociated Pneumonia: The Clinical Pulmonary Infection Score as a Surrogate for Diagnostics and Outcome. Clinical Infectious Diseases, 51(S1):S131-S135, aug 2010.

[40] Lieuwe D. J. Bos, Ignacio Martin-Loeches, Janine B. Kastelijn, et al. The volatile metabolic fingerprint of ventilator-associated pneumonia. Intensive Care Medicine, 40(5):761-762, may 2014.

[41] a.D Wilson and M. Baietto. Applications and advances in electronic-nose technologies. Sensors, $9(7): 5099-5148$, jan 2009.

[42] Lieuwe D J Bos, Hans Weda, Yuanyue Wang, et al. Exhaled breath metabolomics as a noninvasive diagnostic tool for acute respiratory distress syndrome. European Respiratory Journal, 44(1):188-197, jul 2014.

[43] David R Park. The Microbiology of Ventilator-Associated Pneumonia Features of Specific Common VAP Pathogens The Prevalence of Routine Bacterial Pathogens in VAP VAP in Patients After Tracheotomy VAP in Patients With Traumatic Injuries. Respiratory care, 50(6):742-765, jun 2005.

[44] Jiangjiang Zhu, Heather D Bean, Matthew J Wargo, Laurie W Leclair, and Jane E Hill. Detecting bacterial lung infections: in vivo evaluation of in vitro volatile fingerprints. Journal of breath research, 7(1):16003, mar 2013.

[45] Ronny M Schnabel, Johannes B J Scholte, Kim E H M Van Der Velden, Paul M H J Roekaerts, and Dennis C J J Bergmans. Ventilator-associated pneumonia rates after introducing selective digestive tract decontamination. Infectious diseases (London, England), 4235(November):1-4, 2015.

[46] Kiwon Lee and Fred Rincon. Pulmonary complications in patients with severe brain injury. Critical Care Research and Practice, 2012:207247, 2012.

[47] T C Vary, J H Siegel, T Nakatani, T Sato, and H Aoyama. A biochemical basis for depressed ketogenesis in sepsis. J Trauma, 26(5):419-425, 1986.

[48] Jan F. Stevens and Claudia S. Maier. Acrolein: Sources, metabolism, and biomolecular interactions relevant to human health and disease. Molecular Nutrition and Food Research, 52(1):7-25, 2008.

[49] Anthony E. Pegg. Mammalian polyamine metabolism and function. IUBMB Life, 61(9):880$894,2009$.

[50] J Kwak and G Preti. Volatile disease biomarkers in breath: a critique. Curr Pharm Biotechnol, 12(7):1067-1074, 2011.

[51] Kaoru Midorikawa, Takafumi Uchida, Yoshinori Okamoto, et al. Metabolic activation of carcinogenic ethylbenzene leads to oxidative DNA damage. Chemico-Biological Interactions, 150(3):271-281, dec 2004. 
[52] Craig Sams, George D. Loizou, John Cocker, and Martin S. Lennard. Metabolism of ethylbenzene by human liver microsomes and recombinant human cytochrome P450s (CYP). Toxicology Letters, 147(3):253-260, mar 2004.

[53] Jean-Yves Fagon. Diagnosis and treatment of ventilator-associated pneumonia: fiberoptic bronchoscopy with bronchoalveolar lavage is essential. Seminars in respiratory and critical care medicine, 27(1):34-44, 2006.

[54] A. Torres and M. El-Ebiary. Bronchoscopic BAL in the diagnosis of ventilator-associated pneumonia. Chest, 117(4 SUPPL. 2):198S-202S, 2000.

[55] J. Chastre, J. Y. Fagon, M. Bornet-Lecso, et al. Evaluation of bronchoscopic techniques for the diagnosis of nosocomial pneumonia. American Journal of Respiratory and Critical Care Medicine, 152(1):231-240, 1995.

[56] C. H. Marquette, M. C. Copin, F. Wallet, et al. Diagnostic tests for pneumonia in ventilated patients: Prospective evaluation of diagnostic accuracy using histology as a diagnostic gold standard. American Journal of Respiratory and Critical Care Medicine, 151(6):1878-1888, 1995. 
CHAPTER 4

The use of exhaled air analysis in discriminating interstitial lung diseases: a pilot study

R. Fijten, L. Plantier, C. Clerici, A. Smolinska, M. Flamant, J. Dallinga, J. Marcadier, D. Pachen, M. dOrtho, F. van Schooten, B. Crestani, A. Boots Manuscript in preparation 


\subsection{Abstract}

Interstitial lung diseases (ILDs) are a heterogeneous group of chronic lung diseases characterized by lung inflammation and tissue remodeling. They include idiopathic ILDs such as idiopathic pulmonary fibrosis (IPF) and ILDs secondary to chronic granulomatous diseases such as sarcoidosis and connective tissue disease (CTD). As differential diagnosis of these diseases remains difficult and invasive, the primary aim is to identify whether volatile organic compounds (VOCs) in exhaled air can be used to non-invasively discriminate IPF and CTD-ILD. As secondary aim, the association between the unique IPF and CTD-ILD VOC patterns and lung function decline is investigated.

Fifty-one IPF patients, 53 CTD-ILD patients and 88 healthy controls were asked to donate exhaled air which was analyzed for its VOC content using gas chromatographtime of flight- mass spectrometry. By applying multivariate analysis, a discriminative profile of 34 VOCs was observed to discriminate between IPF patients and healthy controls whereas 11 unique VOCs were selected to distinguish between CTD-ILD patients and healthy controls. The separation between the two ILDs could be made using 16 discriminating volatiles, that also displayed a significant correlation with total lung capacity and the 6 minutes walk distance.

This study reports for the first time that unique VOC profiles can be detected to differentiate IPF and CTD-ILD from both healthy controls and each other. Moreover, an ILD-specific VOC profile was strongly correlated with clinical parameters. Future research applying larger cohorts of patients suffering from a larger variety of ILDs should confirm the potential use of breathomics to facilitate fast, non-invasive and proper differential diagnosis of specific ILDs in the future as first step towards personalized medicine for these complex diseases.

\subsection{Introduction}

Interstitial lung diseases (ILDs) are a heterogeneous group of chronic lung diseases characterized by varying degrees of lung inflammation, resulting from the activation of both the innate and adaptive immune system, and tissue remodeling culminating in lung fibrosis. Most frequent ILDs include idiopathic ILDs such as idiopathic pulmonary fibrosis (IPF) and ILDs secondary to chronic granulomatous diseases, such as sarcoidosis and connective tissue disease (CTD), exemplified by systemic sclerosis (SSc). Although CTD-ILD is often considered as a single entity, this term actually reflects a heterogeneous spectrum of diverse CTDs and a variety of patterns of interstitial pneumonia [1].

Due to their relative low incidence and prevalence numbers, ILDs are still an under recognized problem within universal health care systems. However, these diseases induce overwhelming morbidity and are the leading cause of mortality in patients suffering from CTD $[2,3]$. Moreover, if classified as a malignancy, IPF would rank as the eighth most prevalent cancer worldwide [4].

Recent advances in the understanding of ILD pathogenesis suggest that general 
pathways underlie the processes leading to the typical destruction of the lung architecture observed in these diseases [4]. Characteristically, these pathways are set into motion by intrinsic defects in the wound healing response of lung epithelial cells and fibroblasts [5], followed by a defective re-epithelisation and fibroblast hyperproliferation [5]. Additionally, the formation of hyperplastic pneumocytes at the fibroblastic foci and excessive accumulation of extracellular matrix components will also contribute to the ultimate scar formation typical for IPF [6]. A well-recognized key mediator in the pathogenesis of IPF, as well as other ILDs is oxidative stress, a state that can be defined as an imbalance between the production of and the protection against reactive oxygen species (ROS) in favor of the first [7, 8]. Interestingly, an active inflammatory response surely can [9] but does not necessarily [10] have to contribute to ILD pathology as well, thereby explaining why anti-inflammatory drugs are not effective in all different ILDs. Although these pathways are thought to be rather common for mediating end-stage lung fibrosis and destructing lung tissue in ILDs, initial pathogenic mechanisms may be specific to each type of ILD.

Likewise, both prognosis and response to therapeutics vary widely among ILDs. IPF is characterized by a poor prognosis and survival rates that, from time of diagnosis, are comparable to those of inoperable pulmonary malignancies [4]. Despite the contribution of inflammation to IPF pathogenesis, there is a lack of beneficial effect of immunosuppressive therapeutics [11]. Recent trials have shown that the two anti-fibrotic drugs nintedanib and pirfenidone are able to slow down IPF progression and functional decline, but there is still no evidence that any currently available treatment option is able to stop the progression or ideally even revert the process of this disease [4, 12-14]. Other ILD including sarcoidosis, Ssc-associated pneumonia and CTD-ILD carry a somewhat better prognosis and are usually responsive to glucocorticoids or immunosuppressive agents including mycophenolate mofetil and azathioprine, although a significant minority of CTD-ILD patients does not tolerate the latter due to severe side effects [15-17]. Within these other interstitial lung disease, the development of new drugs is challenged especially for the CTD-ILD subgroup due to the unpredictable course and presentation of this class of diseases combined with its considerable mortality [18]. Therefore it is of crucial importance to obtain a differential diagnosis at the earliest possible moment to steer the appropriate medication per subgroup.

As a result of these remarkable differences in progression and especially treatment of the various ILDs, it is highly important to diagnostically distinct IPF and other forms of ILD. The difficulty of distinguishing CTD-ILD from other ILDs including IPF can be exemplified by the facts that up to $15 \%$ of ILD patients also present CTD symptoms during their initial evaluation whereas up to $25 \%$ of ILDs occur in patients with undiagnosed CTD [19]. Despite widespread adoption of advanced tools such as high-resolution computed tomography (HRCT) scans and availability of disease-specific hematological markers, differential diagnosis in ILD patients remains difficult and often mandates invasive procedures such as surgical lung biopsies [20]. Indeed, although such a surgical biopsy is no longer considered the diagnostic golden standard in ILD, it is still the single most informative test in cases where both the clinical and HCRT features fail to provide an exclusive diagnosis [21]. However, due to a number of contraindications including age, comorbidities and the severity of the 
disease, it is not always possible to obtain such a diagnostic biopsy, underlining the importance to develop other diagnostic markers that are less or even non-invasive, safe and fast such as cryobiopsies and markers of oxidative stress [21-23]. Besides new diagnostic markers, there is also an urgent need for biomarkers of disease progression and therapeutic efficacy in order to phenotype ILD patients. Currently, monitoring of ILD patients relies mostly on clinical, morphological and functional criteria, which may lack sensitivity in detecting early and/or minimal changes in disease activity. It can be anticipated that developing biomarkers of disease progression and therapy efficacy will aid in creating subgroups of patients based on their disease severity and response to therapy as a first step towards precision medicine in ILD [24].

An alternative approach for the non-invasive differential diagnosis of ILD could be the analysis of exhaled air, known to contain a complex mixture of volatile organic compounds (VOCs) that might be applied as potential biomarkers for chronic lung diseases $[25,26]$. To this extent, we have developed a sampling methodology for collecting concentrated samples of exhaled air against which a two-stage thermal desorption gas chromatography-time-of flight mass spectrometry (GC-MS) analysis has been employed [25]. Additionally, data analysis tools have been developed to enable the pipeline analysis of the generated GC-MS sample outputs [27]. By extracting informative VOCs from the compiled database and implementing them into a classifier of which the performance was evaluated [29], we have already shown that a specific VOC profile in breath can discriminate healthy controls from patients suffering from a variety of lung diseases including chronic obstructive pulmonary disease, ventilatedassociated pneumonia and asthma [28-30]. However, it would clinically be more relevant to apply exhaled VOCs in differentiating between chronic lung diseases that (partly) share pathogenesis and symptoms yet display diverse outcomes and therefore require different treatment options. Therefore, the primary aim of the present study is to identify exhaled VOC profiles characteristic for idiopathic and secondary ILDs, represented by IPF and CTD-ILD respectively, in comparison to patients without lung disease as well as to each other. Such a unique volatile profile for the different ILDs could represent a novel diagnostic tool for positive and differential diagnosis of these complex diseases. Moreover, the secondary aim of this study is to investigate whether these exhaled VOCs, specific for either IPF or CTD-ILD, could be associated with lung function decline and thus serve as breath biomarkers of prognosis.

\subsection{Materials \& Methods}

A monocentric case-control observational study was performed using patients referred for lung, renal or heart function testing to the Physiology Department of the BichatClaude Bernard university hospital (Paris, France). Between 2014 and 2016, patients were recruited at the time of their visit to this hospital by their own physician. All participants were fully informed, both written and orally, about the aim and details of the study and have given their written informed consent. Prior to the inclusion, the protocol of this study was approved by the Observational Research Protocol Evaluation Committee (CEPRO) of the French Respiratory Society (SPLF). 


\subsubsection{Patients}

The study included a total of 224 subjects, divided into a group of 88 controls and 136 interstitial lung diseases (ILD) patients. Due to unequal and too low inclusion within some specific ILDs, the study ultimately focused on two specific groups of ILD patients, i.e. subjects suffering from idiopathic pulmonary fibrosis (IPF; $n=53$ ) or from connective tissue disease-associated ILD (CTD-ILD; $\mathrm{n}=51$ ). Both groups of patients were recruited among subjects with known and well-characterized ILD, all referred to the university hospital for lung function testing. Specific ILD diagnosis was ascertained by reviewing medical charts established by the Referral Center for Rare Lung Diseases (Service de Pneumologie A, Paris, France). Lung function and HRCT data were obtained for patients in the two ILD groups. Control subjects were recruited among patients referred for assessment of hypertension, polysomnography or coronary heart disease (cardiac controls, $\mathrm{n}=37$ ) as well as among patients referred for renal testing (renal controls; $\mathrm{n}=51$ ).

\subsubsection{Inclusion criteria}

For the control group, inclusion criteria combined an age between 40 and 80 years and a lack of 1) any known respiratory disease including asthma earlier in life, 2) significant exertional dyspnea (Medical Research Council scale 0-1), 3) inhaled medications, 4) any known chronic liver disease, HIV infection, diabetes, inflammatory bowel diseases and 5) congestive heart failure. For both the IPF and CTD-ILD group, the age was also set between 40 and 80 years and combined with a lack of any known chronic liver disease, HIV infection, diabetes, inflammatory bowel diseases and congestive heart failure. Additionally, IPF was diagnosed according to ERS/ATS/JAS/ALAT criteria (Raghu et al, 2011) whereas CTD-ILD was identified by confirming ILD at HRCT and CTD (rheumatoid arthritis, Sjgrens syndrome, polymyositis/dermatomyositis) according to ACR criteria.

\subsubsection{Judgement criteria}

All subjects were assigned to either the control or ILD group based on their clinical data with an emphasis on MRC dyspnea scale and the presence of chronic cough. Subsequently, ILD patients were characterized by lung function data and HRCT data. For the first, spirometry (vital capacity (VC) and forced expiratory volume in 1 second (FEV1)), static volumes (total lung capacity (TLC) and carbon monoxide diffusion capacity (TLCO using the single breath method) were analyzed. For the HRCT data, the presence of a usual interstitial pneumonia (UIP)pattern, possible UIP pattern or the incompatibility with an UIP pattern was determined according to the 2011 ATS/ERS/JRS/ALAT statement [33].

The characteristics of all subjects, both controls and ILD patients, are summarized in Table 4.1. For each of the study parameters, a students t-test was performed to check for significant differences between two groups. Multiple testing correction was performed using the False Discovery Rate correction [31].s The degree of significance can be found in Table 4.S1 of the supplementary material. 


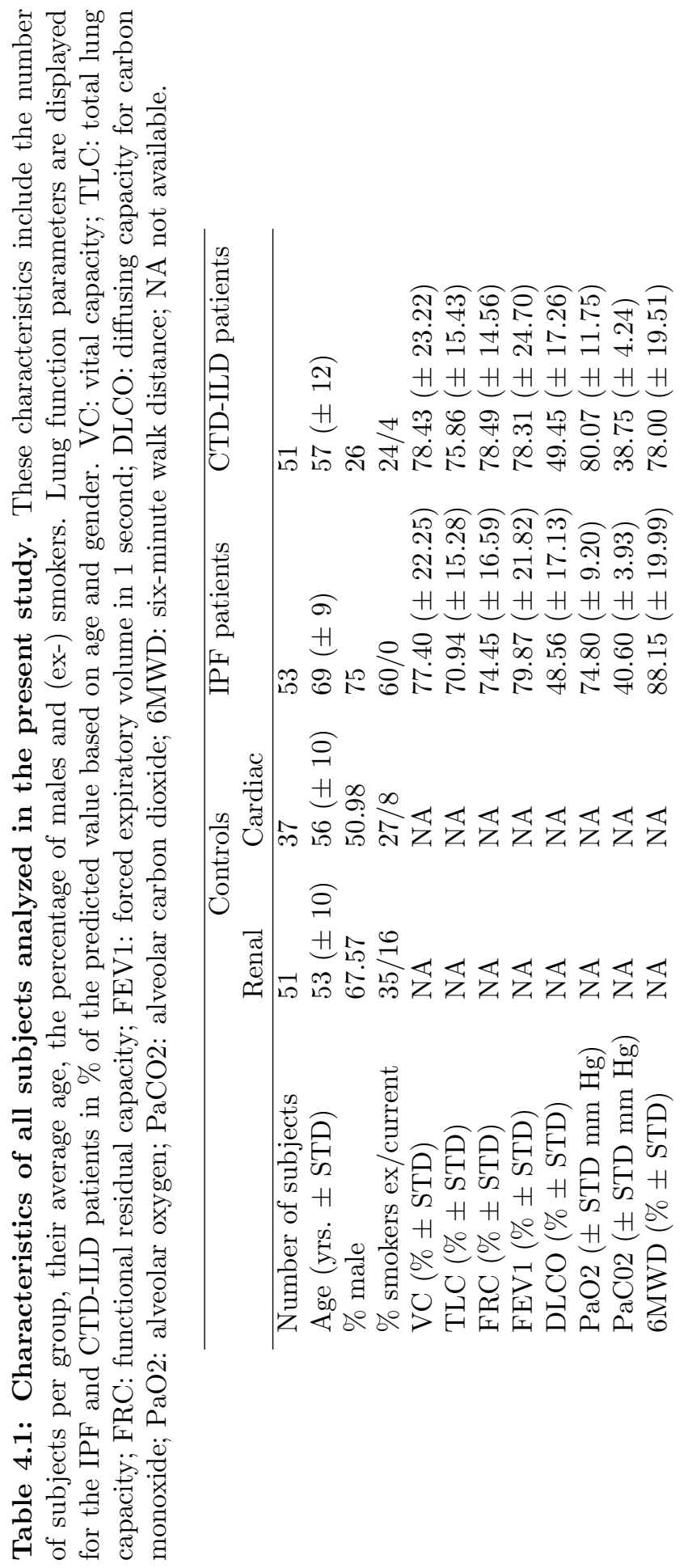




\subsubsection{Sampling and measurement of exhaled breath}

For VOCs sampling, participants were asked to sit down and breathe at a normal rate into a sterile $3 \mathrm{~L}$ Tedlar bag. The VOCs in the bag were transferred to a stainless steel two-bed desorption tube filled with carbograph 1TD/Carbopack X (Markes International, Llantrisant, Wales, UK). The desorption tubes were placed inside a TD100 automated thermal desorber for industry standard (Markes International, Llantrisant, Wales, UK) and heated to $350 \mathrm{C}$ to release the VOCs. Subsequently, $25 \%$ of the released VOCs were trapped at a cold trap at $5^{\circ} \mathrm{C}$, whereas $75 \%$ was re-stored at an identical stainless steel two-bed desorption tube for potential repeated measurement. Next, the released VOCs were injected in the GC-column at a temperature of $300^{\circ} \mathrm{C}$ and separated by capillary gas chromatography (column: RTX-5ms, $30 \mathrm{~m}$ $0.25 \mathrm{~mm} \mathrm{5 \%}$ diphenyl, 95\% dimethylsiloxane, film thickness $1 \mu \mathrm{m}$; Trace 1300GC, Thermo Fisher Scientific, Waltham, Massachusetts). The temperature of the gas chromatograph was programmed in the following manner: $40^{\circ} \mathrm{C}$ during $5 \mathrm{~min}$, then raised with $10^{\circ} \mathrm{C} / \mathrm{min}$ to a maximum temperature of $270^{\circ} \mathrm{C}$, which was maintained for 5min. Time-of-flight mass spectrometry (tof-MS; Bench TOF-dx, Almsco International, Llantrisant, Wales, UK) was used to detect and identify compounds available in the samples. Electron ionization mode was set at $70 \mathrm{eV}$ and the mass range $\mathrm{m} / \mathrm{z} 35$ 350 was measured. Sample frequency of the mass spectrometer was set to 5 scans $/ \mathrm{sec}$ and analysis run time to $33 \mathrm{~min}$. Following this procedure, a chromatogram was generated for each subject.

\subsubsection{Data processing}

After measurement, each chromatogram was processed to diminish the influence of non-biological variation. Denoising, baseline correction, alignment, normalization and scaling of the data were consecutively used based on the method previously described by Smolinska et al [27]. Briefly, log transformation of the data was performed to convert heteroscedastic noise into homoscedastic noise [32] after which the chromatograms were denoised by a Daubechies wavelet with two levels of compression [33]. Baseline correction was done by B-splines with asymmetric least squares smoothing [34] and normalization of the chromatograms was carried out by probabilistic quotient normalization [35]. Peak picking was performed after which the area under a peak was calculated. Peaks for the same compound were identified and combined using correlation of the mass spectra. This resulted in a data matrix with individual participants as rows and individual peaks as columns.

\subsubsection{Data analysis}

\subsubsection{Selection of discriminatory VOCs}

In order to apply VOCs for the identification of IPF and CTD-ILD patients, multivariate statistical modelling has been performed for selection of discriminatory VOC profiles unique for the various groups. More specifically, three comparisons were performed. First, IPF patients were compared to a group of controls sampled at the cardiac department. Second, CTD-ILD patients were compared to the group of controls 
from the cardiac department. Finally, IPF and CTD-ILD patients were compared.

The selected multivariate statistical model was Random Forests (RF) [36], a machine learning algorithm that generates a large quantity of uncorrelated decision tree predictors to classify samples into the appropriate class. This algorithm combines these decision trees to produce a generalization error called the out-of-bag error. This error demonstrates the accuracy of the model (accuracy $=1$ error). Additionally, the model provides a measure of the importance of a variable that gives the most important variables the highest value. Based on this value, a subset of variables is chosen that can discriminate between two classes, in this case patients and controls. To visualize the efficacy of the model, a principal component analysis (PCA) score plots of the RF proximities was employed. The proximities represent the similarity between individual samples as a result of the selected VOC profile: a small proximity value indicates similarity and a large proximity value dissimilarity between individual samples. Consequently, a PCA plot of proximities displays sample groupings.

\subsubsection{Internal validation}

To internally validate the distinct VOC profiles selected using RF, the out-of-bag error was calculated as described above. Additionally, the controls from the cardiac department were set aside to be used as validation of the classification model. An internal validation dataset comprises of samples measured at the same time as all other samples that are not used in the development of statistical model (in this case RF). In other words, the selection of discriminatory VOCs is not done on these samples. The internal validation step includes projecting these extra samples into the discriminatory VOC models designed for the first group of samples to predict their disease status. If successful, this internal validation step makes this discriminatory VOC profile more reliable and generalizable to a larger and independent population.

\subsubsection{Chemical identification of VOCs}

For the two comparisons between the individual diseases and the controls, a handful of the most important VOCs were selected for chemical identification. The measure of importance for each individual VOC in the two discriminatory profiles was determined using the RF-variable importance, where the VOCs with the highest value had the largest contribution to the model. The selection of VOCs for chemical identification was based on defining a cut-off point to separate the VOCs clearly standing out from the rest of the volatiles in the model. The plots displaying the RF-variable importance for the discriminatory VOCs in both models as well as the selected cutoffs are displayed in Figure 4.S2B and C. Additionally, all VOCs of the comparison between IPF patients and CTD-ILD patients were chemically identified due to their clinical relevance (see Figure 4.S2A of the supplementary material for the relative contribution of these VOCs to the discriminatory profile). The identities of these VOCs were determined in two ways: 1) spectrum recognition using the National Institute of Standard and Technology (NIST) library in combination with an in-house composed compound database in which pure compounds were previously recorded and 2) validation of the identification described in step 1 by an experienced mass spectrometrist. 


\subsubsection{Influence of confounders}

Since differences in age and gender can influence the classification between groups [37], it is important to rule out that these factors influenced the classification model in this study. The factors that were found significant between the different groups in Table 4.S1 of the supplementary material (i.e. age, gender and smoking status) were tested by regularized MANOVA [38] to determine whether they influenced the models significantly.

\subsubsection{Power calculation}

To determine the influence of the group sizes used in this study on the differences observed in discriminatory profiles, a sample size determination was performed as previously described by Blaise et al [39]. Briefly, the Cohens D effect size (i.e. a quantification of the difference of a VOC between two groups) was calculated for each discriminatory VOC after which the data are simulated by random multivariate normal simulation to find the sufficient sample size that allows finding the significant differences between cases and controls [40].

\subsubsection{Correlation between VOCs and lung function parameters}

Canonical correlation analysis (CCA) [41] was used to correlate the list of discriminatory VOCs to a list of lung function parameters. These include VC, TLC, FRC, FEV1, DLCO, PaO2, PaCO2 and 6MWD. Before CCA analysis, both datasets were log-transformed. A subset selection of the lung function parameters was made based on the contribution of the VOCs to the CCA model to achieve the best possible correlation. A correlation coefficient and corresponding p-value were reported in combination with figures of the correlation.

\subsection{Results}

To answer the research questions, the present study consists of two groups of patients (IPF and CTD-ILD) as well as two groups of controls (collected at either the renal or cardiac department). Using PCA in combination with Bhattacharyya distance [42], no clear distinction between these two control groups could be revealed. Therefore, the so-called renal control group was used to search for discriminatory VOCs between IPF or CTD-ILD patients and controls because if its larger sample size, whereas the so-called cardiac control group was set aside for (internal) validation.

Before classification, the differences in age, gender and smoking behavior in Table 4.1 were tested for significance between the three groups. No significant differences between the two control groups were found. However, the difference in age of the participants between the controls and IPF patients (p-value: 5.8-16) and between the IPF and CTD-ILD patients (p-value: 1.0-06) was significant. Additionally, the gender distribution between the controls and CTD-ILD patients (p-value: 0.002 ) and the IPF and CTD-ILD patients (p-value: 1.63-06) was significantly different. Finally, the distribution of current and ex-smokers was significantly changed between the IPF 
and CTD-ILD patients (p-value: 0.02). Nonetheless, the discriminatory VOC profiles were not influenced by these significant differences (Table 4.S2).

\subsubsection{VOC profiling for IPF patients versus controls}

The exhaled VOCs from the included 53 IPF patients were compared with those present in the breath of the 51 controls from the renal department using RF. A total of 34 VOCs was selected that could discriminate the IPF patients from the controls with $84.6 \%$ accuracy and a sensitivity of $81.1 \%$ and specificity of $88.2 \%$. The chemical identity of the 5 most important VOCs is given in Table 4.2, which shows that the concentration of all but benzaldehyde is higher in the IPF patients. The Receiver Operating Characteristic (ROC) curve has an area under the curve (AUC) of 91.2\% (Figure 4.1A). Additionally, a PCA score plot was generated to display groupings in the data as a result of the selected subset of VOCs. This score plot is depicted in Figure 4.1B and demonstrates a clear separation between both groups.
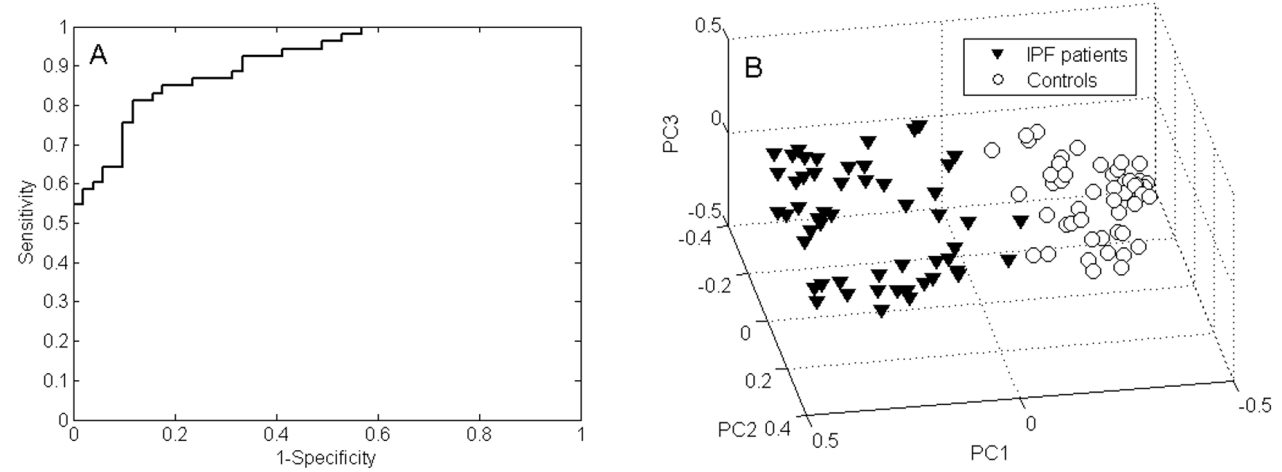

Figure 4.1: VOC profiling for IPF patients versus controls. A) ROC curve of which the AUC measures discrimination, i.e. the ability of the test to correctly classify those with and without the disease with $100 \%$ being absolute discrimination. The corresponding AUC is $91.2 \%$. B) 3D PCA plot of Random Forests proximities comparing IPF and controls. This plot is based on a subset of 34 VOCs. Each point represents an individual and therefore this plot provides an overview of all subjects and how they relate to each other. The distance between individual points expresses their similarity, i.e. short distance is highly similar and vice versa.

\subsubsection{VOC profiling for CTD-ILD patients versus controls}

When comparing the exhaled VOCs of the 51 included CTD patients with those present in the breath of the renal controls, 11 VOCs were selected as discriminatory. The 4 most important VOCs in this model are listed in Table 4.3, all of which decreased in concentration in the CTD-ILD patients compared to the healthy controls. This discriminatory VOC profile provided a classification accuracy of $77.5 \%$ with a 
sensitivity of $76.5 \%$ and a specificity of $78.4 \%$. The corresponding ROC curve and PCA scores plot are displayed in Figure 4.2A and B. The ROC AUC was $83.9 \%$.
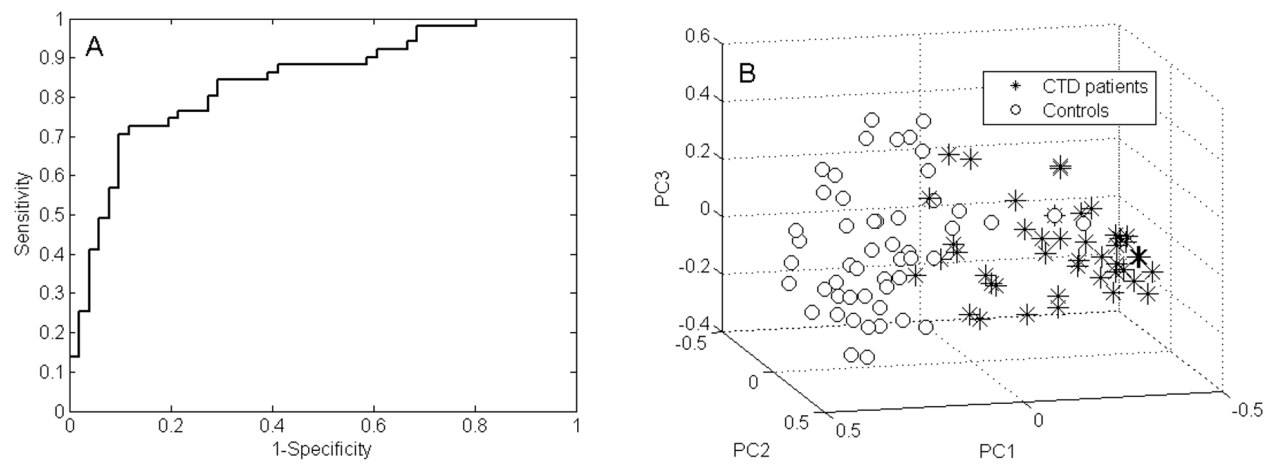

Figure 4.2: VOC profiling for CTD-ILD patients versus controls. A) ROC curve of which the AUC measures discrimination, i.e. the ability of the test to correctly classify those with and without the disease with 100B) 3D PCA plot of Random Forests proximities comparing CTD-ILD patients and controls. This plot is based on a subset of 11 VOCs. Each point in the plot symbolizes one subject in the study. The distance between two points signifies the measure of similarity, i.e. a small distance indicates high similarity and vice versa.

\subsubsection{VOC profiling for IPF patients versus CTD-ILD patients}

To accurately distinguish between the two different ILDs included in this study, i.e. IPF and CTD-ILD, the exhaled VOCs of both patients groups were analyzed and compared. A subset of 16 VOCs was able to predict both diseases with an accuracy of $76.9 \%$, a sensitivity of $75.5 \%$ and a specificity of $78.4 \%$. The ROC curve in Figure 4.3A presents an AUC of $83.8 \%$ and the PCA scores plot in Figure 4.3B displays a separation between the two patient groups. The chemical identity of all 16 VOCs is displayed in Table 4.4. Seven were exhaled in lower concentrations in the IPF patients compared to the CTD-ILD patients, whereas the other 9 revealed an opposite trend.

In order to combine the three RF models, the proximities of all three comparisons were combined to produce a PCA figure that could separate all three groups as described by Smolinska et al. [45] (Figure 4.4).

\subsubsection{Validation}

As stated in Materials and Methods section, the 37 controls from the cardiac department were excluded from the initial VOC models for validation purposes. After selecting discriminatory VOCs, the models that accurately separated both IPF and CTD-ILD patients from controls were applied to predict the class of these additional 37 controls. The accuracy of the IPF model was $56.8 \%$ and of the CTD-ILD model 

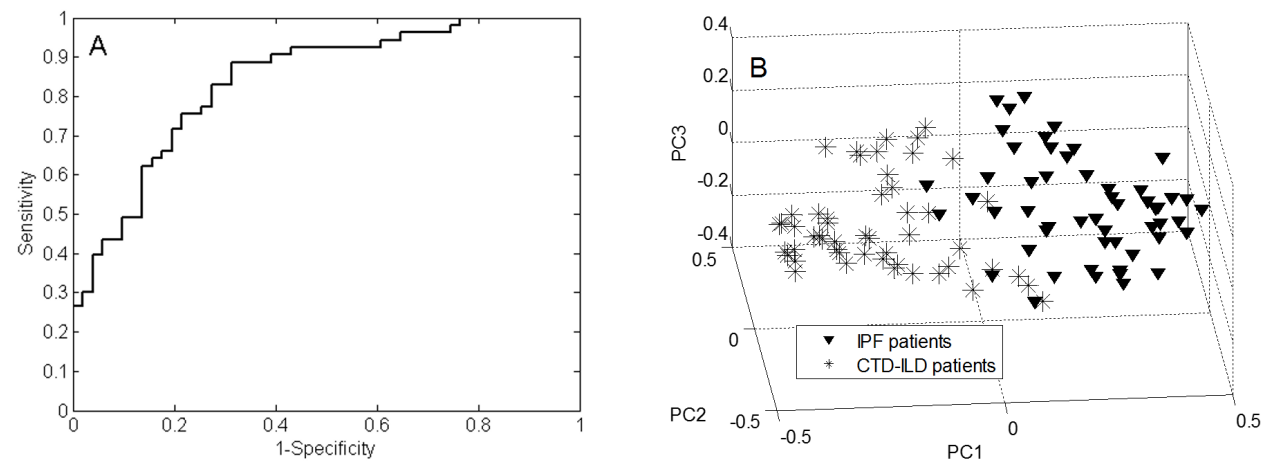

Figure 4.3: VOC profiling for IPF patients versus CTD-ILD patients. A) ROC curve and its corresponding AUC, which indicates the proportion of expected true positives/negatives by the model. B) 3D PCA plot of Random Forests proximities comparing IPF and CTD patients. This plot is based on a subset of 16 VOCs. Each point in the plot symbolizes one subject in the study. The distance between two points signifies the measure of similarity, i.e. a small distance indicates high similarity and vice versa.

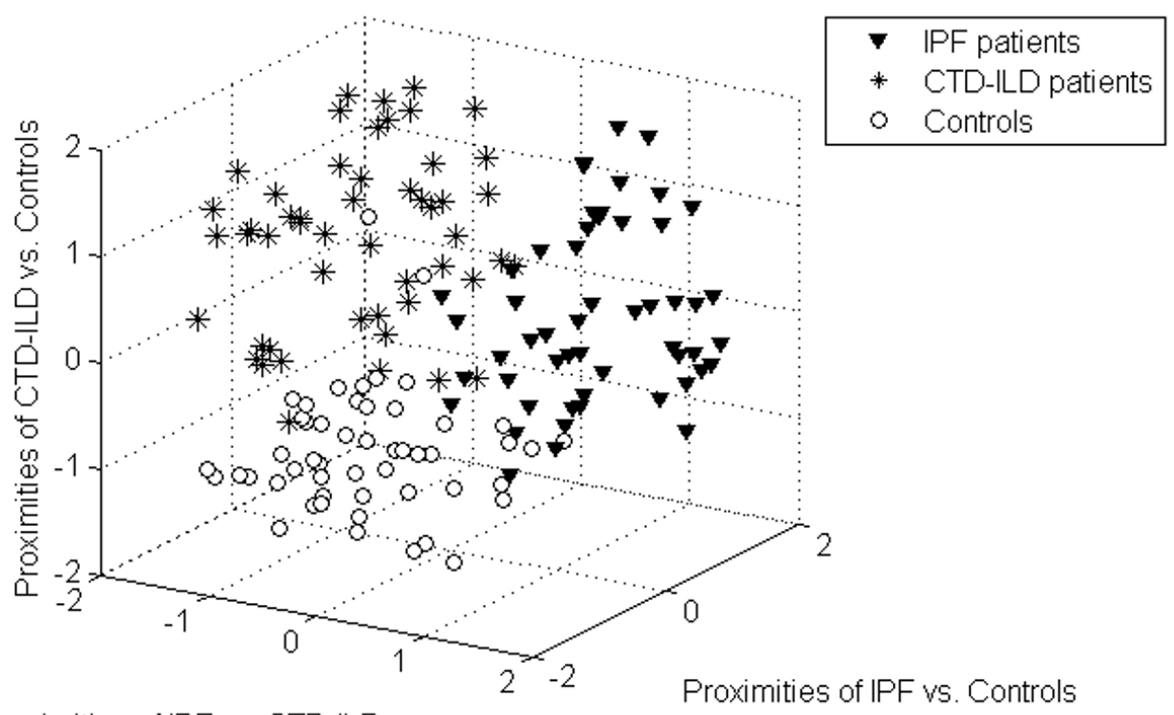

Proximities of IPF vs. CTD-ILD

Figure 4.4: VOC profiling of IPF versus CTD-ILD versus controls. 3D PCA plot on the combined Random Forests proximities. Each axis represents one comparison and each point in the plot symbolizes one subject in the study. The distance between two points signifies the measure of similarity, i.e. a small distance indicates high similarity and vice versa. 
Table 4.2: Chemical identities of the most important VOCs of the comparison between IPF and Controls

\begin{tabular}{lll}
\hline Chemical identity & CAS number & Change in IPF with respect to controls \\
\hline Ethanol & $64-17-5$ & Down \\
Heptane & $142-82-5$ & Down \\
Benzaldehyde & $100-52-7$ & Up \\
Unknown & NA & Down \\
Dimethyl sulfide & $75-18-3$ & Down
\end{tabular}

Table 4.3: Chemical identities of the most important VOCs of the comparison between CTD-ILD and Controls

\begin{tabular}{lll}
\hline Chemical identity & CAS number & Change in CTD-ILD with respect to controls \\
\hline 2-Heptanone & $110-43-0$ & Down \\
4-penten-ol & $821-09-0$ & Down \\
2,5-dimethyl furan & $625-86-5$ & Down \\
Ethanol & $64-17-5$ & Down
\end{tabular}

Table 4.4: Chemical identities of all discriminatory VOCs of the comparison between IPF and CTD-ILD

\begin{tabular}{lll}
\hline Chemical identity & CAS number & Change in IPF with respect to CTD-ILD \\
\hline Acetone & $67-64-1$ & Down \\
Dimethylsulfone & $3877-15-4$ & Up \\
Heptane & $142-82-5$ & Down \\
4-methyl-2-heptene & $3404-56-6$ & Up \\
Branched C11H24 & NA & Up \\
Undecane & $1120-21-4$ & Down \\
Tridecane & $629-50-5$ & Up \\
Octadecane & $593-45-3$ & Down \\
Branched C12H24 & NA & Up \\
Pyrrolidine & $123-75-1$ & Down \\
Decanal & $112-31-2$ & Down \\
2-heptanone & $110-43-0$ & Up \\
Branched C14H30 & NA & Up \\
4-penten-ol & $821-09-0$ & Up \\
2,5-methylfuran & $625-86-5$ & Down \\
2-thiapentane & $3877-15-4$ & Up
\end{tabular}


$46 \%$, indicating that the selected VOCs could not be validated in both models using this set of controls.

\subsubsection{Power calculation}

Because IPF and CTD are heterogeneous diseases, the group sizes applied in this study might not be large enough for the effect sizes of the VOCs that can be anticipated in such diverse patient groups. In order to test the influence of the applied power on the observed results, a power calculation was performed for all three comparisons to estimate the required sample sizes based on the effect size of each discriminatory VOC. For the VOC model that discriminates between IPF and controls, 30 of the selected 34 VOCS reached a sufficient statistical power of 0.8 when the sample size was approximately 350 samples (Figure 4.5A). For the remaining four VOCs, no sufficient sample size could be calculated to reach the required power of $>0.8$ For the VOC profile observed to distinguish between CTD-ILD and controls, all 11 VOCs reached a satisfactory power level at approximately 350 samples (Figure 4.5B). A similar trend was observed for the predictive model of IPF versus CTD-ILD where only 2 of the discriminatory 16 VOCs had insufficient effect size to reach an acceptable power (Figure 4.5C). From the remaining 14 volatiles, 1 VOC reached a power higher than 0.8 at 450 samples whereas the majority already achieved such an adequate power at approximately 300 samples.

\subsubsection{Correlation between discriminatory VOCs and lung function parameters}

To examine the clinical relevance of the identified VOCs, correlation between the discriminatory volatiles of both groups of patients and the lung function parameters characteristic for IPF and CTD-ILD was performed using CCA. As depicted in Figure ??, a significant correlation was observed between the VOCs and two of the included lung function parameters: TLC and 6MWD. A correlation coefficient of 0.8484 with a corresponding p-value of 0.0308 was achieved.

\subsection{Discussion}

As ILDs represent a rather heterogeneous group of diseases characterized by nonspecific clinical symptoms yet associated with a broad range of clinical outcomes, varying from chronic fatigue to death by respiratory failure, accurate in-time diagnosis is mandatory. Until now, invasive lung biopsies are often still required for the correct differential diagnosis in ILDs as other currently available diagnostic tools including imaging techniques (e.g. HRCT) and biological markers (e.g. chemokines, proteases and growth factors, [46] fail to be exclusive [20, 21]. Therefore, the possible usefulness of non-invasive volatile markers excreted in the breath to identify specific types of ILD has been explored in the present study.

We report an attempt to find discriminatory VOC profiles in the breath of patients suffering from IPF or CTD-ILD and healthy controls. In the present study, 

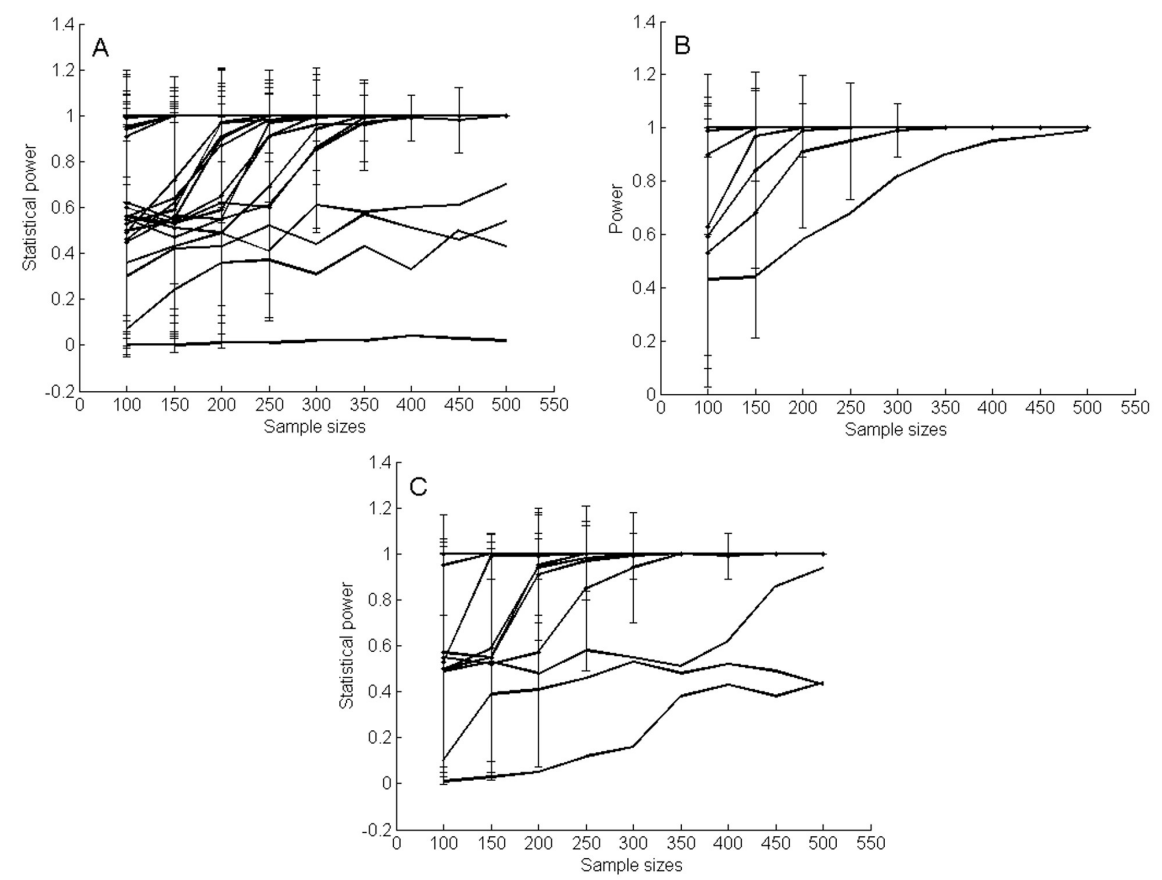

Figure 4.5: Calculated power of the discriminatory volatiles selected for each comparison for increasing sample sizes. A) Each line depicts one of the 34 discriminatory VOCs of the comparison between the IPF patients and the controls. The error bars denote the standard deviation of each line. The error bars of the lowest 4 lines were removed for clarity. B) Each line depicts one of the 11 discriminatory VOCs for the comparison between CTD-ILD and the controls. The error bars denote the standard deviation of each line. The error bars of the lowest line were removed for clarity. C) Each line depicts one of the 16 discriminatory VOCs for the comparison between IPF and CTD-ILD. The error bars denote the standard deviation of each line. The error bars of the lowest three lines were removed for clarity.

34 VOCs correctly discriminated IPF patients from healthy controls with $84.6 \%$ accuracy, whereas 11 VOCs discriminated CTD-ILD patients versus healthy controls with $77.5 \%$ accuracy. Moreover, the two ILDs were correctly distinguished from each other with an accuracy of $76.9 \%$ using a set of 16 unique VOCs. Interestingly, this last subset of 16 volatiles was strongly correlated with two clinical parameters of the diseases, i.e. total lung capacity and 6 minutes walk distance, indicating the possible pathological relevance of the selected VOCs in ILDs. Although these results are promising and could lead to potential new non-invasive markers of IPF and CDT-ILD, the set of VOCs discriminating between ILD patients and controls could not be validated in the independent control group. This additional validation, using the second control group that was put aside during the development of the initial classification model, yielded accuracies of $57 \%$ and $46 \%$ for the classification of respectively IPF 
and CTD-ILD. A possible explanation for this lack of sufficient prediction of the extra cardiac control group could be the presence of a slightly distinct breath profile when only the selected sets of discriminatory VOCs are taken into account. As described in the results section, the total VOC profiles of both control groups were compared using PCA in combination with Bhattacharyya distance to verify that they were not significantly different from each other. However, when the same analysis was performed on only the two distinct sets of discriminatory VOCs observed in this study (i.e. 34 volatiles for the separation model between controls and IPF and 11 for that between controls and CTD-ILD), the corresponding Bhattacharyya distances revealed that there are differences between these two control groups after all. The fact that these distances were very low (i.e. 0.14 and 0.16 for the 34 and 11 VOCs respectively) indicates that the differences observed between the two control groups are very low as well as the smaller the Bhattacharyya distance is, the smaller the observed differences are. Another possible explanation for this apparent lack of prediction could be the insufficient size of both the control and patient groups that might hamper capturing all possible variances induced by these heterogeneous diseases. As can be deduced from Figure 4.5, the majority of VOCs in each of the 3 discriminatory models only reached a statistical significant power when the number of individuals in each group would be 6 to 7 times higher than the current inclusion. Although, it cannot be guaranteed that with increased group sizes, the classification models would indeed have future predictive power, these sample size simulations do indicate that the number of individuals included has been the bottleneck in this study. Consequently, our results should be considered as promising pilot data and future research is warranted to explore whether the observed discriminatory VOC patterns for IPF and CTD-ILD will hold true in different and larger patient cohorts, preferably sampled at different locations. Additionally, these future studies should include other ILDs to explore if they can also be differentiated based on unique VOC profiles as well as to elucidate whether volatiles discriminative for these other ILDs overlap with those observed for IPF and CTD-ILD in the present study.

Chemical identification of the most discriminatory VOCs observed in this study lead to interesting observations. For instance, 5 volatiles have important discriminative power in more than one of the classification models. Both 2-heptanone and 4-pentan-1-ol are found in increased levels in IPF compared to CTD-ILD and display decreased relative concentrations in CTD-ILD versus healthy controls, indicating that these two VOCs might be related to CTD-ILD. Similarly, the relative concentrations of heptane are decreased in the breath of IPF patients compared to both the healthy controls and CTD-ILD patients, suggesting that this volatile is probably related to IPF pathogenesis. The remaining two VOCs that were of interest in more than one of the comparisons are dimethylsulfide, dimethylsulfone and 2,5- dimethylfuran. Dimethylsulfide is decreased in IPF compared to controls whereas the levels of dimethylsulfone, which is formed upon oxidation of dimethylsulfide by hydrogen peroxide, are increased in IPF compared to CTD-ILD. The presence of these two volatiles can be explained by enhanced production of hydrogen peroxide by NADPH oxidase 4 , activated by transforming growth factor which is considered the hallmark of IPF [43]. Finally, 2,5 dimethyl furan is a biomarker of smoking [44] and involved in singlet oxygen scavenging [45]. The fact that the levels of this volatile are decreased 
in CTD-ILD compared to controls as well as in IPF versus CTD-ILD indicates that these lower levels are not merely a reflection of the different inclusion rates of current and ex-smokers in all patients groups, but also associated with the oxidative stress underlying the pathology of ILD.

Within the IPF-specific profile, benzaldehyde levels were increased whereas the levels of ethanol, heptane and dimethyl sulfide were decreased in comparison to controls. Benzaldehyde is a naturally occurring dietary chemical present in for instance almonds, which is also used as a food additive and in scented products and cosmetics [46, 47]. Endogenously, benzaldehyde is formed out of benzylamine by semicarbazide-sensitive amine oxidase [48] which is a pro-inflammatory enzyme particularly expressed in the lungs [49] and elevated in smokers and patients suffering from inflammatory diseases [50]. Although there are no reports yet on the specific role of benzaldehyde in IPF, it has been shown in animal models that inhibition of amine oxidase reduces pulmonary inflammation and the development of fibrosis [50, 51]. Therefore, amine oxidase might be involved in IPF, causing the observed elevated levels of benzaldehyde in the exhaled breath of IPF patients. The relative concentration of heptane was reduced in the breath of IPF patients. Since heptane a known marker of oxidative stress $[55,56]$ and reported to be increased in patients suffering from various lung diseases including tuberculosis and lung cancer [52, 53], the observed decrease in IPF, a disease associated with oxidative stress as well, is remarkable and difficult to interpret.Finally, the relatively lower levels of dimethyl sulfide in the breath of IPF patients can be explained by the recent finding that this volatile offers protection against oxidative stress and ageing, two processes associated with IPF pathology, by serving as a substrate for the antioxidative enzyme called methionine sulfoxide reductase A [54].

In the breath of CTD-ILD patients, a decrease was noticed in the relative concentrations of heptanone, 4-penten-ol and 2,5-dimethylfuran compared to healthy controls. A clear explanation for the lowered levels of heptanone and 4-penten-ol is currently still lacking, although an isomer of the latter (i.e. 4-penten-2-ol) has already been reported as a marker for lung cancer [53].

The only volatile displaying the same pattern in both ILDs was ethanol, whose relative concentration was decreased in both IPF and CTD-ILD compared to healthy controls. In the human body, ethanol is constantly formed as a metabolite of acetaldehyde which is in situ generated during the metabolism of pyruvate, threonine, deoxyribose-5-phosphate and other substrates [55]. Interestingly, this endogenous formation of ethanol is under influence of various physiological circumstances and can be hampered by both ageing and oxidative stress [55], two conditions frequently reported to be associated with ILDs in general and IPF specifically [6, 7]. Interestingly, this endogenous formation of ethanol is under influence of various physiological circumstances and can be hampered by both ageing and oxidative stress [55], two conditions frequently reported to be associated with ILDs in general and IPF specifically [6, 7]. A second possible source of pulmonary ethanol secretion is the lung microbiome as a recent study of Bos et al. has revealed that bacterial DNA fragments can be linked to enzymes implicated in the production of VOCs predictive of respiratory tract colonization and/or infection including ethanol [56]. However, it has also been reported that microbiota are not disordered in ILD patients [57], suggesting that the pulmonary 
microbiome could only be involved in the altered ethanol excretion is most likely not associated with when underlying infections, are present. The observation that exhaled ethanol levels were not discriminatory between the ILDs further underlines that its excretion is not associated with infections but instead with general pathways underlying the pathology of these diseases including oxidative stress.

Most exhaled studies regarding ILDs have focused on either the fraction of exhaled nitric oxide (FENO) or markers in exhaled breath condensate (EBC). The few studies that have focused on identifying FENO in ILD display rather conflicting results [58-60], which might not be that surprising considering the fact that FENO is a marker of inflammation, a process that is nowadays no longer considered a mandatory contributor to ILD pathology [10]. Within the EBC studies performed over the last years, the focus was mostly on measuring markers of oxidative stress including malondialdehyde [61], nitrite [62], 8-isoprostane [63] and hydrogen peroxide itself [63]. However, all these markers are rather general for the occurrence of oxidative stress, a process involved in the pathology of many chronic diseases, and thus never shown to be exclusively different for specific ILDs. Moreover, these markers have always been analyzed on individual level which will obviously also hamper their usefulness to differentiate between ILDs as these multifactorial diseases cannot be characterized by a single marker or process.

Until now, only two other groups have studied the excretion of disease-specific VOCs in ILDs. In the first study in 2005, elevated level of ethane were reported to be exhaled in the breath of ILD patients [64]. Interestingly, the level of ethane was correlated with the clinical outcome parameters as well as with lactate dehydrogenase and $\mathrm{Pa}(\mathrm{O} 2)$ levels, two indicators of oxidative stress [64]. The other study measured exhaled VOCs in IPF patients and identified 5 VOCs (i.e. isoprene, ethylbenzene, p-cymene, acetoin and an unknown compound) that were significantly different in their breath compared to that of healthy controls [65]. Isoprene, ethylbenzene and p-cymene were also detected in our study, as well as m-, p-, and o-xylene as they are hard to distinguish from ethylbenzene. Their relative concentrations in our study and the corresponding p-values are depicted in Figure 4.S1 (supplementary data). None of these individual VOCs displayed a significantly altered level in the breath of IPF patients compared to that of healthy controls, although o-xylene almost reached significance (p-value 0.067). These discrepancies may arise from the fact that we employed an age-matched control group, and have measured all excreted VOCs followed by selecting discriminating VOC profiles rather than measuring a subselection of volatiles of which the significant change is analyzed on an individual level. Nevertheless, the observation of Yamada et al. that changes in VOCs are related to clinical parameters including lung function underlines the usefulness of breathomics in diagnosing IPF [65]. Similarly, we also observed a significant correlation between lung function parameters and the discriminatory VOC profiles for both ILDs. As a compromised lung function is the key clinical feature of ILDs, this observed correlation indicates that the selected VOCs may be linked to the general pathogenesis of these diseases. Future research has to elucidate whether this link is specific per ILD or general for all ILDs and whether it can be used to develop a breath biomarker of prognosis, disease severity and therapy efficacy.

In conclusion, this study reports for the first time that VOC profiles can be de- 
tected in the breath of patients suffering from IPF or CTD-ILD that differentiate them from both healthy controls and each other. Moreover, an ILD-specific VOC profile was strongly correlated with clinical parameters. Future research applying larger cohorts of patients suffering from a various type of ILDs and including external validation sets should confirm the potential use of breathomics to facilitate fast, non-invasive and proper differential diagnosis of specific ILDs in the future as first step towards personalized medicine for these complex diseases.

\subsection{Supplementary Materials}
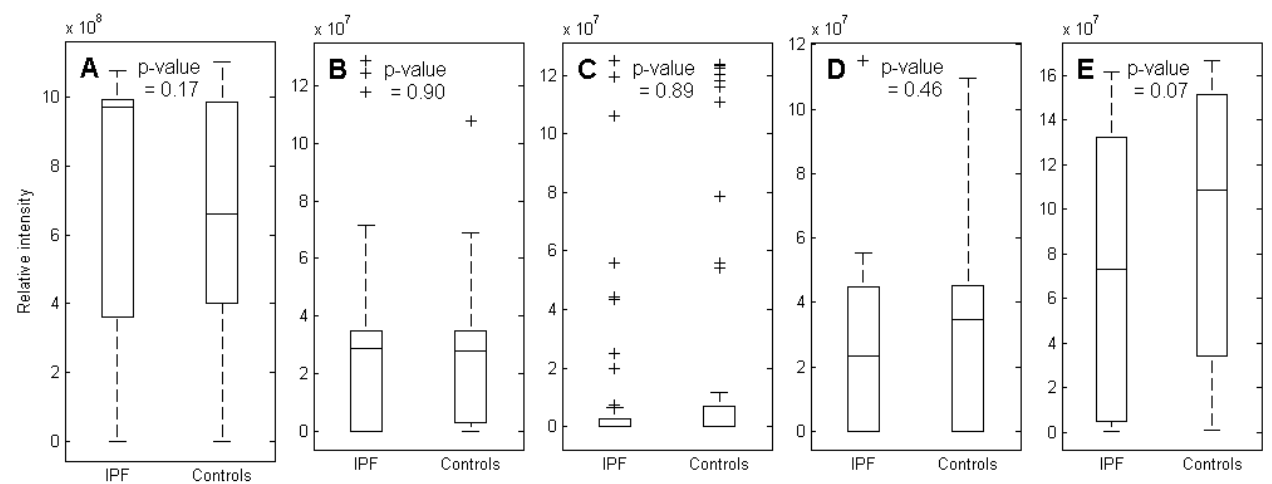

Figure 4.S1: Relative concentrations of individual VOCs reported in literature to differ in the breath of IPF patients and healthy controls. The displayed boxplots represent the following volatiles: A) Isoprene, B) p-Cymene, C) Ethylbenzene, D) m- and/or p-Xylene, E) o-Xylene. In each plot, the p-value is displayed, where a p-value ; 0.05 is considered significant. $\mathrm{m}-, \mathrm{p}-$, and o-xylene are hard to distinguish from ethylbenzene, leading to possible misidentification, thus their significances are also reported. 

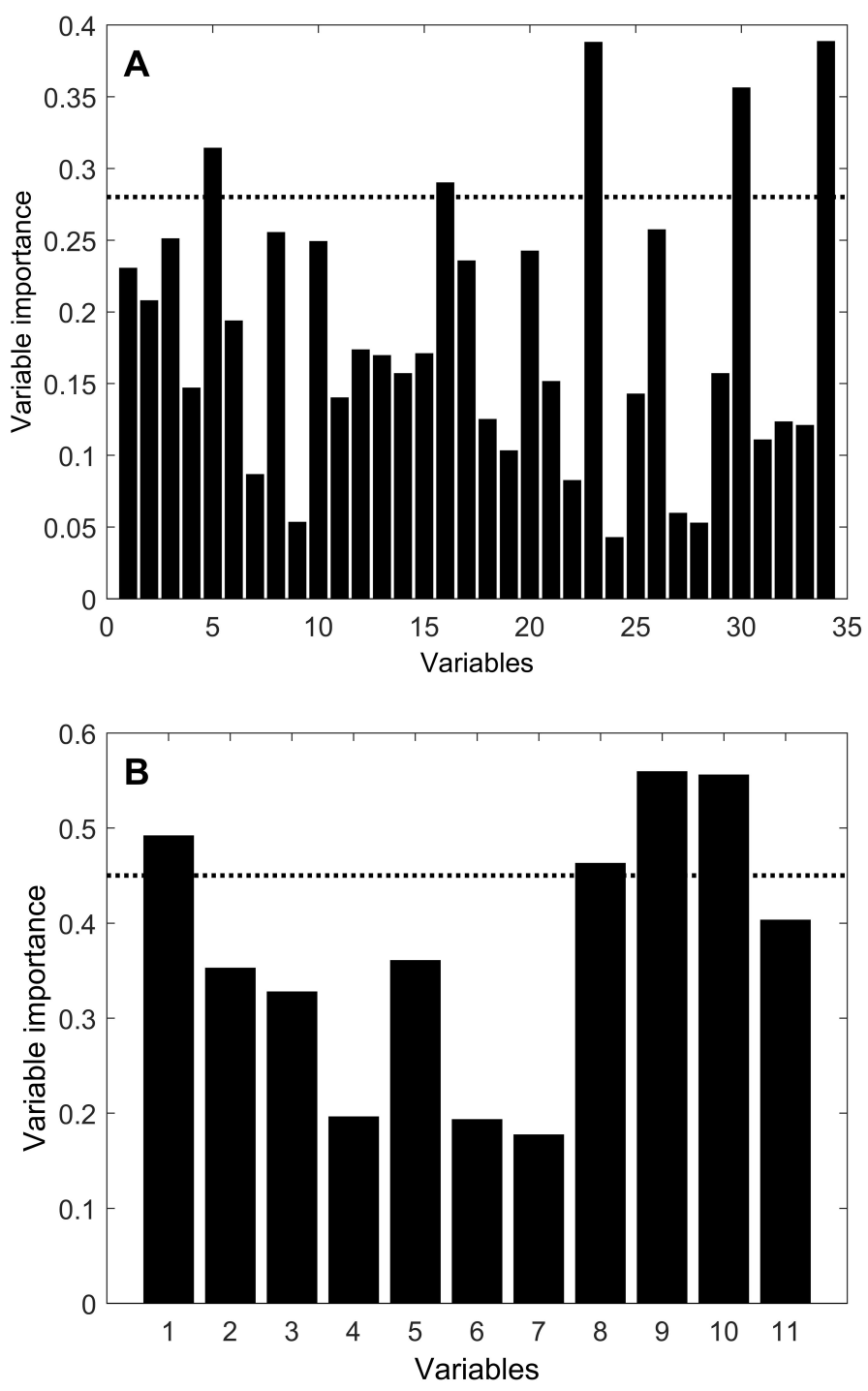

Figure 4.S2: The importance of the variables for each of the three comparisons. The dashed horizontal lines indicate the chosen cut-off to select the most important VOCs for chemical identification. A) IPF vs. controls; B) CTD vs. controls; C) IPF vs. CTD-ILD. 


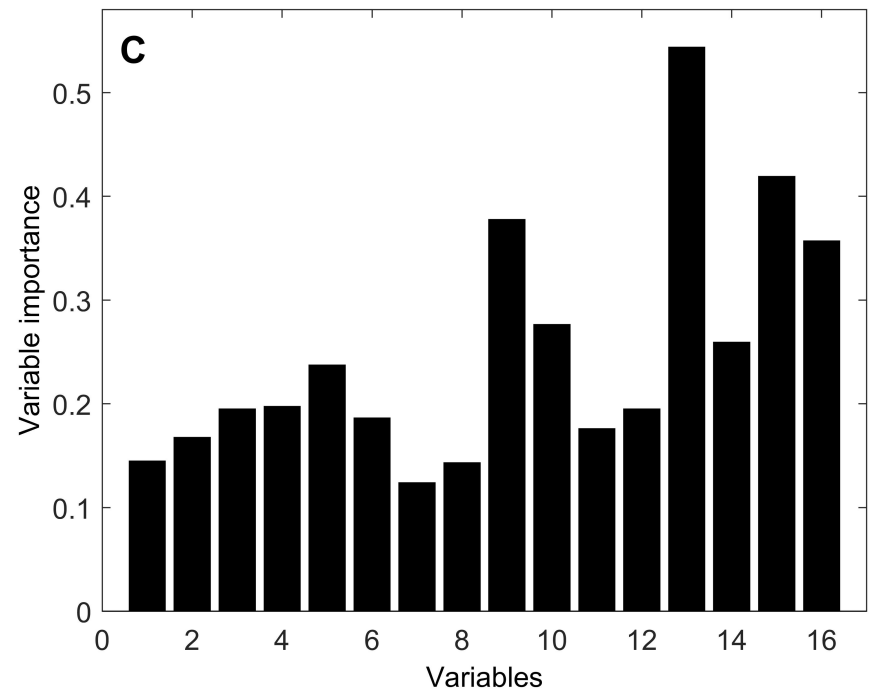

Figure 4.S3: (Continued.)

Table 4.S1: Statistical significance for the different study parameters. These significances were calculated using a Students t-test in combination with False Discovery Rate correction. *: p-value $<0.05$; ${ }^{* *}$ : p-value $<0.01$; ${ }^{* *}$ : p-value $<0.001$; ns: not significant

\begin{tabular}{|c|c|c|c|c|}
\hline & $\begin{array}{l}\text { Significance } \\
\text { renal vs. } \\
\text { cardiac } \\
\text { controls }\end{array}$ & $\begin{array}{l}\text { Significance } \\
\text { Controls } \\
\text { vs. IPF }\end{array}$ & $\begin{array}{l}\text { Significance } \\
\text { Controls } \\
\text { vs. CTD- } \\
\text { ILD }\end{array}$ & $\begin{array}{l}\text { Significance } \\
\text { IPF vs. } \\
\text { CTD- } \\
\text { ILD }\end{array}$ \\
\hline Number of subjects & ns & $* * *$ & ns & $* * *$ \\
\hline Age (yrs. old) & ns & ns & $* *$ & $* * *$ \\
\hline$\%$ male & ns & ns & ns & $*$ \\
\hline$\%$ smokers ex/current & & & & ns \\
\hline $\mathrm{VC}(\% \quad \mathrm{STD})$ & & & & ns \\
\hline TLC (\% STD) & & & & ns \\
\hline FRC (\% STD $)$ & & & & ns \\
\hline FEV1 (\% STD) & & & & ns \\
\hline DLCO (\% STD) & & & & ns \\
\hline $\mathrm{PaO} 2( \pm \mathrm{STD} \mathrm{mm} \mathrm{Hg})$ & & & & ns \\
\hline $\mathrm{PaC02}( \pm \mathrm{STD} \mathrm{mm}$ & & & & ns \\
\hline
\end{tabular}


Table 4.S2: The influence of the significant study parameters on the discriminatory VOC profiles. Regularized MANOVA was used to test whether significant study parameters were influential. A p-value $<0.05$ was considered significant.

\begin{tabular}{lll}
\hline Study Parameter & Comparison & p-value \\
\hline Age & Controls vs. IPF & 0.212 \\
Age & IPF vs. CTD-ILD & 0.219 \\
Gender & CTD-ILD vs. Controls & 0.072 \\
Gender & IPF vs. CTD-ILD & 0.814 \\
Smoking & IPF vs. CTD & 0.637
\end{tabular}




\section{Bibliography}

[1] A Fischer and S Chartrand. Assessment and management of connective tissue disease-associated interstitial lung disease. Sarcoidosis Vasc Diffuse Lung Dis, 32(1):2-21, 2015.

[2] E J Kim, H R Collard, and T E King Jr. Rheumatoid arthritis-associated interstitial lung disease: the relevance of histopathologic and radiographic pattern. Chest, 136(5):1397-1405, 2009.

[3] T A Winstone, D Assayag, P G Wilcox, et al. Predictors of mortality and progression in scleroderma-associated interstitial lung disease: a systematic review. Chest, 146(2):422-436, 2014.

[4] K M Antoniou, G A Margaritopoulos, S Tomassetti, et al. Interstitial lung disease. Eur Respir Rev, 23(131):40-54, 2014.

[5] T A Wynn. Integrating mechanisms of pulmonary fibrosis. J Exp Med, 208(7):1339-1350, 2011.

[6] S Meiners, O Eickelberg, and M Konigshoff. Hallmarks of the ageing lung. Eur Respir J, 45(3):807-827, 2015.

[7] A Bast, A R Weseler, G R Haenen, and G J den Hartog. Oxidative stress and antioxidants in interstitial lung disease. Curr Opin Pulm Med, 16(5):516-520, 2010.

[8] L Hecker, J Cheng, and V J Thannickal. Targeting NOX enzymes in pulmonary fibrosis. Cell Mol Life Sci, 69(14):2365-2371, 2012.

[9] R G Crystal, P B Bitterman, B Mossman, et al. Future research directions in idiopathic pulmonary fibrosis: summary of a National Heart, Lung, and Blood Institute working group. Am J Respir Crit Care Med, 166(2):236-246, 2002.

[10] V J Thannickal, K R Flaherty, F J Martinez, and J P Lynch 3rd. Idiopathic pulmonary fibrosis: emerging concepts on pharmacotherapy. Expert Opin Pharmacother, 5(8):1671-1686, 2004.

[11] G Raghu, H R Collard, J J Egan, et al. An Official ATS/ERSARS/ALAT Statement: Idiopathic Pulmonary Fibrosis: Evidence-based Guidelines for Diagnosis and Management. American Journal of Respiratory and Critical Care Medicine, 183(6):788-824, 2011.

[12] F Macagno, F Varone, P M Leone, et al. New treatment directions for IPF: current status of ongoing and upcoming clinical trials. Expert Rev Respir Med, 11(7):533-548, 2017.

[13] L Richeldi, R M du Bois, G Raghu, et al. Efficacy and safety of nintedanib in idiopathic pulmonary fibrosis. N Engl J Med, 370(22):2071-2082, 2014.

[14] T E King Jr., W Z Bradford, S Castro-Bernardini, et al. A phase 3 trial of pirfenidone in patients with idiopathic pulmonary fibrosis. N Engl J Med, 370(22):2083-2092, 2014. 
[15] J M Oldham, C Lee, E Valenzi, et al. Azathioprine response in patients with fibrotic connective tissue disease-associated interstitial lung disease. Respir Med, 121:117-122, 2016.

[16] Rekha Vij and Mary E Strek. Diagnosis and treatment of connective tissue disease-associated interstitial lung disease. Chest, 143(3):814-824, mar 2013.

[17] Moisés Selman and Ivette Buendía-Roldán. Immunopathology, diagnosis, and management of hypersensitivity pneumonitis. Seminars in respiratory and critical care medicine, 33(5):543554 , oct 2012 .

[18] D Khanna, S Mittoo, R Aggarwal, et al. Connective Tissue Disease-associated Interstitial Lung Diseases (CTD-ILD) - Report from OMERACT CTD-ILD Working Group. J Rheumatol, 42(11):2168-2171, 2015.

[19] T Bryson, B Sundaram, D Khanna, and E A Kazerooni. Connective tissue disease-associated interstitial pneumonia and idiopathic interstitial pneumonia: similarity and difference. Semin Ultrasound CT MR, 35(1):29-38, 2014.

[20] Philip A. Hodnett and David P. Naidich. Fibrosing Interstitial Lung Disease. A Practical HighResolution Computed Tomographybased Approach to Diagnosis and Management and a Review of the Literature. American Journal of Respiratory and Critical Care Medicine, 188(2):141-149, jul 2013.

[21] A U Wells and M A Kokosi. Subclinical Interstitial Lung Abnormalities: Toward the Early Detection of Idiopathic Pulmonary Fibrosis? Am J Respir Crit Care Med, 194(12):1445-1446, 2016.

[22] A Bango-Alvarez, M Ariza-Prota, H Torres-Rivas, et al. Transbronchial cryobiopsy in interstitial lung disease: experience in 106 cases - how to do it. ERJ Open Res, 3(1), 2017.

[23] S Pennathur, A Vivekanandan-Giri, M L Locy, et al. Oxidative Modifications of Protein Tyrosyl Residues Are Increased in Plasma of Human Subjects with Interstitial Lung Disease. Am J Respir Crit Care Med, 193(8):861-868, 2016.

[24] T M Maher. Precision medicine in idiopathic pulmonary fibrosis. Qjm-an International Journal of Medicine, 109(9):585-587, 2016.

[25] Agnes W Boots, Joep J B N van Berkel, Jan W Dallinga, et al. The versatile use of exhaled volatile organic compounds in human health and disease. Journal of Breath Research, 6(2):27108, jun 2012 .

[26] Agnes W. Boots, Lieuwe D. Bos, Marc P. van der Schee, Frederik Jan van Schooten, and Peter J. Sterk. Exhaled Molecular Fingerprinting in Diagnosis and Monitoring: Validating Volatile Promises. Trends in Molecular Medicine, 21(10):633-644, 2015.

[27] A Smolinska, A-Ch C Hauschild, R R R Fijten, et al. Current breathomicsa review on data pre-processing techniques and machine learning in metabolomics breath analysis. Journal of breath research, 8(2):027105, jun 2014.

[28] J. W. Dallinga, C. M H H T Robroeks, J. J B N Van Berkel, et al. Volatile organic compounds in exhaled breath as a diagnostic tool for asthma in children. Clinical and Experimental Allergy, 40(1):68-76, jan 2010.

[29] Ronny Schnabel, Rianne Fijten, Agnieszka Smolinska, et al. Analysis of volatile organic compounds in exhaled breath to diagnose ventilator-associated pneumonia. Scientific Reports, 5(October):17179, jan 2015 .

[30] J. J B N Van Berkel, J. W. Dallinga, G. M. Möller, et al. A profile of volatile organic compounds in breath discriminates COPD patients from controls. Respiratory Medicine, 104(4):557-563, apr 2010.

[31] Yosef Hochberg, Yoav Benjaminit, Yoav Benjamini, and Yosef Hochberg. Controlling the False Discovery Rate: A Practical and Powerful Approach to Multiple Testing. Source Journal of the Royal Statistical Society. Series B (Methodological) Journal of the Royal Statistical Society. Series B J. R. Statist. Soc. B, 57(1):289-300, 1995.

[32] G Box. Signal-to-Noise Ratios, Performance Criteria, and Transformations. Technometrics, 30(1):1-17, 1988. 
[33] B. Walczak and D.L. Massart. Wavelets in Chemistry, volume 22. Elsevier Science B.V, nov 2000.

[34] Paul H. C. Eilers. A perfect smoother. Analytical Chemistry, 75(14):3631, jul 2003.

[35] Frank Dieterle, Alfred Ross, Götz Schlotterbeck, et al. Probabilistic quotient normalization as robust method to account for dilution of complex biological mixtures. Application in1H NMR metabonomics. Analytical Chemistry, 78(13):4281-4290, jul 2006.

[36] Leo Breiman. Random forests. Mach. Learn., 45(1):5-32, oct 2001.

[37] L Blanchet, A Smolinska, A Baranska, et al. Factors that influence the volatile organic compound content in human breath. J Breath Res, 11(1):16013, feb 2017.

[38] J. Engel, L. Blanchet, B. Bloemen, et al. Regularized MANOVA (rMANOVA) in untargeted metabolomics. Analytica Chimica Acta, 899:1-12, oct 2015.

[39] Benjamin J. Blaise, Gonçalo Correia, Adrienne Tin, et al. Power Analysis and Sample Size Determination in Metabolic Phenotyping. Analytical Chemistry, 88(10):5179-5188, may 2016.

[40] Jacob Cohen and Cohen J. Statistical power analysis for the behavioral sciences. Academic Press, 1977.

[41] Bruce Thompson, Thompson, and Bruce. Canonical Correlation Analysis. In Encyclopedia of statistics in behavioral sciences. John Wiley and Sons Ltd, Chicester, UK, oct 2005.

[42] Frank J Aherne, Neil A Thacker, and Peter I Rockett. The Bhattacharyya metric as an absolute similarity measure for frequency coded data. Kybernetika, 34(4):363-368, 1998.

[43] L Hecker, R Vittal, T Jones, et al. NADPH oxidase-4 mediates myofibroblast activation and fibrogenic responses to lung injury. Nat Med, 15(9):1077-1081, 2009.

[44] J.J.B.N. Van Berkel, J.W. Dallinga, G.M. Möller, et al. Development of accurate classification method based on the analysis of volatile organic compounds from human exhaled air. Journal of Chromatography B, 861(B, 861):101-107, jan 2008.

[45] R Agarwal, S I Zaidi, M Athar, D R Bickers, and H Mukhtar. Photodynamic effects of chloroaluminum phthalocyanine tetrasulfonate are mediated by singlet oxygen: in vivo and in vitro studies utilizing hepatic microsomes as a model membrane source. Arch Biochem Biophys, 294(1):30-37, 1992.

[46] T B Adams, S M Cohen, J Doull, et al. The FEMA GRAS assessment of cinnamyl derivatives used as flavor ingredients. Food Chem Toxicol, 42(2):157-185, 2004.

[47] A Andersen. Final report on the safety assessment of benzaldehyde. Int J Toxicol, 25 Suppl $1: 11-27,2006$.

[48] Z Lin, H Li, H Luo, Y Zhang, and W Luo. Benzylamine and methylamine, substrates of semicarbazide-sensitive amine oxidase, attenuate inflammatory response induced by lipopolysaccharide. Int Immunopharmacol, 11(8):1079-1089, 2011.

[49] B Singh, T Tschernig, M van Griensven, A Fieguth, and R Pabst. Expression of vascular adhesion protein-1 in normal and inflamed mice lungs and normal human lungs. Virchows Arch, 442(5):491-495, 2003.

[50] A G Jarnicki, H Schilter, G Liu, et al. The inhibitor of semicarbazide-sensitive amine oxidase, PXS-4728A, ameliorates key features of chronic obstructive pulmonary disease in a mouse model. Br J Pharmacol, 173(22):3161-3175, 2016.

[51] F Marttila-Ichihara, K Elima, K Auvinen, et al. Amine oxidase activity regulates the development of pulmonary fibrosis. FASEB J, 31(6):2477-2491, 2017.

[52] Michael Phillips, Renee N. Cataneo, Rany Condos, et al. Volatile biomarkers of pulmonary tuberculosis in the breath. Tuberculosis, 87(1):44-52, 2007.

[53] M Phillips, N Altorki, J H Austin, et al. Detection of lung cancer using weighted digital analysis of breath biomarkers. Clin Chim Acta, 393, 2008.

[54] X L Guan, P F Wu, S Wang, et al. Dimethyl sulfide protects against oxidative stress and extends lifespan via a methionine sulfoxide reductase A-dependent catalytic mechanism. Aging Cell, 16(2):226-236, 2017. 
[55] M Ostrovsky Yu. Endogenous ethanol-its metabolic, behavioral and biomedical significance. Alcohol, 3(4):239-247, 1986.

[56] L D Bos, S Meinardi, D Blake, and K Whiteson. Bacteria in the airways of patients with cystic fibrosis are genetically capable of producing VOCs in breath. J Breath Res, 10(4):47103, 2016.

[57] Christian Garzoni, Silvio D Brugger, Weihong Qi, et al. Microbial communities in the respiratory tract of patients with interstitial lung disease. Thorax, 68(12):1150-1156, dec 2013.

[58] L Guilleminault, A Saint-Hilaire, O Favelle, et al. Can exhaled nitric oxide differentiate causes of pulmonary fibrosis? Respir Med, 107(11):1789-1796, 2013.

[59] M Malerba, A Radaeli, B Ragnoli, et al. Exhaled nitric oxide levels in systemic sclerosis with and without pulmonary involvement. Chest, 132(2):575-580, 2007.

[60] K P Tiev, J Cabane, F Aubourg, et al. Severity of scleroderma lung disease is related to alveolar concentration of nitric oxide. Eur Respir J, 30(1):26-30, 2007.

[61] M L Bartoli, F Novelli, F Costa, et al. Malondialdehyde in exhaled breath condensate as a marker of oxidative stress in different pulmonary diseases. Mediators Inflamm, 2011:891752, 2011.

[62] V Rihak, Petr Zatloukal, J Chladkova, et al. Nitrite in exhaled breath condensate as a marker of nitrossative stress in the airways of patients with asthma, COPD, and idiopathic pulmonary fibrosis. J Clin Lab Anal, 24(5):317-322, sep 2010.

[63] K Psathakis, D Mermigkis, G Papatheodorou, et al. Exhaled markers of oxidative stress in idiopathic pulmonary fibrosis. Eur J Clin Invest, 36(5):362-367, 2006.

[64] S Kanoh, H Kobayashi, and K Motoyoshi. Exhaled ethane: an in vivo biomarker of lipid peroxidation in interstitial lung diseases. Chest, 128, 2005.

[65] Yu-ichi Yamada, Gen Yamada, Mitsuo Otsuka, et al. Volatile Organic Compounds in Exhaled Breath of Idiopathic Pulmonary Fibrosis for Discrimination from Healthy Subjects. Lung, 195(2):247-254, apr 2017. 
CHAPTER 5

The necessity of external validation in exhaled breath research:

a case study of sarcoidosis

R Fijten, A Smolinska, M Drent, J Dallinga, $R$ Mostard, D Pachen, F van Schooten, A Boots

J Breath Res. 2017. Accepted for publication 


\section{$5.1 \quad$ Abstract}

As in other disciplines of omics research, reproducibility is a major problem in exhaled breath research. Many studies report discriminatory volatiles in the same disease, yet the similarity between lists of identified compounds is low. This can occur due to many factors including the lack of internal and in particular external validation. In an ideal situation, an external validation sampled at for example a different location - is always included to ensure generalization of the observed findings to a general population.

In this study, we hypothesized that sarcoidosis patients and healthy controls could be discriminated based on a group of volatile organic compounds (VOCs) in exhaled breath and that these discriminating VOCs could be validated in an external population. The first dataset consisted of 87 sarcoidosis patients and 27 healthy controls, whereas the validation dataset consisted of 25 patients and 29 controls. Using the first dataset, 9 VOCs were found that could predict sarcoidosis with $79.4 \%$ accuracy. Different types of internal and external validation were tested to assess the validity of the 9 VOCs. Of the internal validations, randomly setting aside part of the data achieved the most accurate predictions while external validation was only possible by building a new prediction model that yielded a promising yet not entirely convincing accuracy of $74 \%$ due to the indirect approach. In conclusion, the initial results of this study are very promising but, as the results of our validation set already indicated, may not be reproducible in other studies. In order to achieve a reliable diagnostic breath fingerprint for sarcoidosis we encourage other scientists to validate the presented findings.

\subsection{Introduction}

In biomedical research, biomarker discovery is of importance as it allows for the development of either diagnostic or prognostic markers of disease in the clinic [1]. These biomarkers are often derived using omics technologies from tissue, blood, urine or exhaled breath [2]. Mathematical modeling is subsequently used to identify novel biomarkers that may have diagnostic potential. However, problems occur when this modeling is not performed correctly.

To ensure reproducibility when using modeling algorithms, the so-called Transparent Reporting of a multivariate prediction model for Individual Prognosis or Diagnosis (TRIPOD) statement classifies predictive modeling studies into four types [3]. The first type is a predictive model built on one dataset and sometimes validated on that same dataset using resampling. Since this one dataset never fully represents the actual patient population, this approach leads to biased results that cannot be reproduced due to overfitting. The second type of prediction model studies uses one dataset, but sets apart a portion of the data as a so-called test or (internal) validation set. The test set is not used in the development and optimization of the model, but to validate it. This methodology is more robust but overfitting may still occur since the test set is collected within the same dataset. The third and fourth type uses an external, independent validation that measures the same outcome and parameters, 
but is sampled at a different time-point and/or hospital. The difference between type 3 and 4 is that researchers perform the validation themselves in type 3 compared to blind validation by other researchers in type 4 studies. This external, independent testing results in reliable predictions that can be reproduced by other studies.

The lack of external validation is visible in various omics fields, including genomics, proteomics and metabolomics studies, where the majority of the outcomes cannot be reproduced $[4,5]$. A similar trend is observed in the metabolomics analysis of exhaled breath, as there is little overlap in markers found in studies on the same disease. For example, two systematic reviews found a $14 \%$ and $7 \%$ overlap in discriminatory volatiles for COPD [6] and asthma [7] respectively. However, it should be kept in mind that this lack of reproducibility may also be influenced by differences in methodology as well as the complexity of exhaled breath.

Exhaled breath contains a mixture of endogenous and exogenous volatile metabolites, called volatile organic compounds (VOCs). Exogenous VOCs originate from environmental exposures, whereas endogenous VOCs are thought to be produced by microorganisms or as by-products of cellular metabolism [8]. In the lungs, most of these VOCs will diffuse from the blood into the exhaled air, while others are immediately exhaled without going into the blood [9]. When the lungs are affected by disease, cellular processes such as inflammation, oxidative stress and lipid peroxidation are altered, thereby leading to differences in either the composition or relative presence of the excreted VOCs $[10,11]$. In this study we use sarcoidosis as a research case to demonstrate the need of external validation in the field of exhaled breath. We focus on sarcoidosis because it is a multisystem granulomatous disease with a great variety in the clinical phenotype [12] presenting with symptoms such as extreme fatigue, coughing, wheezing and dyspnea $[13,14]$. The combination of these two factors makes it difficult to find a marker that can support the diagnosis of sarcoidosis sufficiently and accurately, resulting in a long route to the final diagnosis mainly by exclusion of other diseases [15].

From a clinical perspective, sarcoidosis has a prevalence of 200 and an incidence of 10 persons per 100,000 inhabitants [16]. The cause of the disease is unknown, but research suggests that sarcoidosis occurs due to a (myco)bacterial infection [17] or an interaction between immunological, genetic, environmental factors [15]. The onset is thought to occur due to an inflammatory response that causes granulomas in affected sites that are mainly found in the lungs $[14,18,19]$.

This study was designed as a TRIPOD type three study to evaluate and validate the potential of exhaled breath analysis in the diagnosis of sarcoidosis patients. The first aim of this study was to determine whether a set of VOCs could accurately distinguish between sarcoidosis patients and healthy controls. To this end, the exhaled breath of 113 participants was sampled, consisting of 87 sarcoidosis patients and 26 healthy controls. Bootstrapped Partial Least Squares was used to identify VOCs that could discriminate between the sarcoidosis patients and the healthy controls. The second aim of this study was to externally validate the findings of the first aim in an additional dataset containing 25 sarcoidosis patients and 29 healthy controls. These participants were sampled several years later using the same procedure, but at another location. We hypothesized that discrimination between sarcoidosis patients and the healthy individuals can be achieved based on exhaled VOCs analysis and that this 
can be validated in an independent external group of participants.

\subsection{Materials and Methods}

\subsubsection{General Information}

All participating patients were recruited during their regular check-ups at one of the two participating out-patient clinics. The Medical Ethical Committee of Maastricht University Medical Center (MUMC) had approved the protocol before the beginning of the study (ClinicalTrials.gov Identifiers NCT00741572 \& NCT02361281). All patients were fully informed about the aim and details of the study and have given their written informed consent.

\subsubsection{Patients}

Two clinical studies consisting of sarcoidosis patients and healthy controls (i.e. no respiratory diseases) were performed to find and validate discriminatory volatiles in exhaled air for the diagnosis of this disease. The patients were sampled several years apart at two outpatient clinics, and analyzed using different Gas Chromatography time of flight Mass Spectrometry (GC-tof-MS) instruments. The first study, designated as the discovery study consisted of 113 individuals: 87 sarcoidosis patients with varying stages of disease (see Table 5.1) and 26 healthy controls. The exhaled breath was sampled from 2010 until 2012 at the outpatient clinic in Maastricht, the Netherlands.

The second study, denoted as the validation study, contained 25 sarcoidosis patients and 29 healthy controls. All samples were collected in 2015 at Zuyderland hospital, Heerlen, the Netherlands. For an overview of the participant characteristics of both studies, see Table 5.1.

Sarcoidosis diagnosis was based on a positive biopsy in $84 \%$ of cases. In patients with typical features of Lofgrens syndrome and characteristic features of bronchoalveolar lavage (BAL) fluid analysis results, no biopsy was obtained. This policy is consistent with the World Association of Sarcoidosis and Other Granulomatous diseases (WASOG) guidelines [20]. The healthy controls included in both studies were sampled at the same location as the patients. Whenever possible, spouses were sampled; otherwise people without pulmonary disease visiting the lung department (for example accompanying a patient) were asked to participate as healthy controls.

\subsubsection{Sampling and measurement of exhaled breath}

The patients and controls were sampled during their periodic visit to their physician. Participants were asked to sit down and breathe at a normal rate into a sterile Tedlar bag with a capacity of $5 \mathrm{~L}$ (for discovery study) and $3 \mathrm{~L}$ (for validation study). The VOCs in the bag were transferred to a stainless steel two-bed desorption tube filled with carbograph 1TD/Carbopack X (Markes International, Llantrisant, Wales, UK). The samples of the discovery study were analyzed as follows: the desorption tubes were placed inside the thermal desorption unit (Markes Unity desorption unit, Markes 
Table 5.1: Overview of the participant characteristics in the discovery and validation studies. Included are the number of participants, age (mean age standard deviation), percentage of male participants, chest x-ray stage, smoking status and medication use of the participants. ${ }^{*}$ Incomplete data

\begin{tabular}{llll}
\hline Study & & Discovery cohort & Validation cohort \\
\hline \multirow{2}{*}{ No. of participants } & Patients & 87 & 25 \\
& Controls & 26 & 29 \\
Age (yrs. old) & Patients & $50 \pm 10.6$ & $53.4 \pm 11.5$ \\
Percentage of male & Controls & $50.5 \pm 10.9$ & $51.2 \pm 9.8$ \\
participants (\%) & Controls & 37 & 64 \\
& 0 & $18(21 \%)$ & 41 \\
Chest X-ray stage & I & $12(14 \%)$ & $5(20 \%)$ \\
& III & $24(28 \%)$ & $7(12 \%)$ \\
Smoking: current/ & Patients & $8 / 14 / 78$ & $3(12 \%)$ \\
ex/no (\%) & Controls & $12 / 38 / 50$ & $7(28 \%)$ \\
Medication use of & Yes & 46 & $0 / 0 / 100$ \\
patients (\%) & No & 39 & 77 \\
& Unknown & 15 & 23 \\
& & & 0
\end{tabular}

International Limited, Llantrisant, Wales, UK) and quickly heated to 350C to release the compounds from the tubes. (90\% of the released compounds were trapped on a second, identical tube for eventual repeated analysis, $10 \%$ was trapped at a cold trap at $5^{\circ} \mathrm{C}$, from where it was injected into the GC-column at a temperature of 300C. Next, VOCs were separated by capillary gas chromatography (column: RTX-5ms, 30 $\mathrm{m} 0.25 \mathrm{~mm} \mathrm{5 \%}$ diphenyl, 95\% dimethylsiloxane, film thickness $1 \mathrm{~m}$, Thermo Electron Trace GC Ultra, Thermo Electron Corporation, Waltham, USA). The temperature of the gas chromatograph was programmed as follows: $40^{\circ} \mathrm{C}$ during $5 \mathrm{~min}$, then raised with $10^{\circ} \mathrm{C} / \mathrm{min}$ until a final maximum temperature of $270^{\circ} \mathrm{C}$. In the final step this temperature was maintained for $5 \mathrm{~min}$. Time-of-flight mass spectrometry (TOF-MS) (Thermo Electron Tempus Plus time-of-flight mass spectrometer, Thermo Electron Corporation, Waltham, USA) was used to detect and identify components available in the samples. Electron ionization mode was set at $70 \mathrm{eV}$ and the mass range $\mathrm{m} / \mathrm{z} 35$ 350 was measured. Sample frequency of the mass spectrometer was set to $5 \mathrm{scans} / \mathrm{sec}$ and analysis run time to $33 \mathrm{~min}$. With this procedure a chromatogram was generated for each participant.

The samples from the validation study were measured as described above, with the following amendments: The thermal desorber used was aTD100 automated thermal desorber for industry standard (Markes International, Llantrisant, Wales, UK). The gas chromatograph used was a Trace 1300GC (Thermo Fisher Scientific, Waltham, Massachusetts) and the mass spectrometer was a bench TOF-dx (Almsco International, Llantrisant, Wales, UK). Additionally, 25\% of the sample was used for the 
actual analysis, while $75 \%$ was recollected on the same (original) sample tube. Other parameters were identical.

\subsubsection{Data processing and analysis}

\subsubsection{Data preprocessing}

Each chromatogram was first preprocessed to reduce the influence of non-biological variation. Denoising, baseline correction, alignment, normalization and scaling of the data was used sequentially based on the method described by Smolinska et al. [21]. Briefly, log transformation of the data was performed to convert heteroscedastic noise to homoscedastic noise [22], after which the chromatograms were denoised by a Daubechies wavelet with two levels of compression [23]. Baseline correction was done by B-splines with asymmetric least squares smoothing [24] and normalization of the chromatograms was carried out by probabilistic quotient normalization [25]. Peaks in a chromatogram were detected and the area under each peak was calculated. The mass spectra of the peaks at specific retention times across all samples were correlated to each other to find common peaks. This resulted in a data matrix with individual participants as rows and individual peaks as columns.

\subsubsection{Selection of discriminatory VOCs}

The discovery study was used to select VOCs that could discriminate the sarcoidosis patients from the healthy controls. Due to the imbalance of the two groups (87 patients vs. 26 controls) in this discovery set, the machine learning algorithm would most likely favor the larger over the smaller group [26]. Therefore, the number of samples in the control group was artificially increased by applying the Adaptive synthetic sampling approach for imbalanced learning (ADASYN) method [27]. This is an established technique that combats data imbalance by creating synthetic samples based on the distribution of the original samples. It functions by calculating the number of synthetic samples needed to achieve a balanced dataset and subsequently generating these synthetic samples based on the K nearest neighbors for each original sample, followed by normalization based on the data distribution, resulting in a new data matrix with synthetic samples that reside in the same space as the original samples.

A bootstrapped Partial Least Squares Discriminant Analysis (PLS-DA) model was performed on the resulting dataset to find discriminatory volatiles in exhaled breath of sarcoidosis patients and healthy controls. Bootstrapped PLS-DA is a combination of the classification and resampling techniques. The bootstrap works by resampling the data into a training ( $67 \%$ of the data) and test set (33\% of the data) many times and accumulating the classification error and compounds importance at each round. Here, a PLS-DA classification model was created 5000 times and the variable importance was calculated by means of Significance Multivariate Correlation (sMC) [28]. The training set was used to optimize the final classification model by selecting the most discriminatory compounds and number of latent variables [28]. The remaining samples in the test set were used as an internal validation of the created PLS model with the significant variables to calculate a bootstrap classification error. The final set of the discriminatory compounds was determined by selecting 
compounds that were chosen in at least 4500 of 5000 iterations. The individual statistical significance of each VOC was determined using the Wilcoxon rank sum test with subsequent Benjamini and Hochbergs false discovery rate method [29].

The predictive ability of the classification model was represented by a receiver operating characteristic (ROC) curve. For each bootstrap PLS-DA model round, the ROC and area under the curve (AUC) were calculated. The final ROC was obtained by averaging all values from separate bootstrap PLS-DA rounds. The standard deviation was visualized as a grey area around the ROC line.

\subsubsection{Identification of VOCs}

The identities of the discriminatory VOCs were determined by spectrum recognition using the National Institute of Standard and Technology (NIST) library. When the identity was not present in the NIST library, the in-house library, in which pure compounds were previously recorded, was employed for identification of the compound. In both cases, the final identity was verified by our experienced mass spectrometrist upon spectrum interpretation.

\subsubsection{External Validation of the findings}

The validation study was used as an external independent validation of the PLSDA model with the subset of discriminatory VOCs derived from the discovery study. First, the peaks of the discriminatory VOCs were identified and extracted in each chromatogram. Two approaches were then used to assess the validity of the model:

1. Prediction of the validation study samples using the PLS-DA model built in the discovery study. The sensitivity and specificity were then calculated as a measure of accuracy of the classification model.

2. Creating a new PLS-DA model for the validation study with the optimized model parameters (i.e. latent variables and discriminatory VOCs) from discovery set. The model created for the discovery dataset was not used. The measure of accuracy was assessed by means of sensitivity and specificity.

\subsection{Results}

The exhaled breath of each participant of one of the two studies was collected and analyzed by GC-tof-MS to produce a chromatogram that, after pre-processing, contained 430 different VOCs matched across multiple samples.

\subsubsection{Participant characteristics}

The characteristics of the participants in both studies are demonstrated in Table 5.1. No significant differences in the characteristics between different groups of participants were identified. The patient group contained a higher percentage of men $(57 \%$ and $64 \%$ vs. $38 \%$ and $41 \%$ male versus female for respectively the discovery and validation study; not significant). The distribution of the chest X-ray stages was similar in both 
cohorts. Finally, 10 smokers were included in the discovery study, whereas none were present in the validation study.

\subsubsection{Selection of discriminatory VOCs}

First, the discovery study was used to find VOCs that could discriminate between the patients and controls. Hence, a bootstrapped PLS-DA model was used to filter these VOCs. Based on this approach, a group of 9 VOCs was identified as discriminatory. The resulting classification model discriminated between patients and controls in $79.4 \% 3.8$ of cases, with a sensitivity and specificity of $75.4 \% \pm 4.6$ and $92.5 \% \pm$ 5.0 , respectively. The corresponding ROC curve had an area under the curve (AUC) of $91.3 \% \pm 2.7$ and is depicted in Figure 5.1(a) including the overall standard deviation indicated by the grey area above and below the line. Figure 5.1(b) depicts the PLS-DA scores plot and demonstrates that the control samples were clustered together whereas the patients were more spread out. Additionally, to ensure smoking behavior did not influence the selection of discriminatory VOCs, the model was recreated after removing the 10 current smokers included in the discovery study. The exclusion of those 10 participants reduced the predictive capacity by $2.2 \%$ due to the lower number of samples in the model. Since these findings suggested that smoking did not influence the model, all samples were included to achieve the highest possible prediction.
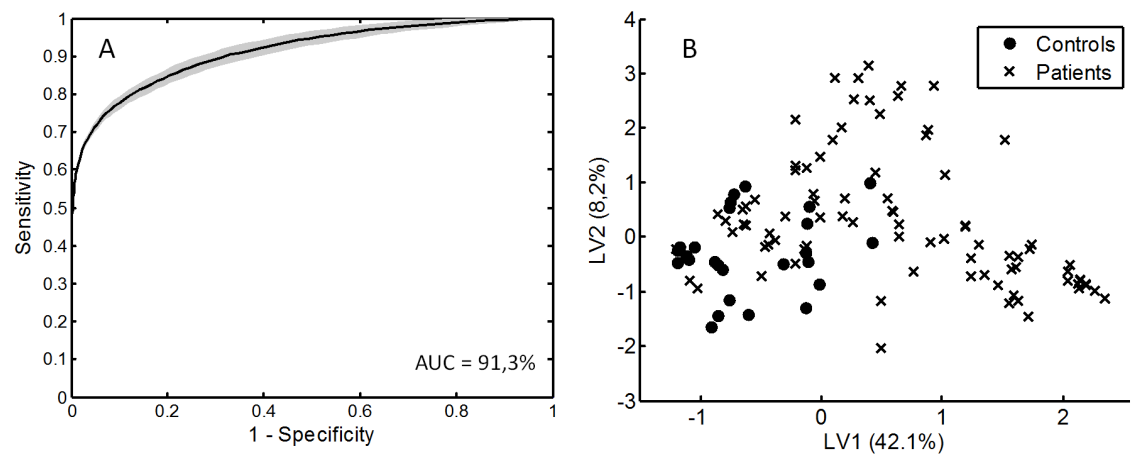

Figure 5.1: Results of the bootstrapped PLS-DA model. (a) Averaged ROC curve over all bootstrap iterations of discovery study. 1-specificity is depicted on the $\mathrm{x}$-axis, sensitivity on the $\mathrm{y}$-axis and the area under the curve (AUC) is $91.3 \%$. The standard deviation of the ROC curve is given as the grey area around the black line. (b) Score plot of the PLS-DA model built on the discovery study with 9 VOCs. Circles $=$ healthy controls, crosses $=$ sarcoidosis patients. The percentages of explained variance of $\mathrm{Y}$ of both latent variables (LVs) are mentioned between the brackets.

Table 5.2 shows the chemical identity of the 9 discriminatory VOCs and their CAS number. The importance of each VOC in the model is visualized in Figure 5.2. It also demonstrates that all VOCs except iodomethylcyclopentane were exhaled in a lower concentration by the patients than the controls. Isoprene, 3-methylhexane 
and 2-methylpentane were the most important contributors to this model whereas benzene was the least important VOC. The statistical significance of the individual VOCs in the discriminatory set is indicated in Figure 5.2. Interestingly, all VOCs except dibenzofuran and benzene were individually significant between the sarcoidosis patients and controls.

Table 5.2: Discriminatory VOCs between sarcoidosis patients and controls. The compound name and CAS numbers are displayed

\begin{tabular}{lll}
\hline VOC & Identity & CAS Number \\
\hline 1 & Isoprene & $78-79-5$ \\
2 & 2-methylpentane & $107-83-5$ \\
3 & Benzene & $71-43-2$ \\
4 & 3-methylhexane & $589-34-4$ \\
5 & p-Benzoquinone & $106-51-4$ \\
6 & Phenol & $108-95-2$ \\
7 & D-limonene & $5989-27-5$ \\
8 & iodomethylcyclopentane & $27935-87-1$ \\
9 & Dibenzofuran & $132-64-9$
\end{tabular}

\subsubsection{Internal and external validation of the discriminatory VOCs}

In order to assess the validity of the VOCs identified in the discovery study, different types of validation were used to assess the predictive capacity of the model. We performed two types of internal validation, namely leave-k-out cross-validation and randomly setting aside a percentage of the data, and compared them with external validation on a separate validation study. The external validation was done by predicting the samples of the validation study with the PLS-DA model based on the discovery study. The prediction scores for all different validation methods are demonstrated in figure 5.3, which demonstrates that leave-k-out cross-validation results in an overestimated prediction and thus insufficiently reflects the actual prediction value of the external validation. Interestingly, the prediction scores of a randomly set aside test set did reflect the external validation score well. Additionally, increasing the number of samples set aside for the internal validation in both methods applied did not influence their prediction scores yet reduced the standard deviation of these predictions.

\subsubsection{Indirect external validation}

As can be deducted from figure 5.3, the direct prediction of the validation study by means of PLS-DA model was not successful as the prediction was 53.7These results indicate that a possible batch effect between the two studies was interfering with the predictive capacity of the model. This could be due to many factors such as instrument differences, sample location, time. Batch effect correction was attempted 


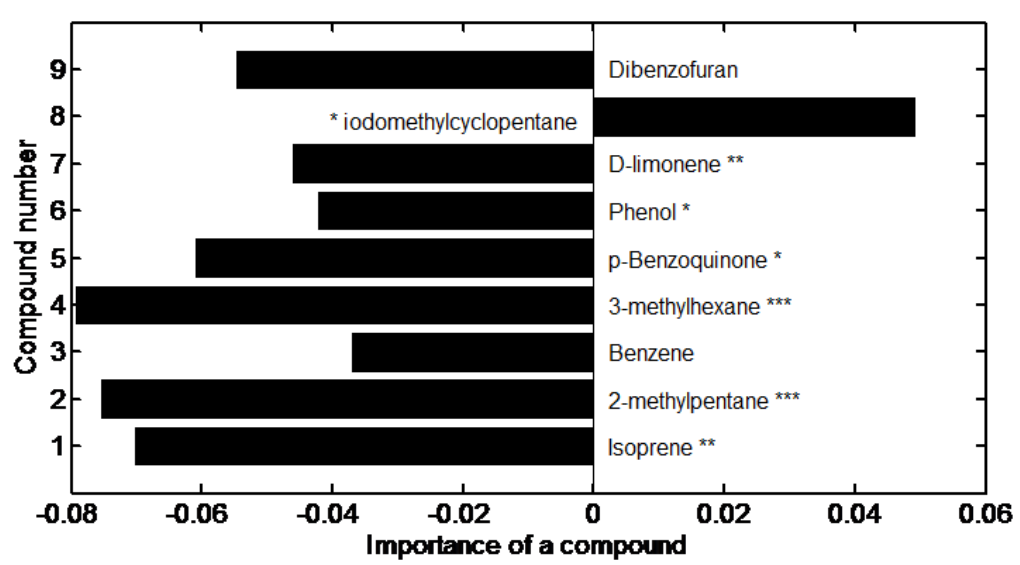

Figure 5.2: Relative importance of the 9 discriminatory VOCs in the classification model. The length of the bar indicates the relative importance of that specific VOC. The direction of the bar (positive of negative value) indicates whether a compound is elevated (positive value) or decreased (negative value) in the patients compared to the controls. Statistical significance was calculated with the Wilcoxon ranksum test, and was corrected using Benjamini and Hochbergs false discovery rate method [29]. It is indicated in the figure in this fashion: $\mathrm{p}<0.05^{*}, \mathrm{p}<0.01^{* *}, \mathrm{p}$ $<0.001 * * *$

using mean centering, log transformation and moving median correction [30], but these were not sufficient (data not shown).

\subsubsection{New model for validation study}

In order to assess the validity of the 9 selected VOCs, a separate PLS-DA model was created on the validation samples. The resulting classification model had an overall prediction of $74.1 \%$ and a sensitivity and specificity of $68 \%$ and $79.3 \%$, respectively. The corresponding ROC curve had an AUC of 76.4\% and is shown in Figure 5.4(a). In Figure 5.4(b) the classification values of the samples from the validation study are shown. A positive value indicates an allocation of the sample to the patient class, whereas a negative value indicates an allocation of the sample to the control class. The closer the number is to 1 or -1 the more certainty the classification model has in predicting a sample. The results here indicate that the classification model has low confidence in allocating the samples to sarcoidosis patients and controls groups. Therefore, many values are centered around 0 irrespective of their class.

\subsubsection{Comparison of cross-validation methods with external validation}

To demonstrate the necessity of external validation, we performed two types of internal validation on the discovery dataset, namely leave-k-out cross-validation and 


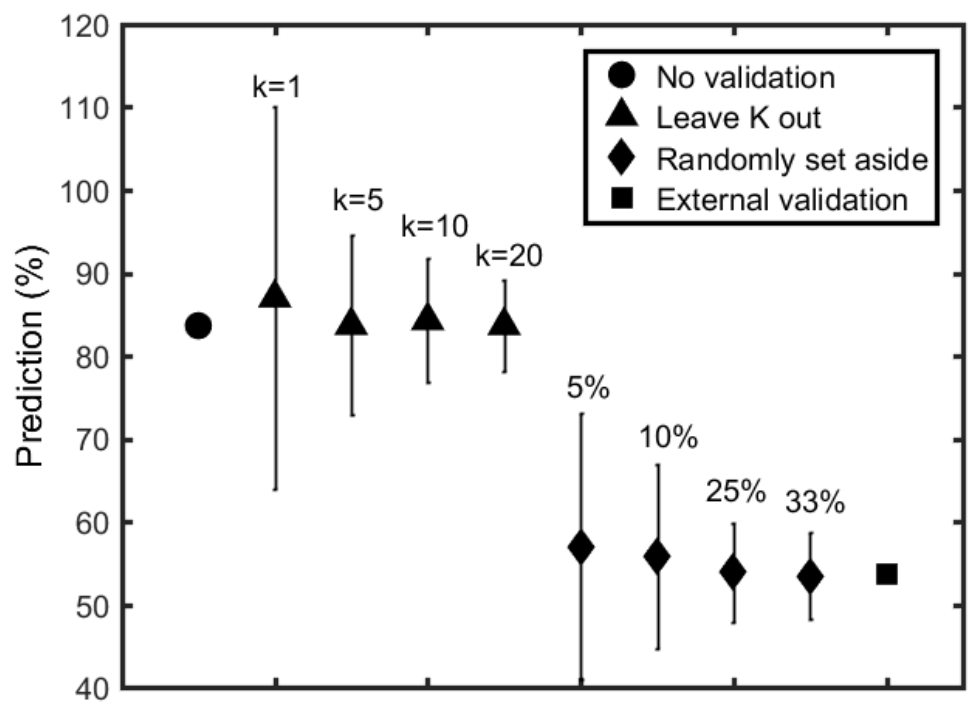

Figure 5.3: Comparison of predictions of a model on an internal or external validation dataset. It includes the model without validation (circle), with leave-kout (triangle) and randomly setting aside a test set (diamond). External validation was used as a reference (square). The ks above the triangles denote the number of samples used for leave-k-out cross validation. The percentages above the diamonds stand for the percentage of samples that were randomly set aside from the discovery dataset

randomly setting aside a percentage of the data, and compared them with the external validation performed as described above. The prediction scores for all different validation methods are demonstrated in Figure 5.5, which clearly demonstrates that leave-k-out cross-validation results in an overestimated prediction and thus insufficiently reflects the actual prediction value of the external validation. Interestingly, the prediction scores of a randomly set aside test set did reflect the external validation score well. Additionally, increasing the number of samples set aside for the internal validation in both methods applied did not influence their prediction scores yet reduced the standard deviation of these predictions.

\subsection{Discussion}

The majority of exhaled breath studies focuses on identification of biomarkers to detect a specific disease such as lung cancer [9], COPD [6], and asthma [31]. Although these breath studies demonstrate promising results, a major problem is their lack of reproducibility as they show little overlap in the selected disease-specific mark- 

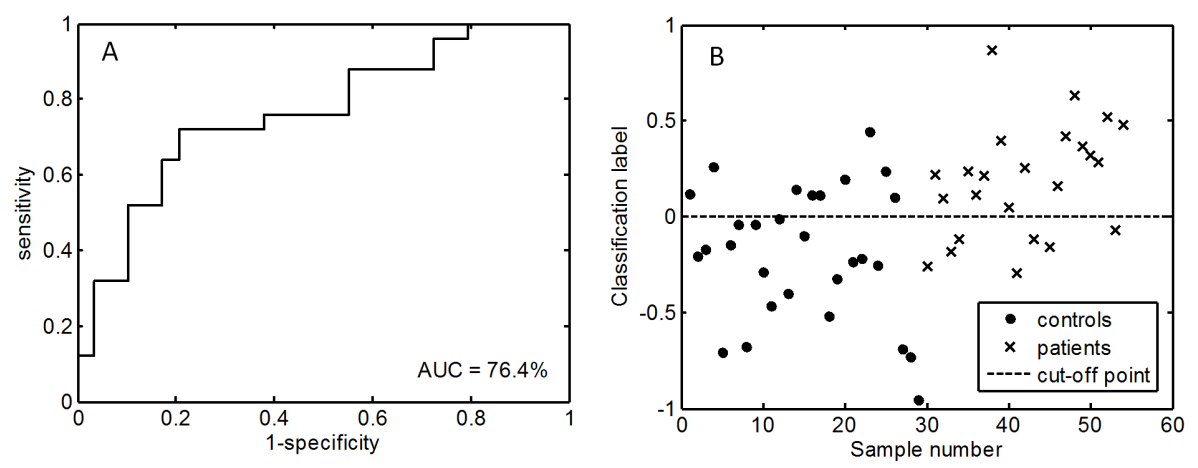

Figure 5.4: ROC curve and score plot of the PLS-DA model on the validation study with 9 discriminatory VOCs. (a) ROC curve of the validation study based on the 9 selected VOCs by the PLS-DA model built on the discovery study. The area under the curve (AUC) of the ROC curve is $76.4 \%$. (b) Classification values for each sample of the validation study. The PLS-DA model gives each sample a classification value. A cut-off of 0 is generally selected where samples with negative classification values are classified as controls, and samples with a positive value are classified as patients. Circles $=$ controls, crosses $=$ patients, dashed line $=$ cut-off.

ers $[6,7]$. Similarly, in other omics fields, demands for reproducible results have been raised in recent years after a majority of omics results could not be verified [32]. This lack of reproducibility may be due to many factors, such as low statistical power, poor quality control, introduction of false positives, overfitting and the absence of external validation [33]. The inclusion of an independent external validation study enables researchers to test if their findings are applicable to the general patient population. Therefore, this study not only attempted to find VOCs that could discriminate between sarcoidosis patients and controls using a discovery study, but also to subsequently validate those findings in an external validation dataset. This way it would ensure that the findings were reproducible in the general sarcoidosis patient population.

We found a set of 9 VOCs that could differentiate between 87 sarcoidosis patients and 26 healthy controls. A validation dataset consisting of 25 patients and 29 healthy controls was used to test the reproducibility of these 9 VOCs. To our knowledge only one other exhaled breath study using GC-MS reported inclusion of a validation study [34], which reported a decline in accuracy from $74 \%$ to an average of $69 \%$ sensitivity and from $70.7 \%$ to an average of $68.2 \%$ specificity. This decline in accuracy is similar to the ones reported in the current study. However, one important difference between our study and the one performed by Phillips et al. is that their validation dataset was sampled at the same hospitals as the original dataset and it is unclear if there was a difference in time of sampling. Since they were able to project their model onto the validation dataset, which was not the case in the current study, the difference in setup suggests that a large amount of the variation observed in our study is introduced by sampling at different hospitals during different time periods. 


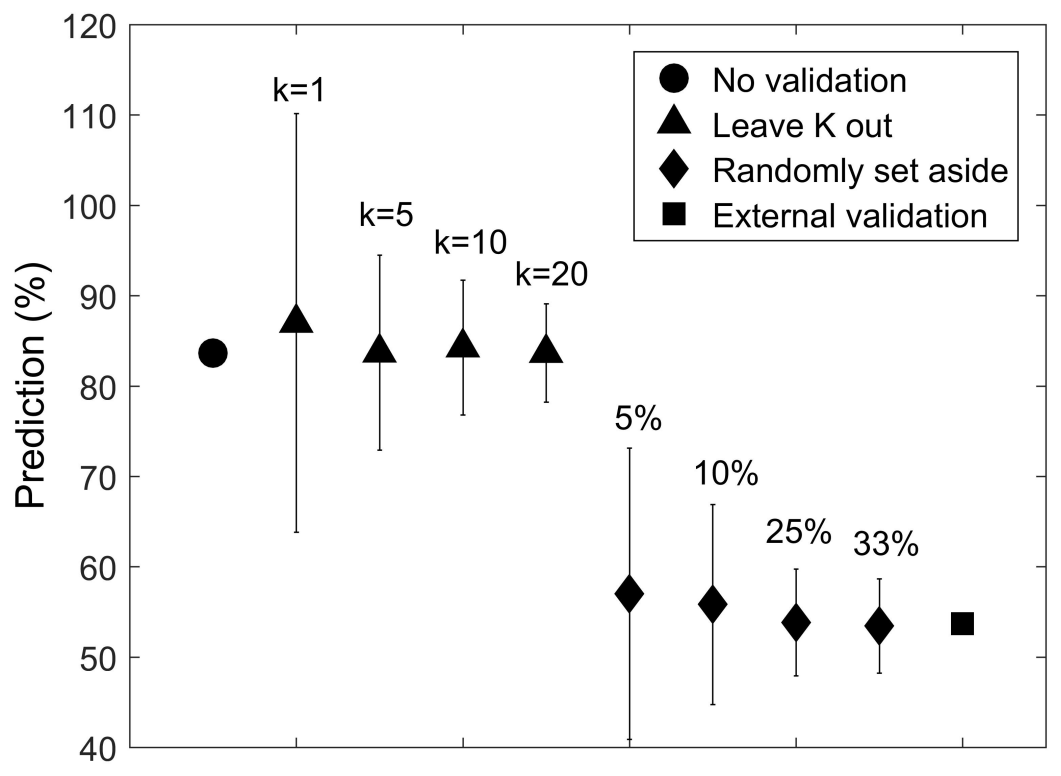

Figure 5.5: Comparison of predictions of a model on an internal or external validation dataset. It includes the model without validation (circle), with leave-kout (triangle) and randomly setting aside a test set (diamond). External validation was used as a reference (square). The ks above the red dots denote the number of samples used for leave-k-out cross validation. The percentages above the black dots stand for the percentage of samples that were randomly set aside from the discovery dataset.

Additionally, a handful meta-analyses have been performed that tried to combine data from separate studies $[6,7]$. However, they report little overlap in the identified VOCs, which indicates that the individual studies are not reproducible. Several problems have been identified in these studies. For example, by calculating p-values for each peak, with or without multiple testing corrections, or applying insufficient statistical validation by means of internal cross-validation methods $[35,36]$, the results of these studies are not applicable to a wider patient population due to large margins of false positives or overfitting of a model [3].Research has shown that internal cross-validation like leave-one-out and leave-k-out cross-validation overestimates the true predictive capacity of a model [36], with leave-one-out cross-validation as one of the most incomplete measure of predictive power. Other internal validation methods like bootstrapping (where parts of the data are randomly set aside with replacement) or jackknifing are more stable and give a more accurate prediction error [36]. Our findings support the statement that leave-one-out and leave-k-out cross-validation drastically overestimate the true performance of the classification model. Setting 
aside a random subset of individuals as an internal validation, as done previously by Phillips et al. [34], does give a much better view of what the real prediction power of the selected markers is, indicating that this method of internal validation is useful and the most suitable when external validation cannot be applied. It is interesting to note that when more samples are set aside, the standard deviation of the prediction accuracy decreases, resulting in a model that is more certain of its predictive capacity. Although setting aside a subset of individuals yields a good estimation of the prediction error, an independent external validation at a different clinic will still be the most reliable method to test the predictive power of the classification model and will increase the reliability of the results [37]. These conclusions are in line with a comparison of classification methods in breath analysis by electronic noses [38]. These authors found in a literature review that only 7 out of 46 articles applied external validation in an independent dataset. After re-analysis of available raw data of 4 studies they found inconsistent results. They concluded that an external validation cohort extracted from a related population that is separated from the training cohort by either time or place, should always be included to estimate the true diagnostic performance [38].

Although a direct prediction of the validation samples by means of the selected set of discriminatory 9 VOCs was not successful, a separate model performed on the validation dataset was promising yet not entirely convincing. This may arise from various factors including the heterogeneity of the patient population, differences in sampling locations, mechanical variation due to different GC-tof-MS machines, and procedures used to acquire chromatograms. Inter-laboratory reproducibility has not been tackled by the exhaled breath community, but poses a significant challenge ahead that may contribute to the lack of overlap in identified VOCs in scientific literature. Still, in order to apply breathomics in a clinical setting throughout the world, it is important that exhaled breath analysis (including external validation) works in heterogeneous patient groups across different locations and analytical platforms. Nonetheless, the validation model of the subset of 9 VOCs yielded a prediction of $74 \%$, suggesting that the VOCs found in the discovery dataset do have some value. Yet, more research is needed to properly validate these 9 VOCs.

This study has several limitations. First, the discovery dataset was imbalanced with respect to participant numbers in the two groups, since 87 patients but only 26 controls were included. These imbalanced numbers resulted from the strategy to including spouses as controls to minimize the influence of dietary and environmental exposures on the measured VOC profiles. However, the majority of spouses could not participate due to their own compromised health status. Additionally, adding other controls that were not matched for these factors would have introduced subgroupings in the data, further complicating the data analysis. The ADASYN method [27] was employed to correct for the imbalance. Second, even though a new model was created on the validation study for the 9 selected VOCs, the number of samples was too low to definitively validate the findings. This may be due to the large heterogeneity of sarcoidosis patients [18]. To enhance the reliability and counteract this heterogeneity, a larger number of samples is required. Additionally, phenol was detected as a discriminatory volatile even though it is always present in the Tedlar bags themselves [3941]. However, considering the fact that endogenous phenol can add to this continuous level 
and that two other members (benzene and p-benzoquinone) of the same metabolic pathway were found, it is likely that the described benzene metabolism pathway, and therefore phenol, does play a role [39]. Still the the discriminatory power of this particular VOCs should be treated with caution. Finally, the most important limitation of this study is that there are several differences between the discovery and validation datasets (location, time and GC-tof-MS machines used), making it difficult to determine why the prediction was not conclusive. From the set of discriminatory VOCs, the majority belongs to the groups of linear and branched hydrocarbons that have been found in breath of healthy as well as diseased individuals [10, 40]. Isoprene, phenol and p-benzoquinone can be mostly related to endogenous processes occurring in human body. The role of the remaining compounds remains elusive and therefore plausible explanations for their origin cannot be offered. However, an attempt has been made to provide the potential endogenous or exogenous source of each VOC.

\subsubsection{Isoprene}

Isoprene is produced in the human body as a by-product of the mevalonate pathway, which produces cholesterol and vitamin K [41]. Second, several studies have been performed that indicate a relationship between isoprene levels in exhaled breath and exercise $[42,42]$. Studies on lung diseases reported that decreased exhaled isoprene levels are potentially linked to inactivity [43], making it plausible that the decreased isoprene levels observed in sarcoidosis patients in this study are due to inactivity associated with fatigue $[13,44]$. Finally, the Streptomyces genus can produce this VOC through the non-mevalonate pathway [45]. Streptomyces is part of the normal lung microbiome, which may be disrupted in sarcoidosis patients [46]. It generally causes infection in immunocompromised patients, which is also the case in sarcoidosis $[47]$.

\subsubsection{Limonene}

Limonene is a hydrocarbon that can occur as two enantiomers: D-limonene and Llimonene [48]. The GC-tof-MS used in this study cannot distinguish between the two enantiomers, so it is unsure which one was detected. D-limonene is the naturally occurring limonene found in citrus-fruits whereas L-limonene is found in a variety of trees and herbs [49]. Since D-limonene is more likely to be found in humans, we mentioned this isomer in Table 5.2 and Figure 5.2. In this study, limonene was exhaled in a reduced concentration by sarcoidosis patients compared to the healthy controls. Because D- and L-limonene can be degraded by a variety of bacteria (in addition to fungi and yeasts), an altered lung microbiome in sarcoidosis patients may result in a reduced level of exhaled limonene.

\subsubsection{Benzene, phenol and p-benzoquinone}

Benzene is considered a pollutant that mainly originates from smoking and vehicle exhausts [50], and indoor cooking, attached garages and heating [51]. When it enters the body, it is metabolized into benzene oxide, which is then converted into 
benzene dihydriol or undergoes nonenzymatic rearrangement into phenol. Phenol is then converted to hydroquinone that is subsequently converted to p-benzoquinone and back [39].

Benzene, phenol and p-benzoquinone were all found in reduced concentrations in patients versus healthy controls. These findings suggest that patients were either exposed to less benzene or metabolize it more quickly into benzene dihydrodiol, instead of phenol and subsequently p-benzoquinone. Due to sarcoidosis-associated fatigue it is expected that the patients spend more time indoors than controls [13]. However, benzene concentrations are generally similar or higher compared to outdoor concentrations. For instance, a ratio of 1.2 and 1.4 between indoor and outdoor benzene concentrations were found in two German cities [52]. In Finland, the outdoor benzene concentration was $1.51 \mathrm{~g} / \mathrm{m} 3$, compared to $1.87 \mathrm{~g} / \mathrm{m} 3$ indoors [53]. Considering this conflicting evidence, it is unclear why lower benzene in the exhaled breath was observed in patients. It is important to note that phenol is excreted by Tedlar bags [54-56], and therefore part of the phenol measured in the samples was due to this exogenous source. It is unclear how much of the measured phenol was due to this exogenous source, so the discriminatory power of phenol needs to be interpreted with caution.

\subsubsection{Branched alkanes}

The branched alkanes 2-methylpentane and 3-methylhexane were both significantly lower in concentration in patients versus healthy controls and were previously reported [40]. Both VOCs are considered pollutants [57, 58]], but it is also hypothesized that these compounds are produced due to alterations in oxidative stress levels [59], [60]]. However, since oxidative stress is increased in sarcoidosis [61] higher concentrations of branched alkanes were expected in the patients compared to the controls. Additionally, both compounds are found in increased concentrations indoors compared to outdoors (2-fold for 2-methylpentane and 3-fold for 3-methylhexane) [62], suggesting that patients are exposed to higher concentrations than controls as they would spend more time indoors due to sarcoidosis-associated fatigue [13]. It is therefore unclear why these branched alkanes are exhaled in lower concentrations by patients compared to controls.

\subsubsection{Iodomethylcyclopentane and dibenzofuran}

Dibenzofuran can be found in tobacco smoke, oils, and other environmental sources, $[63,64]$ and there is no natural or environmental source of iodomethylcyclopentane. Interestingly, iodomethylcyclopentane is the only discriminatory VOC that was exhaled in a higher concentration in the patients compared to the controls. Since neither compound was found previously in exhaled breath [40], their likely source cannot be determined at this time.

\subsubsection{Conclusion}

This was the first study using an external validation in another hospital and at a different time point. A list of nine discriminatory VOCs was found in the discovery 
study. The initial results of this study are very promising but, as the results of our validation set already indicated, may not be reproducible in other studies. In order to achieve a reliable diagnostic breath fingerprint for sarcoidosis we encourage other groups/researchers to validate the presented findings.

Adding an external validation dataset is highly recommended in future clinical studies to ensure reproducibility so exhaled breath analysis can reliably be used in a clinical setting in the future. 


\section{Bibliography}

[1] Kyle Strimbu and Jorge A Tavel. What are biomarkers? Current opinion in HIV and AIDS, $5(6): 463-6$, nov 2010.

[2] Laura Caberlotto and Mario Lauria. Systems biology meets -omic technologies: novel approaches to biomarker discovery and companion diagnostic development. Expert Review of Molecular Diagnostics, 15(2):255-265, feb 2015.

[3] Gary S. Collins, Johannes B. Reitsma, Douglas G. Altman, and Karel G.M. Moons. Transparent Reporting of a multivariable prediction model for Individual Prognosis Or Diagnosis (TRIPOD): The TRIPOD Statement. Annals of Internal Medicine, 162(1):55, jan 2015.

[4] D. F. Ransohoff. Lessons from Controversy: Ovarian Cancer Screening and Serum Proteomics. JNCI Journal of the National Cancer Institute, 97(4):315-319, feb 2005.

[5] Eliot Marshall. Getting the noise out of gene arrays. Science (New York, N.Y.), 306(5696):6301 , oct 2004 .

[6] Anders Christiansen, Jesper Rømhild Davidsen, Ingrid Titlestad, Jørgen Vestbo, and Jan Baumbach. A systematic review of breath analysis and detection of volatile organic compounds in COPD. Journal of Breath Research, 10(3):034002, aug 2016.

[7] J. Cavaleiro Rufo, J. Madureira, E. Oliveira Fernandes, and A. Moreira. Volatile organic compounds in asthma diagnosis: a systematic review and meta-analysis. Allergy, 71(2):175-188, feb 2016 .

[8] Joachim D Pleil, Matthew A Stiegel, and Terence H Risby. Clinical breath analysis: discriminating between human endogenous compounds and exogenous (environmental) chemical confounders. Journal of Breath Research, 7(1):017107, mar 2013.

[9] Meggie Hakim, Yoav Y. Broza, Orna Barash, et al. Volatile organic compounds of lung cancer and possible biochemical pathways. Chemical Reviews, 112(11):5949-5966, nov 2012.

[10] C M Kneepkens, Guy Lepage, Claude C. Roy, et al. The potential of the hydrocarbon breath test as a measure of lipid peroxidation. Free Radical Biology and Medicine, 17(2):127-60, aug 1994.

[11] Wolfram Miekisch, Jochen K Schubert, and Gabriele F.E Noeldge-Schomburg. Diagnostic potential of breath analysisfocus on volatile organic compounds. Clinica Chimica Acta, 347(1):25-39, sep 2004.

[12] Gary W. Hunninghake, Ulrich Costabel, Masayuki Ando, et al. Statement on sarcoidosis. American Journal of Respiratory and Critical Care Medicine, 160(2):736-755, aug 1999.

[13] Marjolein Drent, Elyse E. Lower, and Jolanda De Vries. Sarcoidosis-associated fatigue. European Respiratory Journal, 40(1):255-263, jul 2012. 
[14] Marc A. Judson. The Clinical Features of Sarcoidosis: A Comprehensive Review. Clinical Reviews in Allergy and Immunology, 49(1):63-78, aug 2015.

[15] Robert P. Baughman, Daniel A. Culver, and Marc A. Judson. A concise review of pulmonary sarcoidosis. American Journal of Respiratory and Critical Care Medicine, 183(5):573-581, mar 2011.

[16] Elizabeth V Arkema, Johan Grunewald, Susanna Kullberg, et al. Sarcoidosis incidence and prevalence: a nationwide register-based assessment in Sweden. The European respiratory journal, 7(2):113-118, jul 2016.

[17] Shiv Saidha, Elias S. Sotirchos, and Christopher Eckstein. Etiology of sarcoidosis: Does infection play a role? Yale Journal of Biology and Medicine, 85(1):133-141, mar 2012.

[18] Edward S. Chen and David R. Moller. Etiologies of Sarcoidosis. Clinical Reviews in Allergy and Immunology, 49(1):6-18, aug 2015.

[19] Karen C. Patterson and Mary E. Strek. Pulmonary Fibrosis in Sarcoidosis. Clinical Features and Outcomes. Annals of the American Thoracic Society, 10(4):362-370, aug 2013.

[20] U Costabel and GW Hunninghake. ATS/ERS/WASOG statement on sarcoidosis. Sarcoidosis Statement Committee. American Thoracic Society. European Respiratory Society. World Association for Sarcoidosis and Other Granulomatous Disorders. European Respiratory Journal, 14(4), 1999.

[21] A Smolinska, A-Ch C Hauschild, R R R Fijten, et al. Current breathomicsa review on data pre-processing techniques and machine learning in metabolomics breath analysis. Journal of breath research, 8(2):27105, jun 2014.

[22] G. E. P. Box and D. R. Cox. An analysis of transformations. Journal of the Royal Statistical Society. Series B (Methodological, pages 211-252, 1964.

[23] B. Walczak and D.L. Massart. Wavelets in Chemistry, volume 22. Elsevier Science B.V, nov 2000 .

[24] Paul H. C. Eilers. A perfect smoother. Analytical Chemistry, 75(14):3631, jul 2003.

[25] Frank Dieterle, Alfred Ross, Götz Schlotterbeck, et al. Probabilistic quotient normalization as robust method to account for dilution of complex biological mixtures. Application in1H NMR metabonomics. Analytical Chemistry, 78(13):4281-4290, jul 2006.

[26] Foster Provost. Machine learning from imbalanced data sets 101. In Proceedings of the AAAI'2000 Workshop on ..., page 3, 2000.

[27] Haibo He, Yang Bai, Edwardo A. Garcia, and Shutao Li. ADASYN: Adaptive synthetic sampling approach for imbalanced learning. In Proceedings of the International Joint Conference on Neural Networks, pages 1322-1328. IEEE, jun 2008.

[28] Thanh N. Tran, Nelson Lee Afanador, Lutgarde M C Buydens, and Lionel Blanchet. Interpretation of variable importance in Partial Least Squares with Significance Multivariate Correlation (sMC). Chemometrics and Intelligent Laboratory Systems, 138:153-160, 2014.

[29] Yosef Hochberg and Yoav Benjaminit. Controlling the False Discovery Rate: A Practical and Powerful Approach to Multiple Controlling the False Discovery Rate: a Practical and Powerful Approach to Multiple Testing. Source Journal of the Royal Statistical Society. Series $B$ (Methodological) Journal of the Royal Statistical Society. Series B J. R. Statist. Soc. B, 57(1):289-300, 1995.

[30] Martin Rusilowicz, Michael Dickinson, Adrian Charlton, Simon O'Keefe, and Julie Wilson. A batch correction method for liquid chromatography???mass spectrometry data that does not depend on quality control samples. Metabolomics, 12(3):1-11, mar 2016.

[31] Agnieszka Smolinska, Ester M. M. EM Klaassen, JW Jan W. Dallinga, et al. Profiling of volatile organic compounds in exhaled breath as a strategy to find early predictive signatures of asthma in children. PLoS ONE, 9(4):e95668, apr 2014.

[32] John P. A. Ioannidis and Muin J. Khoury. Improving Validation Practices in Omics Research. Science, 334(6060), 2011. 
[33] Marcus R. Munafò, Brian A. Nosek, Dorothy V. M. Bishop, et al. A manifesto for reproducible science. Nature Human Behaviour, 1(1):0021, jan 2017.

[34] Michael Phillips, Thomas L. Bauer, Renee N. Cataneo, et al. Blinded Validation of Breath Biomarkers of Lung Cancer, a Potential Ancillary to Chest CT Screening. PLOS ONE, 10(12):e0142484, dec 2015.

[35] Paola Gramatica. Principles of QSAR models validation: internal and external. QSAR \&6 Combinatorial Science, 26(5):694-701, may 2007.

[36] Trevor Hastie, Robert Tibshirani, and J. H. (Jerome H.) Friedman. The elements of statistical learning : data mining, inference, and prediction.

[37] I. R. König, J. D. Malley, C. Weimar, et al. Practical experiences on the necessity of external validation. Statistics in Medicine, 26(30):5499-5511, dec 2007.

[38] Jan Hendrik Leopold, Lieuwe D J Bos, Peter J Sterk, et al. Comparison of classification methods in breath analysis by electronic nose. Journal of Breath Research, 9(4):046002, dec 2015.

[39] Stephen M. Rappaport, Sungkyoon Kim, Qing Lan, et al. Evidence that humans metabolize benzene via two pathways. Environmental Health Perspectives, 117(6):946-952, jun 2009.

[40] B de Lacy Costello, A Amann, H Al-Kateb, et al. A review of the volatiles from the healthy human body. Journal of breath research, 8(1):014001, mar 2014.

[41] J. King, P. Mochalski, K. Unterkofler, et al. Breath isoprene: Muscle dystrophy patients support the concept of a pool of isoprene in the periphery of the human body. Biochemical and Biophysical Research Communications, 423(3):526-530, 2012.

[42] Julian King, a Kupferthaler, K Unterkofler, et al. Isoprene and acetone concentration profiles during exercise on an ergometer. Journal of breath research, 3(2):027006, jun 2009.

[43] Kim DG G van de Kant, Linda JTM T M van der Sande, Quirijn Jöbsis, Onno CP P van Schayck, and Edward Dompeling. Clinical use of exhaled volatile organic compounds in pulmonary diseases: a systematic review. Respiratory research, 13(1):117, dec 2012.

[44] M A Spruit, M J Thomeer, R Gosselink, et al. Skeletal muscle weakness in patients with sarcoidosis and its relationship with exercise intolerance and reduced health status. Thorax, 60(1):32-38, jan 2005.

[45] A Korpi, J Jarnberg, and A L Pasanen. Microbial volatile organic compounds. Crit Rev Toxicol, 39(2):139-193, jan 2009.

[46] Yvonne J. Huang. Asthma microbiome studies and the potential for new therapeutic strategies. Current Allergy and Asthma Reports, 13(5):453-461, oct 2013.

[47] Etienne Riviere, Didier Neau, Xavier Roux, et al. Pulmonary streptomyces infection in patient with sarcoidosis, France, 2012. Emerging Infectious Diseases, 18(11):1907-1909, nov 2012.

[48] Adriana G. Guimarães, Jullyana S S Quintans, and Lucindo J. Quintans-Júnior. Monoterpenes with analgesic activity - A systematic review. Phytotherapy Research, 27(1):1-15, jan 2013.

[49] W A Duetz, H Bouwmeester, J B Van Beilen, and · B Witholt. MINIREVIEW Biotransformation of limonene by bacteria, fungi, yeasts, and plants. Appl Microbiol Biotechnol, 61(4):269277 , may 2003.

[50] L. A. Wallace. Major sources of benzene exposure. Environmental Health Perspectives, 82:165169 , jul 1989.

[51] Roy Harrison, Juana M. Delgado Saborit, Frédéric Dor, and Rogene Henderson. Benzene. 2010.

[52] P Schneider, I Gebefügi, K Richter, et al. Indoor and outdoor BTX levels in German cities. The Science of the total environment, 267(1-3):41-51, feb 2001.

[53] R.D Edwards and M.J Jantunen. Benzene exposure in Helsinki, Finland. Atmospheric Environment, 35(8):1411-1420, jan 2001.

[54] Jonathan Beauchamp, Jens Herbig, Rene Gutmann, and Armin Hansel. On the use of Tedlar@ bags for breath-gas sampling and analysis. Journal of Breath Research, 2(4):046001, dec 2008. 
[55] J.J.B.N. Van Berkel, J.W. Dallinga, G.M. Möller, et al. Development of accurate classification method based on the analysis of volatile organic compounds from human exhaled air. Journal of Chromatography B, 861(B, 861):101-107, jan 2008.

[56] Dillys van Vliet, Agnieszka Smolinska, Quirijn Jöbsis, et al. Can exhaled volatile organic compounds predict asthma exacerbations in children? Journal of Breath Research, 11(1):016016, mar 2017.

[57] James J. Schauer, Michael J. Kleeman, Glen R. Cass, and Bernd R T Simoneit. Measurement of emissions from air pollution sources. 2. C1 through C30 organic compounds from medium duty diesel trucks. Environmental Science and Technology, 33(10):1578-1587, may 1999.

[58] J. M. Daisey, A. T. Hodgson, W. J. Fisk, M. J. Mendell, and J. Ten Brinke. Volatile organic compounds in twelve California office buildings: Classes, concentrations and sources. Atmospheric Environment, 28(22):3557-3562, dec 1994.

[59] M. Phillips, R. N. Cataneo, J. Greenberg, et al. Effect of oxygen on breath markers of oxidative stress. European Respiratory Journal, 21(1):48-51, jan 2003.

[60] Diana Poli, Paolo Carbognani, Massimo Corradi, et al. Exhaled volatile organic compounds in patients with non-small cell lung cancer: cross sectional and nested short-term follow-up study. Respir Res, 6(1):71, dec 2005.

[61] Sahajal Dhooria and Dheeraj Gupta. Oxidative Stress in Sarcoidosis. pages 191-201. Springer New York, 2014.

[62] Barbara J. Finlayson-Pitts and James N. Pitts. Chemistry of the upper and lower atmosphere : theory, experiments, and applications. Academic Press, 2000.

[63] Meijun Li and Geoffrey S. Ellis. Qualitative and Quantitative Analysis of Dibenzofuran, Alkyldibenzofurans, and Benzo[ $<\mathrm{i}>\mathrm{b}</ \mathrm{i}>$ ]naphthofurans in Crude Oils and Source Rock Extracts. Energy \& Fuels, 29(3):1421-1430, mar 2015.

[64] M. Cooke. The Chemical Components of Tobacco and Tobacco Smoke, volume 71. CRC press, 2010. 
CHAPTER 6

Identification of microorganisms based on headspace analysis of volatile organic compounds by gas chromatographymass spectrometry

A Boots, A Smolinska, J van Berkel, $R$ Fijten, E Stobberingh, $M$ Boumans, $E$ Moonen, E Wouters, J Dallinga, F van Schooten J Breath Res. 2014 Jun 1;8(2):027106 


\subsection{Abstract}

The identification of specific volatile organic compounds (VOCs) produced by microorganisms may assist in developing a fast and accurate methodology for the determination of pulmonary bacterial infections in exhaled air. As a first step, pulmonary bacteria were cultured and their headspace analyzed for the total amount of excreted VOCs to select those compounds which are exclusively associated with specific microorganisms. Development of a rapid, noninvasive methodology for identification of bacterial species may improve diagnostics and antibiotic therapy, ultimately leading to controlling the antibiotic resistance problem.

Two hundred bacterial headspace samples from four different microorganisms (Escherichia coli, Pseudomonas aeruginosa, Staphylococcus aureus and Klebsiella pneumoniae) were analyzed by gas chromatographymass spectrometry to detect a wide array of VOCs. Statistical analysis of these volatiles enabled the characterization of specific VOC profiles indicative for each microorganism. Differences in VOC abundance between the bacterial types were determined using ANalysis of VAriance-principal component analysis (ANOVA-PCA). These differences were visualized with PCA. Cross validation was applied to validate the results.

We identified a large number of different compounds in the various headspaces, thus demonstrating a highly significant difference in VOC occurrence of bacterial cultures compared to the medium and between the cultures themselves. Additionally, a separation between a methicillin-resistant and a methicillin-sensitive isolate of S. aureus could be made due to significant differences between compounds. ANOVA-PCA analysis showed that 25 VOCs were differently profiled across the various microorganisms, whereas a PCA score plot enabled the visualization of these clear differences between the bacterial types.

We demonstrated that identification of the studied microorganisms, including an antibiotic susceptible and resistant S. aureus substrain, is possible based on a selected number of compounds measured in the headspace of these cultures. These in vitro results may translate into a breath analysis approach that has the potential to be used as a diagnostic tool in medical microbiology.

\subsection{Introduction}

The occurrence of detrimental respiratory tract infections embodies a great burden not only for patients and their health but also for the economy [1]. One of the most significant respiratory infections is pneumonia, a major health care problem affecting millions of individuals worldwide each year. Frequently underdiagnosed and undertreated pneumonia can promote development of multi-drug resistant bacteria. Respiratory tract infections are prevalent diseases in individuals older than 65 years of age, patients with impaired immune systems and chronically ill patients.

These infections, whether community- or hospital-acquired pneumonia (CAP versus HAP), can be life-threatening especially since the selection of antibiotic resistant pathogens is a growing concern due to the obvious necessity of antibiotic treatment $[2,3]$. Together, this translates into significant health care costs which are 
expected to grow with an ever ageing population. Particularly, the HAP ventilatorassociated pneumonia (VAP) is associated with high morbidity and mortality as well as with prolonged mechanical ventilation and length of stay in the intensive care unit (ICU) $[3,4]$.

On average, $20 \%$ of all patients receiving ventilation longer than $48 \mathrm{~h}$ will develop VAP, which makes this disease the most common nosocomial infection in ICU patients [5-8]. Other important respiratory microbial infections are those implicated in the sudden worsening of symptoms, also referred to as acute exacerbations, in various lung diseases including chronic obstructive pulmonary disease (COPD) and cystic fibrosis $(\mathrm{CF})$. These exacerbations are associated with increased morbidity and mortality and will thus lead to a decreased quality of life $[1,3,9,10]$. Although the exact cause of acute exacerbations is still unknown, risk factors include age, gender, lung function and a history of previous exacerbations $[1,10]$. Moreover, they are associated with pulmonary and systemic inflammations due to a change of the pulmonary microbial imbalance $[11,12]$. In general, the treatment of serious microbial infections demands immediate and specific medication, but often consists of broad-spectrum antibiotics mainly because the exact pathogenic cause is unknown.

Consequently, there is an urgent need for rapid diagnostic tests that can identify the pathogen(s) present. The development of such a test is of special importance since current diagnostics, aside from clinical criteria such as lung function, include sputum induction or bronchoscopy accompanied by sampling broncho-alveolar lavage fluid for quantitative laboratory microbiological analysis [13-15].

Although such an analysis can indeed more accurately detect the microbial cause, its usefulness is hampered by its invasiveness but mostly by the long duration of more than $48 \mathrm{~h}$ before results become available. Therefore, the development of rapid diagnostic tests to directly confirm the presence of a pathogen and to start accurate antimicrobial therapy is mandatory. Moreover, such a swift test can potentially improve clinical outcome allowing the selection of appropriate antimicrobial treatment for specific pathogens or by avoiding useless antibacterial treatment in the case of viral infection.

Quantitative and qualitative analysis of volatile organic compounds (VOCs) secreted by microbes responsible for pulmonary infections might provide a basis for early microbial identification in exhaled air. Analysis of exhaled air has the potential to revolutionize the diagnosis and treatment of bacterial infections since they are accompanied by a distinct smell which corresponds to certain VOCs [16]. VOCs are mainly released by microorganisms as metabolic products during growth, but also as secondary metabolites for protection against antagonists and competitors, or as signaling molecules for intercellular communication. Recent studies revealed the usefulness of single VOC analysis in evaluating bacterial growth in vitro [17-19]. However, a single VOC is not necessarily produced by one bacterium, thus hindering the ability to distinguish between specific species or strains. Therefore, well-defined combinations of 520 VOCs may provide higher discriminative power yielding volatile profiles with superior accuracies.

Exhaled air encompasses thousands of compounds (the volatome) and we have developed sophisticated computer data analysis tools for pipeline multivariate analysis in order to select discriminating VOC profiles of human breath. Using this approach, 
we have already successfully identified VOC-based biomarker profiles for the monitoring of inflammatory diseases of the intestines, liver and lungs [20-24].

The objective of this study was to analyze the volatome excreted by various microbial cultures using gas chromatography time-of-flight mass spectrometry (GC-tof-MS) and to determine which VOC profiles might enable rapid and easy identification of these cultures. We present a sampling and analysis methodology that is capable of identifying significantly different VOCs between diverse microbial cultures. Additionally, we report the successful use of ANalysis of VAriance-principal component analysis (ANOVA-PCA) to examine, and PCA to visualize, the classification of specific cultures with a high degree of accuracy.

\subsection{Materials and Methods}

\subsubsection{Samples}

Four different bacterial species were selected for this study: Escherichia coli (E. coli, ATCC 25922 and 35218), Pseudomonas aeruginosa (P. aeruginosa, ATCC 27853 and a clinical isolate), Staphylococcus aureus (S. aureus, clinical isolate) and Klebsiella pneumoniae (K. pneumonia, ATCC 700683). For S. aureus, two different strains, methicillin-resistant $S$. aureus (MRSA, clinical isolate) and methicillin-sensitive $S$. aureus (MSSA, clinical isolate), were also included. The methicillin resistance of both isolates was tested phenotypically and genotypically. The phenotype testing included determination of the minimum inhibitory concentration of methicillin. The genotype was tested by analyzing the presence of mec A (the gene responsible for methicillin resistance) as well as by performing multi-locus sequence typing and $S$. aureus protein A typing (Spa typing) to determine whether the two substrains were related.

For each microorganism, as well as for the two substrains of $S$. aureus, the headspace air of 40 cultures in total was collected and analyzed. For the microorganisms of which two sources were used (i.e. E. coli, P. aeruginosa and S. aureus), 20 replicates of each source were performed.

In order to achieve the highest degree of methodological standardization, 40 flasks containing only medium underwent the same procedure as the control samples. At the time of VOC sampling, the average CFU/ml was $109 / \mathrm{ml}$ for each genus.

\subsubsection{Sample collection and analysis}

Bacteria (obtained from ATCC) were grown on blood agar plates and incubated overnight at $37^{\circ} \mathrm{C}$. Next, bacteria were transferred into $4.5 \mathrm{ml}$ sterile Brain Heart Infusion broth (CM0225 Oxoid) and grown for $4 \mathrm{~h}$ with constant agitation at $37^{\circ} \mathrm{C}$. Subsequently, $0.5 \mathrm{ml}$ of the culture was transferred into $100 \mathrm{ml}$ sterile MuellerHinton broth (CM405 Oxoid) in 1 litre culture flasks. After overnight incubation at $37^{\circ} \mathrm{C}$, the culture flasks were flushed for 15 min with a total of $3000 \mathrm{ml}$ high-grade nitrogen connected to the inlet of a custom-made flask cap, designed to transport the contents of the headspace of the cultures under standardized conditions onto stainless steel two-bed sorption tubes connected to the outlet of the flask cap. The custom-made 
flask cap was designed to minimize air contamination on opening and connecting the flasks.

The used desorption tubes were packed with carbograph 1TD/Carbopack X (Markes International, Llantrisant, Wales, UK) that trap VOCs. For the analyses, the desorption tubes were placed inside a thermal desorption unit (Marks Unity desorption unit, Markes International) and subsequently heated to $270{ }^{\circ} \mathrm{C}$ in order to release all VOCs onto the gas chromatography capillary column (RTX-5ms, $30 \mathrm{~m}$ x $0.25 \mathrm{~mm}$ $5 \%$ diphenyl, 95\% dimethylsiloxane capillary, film thickness $1.0 \mu \mathrm{m}$ ). The desorption unit is highly suitable for repeated, quantitative and reproducible measurements.

VOCs were separated by GC (ThermoFisher Scientific, Austin, TX, USA) and subsequently detected by a time-of-flight mass spectrometer (tof-MS) (Thermo Electron Tempus Plus time-of-flight mass spectrometer, ThermoFisher Scientific, Austin, TX, USA). The temperature of the gas chromatograph was programmed as follows: $40^{\circ} \mathrm{C}$ for $5 \mathrm{~min}$, then raised by $10^{\circ} \mathrm{C} \mathrm{min}{ }^{-1}$ up to the final temperature of $270{ }^{\circ} \mathrm{C}$. This temperature was maintained for $5 \mathrm{~min}$. Electron ionization at $70 \mathrm{eV}$ was used combined with a $5 \mathrm{~Hz}$ scanning rate over a mass range of m/z $35350 \mathrm{amu}$.

An example of a bacterial headspace gas chromatogram is shown in Figure 6.1. Each peak represents a compound and the area underneath the peak is related to the occurrence of the compound concerned.

Now it has a line above figure saying where it was downloaded from.

\subsubsection{Data pre-processing}

Before multivariate analysis, pre-processing of the raw GC-tof-MS was performed. First, the start and end of each chromatogram (retention time $<1.3$ or $>23$ min respectively) were removed because of their noisy nature and the possible influence of column bleeding. The next data pre-processing step consisted of noise removal and baseline correction. De-noising was performed by means of wavelets [25], while the baseline was corrected using P-splines [26]. The shift in the peaks was eliminated by piecewise alignment method correlation optimized warping [27]. Subsequently, the chromatograms of each sample were merged by combining the corresponding compounds based on retention time and similarities in mass spectra (based on correlation).

To make all chromatograms comparable, the final step of pre-processing involved probabilistic quotient normalization after which the data were scaled using multivariate L1-median. In the headspace of the 240 samples, a total of 2465 compounds were detected by GC-tof-MS.

Since not all volatiles were present in all samples, the obtained data set contained a lot of zeros that did not provide important information regarding the origin of the samples. Consequently, in order to obtain a data set that is focused on only those compounds that convey information, the so-called $20 \%$ rule was applied [28]. This applied cutoff point was established in our previous studies and is currently used as the standard procedure. In short, this rule states that only variables that are nonzero for at least $20 \%$ of all samples in at least one of the experimental groups are used. After applying this rule, our data set consisted of 723 VOCs that were analyzed using multivariate analysis. 

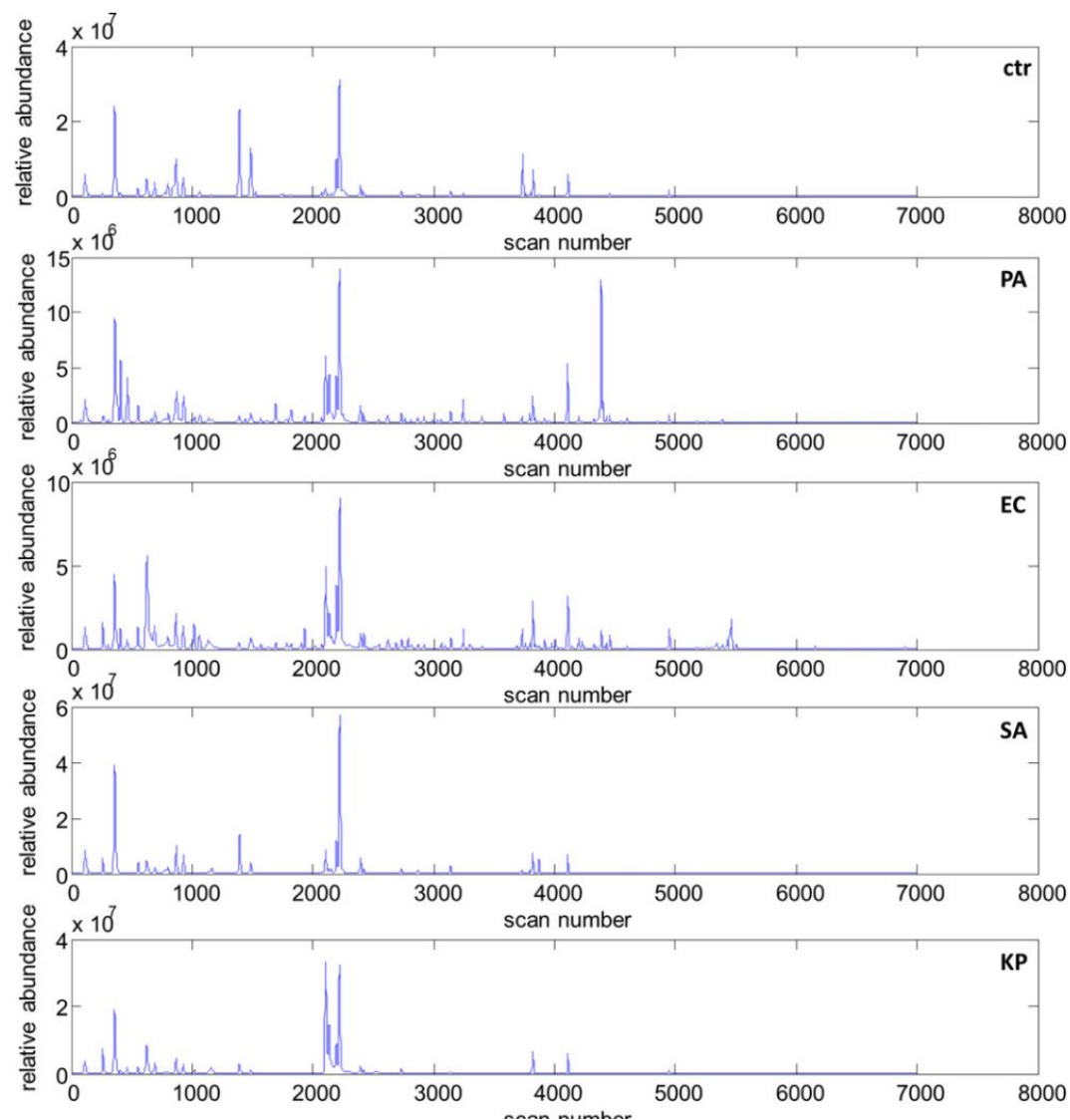

Figure 6.1: Average chromatograms obtained with GC-tof-MS for the control setup as well as for the four bacterial strains. $\mathrm{ctr}=$ medium only; $\mathrm{PA}=P$. aeruginosa; $\mathrm{EC}$ $=$ E. coli $; \mathrm{SA}=S$. aureus $; \mathrm{KP}=K$. pneumoniae.

Increasing the cutoff point decreased the number of VOCs in the final dataset, but this did not change the outcome of our analysis as the selected (significant) compounds were all present in at least $85 \%$ of the samples.

\subsubsection{Multivariate data analysis}

Multivariate analysis was used to extract relevant information from the numerically complex GC-tof-MS data. Since these data contain thousands of irrelevant and/or redundant compounds and the existence of a co-linearity between measured compounds, we performed variable (compound) selection that allowed picking the subset of best predictors. Compounds differently excreted across the various bacteria were selected based on a robust version of ANOVA-PCA [29, 30]. This supervised method is a combination of ANOVA and PCA.

ANOVA divides the overall variance in the data into the variance of the effect of 
interest (i.e. different bacteria groups) and of other irrelevant sources (e.g. medium effect). In this ANOVA part, the total variance is split into main effects and interaction effect. In this study, the main factors are the VOCs effect $(V)$ and the different bacteria plus medium $(B)$. In the ANOVA model, the measured head space data Xijk are assumed to be a result of the added effects of factors VOCs $(V)$ and the bacteria $(B)$ over VOC $i$, bacteria $j$ and culture $k$. For head space data, the ANOVA model is given in equation 6.1 and is assumed to be the additive result of the general mean $(\mu)$, main effects and their interactions:

$$
X_{i j k}=\mu+V_{i}+B_{j}+V B_{i j}+e_{i j k}
$$

The interaction effect $\left(V B_{i j}\right)$ is of the most interest in this study as it reflects which VOCs are differentially profiled across various bacteria and medium when all othereffects are averaged out. Next, PCA is performed on the matrix of interaction effects $V B_{i k}$ [31] to identify VOCs that significantly change between various bacteria and medium.

If the bacterium $(j)$ consumes or produces the compound $(i)$, the interaction effect VBik is significantly different from zero and this indicates the intensity of the compound, i.e. its relative amount is affected.

In order to obtain statistically significant VOCs, the Hotelling T2 distribution (which corresponds to the multivariate t-test) was used. The compounds of which the concentration is affected by the microorganism present are expected to have a p-value smaller than or equal to 0.05 . In order to visualize the differences between the VOCs present in the headspace of the various bacteria, PCA analysis was applied on the headspace data containing only relevant VOCs. The summary of all bacteria-related VOCs and their relationship is represented in a PCA score plot, whereas the relative abundance of the relevant VOCs in the headspace of the various bacteria strains is depicted in a PCA bi-plot. A detailed description of the features of a PCA score plot and a bi-plot was given previously [28]. Since MSSA and MRSA are biologically closely related, we used partial least-square discriminant analysis with cross model validation (PLS-DA CMV) to find compounds differently profiled in the headspace of these two strains [32]. In this method, nested cross validation is included in the variable selection procedure, meaning that the classification model will be built on a training set and validated on a test set. This approach enables removing irrelevant compounds, thereby optimizing the PLS-DA model for accurate classification of MSSA and MRSA. In our study, we have used the CMV approach presented in Gidskehaug et al and all MATLAB routines for performing variable selection described in this study [33].

\subsection{Results}

The first step of the data analysis was creating average chromatograms for all four microorganisms and the medium control in order to visually examine whether clear differences could be observed in the pattern of VOCs. These average chromatograms are depicted in figure 6.1 and clearly show that each tested condition resulted in a distinct peak pattern, suggesting that different VOCs are present in the headspace air of the various bacteria. 
Next, the data matrix containing 240 samples and 723 VOCs was analyzed using ANOVA-PCA to select the significant compounds affected by the presence of the various microorganisms in comparison to the medium control and each other. This approach resulted in the selection of 25 significantly different VOCs with p-values $<0.05$. These VOCs were putatively chemically identified based on the NIST library and the analysis of the retention time and the mass spectrum by an experienced massspectrometrist Table 6.1. Their relationship toward the various microorganisms or control setting, and thus their contribution to an adequate identification of a sample, is also depicted in this table.

Table 6.1: The 20 chemically identified VOCs of the overall 25 discriminating volatiles used to correctly classify the four bacterial strains tested. The reported change in abundance is with respect to the remaining groups. ctr = medium only; $\mathrm{PA}=P$. aeruginosa $; \mathrm{EC}=E$. coli $; \mathrm{SA}=S$. aureus $; \mathrm{KP}=K$. pneumoniae.

\begin{tabular}{lll}
\hline Nr. & Name of compound & Abundance \\
\hline 1 & Acetone & $\uparrow \mathrm{SA}$ \\
2 & Isoprene & $\uparrow \mathrm{EC}, \mathrm{KP}$ and PA \\
3 & 1-propanol & $\uparrow \mathrm{EC}$ and KP \\
4 & 3-methylbutanal & $\downarrow \mathrm{EC}, \mathrm{KP}, \mathrm{PA}$ and SA \\
5 & 2-methylbutanal & $\downarrow \mathrm{EC}, \mathrm{KP}, \mathrm{PA}$ and SA \\
6 & 4-methylcyclohexene & $\uparrow \mathrm{KP}$ \\
7 & Dimethyl disulfide & $\uparrow \mathrm{SA}$ \\
8 & 2,3,3-trimethylpentane & $\downarrow \mathrm{EC}, \mathrm{KP}, \mathrm{PA}$ and SA \\
9 & Benzaldehyde & $\downarrow \mathrm{EC}, \mathrm{KP}, \mathrm{PA}$ and SA \\
10 & 1-undecene & $\uparrow \mathrm{PA}$ \\
11 & 2-pentene & $\uparrow \mathrm{PA}$ \\
12 & Acetic acid & $\uparrow \mathrm{EC}$ \\
13 & 2,3-butanedione & $\downarrow \mathrm{EC}, \mathrm{KP}, \mathrm{PA}$ and SA \\
14 & 2-butanone & $\uparrow \mathrm{PA}$ and KP \\
15 & 1,1,2,2-tetrachloroethane & $\uparrow \mathrm{SA}$ \\
16 & N-propylacetate & $\uparrow \mathrm{EC}$ \\
17 & 2-heptanone & $\uparrow \mathrm{PA}$ and EC \\
18 & Dimethyltrisulfide & $\uparrow \mathrm{SA}$ \\
19 & 2-nonanone & $\uparrow \mathrm{PA}$ \\
20 & Indole & $\uparrow \mathrm{EC}$ \\
21 & 3-methylheptane & $\uparrow \mathrm{EC}$ \\
22 & 1-methyl-4-(1-methylethenyl)cyclohexane & $\downarrow \mathrm{EC}, \mathrm{KP}, \mathrm{PA}$ and SA \\
23 & 3-methylcyclohexene & $\uparrow \mathrm{KP}$ \\
24 & Branched $\mathrm{C}_{14} \mathrm{H}_{30}$ & $\uparrow \mathrm{KP}$ \\
25 & Branched $\mathrm{C}_{14} \mathrm{H}_{30}$ & $\uparrow \mathrm{KP}$ \\
& &
\end{tabular}

Visualization of the observed discriminating VOCs was performed with a PCA score plot resulting from a separate PCA analysis using only these 25 selected compounds (Figure 6.2). This plot clearly shows that the medium control and the four different microorganisms could be well separated along three first principal compo- 
nents, explaining $26.5 \%$ of the total variance. The medium control group is well separated from the four different bacteria, although a few points seem to be outliers as they share some similarities with the $P$. aeruginosa samples. The headspace air samples of $S$. aureus and $K$. pneumoniae produce very dense and compact data clouds, revealing small variations within these two groups. The samples corresponding to the other two bacteria, i.e. E. coli and P. aeruginosa, are somewhat more spread, which is indicative of more variation.

Interestingly, the main variation in the data (accounting for $12 \%$ of the total variance) corresponds to a complete separation of $P$. aeruginosa and E. coli from $S$. aureus and $K$. pneumoniae. This separation along PC1 indicates that the VOCs secreted or absorbed by $P$. aeruginosa share similarities with those secreted or absorbed by $E$. coli but are different from those present in the headspace air of the other two bacteria tested. Similarly, S. aureus and K. pneumoniae display comparable characteristics in their present VOCs and are at the same time distinct from those present in the headspace of the other two microorganisms.

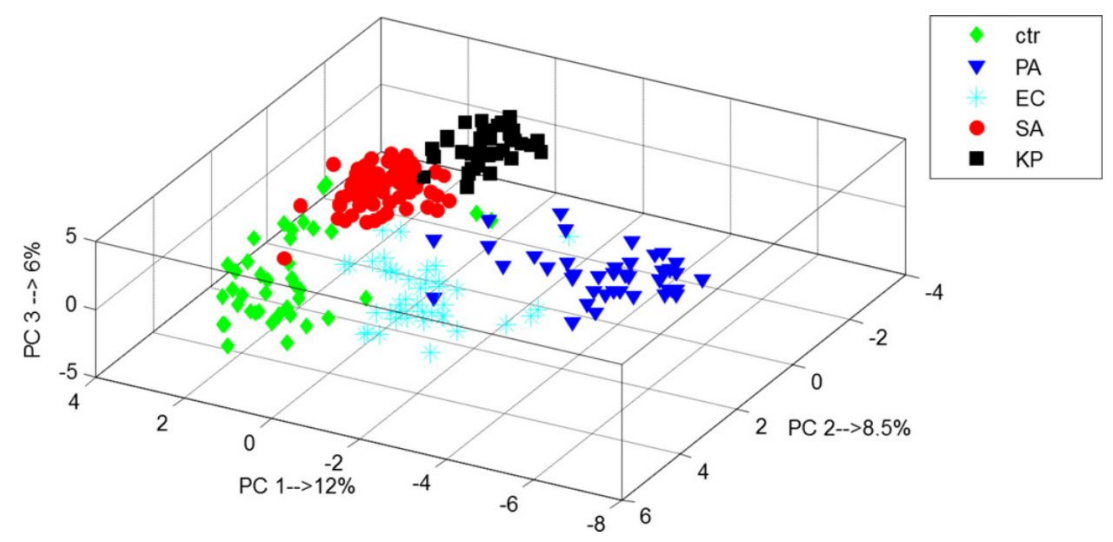

Figure 6.2: PCA score plot based on the 25 VOCs selected via ANOVA-PCA. As each point represents a single bacterium, this plot provides a summary of all bacteria and shows how they are related to each other. Hence, the points that are close to each other have a similar profile, while objects that lie far away from each other are characterized by different properties. ctr $=$ medium only; $\mathrm{PA}=P$. aeruginosa $; \mathrm{EC}$ $=$ E. coli $; \mathrm{SA}=S$. aureus $; \mathrm{KP}=K$. pneumoniae.

As can be seen in the average chromatograms of the $S$. aureus substrains, the MSSA and the MRSA, the VOC profiles present in these headspace air samples were quite similar (Figure 6.3). However, some very differently secreted or absorbed VOCs could also be found between the two sample sets.

PLS-DA CMV was used to find differentially profiled VOCs. The final PLS-DA model was built using only two latent variables. This simple classification model allowed correct prediction of $81 \%$ of the independent test samples of both $S$. aureus strains. In total, 87 VOCs were selected as discriminatory within the applied PLSDA CMV procedure. The eight most distinct and descriptive volatiles were visualized 
using a PCA bi-plot (Figure 6.4(a)).
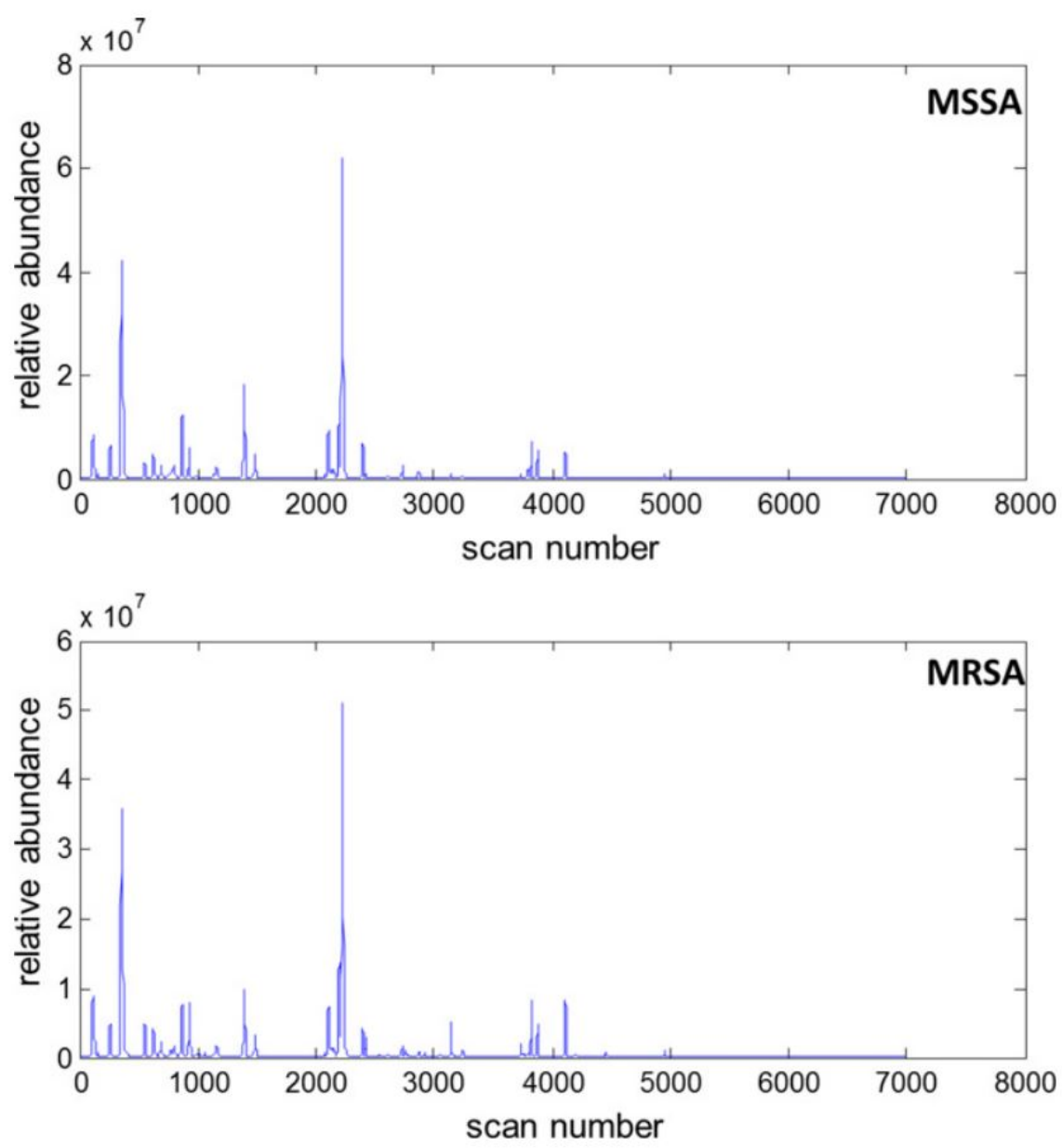

Figure 6.3: Average chromatograms obtained with GC-tof-MS for the methicillin -sensitive and methicillin-resistant strains of $S$. aureus. MSSA = methicillin -sensitive S. aureus; MRSA = methicillin-resistant $S$. aureus

Within this plot, the dashed lines indicate the changes in the relative concentrations of the corresponding compounds. As can be deducted from figure 6.4(a), the MSSA samples display far more variation than the MRSA samples although most of the variation can be explained by only a few outliers. Moreover, seven of the eight discriminating VOCs show increased levels in the MRSA samples, suggesting that they are specifically produced by this $S$. aureus substrain. The only volatile that is increased in the MSSA samples and of which the presence is thus positively indicative of this subclass is 1,1,2,2-tetrachloroethane (figure 6.4(b)). Additionally, the angle between these dashed lines in the bi-plot represents the similarity between the com- 


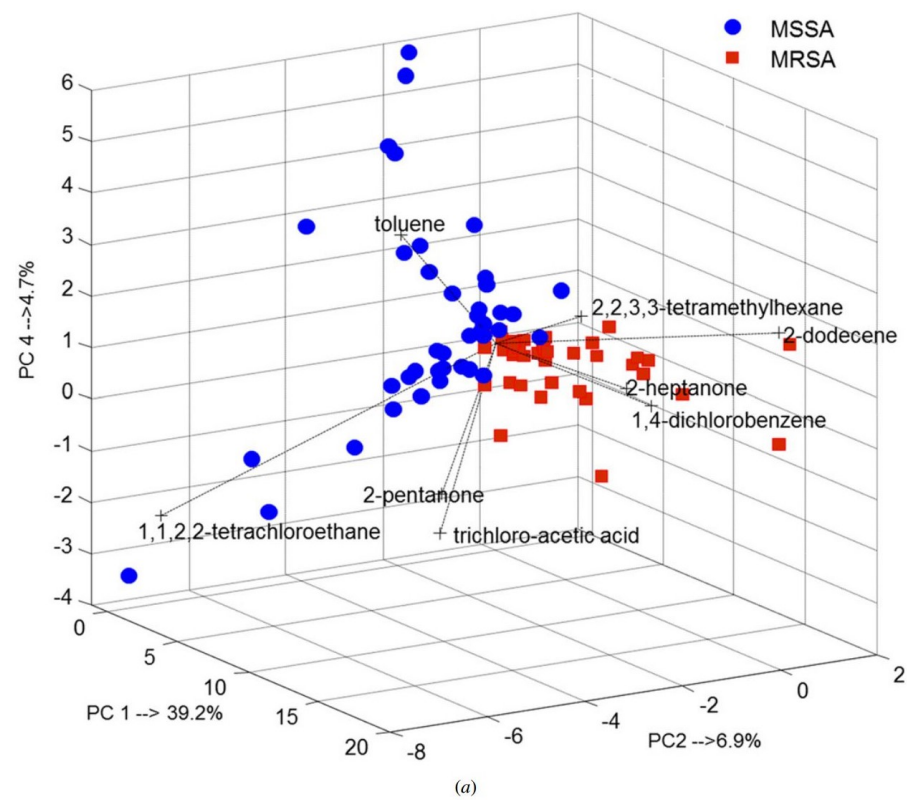

Figure 6.4: Panel (a) Bi-plot of PCA analysis performed on the significant VOCs excreted in the headspace air of MSSA (methicillin-sensitive S. aureus) and MRSA (methicillin-resistant $S$. aureus). Every point corresponds to a single measurement and the lines to the eight most important VOCs. The angle between compounds mirrors their correlation, i.e. a small angle means the compounds are positively correlated (i.e. their relative abundance changes the same way). Additionally, the abundance of a compound is elevated if the sign of a new coordinate (i.e. PCs) for samples and compound is the same (i.e. either both positive and both negative). Panels $(b)(d)$ Boxplots of three exemplary VOCs (i.e. 1,1,2,2-tetrachloroethane, 2heptanone and 1,4-dichlorobenzene) that are significantly different excreted in the headspace air of MSSA and MRSA.

pounds. In other words, the smaller the angle between two compounds, the larger the correlation between them. For instance, the angle between the volatiles 2-heptanone and 1,4-dichlorobenzene is very small, indicating that their presence will be influenced in the same way and thus show similar trends for both groups. Indeed, their levels are both elevated in MRSA and around zero in MSSA as is shown in figures $6.4(c)$ and $(d)$. The very large angle between these two compounds and for instance 1,1,2,2tetrachloroethane implies that their concentrations will be affected in opposite ways as can indeed also be deduced from their boxplots (figures $6.4(b)(d)$ ).

\subsection{Discussion}

All organisms generate general and specific VOCs as part of their normal metabolism and therefore sometimes can be distinguished based on a distinct smell in vivo as well 

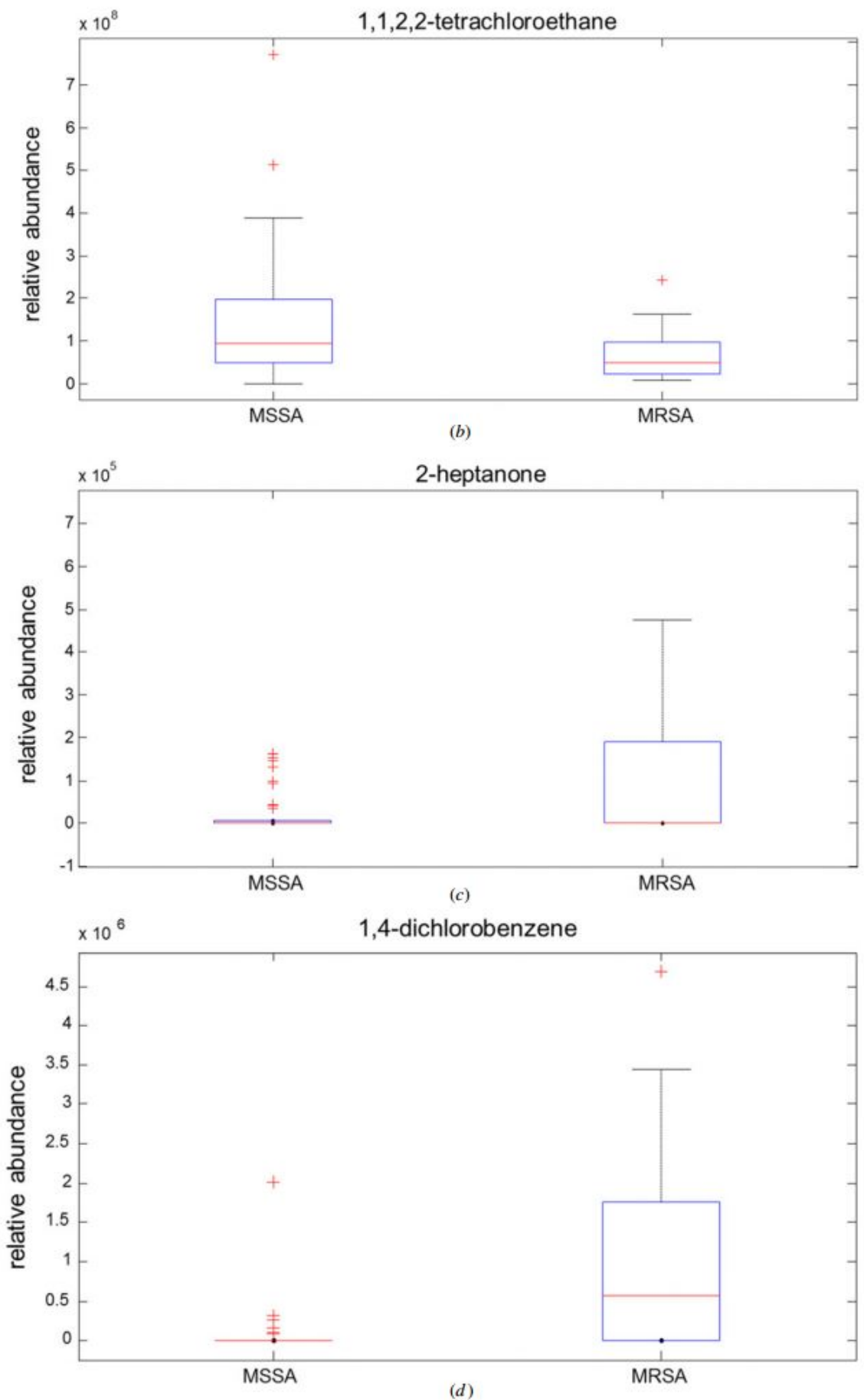

Figure 6.4: (Continued.) 
as in vitro [34-36]. For microorganisms, it is known that they produce a variety of VOCs due to their metabolic and oxidative actions.

A recent systemic overview of the distinct VOCs produced by a selection of pathogenic microorganisms [38] suggests that the majority of microbial volatiles can be divided into the following groups: (1) hydrocarbons produced via the methylerythritol phosphate pathway or $\beta$-oxidation of fatty acids, (2) alcohols produced through the oxidation of fatty acids, (3) acids due to anaerobic metabolism, (4) aldehydes derived from oxidative metabolism, (5) ketones produced during decarboxylation of fatty acid derivatives, (6) cyclic compounds of which the biological origin remains largely unknown, (7) esters as a result of esterification between acetic acid and alcohols, (8) S-containing compounds mostly of unknown origin and (9) N-containing compounds resulting from amino acid breakdown [37-39].

On an individual level, various VOCs are already identified to be specific for bacterial microorganisms and an overview of these volatiles has recently been reviewed by Bos et al [37]. It has been reported that $S$. aureus cultures are characterized by dimethyl sulfide, isovaleric acid, 2-methyl-2-butanal and ammonia $[17,37,40]$ and $P$. aeruginosa cultures by 1-undecene, 2,4-dimethyl-1-heptane, 2-butanone, 4-methylquinazoline, methyl thiocyanide, dodecane and hydrogen cyanide [37, 38, 40-43].

Additionally, increased production of indole [43, 44] and octan-1-ol, and ethanol [43] has been reported for E. coli, whereas $K$. pneumoniae cultures are known to produce 3-hydroxy-2-butanone and 1-hydroxy-2-propanone [45]. Interestingly, some volatiles are not specifically produced by one pathogen but are shared by different species of bacteria.

For instance, the volatiles 2-pentanone, formaldehyde, methyl mercaptan and trimethylamine are produced by all clinically relevant pathogens studied thus far but not by humans, and can serve as a biomarker for their presence [37]. Additionally, hexanal is not only produced by $S$. aureus but also by $S$. pneumoniae [46]. Another example is the volatile dimethylsulfide which is produced by almost all gram negative bacterial species including E. coli, $K$. pneumoniae and P. aeruginosa [37, 42, 43].

The overlapping production of VOCs by various bacteria suggests that a single VOC does not have enough discriminating power to separate specific strains of microorganisms. Therefore, we believe that VOC profiles consisting of a unique and distinct combination of volatiles are required to discriminate between microbes. Interestingly, the usefulness of VOC profiles in differentiating bacterial monocultures grown in vitro has recently been shown [44].

In this study, we used GC-tof-MS for the headspace analysis of cultures from four different bacterial species, $K$. pneumoniae, $P$. aeruginosa, $S$. aureus and $E$. coli, as well from a methicillin-resistant and -sensitive $S$. aureus (MRSA versus MMSA). The first three microorganisms were specifically chosen as they are among the most frequent reported causes of pulmonary infections and exacerbations occurring in various lung diseases including ventilator-associated pneumonia, COPD and CF $[6,8,10,12,47-49]$. Additionally, E. coli was chosen since this gram-negative bacterium is a known inducer of both HAP and VAP and is currently reported to be often as responsible for ICU infections as $P$. aeruginosa [50]. Indeed, it is clinically important, but until now also difficult, to quickly and correctly distinguish these two microorganisms from each other. As depicted in figure 6.2, the four se- 
lected microorganisms could be well distinguished from the medium control as well as from each other. This discrimination was based on 25 specific VOCs and their putative chemical identification is shown in table 6.1. Interestingly, seven of these discriminative volatiles appeared to be lower or even absent in the air above bacterial cultures compared with control headspace samples, indicating that these compounds are potentially metabolized or taken up by the bacteria.

The fact that the other discriminative volatiles were more abundant in the headspace of specific microbial air samples suggests that these VOCs are due to the formation of metabolic products by the bacteria. The overall observed differences in VOC changes further underline the finding of others that metabolic changes lead to the altered excretion of VOCs and that various individual volatiles can be assigned to certain bacterial species $[38,39,51]$.

Table 6.1 demonstrates that most discriminative VOCs belong to four of the classes of volatiles reported to be differentially produced by various bacteria, namely long chain hydrocarbons $(\mathrm{n}=7)$, ketones $(\mathrm{n}=5)$, cyclic compounds $(\mathrm{n}=3)$ and aldehydes $(\mathrm{n}=3)$.

Most discriminative VOCs are hydrocarbons, volatiles presumably derived from the oxidation of fatty acids, a process known as lipid peroxidation. These hydrocarbons include isoprene, 4-methylcyclohexene, 3-methylheptane, 2-pentene, 1-undecene and both $\mathrm{C} 14 \mathrm{H} 30$ isomers. This finding is in accordance with the literature, as it has already been reported that bacteria excrete volatiles associated with lipid peroxidation [16]. In contrast to the majority of observed hydrocarbons and to our expectations, the hydrocarbon 2,3,3-trimethylpentane is actually decreased in the headspace of all bacteria. Additional research is needed to confirm this finding and understand its full meaning. From the observed ketones, known to be produced during decarboxylation of fatty acids, 2,3-butanedione is surprisingly decreased in the headspace air of all bacteria. The ketones 2-butanone, 2-heptanone and 2-nonanone are increased in the headspace of $P$. aeruginosa cultures in accordance to previous findings $[39,42,52,53]$. The last VOC of this group, acetone, was present in elevated levels in the headspace air of $S$. aureus and this finding is also in accordance with the literature $[44,54]$. The selected cyclic compounds 4-methylcyclohexene and 3-methylcyclohexene are specifically and solely excreted by $K$. pneumoniae, indicating that these VOCs are very specific for this microorganism. To our knowledge, we are the first to couple these specific compounds to this bacterium. Interestingly, the other cyclic compound, 1-methyl-4-(1-methylethenyl)cyclohexane, was decreased in the headspace air of all bacteria, suggesting that this volatile was either absorbed or potentially metabolized by the microorganisms. Finally, the distinct aldehydes 2-methylbutanal, 3-methylbutanal and benzaldehyde are all significantly decreased in the headspace air of the four tested bacteria.

Although this finding appears striking due to the fact that the excretion of aldehydes is reported to be related to oxidative metabolism and lipid peroxidation [37], there are already some studies showing decreased levels of aldehydes including 2methylbutanal in the air excreted by E. coli, P. aeruginosa and $K$. pneumoniae $[17,44,55]$. The remaining seven discriminating VOCs are equally distributed over the other classes and more specific for individual bacteria. The alcohol propanol is present in increased amounts in samples of both E. coli and K. pneumoniae and thus 
already displays more discriminative power than those volatiles present in almost all bacterial headspace air samples.

Additionally, some volatiles reported in the literature to be unique for a microorganism are also observed to be significantly changed in the headspace air of our samples. For instance, the S-containing compounds dimethylsulfide and dimethyltrisulfide are distinctly increased in $S$. aureus headspace, whereas indole, reported to be a metabolic byproduct of $E$. coli, is present as the most significantly different compound in the $E$. coli samples [17, 37, 44]. Interestingly, also N-propylacetate and acetic acid are specifically present in $E$. coli samples as reported previously [56-58].

Finally, our study is the first to reveal that $S$. aureus cultures are also capable of producing chlorine-containing compounds of which 1,1,2,2-tetrachloro- ethane is the most distinct for this microorganism. With regard to the two S. aureus strains, the number of significantly different VOCs between them was lower than those demonstrating significant differences between the various microorganisms. This finding indicates that the VOC profiles of these two different strains of one microorganism display more similarity than those obtained from different microbial species.

Nevertheless, it is still possible to correctly distinguish the antibiotic- sensitive and -resistant strain based solely on their excreted VOC profiles. Among the eight most discriminating volatiles used to separate MSSA from MRSA, two chlorine-containing compounds are observed, namely trichloroacetic acid and 1,4-dichloroacetic acid. This finding is of special interest as the combined group of all $S$. aureus substrain cultures together has also been shown to produce a chlorine-containing compound as the most distinct volatile. Further research has to elucidate the origin and importance of these compounds in $S$. aureus cultures.

The micro-organisms described in this study were not grown in different types of (commercial) media even though it can be anticipated that the excreted VOC profile will be influenced hereby. However, we chose not to test different media in this stage of the research in order to achieve a high degree of standardization by employing a robust methodology that was not influenced by too many confounding factors. In our opinion, our chemical analysis, data handling and accurate data mining thus provide a highly reproducible methodology [23]. In the present study, this approach has resulted in a more homogeneous database of detected VOCs, thereby increasing the power of our statistical analysis to extract those VOCs that actually discriminate between different micro-organisms. Upon putatively identifying these specific VOCs, the next step of our research will include analyzing the effect of different confounders on the observed discriminating compounds. These factors include (1) different culture media, (2) the presence of more than one specific micro-organism and (3) the effect of different antibiotics. With respect to the first point, recent encouraging reports indicate that many VOCs emitted by $S$. aureus, E. coli and P. aeruginosa in various culture media are identical, suggesting that micro-organisms indeed emit such strainspecific and medium-independent VOCs when grown in rich culture media under anaerobic conditions [59-61].

The second point has briefly been addressed in a recent study by mixing $S$. aureus and $P$. aeruginosa in different proportions [60]. The results reported show that VOCs excreted by such a mixture not only allow the identification of the specific pathogens present but also reflect the proportion of each of them due to their ratios [60]. Finally, 
regarding the last point, it has recently been proven that co-incubating specific microorganisms with antibiotics indeed results in altered VOC production, confirming that the analysis of exhaled air might also be useful for the determination of antibiotic susceptibility [17, 62]. Our encouraging results regarding the separation between MSSA and MRSA samples further underline that VOC profiles might differ depending on the microbial sensitivity toward antibiotics.

Taken together, identifying unique combinations of discriminating VOCs under variable conditions is the next step forward toward designing and testing VOC profiles capable of identifying specific microorganisms in primary patient samples. As a first step in this in vitroin vivo translation, our group has recently shown that the presence of $P$. aeruginosa cultures in the sputum of CF patients can correctly be classified using 14 distinct VOCs [63]. Putative chemical identification of these discriminating volatiles is warranted in order to compare them with the observed in vitro VOC profile of $P$. aeruginosa to check whether bacterial VOCs measured under anaerobic culturing conditions indeed reflect the VOCs relevant in vivo in human diseases. However, the limited number of samples in this study would make such a comparison too premature.

Until now, only a few studies have tried to compare in vitro and in vivo breathomics data and these comparisons have not yet included putatively chemically identified VOCs. Instead, only specific volatiles such as 2-amino- acetophenone [64] and hydrogen cyanide [65] or total fingerprints (i.e. peak numbers) have been compared [66]. The fact that GC-MS does not enable real-time measurements could be regarded as a disadvantage of our methodology. However, it should be noted that if continuous on-line monitoring is not required, the disadvantage of not measuring in vivo is easily overcome by loading the samples onto desorption tubes that trap and stabilize VOCs for at least one year. Measuring a stored sample only takes roughly $30 \mathrm{~min}$, indicating that rapid analysis is still possible as long as the apparatus and required algorithms are available on site. To identify a bacterial infection at the ICU even more quickly, the continuous presence of a big, expensive real-time instrument as well as an experienced technician and statistician would be required on every ICU. Additionally, the disadvantage of not measuring real-time can even be turned into an advantage when applied for scientific studies instead of diagnostics. After all, off-line sampling and storage on desorption tubes implicates that samples from various locations, collected over a long time, can be measured by one instrument in a short time, thereby eliminating a good deal of instrumental variation.

In this study we sampled all cultures at the same time point (i.e. after the addition of the cultures to the medium) and only performed one measurement on each sample. An alternative to GC-MS would be the use of proton transfer reaction mass spectrometry (PTR-MS) that does support continuous measurements. However, this methodology is not able to detect compounds with low proton affinity, such as simple hydrocarbons, and is also not suited to identify compounds with a high degree of accuracy since PTR-MS cannot differentiate between compounds with the same molecular weight and does not provide identification by means of mass spectra [36, 62]. On top of that, fragmentation and clustering of product ions further complicate the qualitative interpretation of the mass spectra and require detailed correlation analyses of the observed signals in PTR-MS [56]. Since our aim was to map and identify the discriminating VOCs from headspace with a high degree of accuracy, GC-MS was the 
most suitable methodology.

In conclusion, this study demonstrates the ability to identify specific microorganisms based on only a limited amount of compounds measured in the headspace of these cultures. Such a selection and identification of interesting VOCs is the first step toward developing and optimizing diagnostic tools that specifically detect the presence of microorganisms directly in breath samples from patients in a fast, sensitive, non-invasive and cost-effective way. Until such dedicated sensor-based detectors are developed, however, the presented GC-MS methodology is already of great value since the analyses are fast and results can thus be obtained within a short time. Additionally, the present method is very cost-effective and demonstrates a high degree of sensitivity and specificity. 


\section{Bibliography}

[1] A. Anzueto. Impact of exacerbations on copd. European Respiratory Review, 19(116):113-118, jun 2010.

[2] Marc J M Bonten. Ventilator-associated pneumonia: Preventing the inevitable. Clinical Infectious Diseases, 52(1):115-121, jan 2011.

[3] Noyal Mariya Joseph, Sujatha Sistla, Tarun Kumar Dutta, Ashok Shankar Badhe, and Subhash Chandra Parija. Ventilator-associated pneumonia: A review. European Journal of Internal Medicine, 21(5):360-368, oct 2010.

[4] M W Pletz, O Burkhardt, and T Welte. Nosocomial methicillin-resistant Staphylococcus aureus (MRSA) pneumonia: linezolid or vancomycin? - Comparison of pharmacology and clinical efficacy. Eur.J Med.Res., 15(0949-2321 (Print)):507-513, nov 2010.

[5] D C Bergmans. Ventilator-associated pneumonia: studies on pathogenesis, diagnosis and prevention. Phd thesis, Maastricht University, 1999.

[6] Jean Chastre and Jean-Yves Fagon. Ventilator-associated Pneumonia. American Journal of Respiratory and Critical Care Medicine, 165(23):867-903, apr 2002.

[7] Madiha Ashraf and Luis Ostrosky-Zeichner. Ventilator-Associated Pneumonia: A Review. Hospital Practice, 40(1):93-105, jan 2012.

[8] David R Park. The Microbiology of Ventilator-Associated Pneumonia Features of Specific Common VAP Pathogens The Prevalence of Routine Bacterial Pathogens in VAP VAP in Patients After Tracheotomy VAP in Patients With Traumatic Injuries. Respiratory care, 50(6):742-765, jun 2005.

[9] Julia Emerson, Margaret Rosenfeld, Sharon McNamara, Bonnie Ramsey, and Ronald L. Gibson. Pseudomonas aeruginosa and other predictors of mortality and morbidity in young children with cystic fibrosis. Pediatric Pulmonology, 34(2):91-100, aug 2002.

[10] I S Patel, T A R Seemungal, M Wilks, et al. Relationship between bacterial colonisation and the frequency, character, and severity of COPD exacerbations. Thorax, 57(9):759-64, sep 2002.

[11] Ronald L. Gibson, Jane L. Burns, and Bonnie W. Ramsey. Pathophysiology and Management of Pulmonary Infections in Cystic Fibrosis. American Journal of Respiratory and Critical Care Medicine, 168(8):918-951, oct 2003.

[12] Sanjay Sethi and Timothy F Murphy. Infection in the pathogenesis and course of chronic obstructive pulmonary disease., nov 2008.

[13] Ben Nicholas and Ratko Djukanović. Induced sputum: a window to lung pathology. Biochemical Society transactions, 37(Pt 4):868-872, aug 2009. 
[14] Paula Ramírez, Mauricio Valencia, and Antoni Torres. Bronchoalveolar lavage to diagnose respiratory infections. Seminars in Respiratory and Critical Care Medicine, 28(5):525-533, oct 2007.

[15] Annemarie Sykes, Patrick Mallia, and Sebastian L Johnston. Diagnosis of pathogens in exacerbations of chronic obstructive pulmonary disease. Proceedings of the American Thoracic Society, 4(8):642-6, dec 2007.

[16] Stefan Schulz and Jeroen S Dickschat. Bacterial volatiles: the smell of small organisms. Natural product reports, 24(4):814-842, aug 2007.

[17] Randall A. Allardyce, Alex L. Hill, and David R. Murdoch. The rapid evaluation of bacterial growth and antibiotic susceptibility in blood cultures by selected ion flow tube mass spectrometry. Diagn. Microbiol. Infect. Dis., 55(4):255, aug 2006.

[18] Robin Michael Statham Thorn and John Greenman. Microbial volatile compounds in health and disease conditions. Journal of Breath Research, 6(2):024001, jun 2012.

[19] A. Critchley, T. S. Elliott, G. Harrison, et al. The proton transfer reaction mass spectrometer and its use in medical science: Applications to drug assays and the monitoring of bacteria. International Journal of Mass Spectrometry, 239(2-3):235-241, dec 2004.

[20] Agnes W Boots, Joep J B N van Berkel, Jan W Dallinga, et al. The versatile use of exhaled volatile organic compounds in human health and disease. Journal of Breath Research, $6(2): 27108$, jun 2012 .

[21] J. W. Dallinga, C. M H H T Robroeks, J. J B N Van Berkel, et al. Volatile organic compounds in exhaled breath as a diagnostic tool for asthma in children. Clinical and Experimental Allergy, 40(1):68-76, jan 2010.

[22] J J B N Van Berkel. There's something in the air - Volatile organic compounds in exhaled breath in pulmonary diseases. Phd thesis, Maastricht University, 2010.

[23] J. J B N Van Berkel, J. W. Dallinga, G. M. Möller, et al. A profile of volatile organic compounds in breath discriminates COPD patients from controls. Respiratory Medicine, 104(4):557-563, apr 2010.

[24] J. J B N Van Berkel, J. W. Dallinga, G. M. Möller, et al. Development of accurate classification method based on the analysis of volatile organic compounds from human exhaled air. Journal of Chromatography B: Analytical Technologies in the Biomedical and Life Sciences, 861(1):101107, jan 2008.

[25] B. Walczak and D.L. Massart. Wavelets in Chemistry, volume 22. Elsevier Science B.V, nov 2000.

[26] Paul H. C. Eilers and Brian D. Marx. Flexible smoothing with B -splines and penalties. Statistical Science, 11(2):89-121, may 1996.

[27] Niels-Peter Vest Nielsen, Jens Michael Carstensen, and Jorn Smedsgaard. Aligning of single and multiple wavelength chromatographic profiles for chemoetric data analysis using correlation optimized warping. J. Chromatogr., A, 805(1-2):17-35, 1998.

[28] Agnieszka Baranska, Ettje Tigchelaar, Agnieszka Smolinska, et al. Profile of volatile organic compounds in exhaled breath changes as a result of gluten-free diet. Journal of breath research, 7(3):037104, sep 2013.

[29] J. R. de Haan, R. Wehrens, S. Bauerschmidt, et al. Interpretation of ANOVA models for microarray data using PCA. Bioinformatics, 23(2):184-190, jan 2007.

[30] J. R. de Haan, S. Bauerschmidt, R. C. van Schaik, et al. Robust ANOVA for microarray data. Chemometrics and Intelligent Laboratory Systems, 98(1):38-44, aug 2009.

[31] Paul Geladi, Hans Isaksson, Lennart Lindqvist, Svante Wold, and Kim Esbensen. Principal component analysis of multivariate images. Chemometrics and Intelligent Laboratory Systems, 5(3):209-220, mar 1989.

[32] Endre Anderssen, Knut Dyrstad, Frank Westad, and Harald Martens. Reducing over-optimism in variable selection by cross-model validation. Chemometrics and Intelligent Laboratory Systems, 84(1-2 SPEC. ISS.):69-74, dec 2006. 
[33] Lars Gidskehaug, Endre Anderssen, and Bjørn K. Alsberg. Cross model validation and optimisation of bilinear regression models. Chemometrics and Intelligent Laboratory Systems, 93(1):1-10, aug 2008.

[34] Massimo E Maffei, Jürg Gertsch, and Giovanni Appendino. Plant volatiles: production, function and pharmacology. Natural product reports, 28(8):1359-80, aug 2011.

[35] A Korpi, J Jarnberg, and A L Pasanen. Microbial volatile organic compounds. Crit Rev Toxicol, 39(2):139-193, jan 2009.

[36] E. Crespo, H. De Ronde, S. Kuijper, et al. Potential biomarkers for identification of mycobacterial cultures by proton transfer reaction mass spectrometry analysis. Rapid Communications in Mass Spectrometry, 26(6):679-685, mar 2012.

[37] Lieuwe D. J. Bos, Peter J. Sterk, Marcus J. Schultz, L Hofmann, and S Ulbricht. Volatile Metabolites of Pathogens: A Systematic Review. PLoS Pathogens, 9(5):e1003311, may 2013.

[38] Wojciech Filipiak, Andreas Sponring, Maria M. Baur, et al. Characterization of volatile metabolites taken up by or released from Streptococcus pneumoniae and Haemophilus influenzae by using GC-MS. Microbiology (United Kingdom), 158(12):3044-3053, dec 2012.

[39] Wojciech Filipiak, Andreas Sponring, Maria Magdalena Baur, et al. Molecular analysis of volatile metabolites released specifically by staphylococcus aureus and pseudomonas aeruginosa. BMC Microbiology, 12(1):113, jun 2012.

[40] George Preti, Erica Thaler, C. William Hanson, et al. Volatile compounds characteristic of sinus-related bacteria and infected sinus mucus: Analysis by solid-phase microextraction and gas chromatography-mass spectrometry. Journal of Chromatography B: Analytical Technologies in the Biomedical and Life Sciences, 877(22):2011-2018, jul 2009.

[41] W Carroll, W Lenney, T S Wang, et al. Detection of volatile compounds emitted by Pseudomonas aeruginosa using selected ion flow tube mass spectrometry. Pediatric Pulmonology, 39(5):452-456, may 2005.

[42] Heather D Bean, Jean-Marie D Dimandja, and Jane E Hill. Bacterial volatile discovery using solid phase microextraction and comprehensive two-dimensional gas chromatographytime-offlight mass spectrometry. Journal of chromatography. B, Analytical technologies in the biomedical and life sciences, 901:41-46, jul 2012.

[43] Nils Kunze, Julia Göpel, Martin Kuhns, et al. Detection and validation of volatile metabolic patterns over different strains of two human pathogenic bacteria during their growth in a complex medium using multi-capillary column-ion mobility spectrometry (MCC-IMS). Applied Microbiology and Biotechnology, 97(8):3665-3676, apr 2013.

[44] Robin Michael Statham Thorn, Darren Michael Reynolds, and John Greenman. Multivariate analysis of bacterial volatile compound profiles for discrimination between selected species and strains in vitro. Journal of Microbiological Methods, 84(2):258-264, feb 2011.

[45] H. Kiviranta, A. Tuomainen, M. Reiman, et al. Qualitative identification of volatile metabolites from two fungi and three bacteria species cultivated on two media. Central European Journal of Public Health, 6(4):296-299, nov 1998.

[46] Matthias Lechner, Herbert Tilg, and Josef Rieder. Analysis of volatile compounds emitted by the Helicobacter pylori reference strain NCTC 11637 in vitro. Helicobacter, 11(1):66, feb 2006.

[47] David S. Armstrong, Keith Grimwood, John B. Carlin, et al. Bronchoalveolar lavage or oropharyngeal cultures to identify lower respiratory pathogens in infants with cystic fibrosis. Pediatric Pulmonology, 21(5):267-275, may 1996.

[48] Ana S M Afonso, Katia M C Verhamme, Miriam C J M Sturkenboom, and Guy G O Brusselle. COPD in the general population: Prevalence, incidence and survival. Respiratory Medicine, 105(12):1872-1884, dec 2011.

[49] Tetyana Zakharkina, A. Rembert Koczulla, Olga Mardanova, Akira Hattesohl, and Robert Bals. Detection of microorganisms in exhaled breath condensate during acute exacerbations of COPD. Respirology, 16(6):932-938, aug 2011. 
[50] Jonathan Messika, Fatma Magdoud, Olivier Clermont, et al. Pathophysiology of Escherichia coli ventilator-associated pneumonia: Implication of highly virulent extraintestinal pathogenic strains. Intensive Care Medicine, 38(12):2007-2016, dec 2012.

[51] Mona Syhre, Jennifer M Scotter, and Stephen T Chambers. Investigation into the production of 2-Pentylfuran by Aspergillus fumigatus and other respiratory pathogens in vitro and human breath samples. Medical mycology : official publication of the International Society for Human and Animal Mycology, 46(May):209-215, may 2008.

[52] J. N. Labows, K. J. McGinley, G. F. Webster, and J. J. Leyden. Headspace analysis of volatile metabolites of Pseudomonas aeruginosa and related species by gas chromatography-mass spectrometry. Journal of Clinical Microbiology, 12(4):521-526, oct 1980.

[53] James M. Zechman, Scott Aldinger, and John N. Labows. Characterization of pathogenic bacteria by automated headspace concentration-gas chromatography. Journal of Chromatography B: Biomedical Sciences and Applications, 377(C):49-57, jan 1986.

[54] Sasidhar Maddula, Lars Mathias Blank, Andreas Schmid, and J??rg Ingo Baumbach. Detection of volatile metabolites of Escherichia coli by multi capillary column coupled ion mobility spectrometry. Analytical and Bioanalytical Chemistry, 394(3):791-800, jun 2009.

[55] Melanie Jünger, Wolfgang Vautz, Martin Kuhns, et al. Ion mobility spectrometry for microbial volatile organic compounds: A new identification tool for human pathogenic bacteria. Applied Microbiology and Biotechnology, 93(6):2603-2614, mar 2012.

[56] Michael Bunge, Nooshin Araghipour, Tomas Mikoviny, et al. On-Line Monitoring of Microbial Volatile Metabolites by Proton Transfer On-Line Monitoring of Microbial Volatile Metabolites by Proton. Appl. Environ. Microbiol., 74(7):2179-2186, apr 2008.

[57] J Julák, E Stránská, E Procházková-Francisci, and V Rosová. Blood cultures evaluation by gas chromatography of volatile fatty acids. Medical science monitor : international medical journal of experimental and clinical research, 6(3):605-10, 2000.

[58] Malina K. Storer, Kim Hibbard-Melles, Brett Davis, and Jenny Scotter. Detection of volatile compounds produced by microbial growth in urine by selected ion flow tube mass spectrometry (SIFT-MS). Journal of Microbiological Methods, 87(1):111-113, oct 2011.

[59] M O'Hara and C.A. Mayhew. A preliminary comparison of volatile organic compounds in the headspace of cultures of Staphylococcus aureus grown in nutrient, dextrose and brain heart bovine broths measured using a proton transfer reaction mass spectrometer. Journal of breath research, 3(2):027001, jun 2009.

[60] Jiangjiang Zhu, Heather D. Bean, Yin-Ming Ming Kuo, and Jane E. Hill. Fast detection of volatile organic compounds from bacterial cultures by secondary electrospray ionization-mass spectrometry. Journal of Clinical Microbiology, 48(12):4426-4431, dec 2010.

[61] Jiangjiang Zhu and Jane E Hill. Detection of Escherichia coli via VOC pro fi ling using secondary electrospray ionization-mass spectrometry ( SESI-MS ). Food Microbiology, 34:8-13, 2013.

[62] E. Crespo, S. M. Cristescu, H. de Ronde, et al. Proton Transfer Reaction Mass Spectrometry detects rapid changes in volatile metabolite emission by Mycobacterium smegmatis after the addition of specific antimicrobial agents. Journal of Microbiological Methods, 86(1):8-15, jul 2011.

[63] Charlotte M H H T Robroeks, Joep J B N van Berkel, Jan W. Dallinga, et al. Metabolomics of volatile organic compounds in cystic fibrosis patients and controls. Pediatric research, 68(1):7580, jul 2010.

[64] Amy J Scott-Thomas, Mona Syhre, Philip K Pattemore, et al. 2-Aminoacetophenone as a potential breath biomarker for Pseudomonas aeruginosa in the cystic fibrosis lung. BMC pulmonary medicine, 10(1):56, jan 2010.

[65] David Smith, Patrik Španěl, Francis J Gilchrist, et al. Hydrogen cyanide, a volatile biomarker of $<\mathrm{i}>$ Pseudomonas aeruginosa $</ \mathrm{i}>$ infection. Journal of Breath Research, 7(4):044001, dec 2013.

[66] Jiangjiang Zhu, Heather D Bean, Matthew J Wargo, Laurie W Leclair, and Jane E Hill. Detecting bacterial lung infections: in vivo evaluation of in vitro volatile fingerprints. Journal of breath research, 7(1):16003, mar 2013. 
CHAPTER 7

Exposure to genotoxic compounds alters in vitro cellular VOC excretion

R Fijten, A. Smolinska, Q. Shi, D. Pachen, J. Dallinga, A. Boots, F. van Schooten J Breath Research, 2017. Accepted for publication 


\subsection{Abstract}

Genotoxic carcinogens significantly damage cells and tissues by targeting macromolecules such as proteins and DNA, but their mechanisms of action and effects on human health are diverse. Consequently, determining the amount of exposure to a carcinogen and its cellular effects is essential, yet difficult.

The aim of this manuscript was to investigate the potential of detecting alterations in Volatile Organic Compounds (VOCs) profiles in the in vitro headspace of pulmonary cells after exposure to the genotoxic carcinogens cisplatin and benzo[a]pyrene. Both carcinogens were added to the cell medium for 24 hours. The headspace in the culture flask was sampled to measure the VOC content using gas chromatography time of flight mass spectrometry. Eight cisplatin-specific VOCs and six benzo[a]pyrene-specific VOCs were discriminatory between treated and non-treated cells. Since the in vivo biological effects of both genotoxic compounds are well-defined, the origin of the identified VOCs could potentially be traced back to common cellular processes including cell cycle pathways, DNA damage and repair. These results have proven that exposing lung cells to genotoxins alters headspace VOC profiles, suggesting that it might be possible to monitor VOC changes in vivo to study drug efficacy or exposure to different pollutants. In conclusion, this study emphasizes the innovative potential of in vitro VOCs experiments to determine their in vivo applicability and discover their endogenous origin. Genotoxic carcinogens significantly damage cells and tissues by targeting macromolecules such as proteins and DNA, but their mechanisms of action and effects on human health are diverse. Consequently, determining the amount of exposure to a carcinogen and its cellular effects is essential, yet difficult. The aim of this manuscript was to investigate the potential of detecting alterations in Volatile Organic Compounds (VOCs) profiles in the in vitro headspace of pulmonary cells after exposure to the genotoxic carcinogens cisplatin and benzo[a]pyrene. Both carcinogens were added to the cell medium for 24 hours. The headspace in the culture flask was sampled to measure the VOC content using gas chromatography time of flight mass spectrometry. Eight cisplatin-specific VOCs and six benzo[a]pyrenespecific VOCs were discriminatory between treated and non-treated cells. Since the in vivo biological effects of both genotoxic compounds are well-defined, the origin of the identified VOCs could potentially be traced back to common cellular processes including cell cycle pathways, DNA damage and repair.

These results have proven that exposing lung cells to genotoxins alters headspace VOC profiles, suggesting that it might be possible to monitor VOC changes in vivo to study drug efficacy or exposure to different pollutants. In conclusion, this study emphasizes the innovative potential of in vitro VOCs experiments to determine their in vivo applicability and discover their endogenous origin.

\subsection{Introduction}

Genotoxic carcinogens damage cells and tissues by covalent binding to the DNA, either directly or indirectly [1], leading to P53 activation that results in modulation of a variety of cellular pathways including apoptosis, DNA damage and DNA repair [1]. 
One of the most studied sources of genotoxic carcinogens is tobacco smoke. It contains over 700 carcinogenic compounds including a large group of polycyclic aromatic hydrocarbons $(\mathrm{PAH})[2]$, which have been implicated in a variety of cancers including lung cancer [3]. Benzo[a]pyrene $(\mathrm{B}[\mathrm{a}] \mathrm{P})$ is a well-studied $\mathrm{PAH}$ and its metabolites are very mutagenic and carcinogenic by forming DNA adducts or distortion of the helix structure [4]. Currently, 1-hydroxypyrene, a metabolite of the PAH pyrene, is used to quantify the level of exposure to PAHs [5]. It is, however, not specific for $\mathrm{B}[\mathrm{a}] \mathrm{P}$ exposure as the ratio between pyrene and $\mathrm{B}[\mathrm{a}] \mathrm{P}$ varies in airborne mixtures $[6]$. Additionally, determining 1-hydroxypyrene in urine does not only measure exposure to pyrene, but also its biotransformation, absorption and excretion [7]. It therefore does not allow accurate assessment of the risk of lung cancer due to persistent contact with PAHs such as B[a]P through for example tobacco smoke. Consequently, assessing of $\mathrm{B}[\mathrm{a}] \mathrm{P}$ exposure by applying other biomarkers is of interest, in particular to determine the risk of developing lung cancer [4, 8]].

Advanced lung cancer is usually treated with cisplatin-based chemotherapy [9]. Interestingly, cisplatin is also a genotoxic carcinogen that forms adducts with the DNA [10]. Similar to B[a]P, cisplatin-induced DNA damage affects proliferation, differentiation, DNA repair and apoptosis among other related pathways in a way that is harmful to the health of the target cell. As the efficacy of cisplatin treatment depends on successfully affecting these pathways in cancer cells, it is of interest to find biomarkers of cancer cell responses to cisplatin exposure. Interestingly, metabolic and volatile changes in the in vitro cell metabolism of cells treated with cisplatin have already been demonstrated [11, 12], suggesting a role for (volatile) metabolites as biomarkers to monitor cisplatin treatment $[11,12]$.

To determine whether cells excrete specific volatile organic compounds (VOCs) in their headspace upon exposure to a genotoxic carcinogen, we analyzed VOCs excreted by lung carcinoma cells after cisplatin exposure and by lung epithelial cells after B[a]P exposure.

\subsection{Materials and Methods}

\subsubsection{Cell culture}

The A549 human carcinoma cell line (ATCC CCL-185) was cultured in RPMI 1640 medium (Without Phenol Red; Gibco, Invitrogen), supplemented with $5 \%$ heat inactivated Fetal Bovine Serum (Gibco, Invitrogen). The BEAS-2B lung epithelial cells (ATCC CRL-9609) were cultured in DMEMF-Dulbeccos Modified Eagles medium (DMEMF-12, Gibco, Invitrogen), supplemented with 1\% penicillin/streptomycin, 15 $\mathrm{\mu g} / \mathrm{ml}$ bovine pituitary extract, $0.5 \mathrm{ng} / \mathrm{ml}$ bovine serum albumin (Invitrogen, Breda, The Netherlands), $10 \mathrm{ng} / \mathrm{ml}$ epidermal growth factor (Merck Millipore, Darmstadt, Germany), $10 \mathrm{ng} / \mathrm{ml}$ cholera-toxin (list Biological Laboratories, Inc., Campbell, California), $5 \mathrm{~g} / \mathrm{ml}$ insulin, $5 \mu \mathrm{g} / \mathrm{ml}$ transferrin and $0.1 \mu \mathrm{M}$ dexamethasone (SigmaAldrich, St. Louis, MO, USA). Both cell lines were cultured in Corning cell culture flasks (Corning Incorporated, New York, USA) to $80-90 \%$ confluence at $37{ }^{\circ} \mathrm{C}$ and $5 \%$ CO2. Flasks with cells were randomly divided into a control and exposure group 
each containing 12 or 16 biological replicates for the A549 and BEAS-2B experiments respectively.

\subsubsection{Exposure}

Upon reaching 80-90\% confluency, the medium was replaced by freshly prepared medium containing cisplatin dissolved in phosphate buffer saline (CAS no. 1566327-1; >96\% purity; Sigma-Aldrich, St. Louis, USA) for the A549 cells and B[a]P dissolve in dimethyl sulfoxide (CAS no. 50-32-8; purity >96\%; Sigma-Aldrich, St. Louis, USA) for the BEAS-2B cells. For both exposures, the appropriate concentration was chosen based on an MTT proliferation assay [13]. For cisplatin, the GI50 concentration of $50 \mu \mathrm{M}$ (at which a growth inhibition of $50 \%$ is achieved) was chosen, whereas for $\mathrm{B}[\mathrm{a}] \mathrm{P}$, a non-lethal dose of $1 \mu \mathrm{M}$ was chosen (where $100 \%$ of cells were still alive). Both cell lines were exposed for 24 hours to their respective genotoxic carcinogen.

\subsubsection{Headspace sampling set-up}

The VOCs excreted by the A549 cells exposed to cisplatin were collected as previously described by Baranska et al. [14]. After using this prototype in vitro measurement setup, several modifications were added to the system to improve heat distribution and reduce the influence of fluctuating temperature levels. The headspace of the $\mathrm{B}[\mathrm{a}] \mathrm{P}-$ treated BEAS-2B cells was sampled using this new system (Figure 7.1). Four flasks were sampled simultaneously and were kept inside an incubator at $37^{\circ} \mathrm{C}$ throughout the sampling procedure. The flasks were connected to a gas cylinder (pressure: 150 bar) containing a purified mixture of N2 (75\%), O2 (20\%) and CO2 (5\%) by tubing and a flow regulator. Each flask had an outlet that directed the headspace to a stainless steel two-bed sorption tube filled with carbograph 1TD/Carbopack X (Markes International, Llantrisant, Wales, UK) to collect the VOCs.

For the cisplatin exposure of the A549 cells, the headspace was sampled for 1 hour after 23 hours of exposure at a flow rate of $25 \mathrm{ml} / \mathrm{min}$. For the BEAS-2B cells exposed to $\mathrm{B}[\mathrm{a}] \mathrm{P}$, this was done continuously during the 24 hours of exposure at a rate of 4 $\mathrm{ml} / \mathrm{min}$ to potentially increase the quantity and number of VOCs trapped.

\subsubsection{Gas chromatography mass spectrometry analysis}

After collection of the VOCs from the headspace, the thermal desorption tubes were measured by gas chromatography time of flight mass spectrometry (GC-tof-MS).

The desorption tubes were placed inside the TD100 automated thermal desorber (Markes International, Llantrisant, Wales, UK) and heated to 350C to release the VOCs from the tubes. $90 \%$ of the released compounds were trapped on a second, identical tube for potential repeated analysis in case of failure of the measurement by GC-MS. Ten percent was trapped at a cold trap at 5C, from where it was injected into the GC-column at a temperature of 300C. The VOCs were subsequently separated by capillary gas chromatography Trace 1300GC (Thermo Fisher Scientific, Waltham, Massachusetts; column: RTX-5ms, $30 \mathrm{~m} 0.25 \mathrm{~mm} \mathrm{5 \%}$ diphenyl, $95 \%$ 


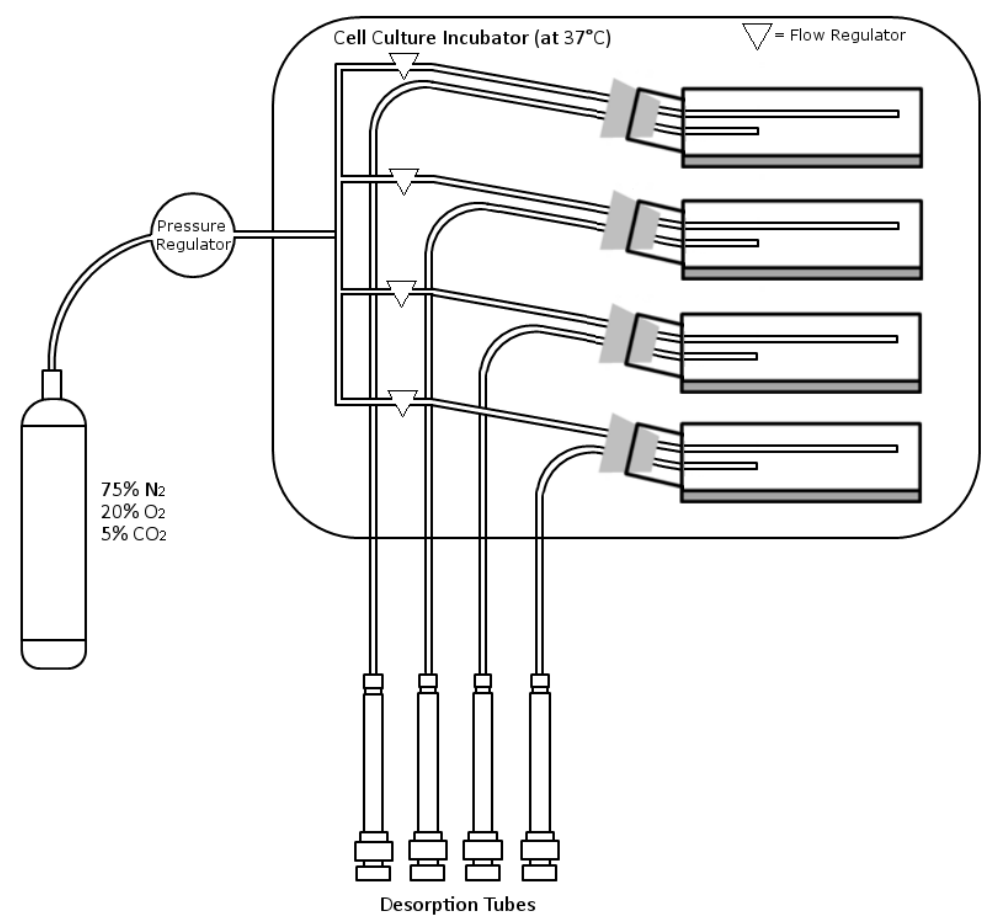

Figure 7.1: In vitro sampling of the headspace of BEAS-2B cells exposed to $\mathrm{B}[\mathrm{a}] \mathrm{P}$ for 24 hours.

dimethylsiloxane, film thickness $1 \mu \mathrm{m}$ ). The temperature of the gas chromatograph was programmed as follows: $40{ }^{\circ} \mathrm{C}$ for 5 minutes then raised with $10^{\circ} \mathrm{C} / \mathrm{min}$ until a final maximum temperature of $270^{\circ} \mathrm{C}$. The final temperature was maintained for 5 minutes. Time-of-flight mass spectrometry (tof-MS; Bench TOF-dx Almsco International, Llantrisant, Wales, UK)) was used to detect and identify components available in the samples. Electron ionization mode was set at $70 \mathrm{eV}$ and the mass range $\mathrm{m} / \mathrm{z} 35-$ 350 was measured. Sample frequency of the mass spectrometer was set to 5 scans/sec and analysis run time to $33 \mathrm{~min}$. For each sample, a chromatogram was obtained containing $\mathrm{m} / \mathrm{z}$ values for each measured compound.

\subsubsection{Data preprocessing}

After chromatograms were obtained, they were preprocessed as described earlier [15] to remove any non-biological variation. In short, the data were log transformed [16] and subsequently denoised by a Daubechies wavelet with two levels of compression [17]. Baseline correction and normalization were done by B-splines with asymmetric least squares smoothing [18] and probabilistic quotient normalization [19], respectively. Peak picking was carried out and peaks for the same compound were aligned across samples by correlation analysis of the mass spectra. Data analysis After data preprocessing, two comparisons were tested: 
1. Cisplatin exposed A549 cells vs. control A549 cells

2. Benzo[a]pyrene exposed BEAS-2B cells vs. control BEAS-2B cells

For both comparisons, a model was created to separate the controls from exposed cells using Random Forests (RF) [20]. The out-of-bag error and the importance of specific VOCs to the model were calculated. This compound importance was used to select a group of discriminatory VOCs. A final RF model was created for each comparison containing only this subset of discriminatory VOCs. The final results are displayed as principal component analysis (PCA) score plot obtained from proximities of the final RF model [21].

After selection of the discriminatory VOCs, univariate significance testing was performed on the individual discriminatory VOCs by means of the Mann Whitney-U test in combination with multiple-testing correction by False Discovery Rate (FDR). An adjusted p-value of 0.05 was considered significant. Putative chemical identification of VOCs The data analysis resulted in two lists of discriminatory VOCs, one for each exposure. The retention time and mass spectrum of each peak were examined using the National Institute of Standard and Technology (NIST) library in conjunction with an in-house library with pure compounds. As a final validation, an experienced mass spectrometrist evaluated and confirmed the identities of the VOCs.

\subsection{Results}

Headspace air from 56 samples was collected and analyzed for the presence of VOCs. The samples were divided into four groups: 12 cisplatin-exposed A549 samples, 12 A549 controls, 16 B[a]P-exposed BEAS-2B samples and 16 BEAS-2B controls. Predictive modelling resulted in the identification of two groups of VOCs, one for each exposure. For the cisplatin, 8 VOCs were identified as discriminatory between A549 cells with and without exposure to this carcinogen. The model, of which the corresponding PCA scores plot is displayed in Figure 7.2(a), achieved an overall prediction of $83.3 \%$. A subset of 6 VOCs was identified for the BEAS-2B cells exposed to B[a]P with an overall prediction of $65.6 \%$. The PCA scores plot associated with these six VOCs is shown in Figure 7.2(b).

The putative chemical identities including corresponding CAS numbers of the discriminatory VOCs are listed in Table 7.S1 and Table 7.S2 for cisplatin and B[a]P exposure respectively. Nonanal was the only compound found to be discriminatory for both exposures. The impact of each VOC on the discriminatory model is visualized in Figure 7.3(a) and (b) for the cisplatin and $\mathrm{B}[\mathrm{a}] \mathrm{P}$ exposures, respectively. In the figures, the length of the bar indicates the relative importance of a VOC to distinguish exposed from unexposed cells, whereas its direction is an indication of the change in the relative concentration of each VOC. A positive bar indicates an increased relative concentration of a VOC, whereas a negative bar indicates a lowered level of the VOC in the exposed cells in comparison to the controls. For cisplatin, the levels of three VOCs were lowered, whereas the other five occurred in higher concentrations upon exposure to the carcinogen. Ethenylbenzene had the highest negative importance, whereas diethyl ether had the highest positive importance in the model. Boxplots 

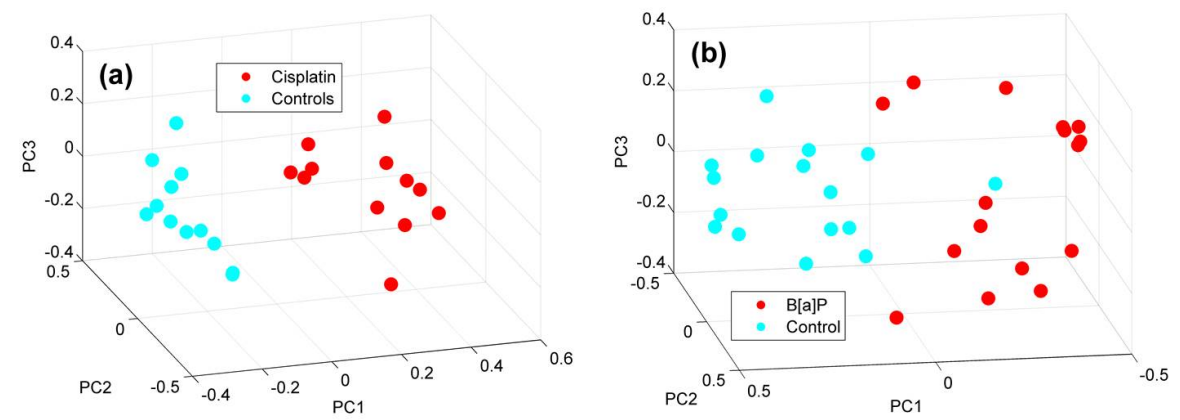

Figure 7.2: PCA score plot of proximities calculated by the respective Random Forests models for (a) cisplatin exposure and (b) benzo[a]pyrene exposure. Red $=$ exposed cells; Blue $=$ controls.

of the relative concentrations of each VOC can be found in Figures 7.S1 and 7.S2 in the supplementary materials. None of the identified VOCs were significant after univariate testing in combination with FDR correction.

After $\mathrm{B}[\mathrm{a}] \mathrm{P}$ exposure, the concentration of o-xylene, nonanal, decanal and one unknown VOC were reduced in exposed cells. Isopropyl alcohol and octadecane had a higher concentration after exposure. In addition, univariate significance testing in combination with FDR correction was performed where only the unknown VOC was independently significant $(\mathrm{p}=0.04)$.
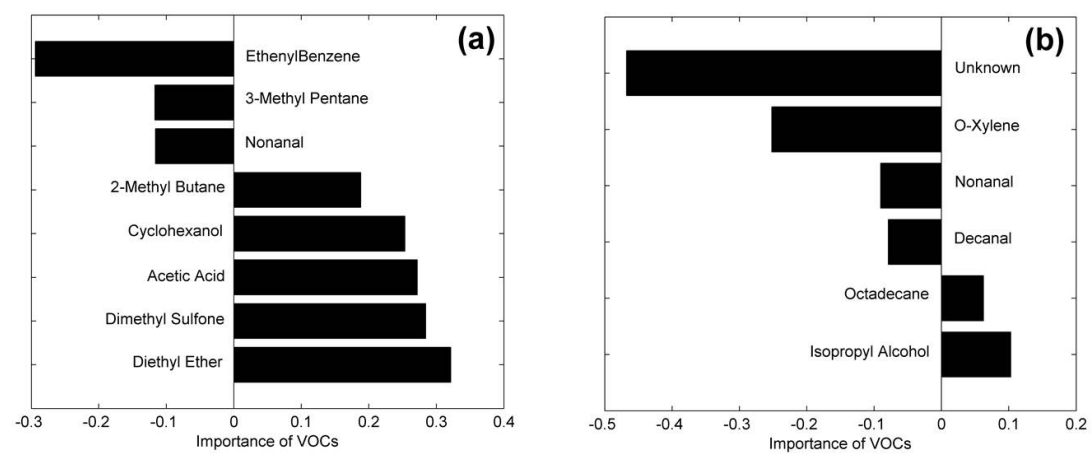

Figure 7.3: Importance of each selected VOC to the discriminatory model identified after both exposures. The length of the bar indicates the relative importance of a variable to the model. A negative value indicates a reduced concentration excreted by the exposed cells compared to the controls; a positive value indicates an increased concentration for exposed cells vs. controls.(a) Cisplatin exposure; (b) $\mathrm{B}[\mathrm{a}] \mathrm{P}$ exposure. 


\subsection{Discussion \& Conclusion}

The aim of this study was to identify VOCs specific for in vitro exposure to either benzo[a]pyrene or cisplatin. In both cases, a subset of VOCs was identified that could discriminate between exposed and non-exposed cells. Univariate significance testing of the individual compounds did not yield significant outcomes, indicating that a profile of volatiles, rather than an individual one, is needed to distinguish between exposed and non-exposed cells.

The molecular mechanisms of action of the two genotoxic carcinogens used in the present study, i.e. cisplatin and $\mathrm{B}[\mathrm{a}] \mathrm{P}$, are well established, providing information about the possible origin of the discriminatory VOCs. Cisplatin is a platinum-based compound used as chemotherapy for a variety of cancers including lung cancer [9]. It is actively transported into the cell where it is hydrolyzed. This hydrolyzed product can then bind directly to the DNA, causing DNA damage and blocking cell division [10]. Pathways involved in cisplatin-induced toxicity are DNA damage and repair, mismatch repair, P53 activation, cell cycle arrest and finally apoptosis . In case of cancer cells, a higher level of oxidative stress is observed in comparison to normal cells, which in combination with the effects of cisplatin results in apoptosis $[10,22]$. B $[\mathrm{a}] \mathrm{P}$ in itself contains no reactive groups but is activated by cytochrome P450 enzymes (especially CYP1A1) into the reactive metabolite diol epoxide metabolite (BPDE). BPDE reacts with DNA to form covalent DNA adducts [23] that cause DNA damage, cell cycle arrest and apoptosis, processes that are also evoked by cisplatin. Additionally, we have previously shown that the BEAS-2B cells are responsive to the dose used in our study since cytochrome P450 (CYP1A1) gene expression and its activity (ethoxyresorufin-O-deethylase activity) were elevated up to 20 times [24, 25].

Considering the modulation of these pathways, it is likely that they are associated with the discriminatory VOCs excreted in the headspace upon exposure. For cisplatin, the use of the GI50 concentration has resulted in production of cell death related VOCs, whereas the non-lethal exposure of $\mathrm{B}[\mathrm{a}] \mathrm{P}$ has led to more subtle changes in underlying $\mathrm{B}[\mathrm{a}] \mathrm{P}$-related pathways.

The appearance of the (methylated) alkanes octadecane, 3-methylpentane and 2methylbutane may be explained by the induction of lipid peroxidation and oxidative stress by both triggers [26-28]. Aldehydes such as nonanal and decanal are produced as by-products of oxidative metabolism upon the induction of cytochrome P450 enzymes due to $\mathrm{B}[\mathrm{a}] \mathrm{P}$ exposure. Alternatively, they are produced as a result of $\mathrm{B}[\mathrm{a}] \mathrm{P}$ or cisplatin induced alterations in normal physiological processes such as including signal transduction, proliferation and gene regulation [28]. Nonanal is especially of interest because it was found in both the cisplatin and $\mathrm{B}[\mathrm{a}] \mathrm{P}$ exposures and is confirmed by literature for cisplatin exposure [12], hinting at a potential use as an exposure marker to genotoxic carcinogens. The occurrence of isopropyl alcohol upon exposure to $\mathrm{B}[\mathrm{a}] \mathrm{P}$ is due to its production during ketogenesis [29], which itself results from an altered fatty acid metabolism and enhanced lipid peroxidation [30] due to exposure to genotoxic chemicals [31]. The reduced occurrence of the aromatic hydrocarbons ethenylbenzene and o-xylene upon exposure to both triggers is difficult to interpret as both VOCs are considered exogenous [32, 33]. Contamination of these compounds by the materials used in the set-up seems unlikely as we used a pure gas mixutre and 
the system-related VOCs would be present in each sample in a similar amount, thus not serving as discriminatory. A potential endogenous source of these two compounds is unknown, yet once present they can be further metabolized by human cells [34]. This suggests that the carcinogenic exposures may influence the biotransformation of these aromatic hydrocarbons, resulting in a lower concentration after exposure to either $\mathrm{B}[\mathrm{a}] \mathrm{P}$ or cisplatin. Finally, acetic acid is produced by gut bacteria in an in vivo situation, after which it is taken up into the blood and excreted in the exhaled breath $[35,36]$. However, this does not apply to our in vitro situation where no gut bacteria are present. In this case, the most likely source of acetic acid us the protonation is the formation of acetic acid, which forms an equilibrium with acetate. Acetate in turn is formed as a result of hydrolysis of acetylated metabolites and deacetylation of histones and proteins [37, 38]. Research has shown that phosphorylation and acetylation of histones occurs after cisplatin exposure [39], suggesting a lowering in the acetate concentration and thus the concentration of acetic acid in the headspace as was reported in the current study. The source of the remaining two compounds (dimethyl sulfone and diethyl ether) remains unclear. However, it has to be noted that these VOCs were found in an in vitro experiment, and may not translate to an in vivo situation.

In recent years, the number of studies applying mammalian in vitro experiments using different approaches to detect VOCs in headspace as indicator of cellular processes has increased. The majority of these studies can be classified as a proof-of-principle approach where VOCs excreted by the cells are measured in the headspace [40-45]. A different angle was taken in several other studies that compared cell lines with different characteristics, such as presence or absence of oncogenes, to link VOCs in headspace to these specific characteristics [46-51]. However, since these different characteristics can alter a variety of pathways, it is still difficult to pinpoint the exact pathway associated with a specific VOC. A third option is to use a trigger to elicit a certain, often well-understood, cellular response or to influence one specific pathway. This approach was taken for instance by Pyo et al. [12], who exposed A549 cells to different concentrations of cisplatin showing that VOCs profiles are altered upon exposure to cisplatin.

In the present study, by applying two genotoxic carcinogens as triggers, associations were made between the excreted VOCs and certain pathways provoked by these triggers. Indeed, finding discriminatory VOC profiles specific for either one of the two carcinogenic exposures indicates that cellular processes are altered, resulting in detectable changes in excreted VOCs. These in vitro results might give some insight towards the VOC pattern that might be present in the exhaled breath of individuals exposed to cisplatin or $\mathrm{B}[\mathrm{a}] \mathrm{P}$. However, there is still a large gap between in vivo and in vitro experiments due to for instance the fact that cisplatin is not delivered directly to the cells in an in vivo situation. Additionally, the artificial in vitro environment is not representative of an in vivo experiment, as reflected by for instance the hyperoxic conditions in the cell culture incubator [52]. Therefore, different VOCs may be found in an in vivo experiment, making it more difficult to pinpoint the exact metabolic origin of the discriminatory volatiles. This translational gap between in vitro and in vivo research may be diminished by using primary cells.

Although observations deviating from (exposed) cells are interesting, they always 
refer to indirect associations and provide circumstantial proof of the underlying pathways involved. Direct relations can only be found by measuring headspace VOCs after manipulation of designated pathways, using for instance molecular techniques to up- or downregulate specific pathways using RNAi or CRISPR-Cas9 [53, 54]. Such an approach could pinpoint the exact effect of that pathway on the production or uptake of unique VOCs. Another way of demonstrating direct associations is to combine headspace VOCs analysis with other omics, including transcriptomics and metabolomics, to find relations between genes, metabolites and VOCs. This methodology, being the most informative and optimal one, has not been done before but holds great promise for the future.

In conclusion, this study has demonstrated that exposure to genotoxic carcinogens causes a change in the profile of excreted headspace VOCs in vitro. Especially nonanal seems a viable marker for exposure to genotoxic carcinogens as it was found in both exposures and confirmed by literature [12]. The future direction of in vitro VOCs research should focus on determining the endogenous sources of VOCs, either by selective manipulation of underlying pathways or by adding other omics to the equation. A collective effort is needed from the breath community to gain the desired insights into the origin and biological meaning of in vitro VOCs.

\subsection{Supplementary Materials}

Table 7.S1: Identified VOCs for the A549 cells with cisplatin exposure. The name of the compound, its CAS number, $\mathrm{m} / \mathrm{z}$ of the parent ion, the retention time at which they were found and the chemical formula are displayed.

\begin{tabular}{lllll}
\hline VOC & CAS number & $\mathrm{m} / \mathrm{z}$ & $\begin{array}{l}\text { Retention } \\
\text { time }\end{array}$ & Chemical formula \\
\hline Diethyl Ether & $60-29-7$ & 74 & 2.96 & (C2H5)2O \\
Dimethyl Sulfone & $67-71-0$ & 94 & 3.52 & $\mathrm{C} 2 \mathrm{H} 6 \mathrm{O} 2 \mathrm{~S}$ \\
Acetic Acid & $64-19-7$ & 60 & 4.28 & $\mathrm{CH}$ COOH \\
2-Methyl Butane & $78-78-4$ & 72 & 4.75 & $\mathrm{C} 5 \mathrm{H} 12$ \\
3-Methyl Pentanal & $15877-57-3$ & 100 & 7.88 & $\mathrm{C} 6 \mathrm{H} 12 \mathrm{O}$ \\
Ethenylbenzene & $9003-53-6$ & 104 & 13.01 & $\mathrm{C} 8 \mathrm{H} 8$ \\
Nonanal & $124-19-6$ & 142 & 16.97 & $\mathrm{C} 9 \mathrm{H} 18 \mathrm{O}$ \\
Cyclohexanol & $108-93-0$ & 100 & 12.80 & $\mathrm{C} 6 \mathrm{H} 12 \mathrm{O}$
\end{tabular}


Table 7.S2: Identified VOCs for the BEAS-2B cells exposed to B[a]P. The name of the compound, its CAS number, $\mathrm{m} / \mathrm{z}$ of the parent ion, the retention time at which they were found and the chemical formula are displayed. * supposed parent ion.

\begin{tabular}{lllll}
\hline VOC & CAS number & $\mathrm{m} / \mathrm{z}$ & $\begin{array}{l}\text { Retention } \\
\text { time }\end{array}$ & Chemical formula \\
\hline Isopropyl Alcohol & $67-63-0$ & 60 & 2.64 & $\mathrm{C} 3 \mathrm{H} 8 \mathrm{O}$ \\
O-Xylene & $95-47-6$ & 106 & 12.91 & $\mathrm{C} 8 \mathrm{H} 10$ \\
Nonanal & $124-19-6$ & 142 & 16.85 & $\mathrm{C} 9 \mathrm{H} 18 \mathrm{O}$ \\
Decanal & $112-31-2$ & 156 & 18.54 & $\mathrm{C} 10 \mathrm{H} 20 \mathrm{O}$ \\
Octadecane & $593-45-3$ & 254 & 25.77 & $\mathrm{C} 18 \mathrm{H} 38$ \\
Unknown & $\mathrm{NA}$ & $282^{*}$ & 14.63 & $\mathrm{NA}$
\end{tabular}
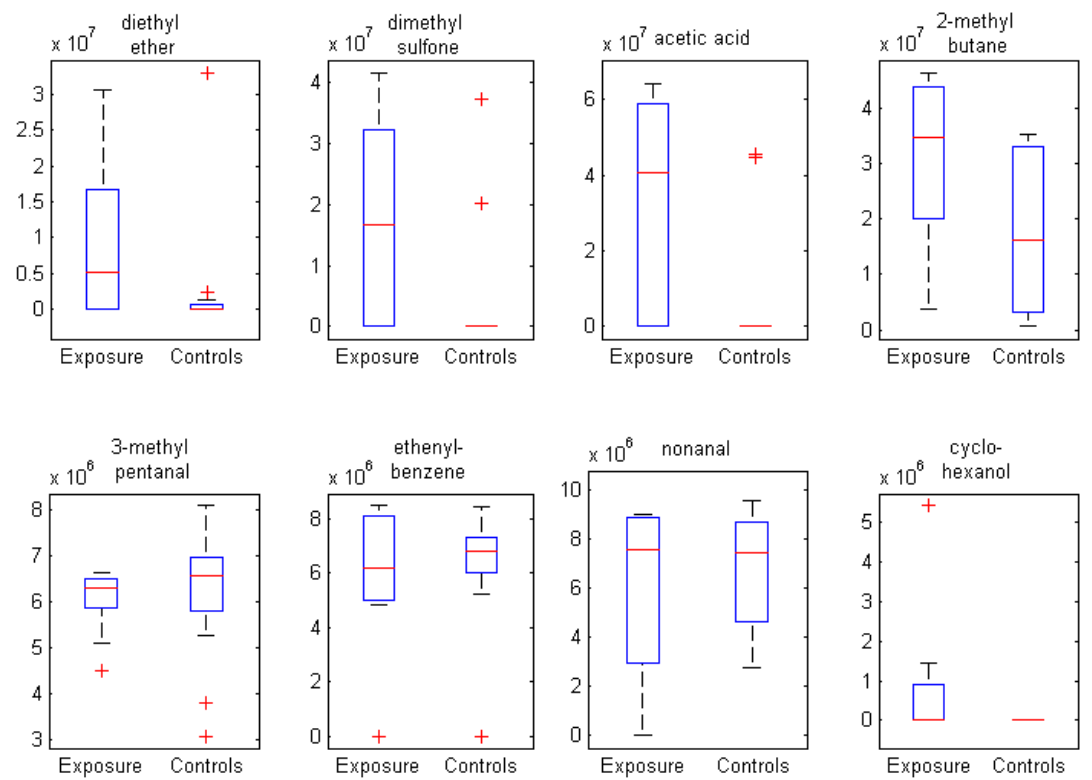

Figure 7.S1: Boxplots of the relative concentrations of each discriminatory VOC after cisplatin exposure. The red line indicates the median relative concentration and the blue boxes demonstrate the range from the first to the third quartile. The whiskers indicate the maximum spread of the data, with outliers denoted as a red "+" sign. 

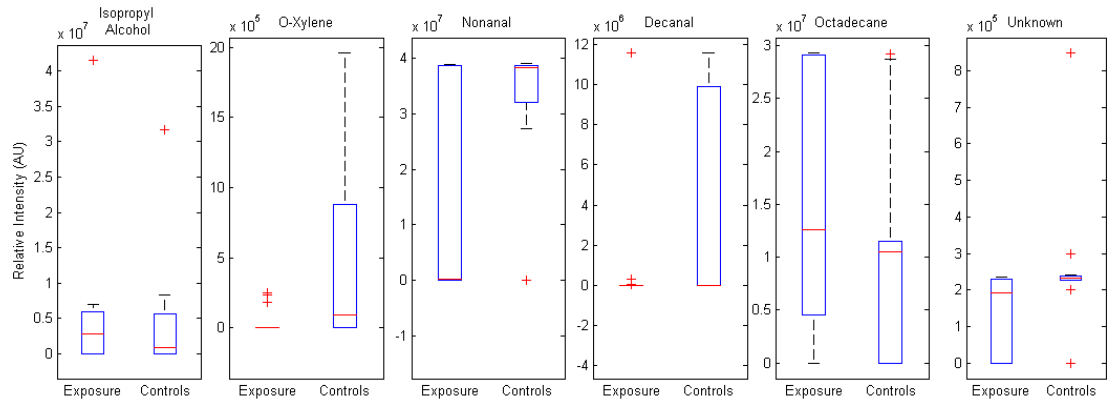

Figure 7.S2: Boxplots of the relative concentrations of each discriminatory VOC after $\mathbf{B}[\mathbf{a}] \mathbf{P}$ exposure. The red line indicates the median relative concentration and the blue boxes demonstrate the range from the first to the third quartile. The whiskers indicate the maximum spread of the data, with outliers denoted as a red "+" sign. 


\section{Bibliography}

[1] J. H. M. van Delft, E. van Agen, S. G. J. van Breda, et al. Discrimination of genotoxic from non-genotoxic carcinogens by gene expression profiling. Carcinogenesis, 25(7):1265-1276, jan 2004.

[2] Alan Rodgman and Thomas Perfetti. The Chemical Components of Tobacco and Tobacco Smoke, Second Edition. CRC Press, feb 2013.

[3] Carl-Elis Boström, Per Gerde, Annika Hanberg, et al. Cancer risk assessment, indicators, and guidelines for polycyclic aromatic hydrocarbons in the ambient air. Environmental health perspectives, pages 451-88, jun 2002.

[4] Roger W L Godschalk, Frederik-Jan Van Schooten, and Helmut Bartsch. A critical evaluation of DNA adducts as biological markers for human exposure to polycyclic aromatic compounds. Journal of biochemistry and molecular biology, 36(1):1-11, jan 2003.

[5] Clinton Ifegwu, Kayode Osunjaye, Folasade Fashogbon, et al. Urinary 1-hydroxypyrene as a biomarker to carcinogenic polycyclic aromatic hydrocarbon exposure. Biomarkers in cancer, 4:7-17, 2012.

[6] K. Srogi. Monitoring of environmental exposure to polycyclic aromatic hydrocarbons: a review. Environmental Chemistry Letters, 5(4):169-195, oct 2007.

[7] Ki-Hyun Kim, Shamin Ara Jahan, Ehsanul Kabir, and Richard J.C. Brown. A review of airborne polycyclic aromatic hydrocarbons (PAHs) and their human health effects. Environment International, 60:71-80, oct 2013.

[8] Eshvendar Reddy Kasala, Lakshmi Narendra Bodduluru, Chandana C. Barua, Chandra Shekhar Sriram, and Ranadeep Gogoi. Benzo(a)pyrene induced lung cancer: Role of dietary phytochemicals in chemoprevention. Pharmacological Reports, 67(5):996-1009, 2015.

[9] Giorgio Vittorio Scagliotti, Purvish Parikh, Joachim von Pawel, et al. Phase III study comparing cisplatin plus gemcitabine with cisplatin plus pemetrexed in chemotherapy-naive patients with advanced-stage non-small-cell lung cancer. Journal of clinical oncology : official journal of the American Society of Clinical Oncology, 26(21):3543-51, jul 2008.

[10] Shaloam Dasari and Paul Bernard Tchounwou. Cisplatin in cancer therapy: molecular mechanisms of action. European journal of pharmacology, 740:364-78, oct 2014.

[11] Hamed Alborzinia, Suzan Can, Pavlo Holenya, et al. Real-Time Monitoring of Cisplatin-Induced Cell Death. PLoS ONE, 6(5):e19714, may 2011.

[12] Jae Sung Pyo, Hyun Kyoung Ju, Jeong Hill Park, and Sung Won Kwon. Determination of volatile biomarkers for apoptosis and necrosis by solid-phase microextraction-gas chromatography/mass spectrometry: A pharmacometabolomic approach to cisplatin's cytotoxicity to human lung cancer cell lines. Journal of Chromatography B, 876(2):170-174, dec 2008. 
[13] Johan van Meerloo, Gertjan J. L. Kaspers, and Jacqueline Cloos. Cell Sensitivity Assays: The MTT Assay. In Methods in molecular biology (Clifton, N.J.), volume 731, pages 237-245. 2011.

[14] A Baranska, A Smolinska, A W Boots, J W Dallinga, and F J van Schooten. Dynamic collection and analysis of volatile organic compounds from the headspace of cell cultures. Journal of Breath Research, 9(4):047102, oct 2015.

[15] A Smolinska, A-Ch C Hauschild, R R R Fijten, et al. Current breathomicsa review on data pre-processing techniques and machine learning in metabolomics breath analysis. Journal of breath research, 8(2):027105, jun 2014.

[16] G. E. P. Box and D. R. Cox. An analysis of transformations. Journal of the Royal Statistical Society. Series B (Methodological, pages 211-252, 1964.

[17] B. Walczak and D.L. Massart. Wavelets in Chemistry, volume 22. Elsevier Science B.V, nov 2000.

[18] Paul H. C. Eilers. A perfect smoother. Analytical Chemistry, 75(14):3631, jul 2003.

[19] Frank Dieterle, Alfred Ross, Götz Schlotterbeck, et al. Probabilistic quotient normalization as robust method to account for dilution of complex biological mixtures. Application in1H NMR metabonomics. Analytical Chemistry, 78(13):4281-4290, jul 2006.

[20] Leo Breiman. Random forests. Mach. Learn., 45(1):5-32, oct 2001.

[21] Ronny Schnabel, Rianne Fijten, Agnieszka Smolinska, et al. Analysis of volatile organic compounds in exhaled breath to diagnose ventilator-associated pneumonia. Scientific Reports, 5(October):17179, jan 2015 .

[22] Geou-Yarh Liou and Peter Storz. Reactive oxygen species in cancer. Free radical research, 44(5):479-96, may 2010.

[23] William M. Baird, Louisa A. Hooven, and Brinda Mahadevan. Carcinogenic polycyclic aromatic hydrocarbon-DNA adducts and mechanism of action. Environmental and Molecular Mutagenesis, 45(2-3):106-114, 2005.

[24] Q. Shi, L. Maas, C. Veith, F. J. Van Schooten, and R. W. Godschalk. Acidic cellular microenvironment modifies carcinogen-induced DNA damage and repair. Archives of Toxicology, 91(6):2425-2441, jun 2017.

[25] Q. Shi, A.W. Boots, L. Maas, et al. Effect of interleukin (IL)-8 on benzo[a]pyrene metabolism and DNA damage in human lung epithelial cells. Toxicology, 381:64-74, apr 2017.

[26] C M Kneepkens, Guy Lepage, Claude C. Roy, et al. The potential of the hydrocarbon breath test as a measure of lipid peroxidation. Free Radical Biology and Medicine, 17(2):127-60, aug 1994.

[27] Bogdan Calenic, Daniela Miricescu, Maria Greabu, et al. Oxidative stress and volatile organic compounds: interplay in pulmonary, cardio-vascular, digestive tract systems and cancer. Open Chemistry, 13(1), jan 2015.

[28] Meggie Hakim, Yoav Y. Broza, Orna Barash, et al. Volatile organic compounds of lung cancer and possible biochemical pathways. Chemical Reviews, 112(11):5949-5966, nov 2012.

[29] Cristian Palmiere, Frank Sporkert, Dominique Werner, et al. Blood, urine and vitreous isopropyl alcohol as biochemical markers in forensic investigations. Legal Medicine, 14(1):17-20, 2012.

[30] Bogusław Buszewski, Martyna Kȩsy, Tomasz Ligor, and Anton Amann. Human exhaled air analytics: biomarkers of diseases. Biomedical Chromatography, 21(6):553-566, jun 2007.

[31] Seul Ji Lee, Young Na Yum, Sang Cheol Kim, et al. Distinguishing between genotoxic and nongenotoxic hepatocarcinogens by gene expression profiling and bioinformatic pathway analysis. Scientific Reports, 3(1):2783, dec 2013.

[32] Reinskje Talhout, Thomas Schulz, Ewa Florek, et al. Hazardous compounds in tobacco smoke. International journal of environmental research and public health, 8(2):613-28, feb 2011.

[33] Hojae Shim and Shang-Tian Yang. Biodegradation of benzene, toluene, ethylbenzene, and oxylene by a coculture of Pseudomonas putida and Pseudomonas fluorescens immobilized in a fibrous-bed bioreactor. Journal of Biotechnology, 67(2-3):99-112, jan 1999. 
[34] V. Sedivec and J. Flek. The absorption, metabolism, and excretion of xylenes in man. International Archives of Occupational and Environmental Health, 37(3):205-217, sep 1976.

[35] A. Smolinska, A. G. L. Bodelier, J. W. Dallinga, et al. The potential of volatile organic compounds for the detection of active disease in patients with ulcerative colitis. Alimentary Pharmacology \&S Therapeutics, 45(9):1244-1254, may 2017.

[36] B de Lacy Costello, A Amann, H Al-Kateb, et al. A review of the volatiles from the healthy human body. Journal of breath research, 8(1):014001, mar 2014.

[37] David L. (David Lee) Nelson, Michael M. Cox, and Albert L. Lehninger. Lehninger principles of biochemistry. W.H. Freeman and Company, 2013.

[38] Zachary T. Schug, Johan Vande Voorde, and Eyal Gottlieb. The metabolic fate of acetate in cancer. Nature Reviews Cancer, 16(11):708-717, aug 2016.

[39] Dong Wang and Stephen J Lippard. Cisplatin-induced post-translational modification of histones H3 and H4. The Journal of biological chemistry, 279(20):20622-5, may 2004.

[40] Wojciech Filipiak, Andreas Sponring, Tomas Mikoviny, et al. Release of volatile organic compounds (VOCs) from the lung cancer cell line CALU-1 in vitro. Cancer Cell International, 8(1):17, nov 2008.

[41] Paweł Mochalski, Markus Theurl, Andreas Sponring, et al. Analysis of Volatile Organic Compounds Liberated and Metabolised by Human Umbilical Vein Endothelial Cells (HUVEC) In Vitro. Cell Biochemistry and Biophysics, 71(1):323-329, jan 2015.

[42] Agnieszka Baranska, Ettje Tigchelaar, Agnieszka Smolinska, et al. Profile of volatile organic compounds in exhaled breath changes as a result of gluten-free diet. Journal of breath research, 7(3):037104, sep 2013.

[43] Paweł Mochalski, Andreas Sponring, Julian King, et al. Release and uptake of volatile organic compounds by human hepatocellular carcinoma cells (HepG2) in vitro. Cancer Cell International, 13(1):72, jul 2013.

[44] Andreas Sponring, Wojciech Filipiak, Tomas Mikoviny, et al. Release of volatile organic compounds from the lung cancer cell line NCI-H2087 in vitro. Anticancer research, 29(1):419-26, jan 2009.

[45] Paweł Mochalski, Ramona Al-Zoairy, Andreas Niederwanger, Karl Unterkofler, and Anton Amann. Quantitative analysis of volatile organic compounds released and consumed by rat L6 skeletal muscle cells <i in vitro</i>. Journal of Breath Research, 8(4):046003, oct 2014.

[46] Luca Lavra, Alexandro Catini, Alessandra Ulivieri, et al. Investigation of VOCs associated with different characteristics of breast cancer cells. Scientific Reports, 5(1):13246, oct 2015.

[47] Hossam Haick, Lu Amal, Bin-bin Ding, et al. The scent fingerprint of hepatocarcinoma: invitro metastasis prediction with volatile organic compounds (VOCs). International Journal of Nanomedicine, 7:4135, jul 2012.

[48] Kristin Schallschmidt, Roland Becker, Christian Jung, et al. Investigation of cell culture volatilomes using solid phase micro extraction: Options and pitfalls exemplified with adenocarcinoma cell lines. Journal of Chromatography B, 1006:158-166, dec 2015.

[49] Tali Feinberg, Jens Herbig, Ingrid Kohl, et al. Cancer metabolism: the volatile signature of glycolysis < i > in vitro</i > model in lung cancer cells. Journal of Breath Research, 11(1):016008, jan 2017.

[50] Florence N Schleich, Jan W Dallinga, Monique Henket, et al. Volatile organic compounds discriminate between eosinophilic and neutrophilic inflammation in vitro. Journal of Breath Research, 10(1):016006, feb 2016.

[51] Nir Peled, Orna Barash, Ulrike Tisch, et al. Volatile fingerprints of cancer specific genetic mutations. Nanomedicine: Nanotechnology, Biology, and Medicine, 9(6):758-766, aug 2013.

[52] M. Sullivan, P. Galea, and S. Latif. What is the appropriate oxygen tension for in vitro culture? Molecular Human Reproduction, 12(11):653-653, sep 2006.

[53] Gregory J. Hannon. RNA interference. Nature, 418(6894):244-251, jul 2002.

[54] Jennifer A. Doudna and Emmanuelle Charpentier. The new frontier of genome engineering with CRISPR-Cas9. Science, 346(6213), 2014. 

CHAPTER 8

Determining the endogenous sources of Volatile Organic Compounds excreted after benzo[a]pyrene exposure in vitro

R Fijten, A Smolinska, Q Shi, D Pachen, J Dallinga, A Boots, F van Schooten

Submitted to PLOS comp. biol. 


\subsection{Abstract}

Breathomics is a new field of omics research that studies volatile organic compounds (VOCs) in exhaled breath to monitor or diagnose disease. However, the origin of these VOCs often remains elusive while understanding the underlying mechanisms behind these volatiles is important. To this end, we conducted an in vitro experiment that measured omics responses (transcriptomics, metabolomics and breathomics) in lung cells after exposure to benzo[a]pyrene. The aim of the study was to relate alterations in excreted VOC profiles to cellular metabolism and pathways. Lung cells were exposed to benzo[a]pyrene for $24 \mathrm{~h}$, after which the following biological extracts were harvested: RNA for microarray experiments and pathway analysis, intracellular metabolites (ICMs) for NMR spectroscopy and VOCs from headspace air for Gas Chromatography-Mass Spectrometry (GC-MS) analysis. Canonical Correlation Analysis (CCA) was performed to identify associations between VOCs, ICMs and pathways based on gene-set analysis in combination with multivariate statistics.

We found significant correlations between sets of VOCs and ICMs (coefficient = 0.97 p-value 0.0011) and between ICMs and four pathways databases (with a coefficient $>0.93$ and a p-value $<3.77 \mathrm{e}-04$ ). Spearman correlations between individual VOCs, ICMs and pathways, and subsequent network analysis resulted in potential endogenous sources for 5 of the 6 discriminatory VOCs, by connecting VOCs through metabolites to up- or down-regulated pathways.

In conclusion, this study demonstrated that chemical-induced alterations in cellular pathways are associated with excreted VOCs. This study might help to better understand the origin of various exhaled breath compounds and thus in the future redirect the focus of research to specific underlying pathways.

\subsection{Introduction}

Breathomics is a relatively new field of omics research that focuses on the use of exhaled breath to diagnose and monitor disease [1]. Exhaled breath consists of a mixture nitrogen, oxygen, carbon dioxide and noble gases [2]. In addition to these gases, trace amounts of volatile organic compounds (VOCs) can be detected in concentration in the nmol/l - pmol/l range $[1,2]$. The advantage of breathomics over other omics techniques in biofluids, such as blood and urine, is the non-invasive and easy collection method that simply requires normal breathing. Additionally, measurement and analysis of relevant VOCs can be performed quickly, resulting in a method with potential to diagnose diseases non-invasively and rapidly [3]. Exhaled breath analysis has been applied in a variety of diseases such as lung cancer [4-10], chronic obstructive pulmonary disease [11-14], asthma [15-17], breast cancer [18, 19], liver disease [20-24] and gastrointestinal disease [22, 25].

Even though thousands of VOCs are released into the exhaled breath, including hydrocarbons, aldehydes, ketones, alcohols, esters and aromatic hydrocarbons [8, 26], the origin of only a handful of VOCs has been identified. For instance, dimethylsulfide is exhaled as a result of fetor hepaticus [21, 27], while acetone is excreted via the lungs during ketoacidosis in diabetes [28]. 
For the majority of VOCs, only hypothetical endogenous sources have been proposed. For instance, hydrocarbons are produced as a result of oxidative stress and lipid peroxidation [29], whereas aldehydes are thought to originate from normal physiological processes such as signal transduction, proliferation and gene regulation [8]. Yet, many of these compounds can be produced by plants and foods or pollution [30]. Therefore, the validity of many VOCs as disease biomarkers is a subject of debate [30]. To limit the list of VOCs in the exhaled breath that are truly related to disease, the endogenous processes underlying the production of these volatile metabolites have to be investigated. In recent years, in vitro experiments that measure VOCs excreted by cells have emerged as a promising tool to investigate the endogenous sources of VOCs [31-33]. However, many of these studies simply compare VOCs that are produced by basal cellular metabolism [34-36], or compare cell lines with different characteristics [37-39]. Other studies have used a chemical trigger to investigate specific cellular processes [40, 41]. Even though these studies provide some insights in endogenous production of VOCs, direct links supported with evidence between VOCs and cellular processes have not yet been established.

Therefore, a more valuable approach would be to integrate in vitro breathomics with other omics technologies to gain insight in the systemic processes underlying the excreted VOCs [42].

The recent rise in high-throughput analysis of biological systems has enabled researchers to collect more than one dataset and combine them using omics integration methods [42]. The advantage of using multiple omics techniques is that it reflects the complexity of the biological system and thus provides additional insights, whereas utilization of one omics approach focuses one only one perspective of the studied problem [43]. Additionally, simultaneous analysis of multiple omics datasets enables researchers to look for causative changes that lead to disease throughout different information systems such as genes, proteins and metabolites [43]. Regarding breathomics, omics integration may provide new knowledge about the endogenous source of a VOC.

In this study, the pulmonary BEAS-2B cell line was exposed to benzo[a]pyrene $(\mathrm{B}[\mathrm{a}] \mathrm{P})$, after which transcriptomics, metabolomics and breathomics data was collected. Since the mechanism of action of B[a]P is well-understood [44], it provides the opportunity to combine this with previously acquired knowledge on $\mathrm{B}[\mathrm{a}] \mathrm{P}$ induced gene expression regulation, which is reflected by the collected transcriptomics data. Metabolomics analysis provides the link between the genetic regulation and the phenotype by directly measuring alterations in the cellular metabolism [45]. Since it is believed that VOCs are produced by the same metabolic processes as the metabolites, it is expected that associations between the excreted VOCs and metabolites can be identified. In this study, we aim to find the associations between VOCs, metabolites and pathways that will further result in a comprehensive multi-omics network that will give us insight in the endogenous sources of VOCs. This is the first study to use an omics integration approach to investigate the possible sources of endogenous VOCs excreted in vitro. Unravelling the origins is essential to expand our understanding of underlying processes and enhance new leads for disease monitoring. 


\subsection{Materials \& Methods}

\subsubsection{Cell culture and exposure}

BEAS-2B lung epithelial cells (ATCC CRL-9609) were cultured using Corning cell culture flasks (Corning Incorporated, New York, United States) in DMEMF-Dulbeccos Modified Eagles medium (DMEMF-12, Gibco, Invitrogen), supplemented with 1\% penicillin/streptomycin, $15 \mu / \mathrm{ml}$ bovine pituitary extract, $0.5 \mathrm{ng} / \mathrm{ml}$ bovine serum albumin (Invitrogen, Breda, the Netherlands), $10 \mathrm{ng} / \mathrm{ml}$ epidermal growth factor (Merck Millipore, Darmstadt, Germany), $10 \mathrm{ng} / \mathrm{ml}$ cholera-toxin (list Biological Laboratories, Inc., Campbell, California, USA), $5 \mu \mathrm{g} / \mathrm{ml}$ insulin, $5 \mu \mathrm{g} / \mathrm{ml}$ transferrin and $0.1 \mu \mathrm{M}$ dexamethasone (Sigma-Aldrich, St. Louis, MO, USA). The cells were cultured at $37 \mathrm{C}$ and $5 \% \mathrm{CO} 2$. The flasks with cells were randomly divided into two experimental groups: controls and exposed cells, each consisting of 16 samples. After reaching $80-90 \%$ confluency, the medium was replaced with fresh medium containing 1 $\mu \mathrm{M}$ benzo[a]pyrene (CAS no. 50-32-8; purity >96\%; Sigma-Aldrich, St. Louis, USA), to which the cells were exposed for 24 hours. The benzo[a]pyrene concentration was chosen to mimic non-lethal pollution-related exposures, and was determined by MTT cell proliferation assay [46].

\subsubsection{Collection of biological extracts}

Thirty-two samples were used to collect the following biological extracts: (i) VOCs excreted in the headspace of the cell culture flasks for breathomics, (ii) intracellular metabolites (ICMs) for metabolomics and (iii) RNA for transcriptomics.

\subsubsection{Headspace air for VOCs analysis by gas chromatography mass spectrometry}

The VOCs excreted by the epithelial cells were collected from the headspace above the cells for 24 hours as described previously [41]. Briefly, Corning flasks with cells were kept inside the incubator at $37 \mathrm{C}$ and connected to a purified mixture of N2 (75\%), O2 (20\%) and CO2 (5\%). A stainless steel two-bed sorption tube filled with carbograph 1TD/Carbopack X (Markes International, Llantrisant, Wales, UK) was connected to each flask to captured the emitted VOCs.

After collection of the VOCs from the headspace of 32 samples, the thermal desorption tubes were measured by gas chromatography time of flight mass spectrometry (GC-tof-MS) as previously described [41, 47]. Briefly, the thermal desorption tubes were heated to $350 \mathrm{C}$, releasing the captured VOCs. Seventy-five percent of the released VOCs was recaptured and stored back on the identical desorption tube, whereas $25 \%$ entered the gas chromatograph where the VOCs were separated. Time-of-flight mass spectrometry (tof-MS; Bench TOF-dx Almsco International, Llantrisant, Wales, $\mathrm{UK})$ ) was used to detect and identify the VOCs available in the samples. 


\subsubsection{Intracellular metabolites for nuclear magnetic resonance spec- troscopy}

After 24 hours of exposure, the cells were immediately washed with phosphate buffered saline and subsequently quenched with $3 \mathrm{ml}$ methanol at $-20^{\circ} \mathrm{C}$. The cells were detached from the flask with a cell scraper, after which $3 \mathrm{ml}$ chloroform $\left(-20^{\circ} \mathrm{C}\right)$ was added. Cell disruption was performed using a sonicator with a force of 15 micron in two cycles of 30s with a 30s pause [48]. After centrifuging for 5 minutes at $4^{\circ} \mathrm{C}$ (1600 RCF), three segments appeared: (i) polar phase including ICMs; (ii) interphase containing RNA and proteins, and (iii) non-polar phase with various molecules such as lipids. Each phase was stored in an individual $15 \mathrm{ml}$ CELLSTAR centrifuge tube at $-80{ }^{\circ} \mathrm{C}$ (VWR International, Radnor, Pennsylvania, USA) for further analysis. The polar phase containing the ICMs was first concentrated by overnight freeze-drying and stored immediately after at $-80{ }^{\circ} \mathrm{C}$ until Nuclear Magnetic Resonance (NMR) analysis. Right before NMR analysis, the metabolites were reconstituted by dissolving them in $292 \mu \mathrm{l}$ Milli-Q, $300 \mu \mathrm{l} \mathrm{D}$ O (99.9 atom \% D), $32 \mu \mathrm{l}$ of $1 \mathrm{M}$ dibasic sodium phosphate dehydrate buffer, $12 \mu \mathrm{l}$ of $5.5 \mathrm{mM}$ 4,4-dimethyl-4-silapentane-1-sulfonic acid (99.9 atom $\% \mathrm{D}$, for chemical shift reference; $\delta 0.00 \mathrm{ppm}$; final concentration: $0.1 \mathrm{mM}$ ) and $8 \mu \mathrm{l}$ of $0.5 \mathrm{M}$ sodium azide (with a final concentration of $6 \mathrm{mM}$ ). The samples were vortexed, centrifuged for $30 \mathrm{~s}$ at $4{ }^{\circ} \mathrm{C}(10.600 \mathrm{RCF})$ to remove any debris and pipetted into a clean $5 \mathrm{~mm} 600 \mathrm{MHz}$ NMR tube (Bruker, Billerica, MA, USA). 1H NMR spectra of all 32 samples were acquired on a Bruker Avance $700 \mathrm{MHz}$ system equipped with a TCI cryoProbe (Bruker, Billerica, MA, USA). 1D NOESY with water presaturation was used as solvent suppression [49]. Each spectrum contained 128 scans with a spectral width of $8418 \mathrm{~Hz}$ resulting in $32 \mathrm{~K}$ points. The acquisition time was set to 3.2 seconds, with 4 seconds of relaxation delay between scans. Subsequently, ACD/Spectrus Processor 2016.1.1 (Advanced Chemistry Development, Inc., Toronto, Canada) was used for spectral pre-treatment. All acquired free induction decays were zero-filled to $64 \mathrm{~K}$ data points, multiplied with a $0.3 \mathrm{~Hz}$ line-broadening function, Fourier transformed and manually phased.

\subsubsection{RNA for microarray experiments}

The extracted interphase contained RNA and proteins. Before RNA isolation, the interphase was washed with $2.5 \mathrm{ml}$ methanol at $-20^{\circ} \mathrm{C}$ and centrifuged for 10 minutes at $4^{\circ} \mathrm{C}(10.600 \mathrm{RCF})$. To remove the proteins from the interphase, the RNA was purified on columns using the miRNeasy Micro Kit (Qiagen, Venlo, the Netherlands). RNA quantities were determined using a Nano Drop ND-1000 spectrophotometer (Isogen, IJsselstein, the Netherlands) and the quality was assessed by RNA 6000 nanochips on the Agilent 2100 bioanalyzer (Agilent Technologies, Amsterdam, the Netherlands). An RNA Integrity Number (RIN) of $>8.0$ was considered suitable for further analysis, and 31 out of 32 samples had a RIN value of $>8.9$. That one samples was therefore excluded from further analyses. Labelling of total RNA (100 ng) was performed using an Affymetrix WT plus reagent kit and hybridized to whole genome Genechip Human Gene 1.1 ST arrays coding 19.697 genes (Affymetrix, Santa Clara, California, USA). Labelling, hybridization and image scanning of the samples were performed according to manufacturers instructions. 


\subsubsection{Data preprocessing}

\subsubsection{GC-MS chromatograms}

After the chromatograms were obtained, they were preprocessed as described previously by Smolinska et al. [50] to remove any non-biological variation. In short, the data was $\log$ transformed [51] and subsequently denoised by a Daubechies wavelet with two levels of compression [52]. Baseline correction and normalization were done by B-splines with asymmetric least squares smoothing (53) and probabilistic quotient normalization [53] respectively. Peak picking was carried out and peaks for the same compound were combined across sampled by correlation analysis of the mass spectra.

\subsubsection{NMR spectra}

The NMR spectral data were preprocessed, which typically involves baseline correction, alignment, binning and normalization. Baseline correction of NMR spectra was done by means of asymmetric least squares [54]. Sample temperature, $\mathrm{pH}$, ionic strength can cause fluctuation in the chemical shifts, therefore NMR spectra were aligned using correlation optimizing warping [55]. Adaptive intelligent binning was applied to reduce the high dimensionality of the NMR data (generally ca. 32,000 variables) [56]. The chemical shift ranges of $\delta 0.8 \quad 4.5$ and 4.98 .6 were used for binning procedure because they contained relevant information. To make the NMR spectral data comparable across all samples, the final step of data preprocessing consisted of normalization by probabilistic quotient normalization [53].

\subsubsection{Microarray data}

Quality control of the microarrays was performed on raw data by examining the signal distribution using principal component analysis (PCA) scatter plots, normal probability plots and MA-plots [57]. All 31 arrays were of sufficient quality for further analysis. After the quality control, custom annotation was performed based on reorganized oligonucleotide probes [58] and expression values were normalized by the robust multichip average method [59]. After annotation and normalization, the genes were grouped into sets that are based on genes present in validated pathways from the four most comprehensive biological databases: (i) BioCarta [60]: 217 pathway genesets available from MSigDb [61]; (ii) KEGG [43]: 186 pathway genesets available from MSigDb [61]; (iii) Reactome [62, 63]: 674 pathway genesets available from MSigDb [61] and (iv) WikiPathways [64, 65]: 380 pathway genesets available from WikiPathways.

For each set, the expression values of its genes were combined to create a pathway expression value using the Pathway Level Analysis of Gene Expression method [66]. These pathway expression values were used in the subsequent data analysis. 


\subsubsection{Data analysis}

\subsubsection{Random Forests and correlation analysis}

The statistical analysis employed here consisted of two steps. The first step was designed to select the most discriminatory VOCs, metabolites and pathways between control cells and cells exposed to B[a]P. The second step aimed to find the relationship between the discriminatory sets of variables found in the first phase. A selection of discriminatory variables (VOCs, ICMs and pathways) was made using Random Forests (RF) [67]. In each case, a model was created to separate the controls from the exposed cells and was internally validated by means of the out-of-bag error. The algorithm also calculates the importance of a specific variable to distinguish exposed and non-exposed cells. The RF analysis resulted in sets of discriminatory variables for the VOCs, ICMs and pathways. In order to assess the relationship between discriminatory VOCs, ICMs and pathways, canonical correlation analysis (CCA) is used in combination with PCA [68]. The main aim of the CCA technique is to find the relationship between two multivariate sets of variables measured for the same set of samples. CCA is considered an extension of bivariate correlations. Since CCA is only applicable when more samples than parameters are available, PCA was first employed for dimension reduction. The CCA analysis was performed for the following comparisons: (i) VOCs vs. ICMs; (ii) VOCs vs. BioCarta pathways; (iii) VOCs vs. KEGG pathways; (iv) VOCs vs. Reactome pathways; (v) VOCs vs. WikiPathways; (vi) ICMs vs. BioCarta pathways; (vii) ICMs vs. KEGG pathways; (viii) ICMs vs. Reactome pathways; (ix) ICMs vs. WikiPathways.

In order to show the pair-wise relationships between the individual VOCs, ICMs and pathways, a spearman pair-wise correlation was calculated between the most contributing sets. The correlations were considered significant if the corresponding p-value was less than 0.05 .

\subsubsection{Chemical identification of VOCs and intracellular metabolites}

The putative identification of the discriminatory VOCs was done by examination of their retention time and mass spectrum using the National Institute of Standard and Technology library in conjunction with an in-house library with pure compounds. As a final validation, an experienced mass spectrometrist evaluated and confirmed the identities of the VOCs. The discriminatory intracellular metabolites were identified using the $600 \mathrm{MHz}$ library of metabolite NMR spectra from the Chenomx NMR Suite $8.3(\mathrm{pH} 6-8)$ [70].

\subsection{Results}

VOCs in the headspace and ICMs were collected and analyzed from 32 cell culture flasks (16 controls and 16 exposed to $\mathrm{B}[\mathrm{a}] \mathrm{P})$. Microarray analysis was performed in 31 cell culture flasks (15 controls and 16 exposed to $\mathrm{B}[\mathrm{a}] \mathrm{P})$. The data preprocessing of breathomics, metabolomics and transcriptomics data resulted in 191 VOCs, 517 NMR bins and 1457 pathways. The pathways were subdivided into 217 BioCarta, 
186 KEGG, 674 Reactome and 380 WikiPathways pathways. The first part of the statistical analysis consisted of RF modeling with the aim to select discriminatory VOCs, ICMs and pathways between controls and exposed cells. This resulted in the selection of 6 VOCs and 17 NMR bins corresponding to 5 ICMs. For the pathways, 10 BioCarta, 9 KEGG, 33 Reactome and 19 WikiPathways pathways were chosen. The corresponding chemical identities of the VOCs and metabolites, and the full name of the pathways can be found in the supplementary tables 8.S1-8.S6.

\subsubsection{Canonical Correlation Analysis}

The CCA analysis was performed as a second step in the statistical analysis to investigate the correlation between the 6 discriminatory VOCs, 5 discriminatory ICMs and the four groups of pathways. CCA analysis between the VOCs from the headspace and the four groups of pathways did not reveal any statistically significant correlations.

\subsubsection{VOCs vs. ICMs}

CCA analysis between the VOCs from headspace and the ICMs showed a statistically significant correlation of 0.97 (p-value 0.0011). The corresponding CCA correlation plot is shown in Figure 8.1.

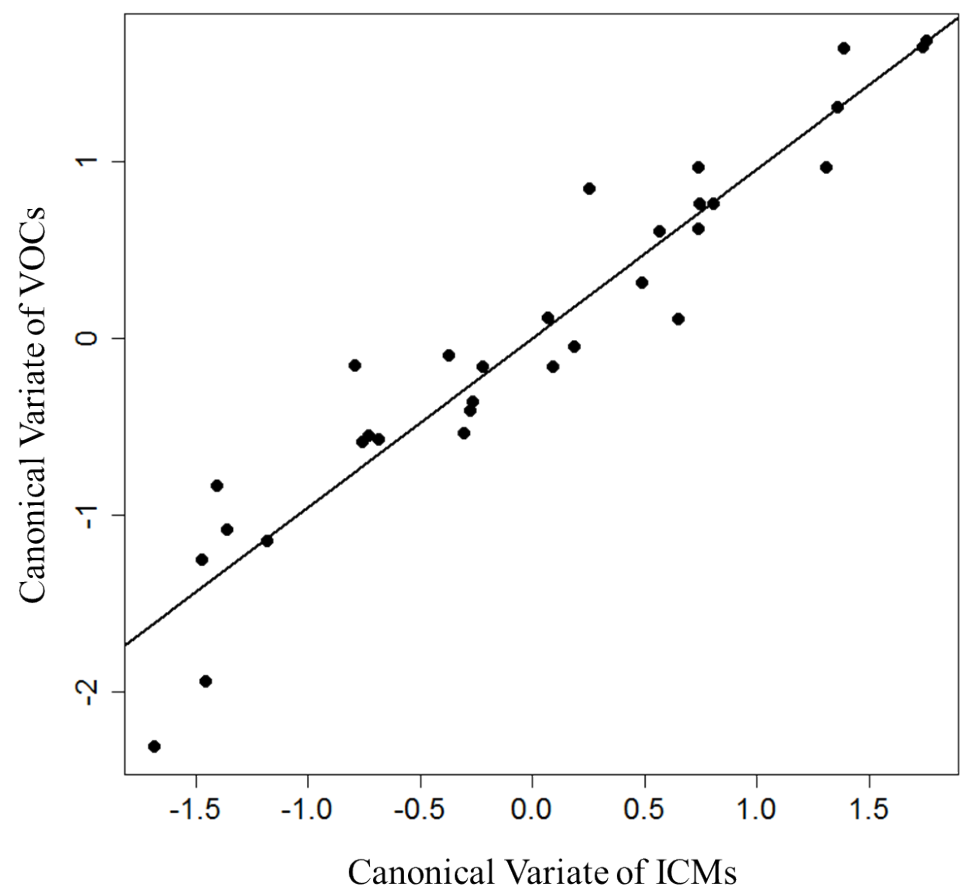

Figure 8.1: The canonical correlation score plot using the first canonical variates of selected sets of the most important ICMs (x-axis) and VOCs (y-axis). 


\subsubsection{Intracellular metabolites vs. Pathways}

The four separate pathway datasets were correlated by CCA to the ICMs. The CCA analysis performed on the comparison between the ICMs and BioCarta pathways yielded a correlation coefficient of 0.93 ( $\mathrm{p}$-value $=1.22 \mathrm{e}-05$; figure $8.2(\mathrm{a})$ ). The CCA analysis between ICMs and KEGG pathways produced a coefficient of 0.98 (p-value $=3.77 \mathrm{e}-04$; figure 8.2(b)). When correlating the ICMs with the Reactome pathways, a correlation coefficient of 0.98 (p-value $=9.09 \mathrm{e}-06$; figure $8.2(\mathrm{c})$ ) was achieved. The fourth CCA analysis was performed on the comparison between the ICMs and WikiPathways and resulted in a correlation coefficient of 0.98 ( $\mathrm{p}$-value $=5.03 \mathrm{e}-07$; figure $8.2(\mathrm{~d})$ ).
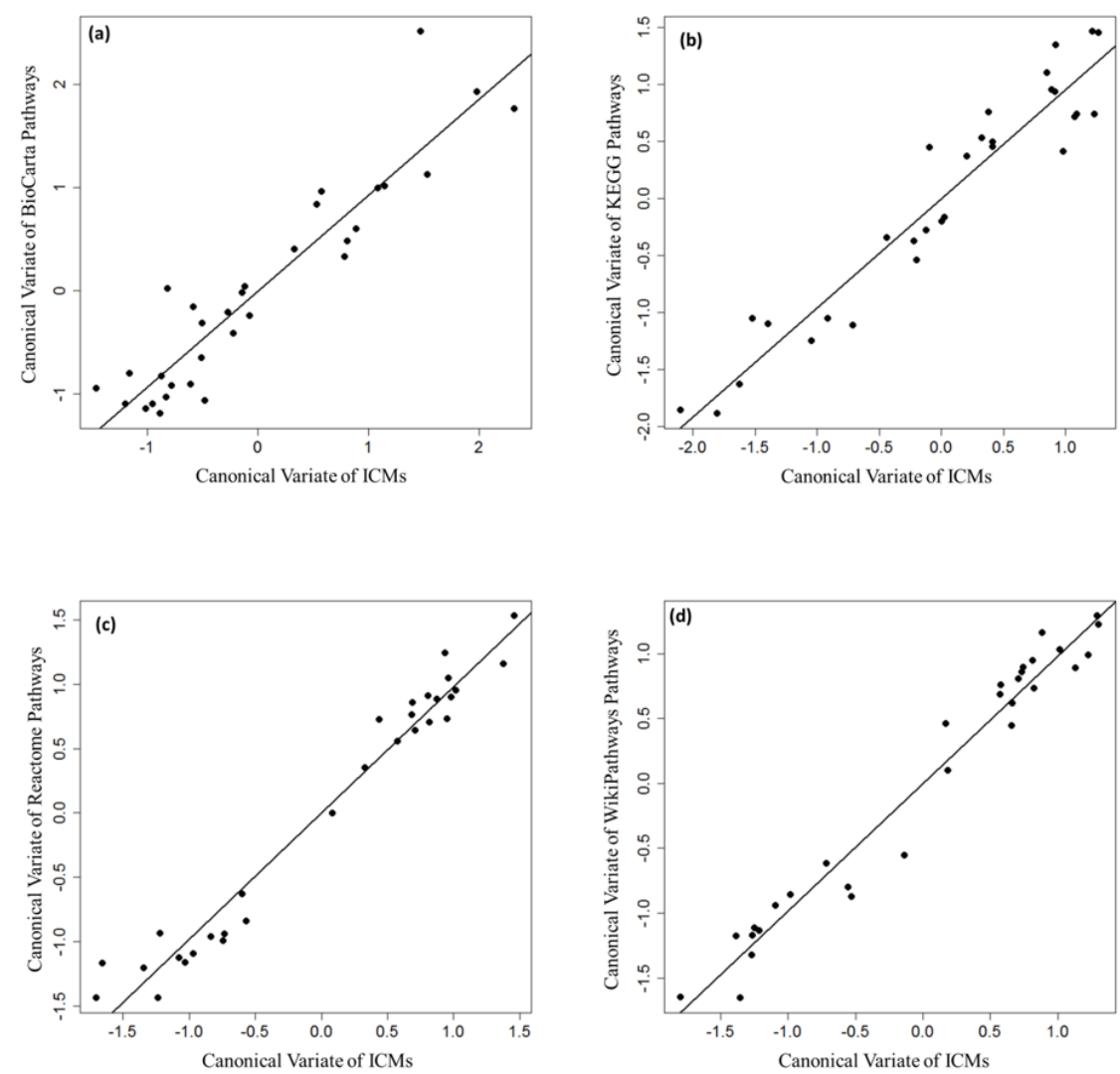

Figure 8.2: Canonical correlation analysis results of the comparisons between the ICMs and the pathways. (a) ICMs and BioCarta pathways. (b) ICMs and KEGG pathways. (c) ICMs and Reactome pathways. (d) ICMS and WikiPathways. 


\subsubsection{Pair-wise correlations}

Since CCA revealed significant correlations between the sets of VOCs and ICMs, and the sets of ICMs and pathways, pair-wise Spearman correlations were calculated for the following comparisons: (i) VOCs vs. ICMs, (ii) ICMs vs. BioCarta pathways, (iii) ICMs vs. KEGG pathways, (iv) ICMs vs. Reactome pathways and (v) ICMs vs. WikiPathways.

\subsubsection{VOCs vs. intracellular metabolites}

Pair-wise correlations were calculated between the 6 discriminatory VOCs and the 5 discriminatory ICMs and are visualized in the heatmap in figure 8.3 where significant correlations are indicated with an asterisk. The figure demonstrates that most pairwise correlations were positive and not significant. However, it is notable that the strongest and most significant correlations are negative ones. Nonanal and decanal, both aldehydes, reveal similar correlation patterns with the ICMs, showing strong negative correlations with myo-inositol and to a lesser extent with dimethylamine. Interestingly, one of the three bins representing myo-inositol displays a different correlation pattern than the other two. Additionally, one glutamine bin is significantly negatively correlated to octadecane, but every other bin is positively correlated to the VOC levels. These findings might be explained by slight overlap with another ICMs in NMR spectrum.

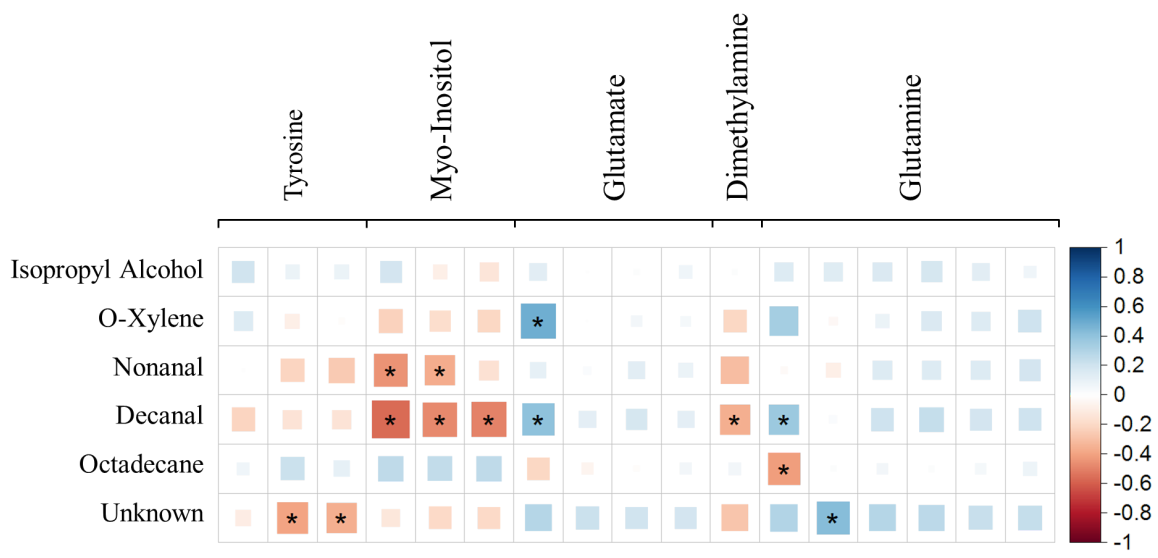

Figure 8.3: Heat map of the pair-wise correlations between the discriminatory ICMs (x-axis) and the discriminatory VOCs (y-axis). The x-axis consists of NMR bins that belong to a certain metabolite. The size of a square and the intensity of the color signify the degree of correlation as displayed by the color bar. An asterisk indicates a significance of $p<0.05$. 


\subsubsection{Intracellular metabolites vs. Pathways}

The 5 discriminatory ICMs were used for pair-wise correlations with four sets of pathways. A subset of 10 BioCarta, 9 KEGG, 33 Reactome and 19 WikiPathways were chosen for each pathway database. The correlations are displayed in figure 8.4(a)-(d) and display a mixture of positive and negative correlations of which the majority is significant. Remarkably, the correlations observed between ICMs and Reactome and WikiPathways (Figures 8.4(c) and (d)) revealed only few non-significant relations as designated by a hashtag. However, the correlations with BioCarta and KEGG pathways were not as pronounced as the ones with the Reactome pathways and WikiPathways. The BioCarta and KEGG heatmaps display a predominant negative correlation of most pathways with myo-inositol. Correlations with other metabolites demonstrated opposite behavior when comparing the two pathway databases, such as glutamine and glutamate, which are positively and significantly correlated with most BioCarta pathways and negatively correlated with most KEGG pathways.

Interestingly, in the Reactome and WikiPathways heatmaps, two clusters of correlations appear, each representing a different subset of pathways. For instance, the lower Reactome cluster (cluster 2, figure 8.4c) contains DNA repair pathways and is positively correlated with tyrosine and myo-inositol. However, the opposite is observed for the WikiPathways heatmap (figure 8.4d), where the DNA damage response pathways are in cluster (cluster 1) and are negatively correlated with tyrosine and myo-inositol.

Correlation network between VOCs, ICMs and pathways A correlation network was created to integrate all VOCs, ICMs and pathways that revealed significant pairwise Spearman correlations shown previously in figure 8.4. The network is shown in figure 8.5 and exhibits the complex relationships between VOCs, ICMs and pathways. The three different shapes of nodes represent the three omics datasets, with a circle for the pathways (with a different color for each database), a green diamond for the ICMs and a red square for the VOCs. The pathways in the clusters in the Reactome and WikiPathways heatmaps were combined for clarity as they displayed the same information (clusters 1 and 2 in figure 8.4(c) and (d), respectively). The network holds 130 different significant correlations (indicated as a solid line), which are colored based on the correlation coefficients, ranging from red for negative correlations to blue for positive correlations. The network shows that WikiPathways cluster 2 is strongly negatively correlated with glutamate, which in turn is positively correlated with Oxylene. Additionally, the BioCarta pathways are positively correlated with glutamate and glutamine, and negatively correlated with tyrosine and myo-inositol, which are in turn negatively correlated with decanal, nonanal and the unknown VOC. The figure also clearly demonstrates that the two clusters of the Reactome and WikiPathways pathways behave in the opposite way from the other, whereas the pathways from BioCarta and KEGG generally behave in a similar manner for a specific ICM.

\subsection{Discussion \& Conclusion}

The discovery of the endogenous sources of VOCs is a major challenges in exhaled breath research, yet is difficult [30]. In vitro studies may shed light on the endoge- 


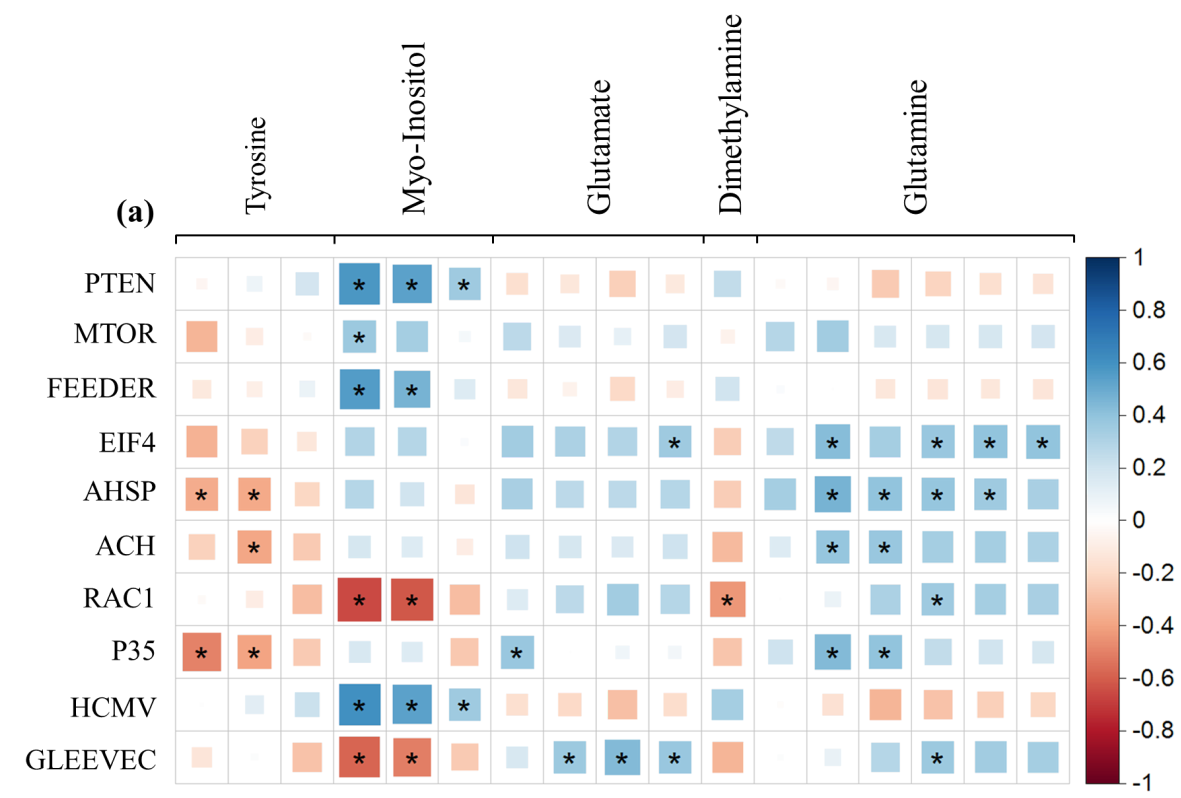

Figure 8.4: Heat maps of individual correlations between ICMs (x-axis) and discriminatory pathways from the following four databases: (a) BioCarta, (b) KEGG, (c) Reactome and (d) WikiPathways. The x-axis displays the NMR bins corresponding to a specific metabolite. The names on the y-axes are shortened names of the discriminatory pathways. An asterisk indicates a significance of $\mathrm{p}<0.05$ in (a) and (b), whereas a \# indicates a non-significant correlation in (c) and $(\mathrm{d})$.

nous source of VOCs. Several approaches to measure VOCs in headspace in vitro can be used, ranging from measuring basal cellular metabolism to using chemical triggers to elicit a well-defined response from cells. However, the most informative and optimal approach to determine the endogenous source of a specific VOC is to link headspace VOCs to other omics datasets like transcriptomics and metabolomics from the same experiment. Therefore, the aim of this study was to gain more insight in the endogenous sources of VOCs by integration of breathomics, metabolomics and transcriptomics data. We investigated this aim by exposing an in vitro pulmonary cell line to $\mathrm{B}[\mathrm{a}] \mathrm{P}$ to induce a well-understood cellular response. After exposure, the three types of omics were collected and analyzed. Subsets of VOCs, ICMs and pathways were selected based on the distinction between exposed and non-exposed cells. These were then integrated by CCA to determine whether significant associations existed between these subsets, and subsequent pair-wise Spearman correlations to find relations between individual VOCs, ICMs and pathways. This study is the first to use multiple omics integration to determine the plausible endogenous sources of VOCs. We provide several presumed endogenous sources of VOCs by linking them to specific pathways. We found 6 VOCs excreted by cells that were related to the changes made 
(b)

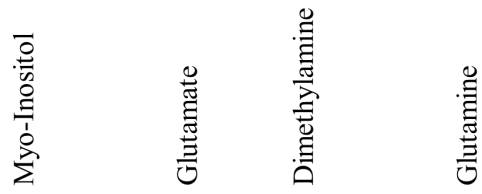

RNA Polymerase

Autophagy

*

Glycox. \& Dicarbox. Met.

Taste Transduction

Parkinson's

* $\square$

$*$ 


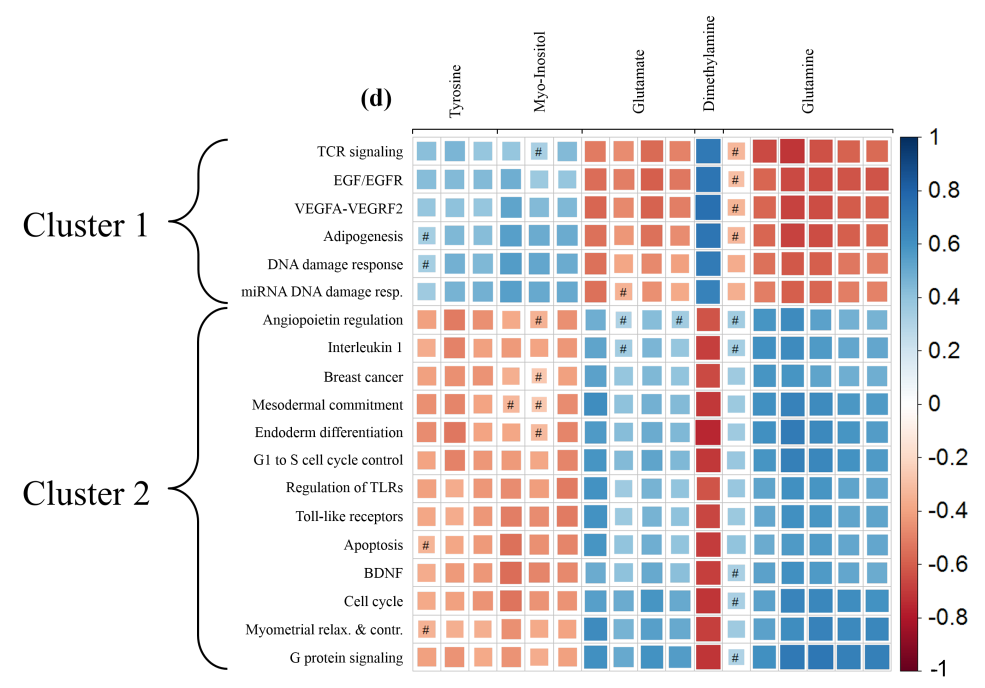

Figure 8.4: (Continued.)

by the exposure to B[a]P. Five of the six discriminatory VOCs revealed one or more significant pair-wise correlation with one or more metabolite, which in turn was significantly associated to a large number of biological gene expression pathways. The suggestions for possible explanation of the endogenous origin of these five VOCs are described below together with the evidence obtained from scientific literature.

\subsubsection{Octadecane}

Octadecane is an alkane of which the concentration was higher after $\mathrm{B}[\mathrm{a}] \mathrm{P}$ exposure. Researchers have hypothesized that alkanes such as octadecane are produced as a result of oxidative stress and lipid peroxidation [8]. In this study, it was negatively correlated with glutamine, an amino acid that is present in the cell culture medium. Glutamine is involved in the biosynthesis of proteins and lipids, and is a frequent source of energy and carbon for the cells. It was present in the cells in a lower concentration after exposure, suggesting a reduced uptake from the cell culture medium due to DNA damage that occurred after the exposure [71]. Glutamine is negatively correlated with WikiPathways cluster 1 that contains two upregulated pathways related to DNA damage response, confirming the link between reduced glutamine uptake and DNA damage. Glutamine was also positively correlated with all discriminatory BioCarta pathways, of which most contain parts of the Akt/Pi3K pathway. The Akt/Pi3K pathways is an intracellular signaling pathway that regulates the cell cycle and is related to apoptosis and proliferation [72]. The positive correlation between glutamine and the BioCarta pathways contradicts other research that has shown that glutamine deprivation can upregulate the Pi3K/Akt pathway [73] and induce apoptosis [74], suggesting a negative correlation. It is possible that this discrepancy is a result of a delay in pathway expression as opposed to metabolite levels. Moreover, a 


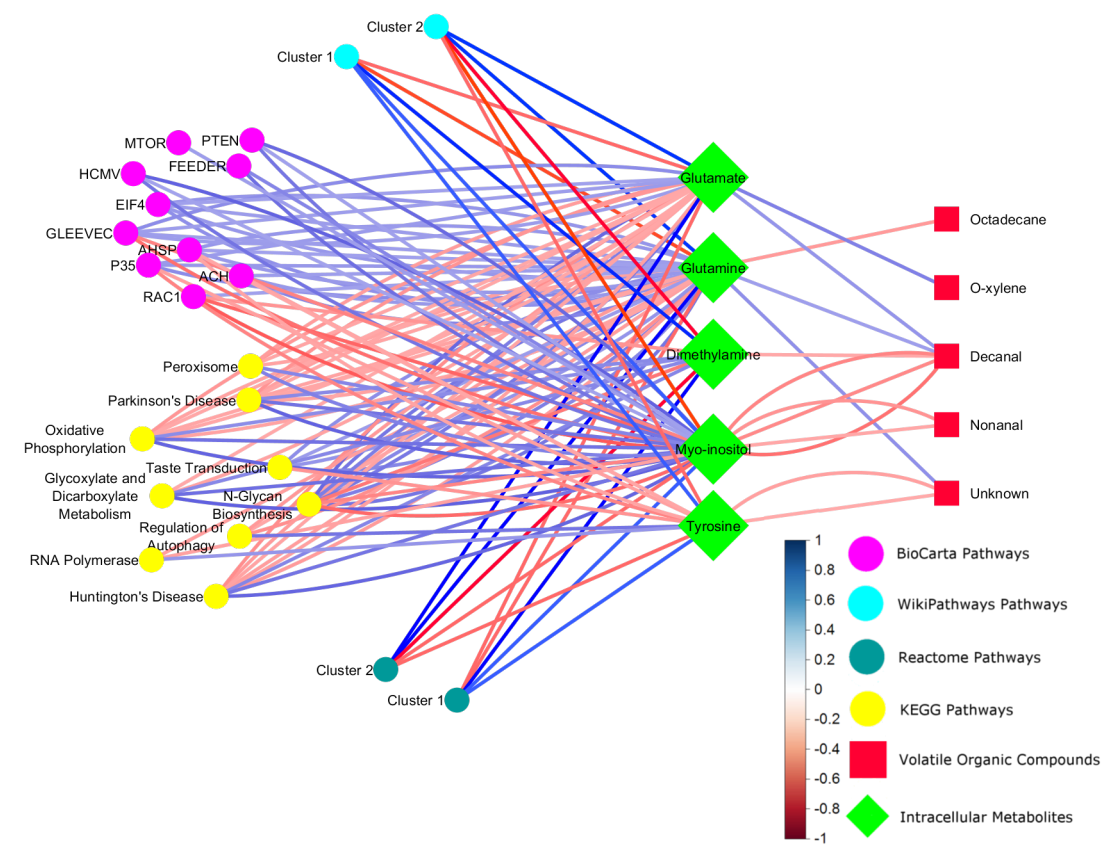

Figure 8.5: Network of the significant pair-wise Spearman correlations ( $\mathbf{p}<\mathbf{0 . 0 5})$ between all three omics datasets. The nodes represent pathways, metabolites and VOCs, whereas the lines demonstrate the significant correlations. The color of a line indicates the correlation coefficient between the two nodes it connects as indicated in the color legend on the bottom right corner.

positive correlation was found between glutamine and the KEGG pathway N-Glycan Biosynthesis. Since glutamine is needed for the first step of n-glycan biosynthesis [75], reduced glutamine uptake will lead to a reduced biosynthesis of n-glycans. The fact that octadecane was negatively correlated with glutamine in this study suggests that it is associated with increased DNA damage and apoptosis, and reduced biosynthesis of cellular building blocks such as N-Glycans. Although oxidative stress pathways were not found as discriminatory in this study, B[a]P does induce oxidative stress and subsequent lipid peroxidation due to the induction of DNA damage [76]. The hypothesized link between alkanes and oxidative stress is therefore substantiated based on these results. 


\subsubsection{O-xylene}

The excreted concentration of the cyclic VOC o-xylene was downregulated after B[a]P exposure. It has been found previously in feces, breath, breast milk, blood and saliva (26), and in two in vitro studies [37,39]. Lavra et al. found increased levels of o-xylene in cancer cell lines compared to non-transformed cell lines [37]. Schallschmidt et al. found decreased concentrations of o-xylene in the A549 lung cancer cell line compared to medium [39]. However, these findings are conflicting with the notion that o-xylene is considered exogenous [77]. It is therefore unclear what the exact source of exhaled or in vitro excreted VOCs is.

It was positively correlated with glutamate, which concentration was also downregulated in exposed cells. Glutamate is a precursor for glutamine [78] and acts in a similar manner as glutamine in the correlations to the pathways. This evidence suggests that processes related to glutamine, such as DNA damage response and Akt/Pi3K, are also related to glutamate. However, no direct link exists between glutamate and the Akt/Pi3K pathway or DNA damage in tissues other than the brain [79]. Still, these results suggest that o-xylene may be used as a marker of the DNA damage response as a result of exposure to carcinogens like $\mathrm{B}[\mathrm{a}] \mathrm{P}$. However, since o-xylene is considered exogenous, this hypothesis needs to be confirmed by future research.

\subsubsection{Decanal and nonanal}

Decanal and nonanal are aldehydes that were downregulated after exposure to B[a]P. These aldehydes are produced as a result of normal physiological processes such as signal transduction, proliferation and gene regulation [8], which may be disrupted by the $\mathrm{B}[\mathrm{a}] \mathrm{P}$ exposure. Decanal was positively correlated with glutamate and glutamine and negatively correlated to dimethylamine and myo-inositol, whereas nonanal was only negatively correlated with myo-inositol. As stated above, glutamine is involved in several processes, mainly the biosynthesis of proteins and lipids, and is associated with DNA damage responses and Akt/Pi3K. Glutamate is a precursor for glutamine [78], suggesting that the same processes can be linked to both. Dimethylamine is a degradation byproduct of assymetric dimethylarginine (ADMA) [80], which in turn is produced as a result of protein degradation [81]. No evidence exists that B[a]P directly induces proteolysis, but proteolysis has been linked to apoptosis [82]. In terms of pathways, dimethylamine is positively correlated with for instance the WikiPathway DNA damage response and negatively correlated with most of the pathways in WikiPathways cluster 2 , which includes pathways related to the cell cycle and normal biological processes. The latter metabolite, myo-inositol, is a glucose isomer [83] and is involved in a multitude of processes such as cell signaling and proliferation. Interestingly, it can inhibit the Akt/Pi3K pathway [83], which is represented in most BioCarta pathways. For example, the BioCarta Gleevec pathway describes cell proliferation and is negatively correlated with myo-inositol. Since Akt promotes cell proliferation [84], the data suggests that the level of myo-inositol is increased to combat over-proliferation. Another BioCarta pathway, PTEN, was positively correlated with myo-inositol levels, which means it was also upregulated after exposure. This pathway mainly revolves around apoptosis and cell cycle arrest; the opposite of the Gleevec pathway. These results support the same conclusion that level of myo-inositol is upregulated as a com- 
pensation mechanism for over-proliferation and potential tumor formation. Decanal and nonanal are therefore potential markers of proliferation and apoptosis, which is in concordance with the hypothesis that they are produced by proliferation, signal transduction and gene regulation [8].

\subsubsection{Unknown VOC}

Finally, the unknown VOC was found in a lower concentration after exposure to $\mathrm{B}[\mathrm{a}] \mathrm{P}$ and was positively correlated with glutamine and negatively correlated with tyrosine. Since glutamine-uptake is reduced after B[a]P exposure, the unknown VOC may be related to DNA damage similarly to octadecane. Additionally, it was negatively correlated to tyrosine, which was heightened after exposure. Tyrosine is a non-essential amino acid that is involved in a multitude of signal transduction pathways [84]. In addition to being present in the cell culture medium, it is synthesized by mammals from the amino acid phenylalanine [84], which is also present in the cell culture medium. Tyrosine is positively correlated with WikiPathways cluster 1, which contains a variety of signaling pathways. This indicates that upon $\mathrm{B}[\mathrm{a}] \mathrm{P}$ exposure, signal transduction pathways are upregulated, resulting in a larger uptake of tyrosine from the medium and potential upregulation of tyrosine biosynthesis from phenylalanine to supply these upregulated pathways. These findings suggest that the unknown VOC may be indicative of alterations in signal transduction.

\subsubsection{Conclusion}

Summarizing, five of the six discriminatory VOCs had significant correlations with one or more metabolites. These results in combination with established literature suggest that the alkane octadecane is a likely biomarker of oxidative stress. O-xylene may be linked to DNA damage responses, nevertheless it is considered exogenous, so the evidence for this link needs further investigation. Moreover, the results obtained for decanal and nonanal support the scientific evidence that the aldehydes are potential markers of cell proliferation and apoptosis. Finally, the unknown VOC is related to tyrosine, which suggests a link with signal transduction. However, without a chemical identity of this VOC, the current findings are only speculations.

In conclusion, several possible insights on alleged endogenous sources of VOCs have been identified, yet it is important to note that association does not equal causation. It is, therefore, essential that these findings are validated in in vitro models by sampling headspace VOCs after for instance manipulation of the above identified pathways.

\subsection{Supplementary Materials}


Table 8.S1: The discriminatory VOCs, their CAS numbers and information about whether they were present in higher or lower concentrations after exposure.

\begin{tabular}{lll}
\hline VOC name & CAS number & Up/down in exposed \\
\hline Isopropyl Alcohol & $67-63-0$ & Up \\
O-Xylene & $95-47-6$ & Down \\
Nonanal & $124-19-6$ & Down \\
Decanal & $112-31-2$ & Down \\
Octadecane & $593-45-3$ & Up \\
Unknown & NA & Down
\end{tabular}

Table 8.S2: The discriminatory intracellular metabolites and their respective CAS numbers. Additionally, information was included about whether they were present in higher or lower concentrations after exposure.

\begin{tabular}{lll}
\hline Metabolite name & CAS number & Up/down in exposed \\
\hline Tyrosine & $60-18-4$ & $\mathrm{Up}$ \\
Myo-inositol & $87-89-8$ & $\mathrm{Up}$ \\
Glutamate & $56-86-0$ & Down \\
Dimethylamine & $124-40-3$ & $\mathrm{Up}$ \\
Glutamine & $56-85-9$ & Down
\end{tabular}

Table 8.S3: Full names of the BioCarta pathways. The up- or downregulation of each pathway after exposure compared to controls is included.

\begin{tabular}{ll}
\hline pathway name & $\begin{array}{l}\text { Up/down in } \\
\text { exposed }\end{array}$ \\
\hline PTEN PATHWAY & Up \\
MTOR PATHWAY & Down \\
FEEDER PATHWAY & Up \\
EIF4 PATHWAY & Down \\
AHSP PATHWAY & Down \\
ACH PATHWAY & Down \\
RAC1 PATHWAY & Down \\
P35ALZHEIMERS PATHWAY & Down \\
HCMV PATHWAY & Up \\
GLEEVEC PATHWAY & Down
\end{tabular}


Table 8.S4: Full names of the KEGG pathways. The up- or downregulation of each pathway after exposure compared to controls is included.

\begin{tabular}{ll}
\hline pathway name & $\begin{array}{l}\text { Up/down in } \\
\text { exposed }\end{array}$ \\
\hline Rna Polymerase & $\mathrm{Up}$ \\
Regulation Of Autophagy & $\mathrm{Up}$ \\
Glyoxylate And Dicarboxylate Metabolism & $\mathrm{Up}$ \\
Taste Transduction & Down \\
Parkinsons Disease & $\mathrm{Up}$ \\
Oxidative Phosphorylation & $\mathrm{Up}$ \\
Peroxisome & $\mathrm{Up}$ \\
N Glycan Biosynthesis & Down \\
Huntingtons Disease & $\mathrm{Up}$
\end{tabular}


Table 8.S5: Full names of the Reactome pathways. The up- or downregulation of each pathway after exposure compared to controls is included.

\begin{tabular}{|c|c|}
\hline pathway name & $\begin{array}{l}\text { Up/down in } \\
\text { exposed }\end{array}$ \\
\hline Ngf Signalling Via Trka From The Plasma Membrane & Up \\
\hline Immune System & Up \\
\hline Signaling By Fgfr In Disease & Up \\
\hline Signalling By Ngf & Up \\
\hline Signaling By Erbb2 & Up \\
\hline Signaling By Fgfr & Up \\
\hline Diabetes Pathways & Up \\
\hline Downstream Signaling Of Activated Fgfr & Up \\
\hline Metabolism Of Carbohydrates & Down \\
\hline Nucleotide Excision Repair & Down \\
\hline Activated Tlr4 Signalling & Up \\
\hline Signaling By Egfr In Cancer & Up \\
\hline Cytokine Signaling In Immune System & Up \\
\hline Global Genomic Ner Gg Ner & Down \\
\hline Toll Receptor Cascades & Up \\
\hline $\begin{array}{l}\text { Neurotransmitter Receptor Binding And Down- } \\
\text { stream Transmission In The Postsynaptic cell }\end{array}$ & Up \\
\hline Myd88 Mal Cascade Initiated On Plasma Membrane & Down \\
\hline Developmental Biology & Up \\
\hline Trif Mediated Tlr3 Signaling & Down \\
\hline Phospholipid Metabolism & Down \\
\hline Platelet Activation Signaling And Aggregation & Up \\
\hline Innate Immune System & Down \\
\hline Rna Pol Ii Transcription & Up \\
\hline $\begin{array}{l}\text { Traf6 Mediated Induction Of Nfkb And Map Kinases } \\
\text { Upon Tlr7 } 8 \text { Or } 9 \text { Activation }\end{array}$ & Down \\
\hline Transmission Across Chemical Synapses & Up \\
\hline Metabolism Of Lipids And Lipoproteins & Up \\
\hline Cell Surface Interactions At The Vascular Wall & Up \\
\hline Generic Transcription Pathway & Up \\
\hline Dna Repair & Down \\
\hline $\begin{array}{l}\text { Nfkb And Map Kinases Activation Mediated By Tlr4 } \\
\text { Signaling Repertoire }\end{array}$ & Down \\
\hline Signaling By Scf Kit & Up \\
\hline Trans Golgi Network Vesicle Budding & Down \\
\hline Pol Switching & Down \\
\hline
\end{tabular}


Table 8.S6: Full names of the WikiPathways. The up- or downregulation of each pathway after exposure compared to controls is included.

\begin{tabular}{ll}
\hline Pathway Name & $\begin{array}{l}\text { Up/down in } \\
\text { exposed }\end{array}$ \\
\hline VEGFA-VEGFR2 Signaling Pathway & Down \\
DNA Damage Response & $\mathrm{Up}$ \\
T-Cell antigen Receptor (TCR) Signaling Pathway & $\mathrm{Up}$ \\
miRNA Regulation of DNA Damage Response & $\mathrm{Up}$ \\
Integrated Breast Cancer Pathway & $\mathrm{Up}$ \\
Mesodermal Commitment Pathway & Down \\
Endoderm Differentiation & Down \\
EGF/EGFR Signaling Pathway & Down \\
Structural Pathway of Interleukin 1 (IL-1) & Up \\
Adipogenesis & Down \\
Angiopoietin Like Protein 8 Regulatory Pathway & Up \\
Toll-like Receptor Signaling Pathway & Down \\
Myometrial Relaxation and Contraction Pathways & Down \\
G1 to S cell cycle control & Down \\
G Protein Signaling Pathways & Down \\
Regulation of toll-like receptor signaling pathway & Down \\
Brain-Derived Neurotrophic Factor (BDNF) signal- & Down \\
ing pathway & \\
Cell Cycle & Down
\end{tabular}


Chapter 8. Determining endogenous sources of VOCs 


\section{Bibliography}

[1] Agnes W Boots, Joep J B N van Berkel, Jan W Dallinga, et al. The versatile use of exhaled volatile organic compounds in human health and disease. Journal of Breath Research, 6(2):27108, jun 2012 .

[2] Wolfram Miekisch, Jochen K Schubert, and Gabriele F.E Noeldge-Schomburg. Diagnostic potential of breath analysisfocus on volatile organic compounds. Clinica Chimica Acta, 347(1):25-39, sep 2004.

[3] Souvik Das, Saurabh Pal, and Madhuchhanda Mitra. Significance of Exhaled Breath Test in Clinical Diagnosis: A Special Focus on the Detection of Diabetes Mellitus. Journal of medical and biological engineering, 36(5):605-624, 2016.

[4] Changjun Hou, Jincan Lei, Danqun Huo, et al. Discrimination of lung cancer related volatile organic compounds with a colorimetric sensor array. Anal. Lett., 46(13):2048, sep 2013.

[5] Immanuels Taivans, Maris Bukovskis, Gunta Strazda, et al. Breath testing as a method for detecting lung cancer. Expert Review of Anticancer Therapy, 14(2):121-123, feb 2014.

[6] Wojciech Filipiak, Anna Filipiak, Andreas Sponring, et al. Comparative analyses of volatile organic compounds (VOCs) from patients, tumors and transformed cell lines for the validation of lung cancer-derived breath markers. Journal of Breath Research, 8(2):22, may 2013.

[7] D Poli, M Goldoni, M Corradi, et al. Determination of aldehydes in exhaled breath of patients with lung cancer by means of on-fiber-derivatisation SPME-GC/MS. J Chromatogr B Analyt Technol Biomed Life Sci, 878, 2010.

[8] Meggie Hakim, Yoav Y. Broza, Orna Barash, et al. Volatile organic compounds of lung cancer and possible biochemical pathways. Chemical Reviews, 112(11):5949-5966, nov 2012.

[9] Yingchang Zou, Xi Zhang, Xing Chen, et al. Optimization of volatile markers of lung cancer to exclude interferences of non-malignant disease. Cancer Biomarkers, 14(5):371-379, jan 2014.

[10] G. de Gennaro, S. Dragonieri, F. Longobardi, et al. Chemical characterization of exhaled breath to differentiate between patients with malignant plueral mesothelioma from subjects with similar professional asbestos exposure. Anal. Bioanal. Chem., 398(7-8):3043, dec 2010.

[11] Niki Fens, Annelot G.J. van Rossum, Pieter Zanen, et al. Subphenotypes of mild-to-moderate COPD by factor and cluster analysis of pulmonary function. COPD, 10(3):277, jun 2013.

[12] M Phillips, V Basa-Dalay, J Blais, et al. Point-of-care breath test for biomarkers of active pulmonary tuberculosis. Tuberculosis, 92, 2012.

[13] Niki Fens, Aeilko H. Zwinderman, Marc P. Van Der Schee, et al. Exhaled breath profiling enables discrimination of chronic obstructive pulmonary disease and asthma. American Journal of Respiratory and Critical Care Medicine, 180(11):1076-1082, 2009. 
[14] J. J B N Van Berkel, J. W. Dallinga, G. M. Möller, et al. A profile of volatile organic compounds in breath discriminates COPD patients from controls. Respiratory Medicine, 104(4):557-563, apr 2010.

[15] Dillys van Vliet, Agnieszka Smolinska, Quirijn Jöbsis, et al. Can exhaled volatile organic compounds predict asthma exacerbations in children? Journal of Breath Research, 11(1):016016, mar 2017.

[16] Agnieszka Smolinska, Ester M. M. EM Klaassen, JW Jan W. Dallinga, et al. Profiling of volatile organic compounds in exhaled breath as a strategy to find early predictive signatures of asthma in children. PLoS ONE, 9(4):e95668, apr 2014.

[17] J. W. Dallinga, C. M H H T Robroeks, J. J B N Van Berkel, et al. Volatile organic compounds in exhaled breath as a diagnostic tool for asthma in children. Clinical and Experimental Allergy, 40(1):68-76, jan 2010.

[18] Mandy Mangler, Cornelia Freitag, Malgorzata Lanowska, et al. Volatile organic compounds (VOCs) in exhaled breath of patients with breast cancer in a clinical setting. Ginekologia polska, 83(10):730-6, oct 2012.

[19] Yiwen Xu, Hyunjoong Lee, Yushi Hu, et al. Detection and identification of breast cancer volatile organic compounds biomarkers using highly-sensitive single nanowire array on a chip. Journal of biomedical nanotechnology, 9(7):1164-72, jul 2013.

[20] S Chen, V Mahadevan, and L Zieve. Volatile fatty acids in the breath of patients with cirrhosis of the liver. The Journal of laboratory and clinical medicine, 75(4):622-7, apr 1970.

[21] A Tangerman, M T Meuwese-Arends, and J B Jansen. Cause and composition of foetor hepaticus. Lancet (London, England), 343(8895):483, feb 1994.

[22] Chris S J Probert, Iftikhar Ahmed, Tanzeela Khalid, et al. Volatile organic compounds as diagnostic biomarkers in gastrointestinal and liver diseases. Journal of gastrointestinal and liver diseases : JGLD, 18(3):337-43, sep 2009.

[23] Froukje J. Verdam, Jan W. Dallinga, Ann Driessen, et al. Non-alcoholic steatohepatitis: a non-invasive diagnosis by analysis of exhaled breath. J. Hepatol., 58(3):543-548, mar 2013.

[24] Kirsten E Pijls, Agnieszka Smolinska, Daisy M A E Jonkers, et al. A profile of volatile organic compounds in exhaled air as a potential non-invasive biomarker for liver cirrhosis. Scientific reports, 6:19903, jan 2016.

[25] A. Smolinska, A. G. L. Bodelier, J. W. Dallinga, et al. The potential of volatile organic compounds for the detection of active disease in patients with ulcerative colitis. Alimentary Pharmacology \& Therapeutics, 45(9):1244-1254, may 2017.

[26] B de Lacy Costello, A Amann, H Al-Kateb, et al. A review of the volatiles from the healthy human body. Journal of breath research, 8(1):014001, mar 2014.

[27] M R Wills and J Savory. Biochemistry of renal failure. Annals of clinical and laboratory science, 11(4):292-9.

[28] L Laffel. Ketone bodies: a review of physiology, pathophysiology and application of monitoring to diabetes. Diabetes/metabolism research and reviews, 15(6):412-426, 1999.

[29] C M Kneepkens, Guy Lepage, Claude C. Roy, et al. The potential of the hydrocarbon breath test as a measure of lipid peroxidation. Free Radical Biology and Medicine, 17(2):127-60, aug 1994.

[30] J Kwak and G Preti. Volatile disease biomarkers in breath: a critique. Curr Pharm Biotechnol, 12(7):1067-1074, 2011.

[31] Paweł Mochalski, Andreas Sponring, Julian King, et al. Release and uptake of volatile organic compounds by human hepatocellular carcinoma cells (HepG2) in vitro. Cancer Cell International, 13(1):72, jul 2013.

[32] Andreas Sponring, Wojciech Filipiak, Tomas Mikoviny, et al. Release of volatile organic compounds from the lung cancer cell line NCI-H2087 in vitro. Anticancer research, 29(1):419-26, jan 2009. 
[33] Paweł Mochalski, Ramona Al-Zoairy, Andreas Niederwanger, Karl Unterkofler, and Anton Amann. Quantitative analysis of volatile organic compounds released and consumed by rat L6 skeletal muscle cells <i in vitro</i>. Journal of Breath Research, 8(4):046003, oct 2014.

[34] Wojciech Filipiak, Andreas Sponring, Tomas Mikoviny, et al. Release of volatile organic compounds (VOCs) from the lung cancer cell line CALU-1 in vitro. Cancer Cell International, $8(1): 17$, nov 2008.

[35] Paweł Mochalski, Markus Theurl, Andreas Sponring, et al. Analysis of Volatile Organic Compounds Liberated and Metabolised by Human Umbilical Vein Endothelial Cells (HUVEC) In Vitro. Cell Biochemistry and Biophysics, 71(1):323-329, jan 2015.

[36] Agnieszka Baranska, Ettje Tigchelaar, Agnieszka Smolinska, et al. Profile of volatile organic compounds in exhaled breath changes as a result of gluten-free diet. Journal of breath research, $7(3): 037104$, sep 2013.

[37] Luca Lavra, Alexandro Catini, Alessandra Ulivieri, et al. Investigation of VOCs associated with different characteristics of breast cancer cells. Scientific Reports, 5(1):13246, oct 2015.

[38] Hossam Haick, Lu Amal, Bin-bin Ding, et al. The scent fingerprint of hepatocarcinoma: invitro metastasis prediction with volatile organic compounds (VOCs). International Journal of Nanomedicine, 7:4135, jul 2012.

[39] Kristin Schallschmidt, Roland Becker, Christian Jung, et al. Investigation of cell culture volatilomes using solid phase micro extraction: Options and pitfalls exemplified with adenocarcinoma cell lines. Journal of Chromatography B, 1006:158-166, dec 2015.

[40] Jae Sung Pyo, Hyun Kyoung Ju, Jeong Hill Park, and Sung Won Kwon. Determination of volatile biomarkers for apoptosis and necrosis by solid-phase microextraction-gas chromatography/mass spectrometry: A pharmacometabolomic approach to cisplatin's cytotoxicity to human lung cancer cell lines. Journal of Chromatography B, 876(2):170-174, dec 2008.

[41] RRR Fijten, A Smolinska, Q Shi, et al. Exposure to genotoxic compounds alters in vitro cellular VOC excretion. J Breath Res, 2017.

[42] Rachel Cavill, Danyel Jennen, Jos Kleinjans, and Jacob Jan Briedé. Transcriptomic and metabolomic data integration. Briefings in Bioinformatics, 17(5):891-901, sep 2016.

[43] Yehudit Hasin, Marcus Seldin, and Aldons Lusis. Multi-omics approaches to disease. Genome Biology, 18(1):83, dec 2017.

[44] Roger W L Godschalk, Frederik-Jan Van Schooten, and Helmut Bartsch. A critical evaluation of DNA adducts as biological markers for human exposure to polycyclic aromatic compounds. Journal of biochemistry and molecular biology, 36(1):1-11, jan 2003.

[45] Oliver Fiehn. Metabolomics-the link between genotypes and phenotypes. Plant molecular biology, 48(1-2):155-71, jan 2002.

[46] Terry L Riss, Richard A Moravec, Andrew L Niles, et al. Cell Viability Assays. Eli Lilly \& Company and the National Center for Advancing Translational Sciences, 2004.

[47] A Baranska, A Smolinska, A W Boots, J W Dallinga, and F J van Schooten. Dynamic collection and analysis of volatile organic compounds from the headspace of cell cultures. Journal of Breath Research, 9(4):047102, oct 2015.

[48] Nicolas Matheus, Sylvain Hansen, Eric Rozet, et al. An Easy, Convenient Cell and Tissue Extraction Protocol for Nuclear Magnetic Resonance Metabolomics. Phytochemical Analysis, 25(4):342-349, jul 2014.

[49] Aalim M. Weljie, , Jack Newton, Pascal Mercier, et al. Targeted Profiling: Quantitative Analysis of $1 \mathrm{H}$ NMR Metabolomics Data. 2006.

[50] A Smolinska, A-Ch C Hauschild, R R R Fijten, et al. Current breathomicsa review on data pre-processing techniques and machine learning in metabolomics breath analysis. Journal of breath research, 8(2):27105, jun 2014.

[51] G. E. P. Box and D. R. Cox. An analysis of transformations. Journal of the Royal Statistical Society. Series B (Methodological, pages 211-252, 1964. 
[52] B. Walczak and D.L. Massart. Wavelets in Chemistry, volume 22. Elsevier Science B.V, nov 2000 .

[53] Frank Dieterle, Alfred Ross, Götz Schlotterbeck, et al. Probabilistic quotient normalization as robust method to account for dilution of complex biological mixtures. Application in1H NMR metabonomics. Analytical Chemistry, 78(13):4281-4290, jul 2006.

[54] Paul H C Eilers and Hans F M Boelens. Baseline correction with asymmetric least squares smoothing. Leiden University Medical Centre Report, 1:1, 2005.

[55] Giorgio Tomasi, Frans van den Berg, and Claus Andersson. Correlation optimized warping and dynamic time warping as preprocessing methods for chromatographic data. J. Chemometr., 18(5):231, may 2004.

[56] Tim De Meyer, Davy Sinnaeve, Bjorn Van Gasse, et al. NMR-Based Characterization of Metabolic Alterations in Hypertension Using an Adaptive, Intelligent Binning Algorithm. Analytical Chemistry, 80(10):3783-3790, may 2008.

[57] L. M. T. Eijssen, M. Jaillard, M. E. Adriaens, et al. User-friendly solutions for microarray quality control and pre-processing on ArrayAnalysis.org. Nucleic Acids Research, 41(W1):W71-W76, jul 2013.

[58] M. Dai, Pinglang Wang, Andrew D. Boyd, et al. Evolving gene/transcript definitions significantly alter the interpretation of GeneChip data. Nucleic Acids Research, 33(20):e175-e175, nov 2005.

[59] B.M. Bolstad, R.A Irizarry, M. Astrand, and T.P. Speed. A comparison of normalization methods for high density oligonucleotide array data based on variance and bias. Bioinformatics, 19(2):185-193, jan 2003.

[60] Darryl Nishimura. BioCarta. Biotech Software $\mathcal{G}$ Internet Report: The Computer Software Journal for Scient, 2(3):117-120, 2001.

[61] Arthur Liberzon, Aravind Subramanian, Reid Pinchback, et al. Molecular signatures database (MSigDB) 3.0. Bioinformatics (Oxford, England), 27(12):1739-40, jun 2011.

[62] Marija Milacic, Robin Haw, Karen Rothfels, et al. Annotating Cancer Variants and Anti-Cancer Therapeutics in Reactome. Cancers, 4(4):1180-1211, nov 2012.

[63] David Croft, Antonio Fabregat Mundo, Robin Haw, et al. The Reactome pathway knowledgebase. Nucleic Acids Research, 42(D1):D472-D477, jan 2014.

[64] Thomas Kelder, Martijn P. Van Iersel, Kristina Hanspers, et al. WikiPathways: Building research communities on biological pathways. Nucleic Acids Research, 40(D1):D1301-D1307, jan 2012.

[65] Martina Kutmon, Anders Riutta, Nuno Nunes, et al. WikiPathways: Capturing the full diversity of pathway knowledge. Nucleic Acids Research, 44(D1):D488-D494, jan 2016.

[66] John Tomfohr, Jun Lu, and Thomas B Kepler. Pathway level analysis of gene expression using singular value decomposition. BMC bioinformatics, 6:225, sep 2005.

[67] Leo Breiman. Random forests. Machine Learning, 45(1):5-32, oct 2001.

[68] Bruce Thompson, Thompson, and Bruce. Canonical Correlation Analysis. In Encyclopedia of statistics in behavioral sciences. John Wiley and Sons Ltd, Chicester, UK, oct 2005.

[69] Yosef Hochberg and Yoav Benjaminit. Controlling the False Discovery Rate: A Practical and Powerful Approach to Multiple Controlling the False Discovery Rate: a Practical and Powerful Approach to Multiple Testing. Source Journal of the Royal Statistical Society. Series $B$ (Methodological) Journal of the Royal Statistical Society. Series B J. R. Statist. Soc. B, 57(1):289-300, 1995.

[70] Farshad Farshidfar, Aalim M Weljie, Karen Kopciuk, et al. Serum metabolomic profile as a means to distinguish stage of colorectal cancer. Genome Medicine, 4(5):42, may 2012.

[71] Pablo J Fernandez-Marcos and Manuel Serrano. Sirt4: the glutamine gatekeeper. Cancer cell, 23(4):427-8, apr 2013 . 
[72] Bryan T Hennessy, Debra L Smith, Prahlad T Ram, Yiling Lu, and Gordon B Mills. Exploiting the PI3K/AKT pathway for cancer drug discovery. Nature reviews. Drug discovery, 4(12):988, 2005.

[73] N. Li and J. Neu. Glutamine Deprivation Alters Intestinal Tight Junctions via a PI3-K/Akt Mediated Pathway in Caco-2 Cells. Journal of Nutrition, 139(4):710-714, feb 2009.

[74] Mariia Yuneva, Nicola Zamboni, Peter Oefner, Ravi Sachidanandam, and Yuri Lazebnik. Deficiency in glutamine but not glucose induces MYC-dependent apoptosis in human cells. The Journal of Cell Biology, 178(1), 2007.

[75] Hudson H Freeze and Alan D Elbein. Glycosylation Precursors. Cold Spring Harbor Laboratory Press, 2009.

[76] Hyung Sik Kim, Seung Jun Kwack, and Byung Mu Lee. Lipid peroxidation, antioxidant enzymes, and benzo[a]pyrene-quinones in the blood of rats treated with benzo[a]pyrene. ChemicoBiological Interactions, 127(2):139-150, jul 2000.

[77] Hojae Shim and Shang-Tian Yang. Biodegradation of benzene, toluene, ethylbenzene, and oxylene by a coculture of Pseudomonas putida and Pseudomonas fluorescens immobilized in a fibrous-bed bioreactor. Journal of Biotechnology, 67(2-3):99-112, jan 1999.

[78] Philip Newsholme, Joaquim Procopio, Manuela Maria Ramos Lima, Tania Cristina Pithon-Curi, and Rui Curi. Glutamine and glutamate-their central role in cell metabolism and function. Cell Biochemistry and Function, 21(1):1-9, mar 2003.

[79] Jenq-Lin Yang, Peter Sykora, David M Wilson, et al. The excitatory neurotransmitter glutamate stimulates DNA repair to increase neuronal resiliency. Mechanisms of ageing and development, 132(8-9):405-11, aug 2011.

[80] John P. Cooke. Asymmetrical Dimethylarginine. Circulation, 109(15), 2004.

[81] Li-Tung Huang, Chih-Sung Hsieh, Kow-Aung Chang, and You-Lin Tain. Roles of Nitric Oxide and Asymmetric Dimethylarginine in Pregnancy and Fetal Programming. International Journal of Molecular Sciences, 13(12):14606-14622, nov 2012.

[82] Josep M. Argilés, Francisco J. López-Soriano, and Sílvia Busquets. Apoptosis signalling is essential and precedes protein degradation in wasting skeletal muscle during catabolic conditions. The International Journal of Biochemistry \& Cell Biology, 40(9):1674-1678, jan 2008.

[83] Wei Han, Joell J Gills, Regan M Memmott, Stephen Lam, and Phillip A Dennis. The chemopreventive agent myoinositol inhibits Akt and extracellular signal-regulated kinase in bronchial lesions from heavy smokers. Cancer prevention research (Philadelphia, Pa.), 2(4):370-6, apr 2009.

[84] Lyn H. Jones, Arjun Narayanan, and Erik C. Hett. Understanding and applying tyrosine biochemical diversity. Molecular BioSystems, 10(5):952, apr 2014. 

CHAPTER 9

Summary \& Discussion 


\subsection{From bedside to bench}

Breathomics research is a type of omics research that involves the measurement of Volatile Organic Compounds (VOCs) in the exhaled breath [1]. The research described in this thesis revolves around the clinical use of the analysis of VOCs in respiratory conditions related to pulmonary bacterial infections and interstitial lung diseases.

The thesis starts in chapter $\mathbf{2}$ with a review of the current knowledge concerning data preprocessing and analysis that is needed for exhaled breath research.

Then a collection of chapters describes a journey from the bedside to the bench. The scientific studies described in chapters $\mathbf{3}, \mathbf{4}$ and $\mathbf{5}$ were performed at the bedside where VOCs in the exhaled breath of patients and healthy controls were sampled and measured to determine whether groups of patients could be discriminated from healthy controls. Four diseases were examined: Ventilator-Associated Pneumonia (VAP), idiopathic pulmonary fibrosis (IPF), connective tissue disease-associated interstitial lung disease (CTD-ILD), and sarcoidosis.

In these studies, two challenges were identified. The first was reproducibility of clinical exhaled breath studies, due to a lack of standardization, validation and adequate use of statistics. The second challenge was the lack of biological knowledge of the endogenous source of VOCs. The former challenge was further studied in chapter 5, where different types of validation were tested on a case study.

The latter challenge was investigated at the bench in chapters 6,7 and 8 . In chapter 6, bacteria were cultured in vitro to examine which VOCs were exclusively and collectively excreted by specific bacterial strains. Chapter 7 investigated the endogenous sources of VOCs excreted in vitro by exposing cells to a well-understood carcinogen. Finally, chapter $\mathbf{8}$ used the same set-up, but added transcriptomics and metabolomics to the breathomics data to identify associations between excreted VOCs and cellular processes to gain further knowledge of the biology behind excreted VOCs.

\subsection{General discussion}

\subsubsection{Breathomics in lung diseases}

Breathomics has the promise to be used for diagnosis and monitoring of disease. Exhaled breath contains VOCs in $\mathrm{nmol} / \mathrm{l}$ to $\mathrm{pmol} / \mathrm{l}$ concentrations in addition to the presence of nitrogen, oxygen, carbon dioxide and noble gases $[1,2]$. VOCs in the exhaled breath can originate from inhaled exogenous sources such as air pollution and vegetation, and from habits such as smoking and food intake [3, 4]. VOCs in the exhaled breath can also be produced endogenously as by-products of normal cellular metabolism and can diffuse into the alveoli from the blood or directly from the lung tissue [5]. Since the concentration of VOCs can be altered by disease and since the lungs are in direct contact with the exhaled breath, the lungs are a suitable candidate to examine the value of exhaled breath analysis.

Since 1985, hundreds of exhaled breath studies have been published on a variety of 
lung diseases, including different forms of obstructive, restrictive and infectious lung disease $[6]$.

Obstructive lung disease is characterized by airway obstruction, leading to difficulty breathing. The two main forms of obstructive lung disease are asthma and chronic obstructive pulmonary disease (COPD). The use of exhaled breath in these two forms of obstructive lung disease has been investigated frequently [7-25].

In the field of infectious lung diseases, many studies have been performed examining the headspace of bacterial cultures in vitro [26-41]. In chapter 6, we studied 4 pulmonary bacteria and were able to distinguish these based on a profile of 25 VOCs. Additionally, we were able to discriminate between methicillin-sensitive $S$. Aureus (MSSA) and methicillin-resistant S. Aureus (MRSA). Many of the identified VOCs were previously found in literature, some of which were specific for one strain, whereas others were produced by multiple strains.

Only two clinical studies were published on infectious lung disease, specifically VAP [42, 43], of which the latter reference refers to chapter 3. The second study was performed by Fowler et al. using a similar set-up to measure the exhaled breath of mechanically ventilated patients. Fowler et al. studied a group of VAP patients over time and found differential breath VOC profiles of the presence or absence of pathogens at each time point. In our VAP study, we included critically ill patients with and without VAP (32 vs. 68 patients) and found that a profile of 12 VOCs could discriminate between critically ill mechanically ventilated patients with and without VAP. The results from both studies suggest that detection of VAP in ICU patients is possible with exhaled breath analysis, enabling a simple, safe and non-invasive approach that could diminish the diagnostic burden of VAP. However, there is no overlap between the discriminating VOCs found in the two studies, and a translation from the in vitro studies towards the clinical outcomes still has to be made to link exhaled VOCs to specific bacterial infections.

The application of breathomics in restrictive lung diseases has not received much interest from the scientific community. Previously, a study was performed that measured exhaled ethane levels as a marker of various interstitial lung disease (ILD) subtypes and found higher ethane levels in ILD patients compared to healthy controls [44]. Additionally, Yamada et al. were able to distinguish between IPF patients and healthy controls using 5 exhaled VOCs [45]. Moreover, Westhoff et al. performed a pilot study with 5 patients with confirmed sarcoidosis and 4 with suspicion of sarcoidosis [46]. However, as the aim was to assess the feasibility of their method, they did not perform selection of discriminatory VOCs. As an addition to the current literature, chapters $\mathbf{4}$ and $\mathbf{5}$ describe two studies on patients with a total of three ILD subtypes. The aim of chapter 4 was to investigate whether differential diagnosis of IPF and CTD-ILD was possible. Chapter $\mathbf{5}$ used sarcoidosis as a case study to examine the effect of different types of study validation.

Various articles on the application of exhaled breath in respiratory disease have been reviewed in the introduction of this thesis and can be found in table 1.1. These studies demonstrate the proof of principle that breathomics can be used to discriminate certain group of patients. However, sample sizes are small and the results differ between studies due to using different platforms and approaches, hampering the application of exhaled breath analysis as a diagnostic tool in the clinic. 
Taking together our results and those from the literature, it seems difficult to obtain reproducible results for heterogeneous diseases such as ILD with multiple phenotypes. The challenge in this clinical field is obtaining appropriate numbers of well-phenotyped patients, setting up multicenter studies and investigating in the standardization of sampling procedures and measurement platforms.

\subsubsection{Sampling of exhaled breath}

In the exhaled breath community, a variety of sampling set-ups are used to collect the exhaled breath. Generally, these can be divided into three categories: (i) upper airway collection, (ii) alveolar air collection and (iii) mixed air collection. Each method has its advantages and disadvantages, which need to be considered carefully before choosing one over the others.

- Upper airway collection, where the dead space in the lungs is sampled, which is approximately one third of the air present in the lungs at any given time [47]. This method may be useful in the case of nitric oxide (NO) measurements, since NO is excreted into the dead space air [48].

- Alveolar air collection, where only the air is sampled that came in con- tact with the alveoli where most of the gas exchange takes place. In principle, alveolar air is the preferred lung compartment to measure in when measuring endogenously formed VOCs. In essence, the presence of dead space air would simply dilute the VOCs that are excreted in the alveolar air [49]. In order to sample the alveolar air fraction in the lungs, a $\mathrm{CO}_{2}$ monitoring device is needed to ensure that the dead space is not collected. The method is based on the fact that $\mathrm{CO}_{2}$ gas exchange only occurs in the alveoli. This results in a high $\mathrm{CO}_{2}$ concentration in the air that directly originates from the alveoli in contrast to much lower levels in the dead space air. The monitoring device will monitor the $\mathrm{CO}_{2}$ levels and will capture the air when the $\mathrm{CO}_{2}$ levels reflect that of the alveolar air. This monitoring device needs to be connected to a Tedlar ${ }^{\circledR}$ bag or gas syringe in which the air can be collected.

A recent innovation is a device developed by the breathe-free community called the RECIVA Breath sampler [50, 51], which allows researchers to sample the exhaled breath reliably. Pressure and $\mathrm{CO}_{2}$ sensors are incorporated into the device that allow sampling of specific fractions of the exhaled breath, including the alveolar air. The VOCs are immediately transferred to a thermal desorption tube without needing a (Tedlar) bag, reducing the interference of analytical artifacts. Finally, a clean air source can be incorporated to prevent interference of unwanted environmental VOCs.

- Mixed air collection, where both the alveolar air and the dead space are collected. This collection method was used in the clinical studies described in this thesis, since no a priori restrictions need to be set, so there is no need for sophisticated equipment. Moreover, although the simplicity of this method may introduce confounders, their distribution will be equally spread among patients and should 
thus not introduce significant differences. Mixed air sampling is therefore more suitable for a dedicated clinical application.

\subsubsection{The use of Tedlar ${ }^{\circledR}$ bags and thermal desorption}

Off-line MS-based methods collect the exhaled breath at a different location than the measurement equipment [52]. This is for instance possible using Tedlar ${ }^{\circledR}$ bags utilized in our research. Patients breathe into these bags, after which the VOCs are preconcentrated on thermal desorption tubes that contain VOC-capturing carbon material. Use of these bags is convenient and simple as a participant simply breathes into the bag at a normal rate until the bag is full, and thus is very suitable for widespread use. Additionally, they are available in different sizes, ranging from $250 \mathrm{~mL}$ to $5 \mathrm{~L}$, allowing for selection of the appropriate volume for a specific patient population such as children.

On the other hand, on-line measurements allow for direct and time-dependent analysis of exhaled breath, but require advanced mechanical set-ups to perform the sampling. Still, on-line measurements are more suitable for use in for example a clinician's office or when real-time measurements are required. For large-scale clinical studies, off-line methods may be more suitable as Tedlar ${ }^{\circledR}$ bags are inexpensive and can thus be used for large-scale collection of exhaled breath.

Tedlar ${ }^{\circledR}$ bags do have a downside as the material excretes phenol and N,Ndimethylacetamide into the exhaled air it contains [53]. It is therefore important that the VOCs in the Tedlar $^{\circledR}$ bags are transferred to a thermal desorption tube as quickly as possible to reduce the degree of contamination. There is still a risk of identifying these two VOCs as discriminatory since the concentrations at which they are excreted are not consistent across samples [54]. For that reason, these contaminating compounds are removed in the studies described in this thesis during the pre-processing stage before the actual analysis of the data begins. However, sometimes phenol shifts in the MS chromatogram, and is thus not removed. Yet, since phenol is also produced endogenously, if discriminatory, it is likely due to this endogenous production [55]. Another potential downside of using the Tedlar ${ }^{\circledR}$ bags is that the dead space is included when no $\mathrm{CO}_{2}$ monitoring device is used. However, because the VOCs in the bag are preconcentrated, the overall sample is not diluted. Sampling mixed exhaled air has several advantages such as the simplicity of the method and the inclusion of VOCs excreted higher up in the lungs.

In order to store the exhaled breath samples for a longer time, the VOCs are transferred from the bag to a thermal desorpton tube. However, no clear consensus exists about the length of time or the temperature at which a thermal desorption tube can be stored. Temperatures used range from $-80^{\circ} \mathrm{C}$ to room temperature [52], but no evidence exists in favor of any particular temperature. Additionally, the preferred length of time to store the tubes differs between researchers, from 2-3 hours to up to a several months. Swift measurement of the samples is especially difficult in large clinical studies. However, Schee et al. found no differences in the diagnostic accuracy of samples measured after transportation and storage up to 14 days [56]. Still, the stability of captured VOCs during long-term storage has not yet been investigated. 


\subsubsection{Mass spectrometry}

A detailed comparison between the different forms of mass spectrometry has been summarized previously [57]. Briefly, SIFT-MS, PTR-MS and IMS can perform realtime measurements where the exhaled breath collected from a participant is directly injected into the mass spectrometer. GC-MS, on the other hand, is an off-line measurement that requires preconcentration of the volatiles. The limit of detection for all methods ranges from parts per billion volume to parts per trillion volume. Of all methods, GC-MS is considered the method with the highest sensitivity and specificity [57].

In our experiments, we use off-line sampling by GC - time of flight - MS (GC-tofMS) in combination with thermal desorption. First, the thermal desorption tubes are heated, releasing the VOCs captured within them. These are then separated using gas chromatography [58]. In gas chromatography, a mobile phase transports the VOCs through a column that is coated with a stationary phase. Each VOCs has a different affinity for both phases, causing the compounds to elute at a different time, known as the retention time. After this separation, the VOCs are further separated by tofMS [59] where the VOCs are accelerated by an electric field so that they obtain a certain stable amount of kinetic energy per ion. The time it takes for a compound to reach the detector is measured as the retention time. This combination of GC and tof-MS results in successful separation of the VOCs from the exhaled breath. The mass spectrometer then measures the mass spectra for each peak, so that chemical identification is possible [60].

However, it is important to note that reliable chemical identification of VOCs is difficult. For instance, co-elution of different VOCs in a chromatogram occurs when two or more compounds are not completely separated by gas chromatography and will therefore not show up as separate peaks in the chromatogram [61]. If this peak is then selected as discriminatory, it is difficult to obtain a reliable chemical identification due to mixed mass spectra. Additionally, a National Institute of Standards and Technology (NIST) library is generally used to match the mass spectra to a specific VOC, but not all measurable VOCs are present in this library, hence impeding reliable chemical identification. It is therefore important that an experienced mass spectrometrist validates the identities found in the NIST library. Morover, constructing an in-house database by measuring pure VOCs on the mass spectrometer will allow for additional confirmation of the correct identity of a VOC.

It may be of interest to propose minimum reporting standards with respect to the chemical identification of VOCs in a study, as it is difficult for a reader to determine how confident the researchers are of the identity of a VOC. Minimum reporting standards are present in other fields that require chemical identification, such as the metabolomics and proteomics fields $[62,63]$.

\subsubsection{Data preprocessing and analysis}

Chapter 2 reviews the necessary steps of data pre-processing and data analysis for GC-MS and ion-mobility spectrometer combined with a multi-capillary column (MCC-IMS). 


\subsubsection{Data preprocessing}

Since the analytical methodology can introduce non-biological artifacts in the data, it is essential to properly pre-process the data. Pre-processing of GC-MS data generally starts with log transformation for transforming heteroscedastic noise into homoscedastic noise [64, 65], which can then be removed by a noise removal algorithm. Subsequently, baseline correction and normalization are done to produce chromatograms that can be analysed together without interference from analytical disturbances. Finally, peak picking is performed to combine peaks corresponding to the same compound across multiple samples. An overview of data pre-processing methods for GC-MS is described in chapter 2.

\subsubsection{Statistical data analysis}

Statistical data analysis can be divided into three categories: univariate, bivariate and multivariate statistics.

Univariate statistics is the simplest form of statistics, which takes into account one variable at a time $[66,67]$. For breathomics, univariate statistics will test whether the mean, median or distribution of one VOC is different between two groups of participants. The advantage of univariate statistics is that it easily calculated and interpreted and is widely available, whereas the disadvantage is that it can only test one variable at a time. A p-value of 0.05 is the common significance threshold for univariate statistics. This means that there is a $5 \%$ chance that a significant p-value is a false positive result, implying that when thousands of univariate tests are calculated on a dataset, there is a high likelihood that random events appear as significant. Multiple testing correction (MTC) methods are used to reduce the chance that false positives or negatives are generated in this way [68]. Consequently, applying MTC with univariate statistics is essential.

Bivariate statistics, on the other hand, involves two variables at the same time and examines the relationship between those two variables. An example of bivariate statistics is correlation analysis [67]. In breathomics, correlation analysis can be used to determine whether the exhaled concentration of a VOC is related to a clinical parameter measured in a patient. An advantage of bivariate statistics is that relationships between two variables can be identified. Yet, the disadvantage is that only one relationship can be examined at a time.

Multivariate statistics is an extension of univariate and bivariate statistics where multiple variables are combined instead of analyzing them one-by-one [67, 69, 70]. There are several advantages to using multivariate statistics to analyse datasets containing thousands of variables. First, unsupervised methods such as principal components analysis (PCA) allow for dimension reduction of the data, resulting in fewer variables and aiding interpretation [70]. Other unsupervised multivariate techniques such as hierarchical clustering can show groupings in the data that would not be visible while utilizing univariate statistics [70]. The advantage of an unsupervised method is that it does not require any additional information such as class labels, i.e. it does not need to know to which group a sample belongs. These methods do not, however, actively discriminate between two groups of samples. For that, supervised methods are used. The advantage of these methods is that a priori knowledge such 
as class labels are specified beforehand, and the algorithm looks for the best way to separate the groups based on that knowledge. For most methods, selection of the most important variables is possible. Since thousands of VOCs can be measured at the same time in breathomics, variable selection by supervised multivariate statistics enables researchers to select a subset of VOCs that can differentiate between the study subject groups. In order to validate the performance of a multivariate model, an array of significance measures can be used, including sensitivity, specificity, likelihood ratios, receiver operating characteristic curves and overall predictions.

The advantage of multivariate statistics is that it takes into account the combined effect of VOCs. Moreover, it allows for identification of relationships between the compounds. Multivariate statistics seems the most suitable statistical method to use in exhaled breath analysis, as VOC profiles will likely be superior for the detection of disease than individuals. This is especially the case in in vitro studies, where the concentrations of excreted VOCs are lower than in the exhaled breath, and will thus most likely not be individually significant, especially after MTC. Several multivariate statistical methods that are suitable for breathomics are reviewed in chapter 2 .

Yet, the main disadvantage of supervised multivariate statistics is overfitting [71]. Overfitting is the concept where a multivariate model is created on a specific group of samples in a study that do not accurately represent the true population. When this model is applied to subjects with the same disease, but in a different study population, the model will not be able to predict those samples, making the results irreproducible. Overfitting can be prevented by applying proper (external) validation of the technique and by optimization of the procedures.

\subsubsection{The reproducibility crisis}

In 2015, the scientific community was shocked to learn that the majority of psychology studies were found irreproducible [72]. Reproducibility in science is a concept that states that when studies on the same subject or problem are performed, the same or similar results should be found. This "reproducibility crisis" does not limit itself to psychology research, but extends also to the biomedical field, including omics research $[73,74]$. In breathomics, a similar pattern emerges. A handful of systematic reviews have been published that compare the outcome of different studies on similar diseases. These reviews found little overlap in the identified VOCs between the studies. For example, van de Kant et al. [75] included a total of 73 studies on lung cancer, COPD, asthma, cystic fibrosis ( $\mathrm{CF}$ ), tuberculosis (TB), acute respiratory distress syndrome (ARDS) and ILD. Lung cancer was mainly represented, with 34 included articles. Of the 118 VOCs identified in the 73 studies, $53 \%$ were found only once in a disease. For lung cancer, 57 VOCs (48\%) were found more than once. For the other diseases, only a handful of VOCs were found in multiple studies: three VOCs for ARDS and $\mathrm{CF}$, and two VOCs for asthma. Only eight VOCs were found in more than 5 studies, all of which were found in lung cancer. Consequently, these 8 most promising VOCs were found in only $15 \%$ of all included lung cancer studies. In two other systematic reviews, a $14 \%$ and $7 \%$ overlap was found in discriminatory volatiles for COPD [76] and asthma [77] respectively.

This lack of reproducibility in breathomics can be attributed to a number of 
factors. First and foremost, the studied diseases are complex and affect the entire metabolism, so small differences in patient phenotypes between studies may change the exhaled VOC profiles. Other factors that play a role include differences in study design, technology platforms, sampling, clinical phenotyping, a lack of standardized protocols, selective reporting of positive findings, misinterpretation of non-biologically introduced noise and poor documentation [78]. This combination between complex diseases and the above-mentioned differences between studies results in identification of different sets of biomarkers that are predictive of disease. Breathomics researchers deal with extremely complex datasets that contain thousands of variables, where bias can be introduced at every step, starting from sampling up to the data analysis. It is therefore of the utmost importance that counter measures are taken to ensure reproducibility of breathomics research.

Two examples of these counter measures will be described below: standardization and study validation.

\subsubsection{Standardization}

The volatile organic compounds in our exhaled breath are easily influenced by subtle changes in the environment or differences between subjects in a study. For instance, Blanchet et al. [79] found that gender, age, BMI and smoking behaviour significantly influenced the pattern of VOCs in the exhaled breath. Controlling for variation in sampling methodology is also important as it was shown that ethanol and acetone levels fluctuated with different expiratory flow rates [80]. It is also known that specific diets influence the exhaled breath [81, 82], where Baranska et al. [82] showed that gluten-free diet altered the pattern of exhaled VOCs, which reverted back to the old state when the gluten-free diet was halted. In addition to biological variation, analytical variation can introduce bias. As is described above, different sampling methods exist, for instance alveolar air versus mixed air sampling or off-line versus on-line measurements. Also, different mass spectrometer set-ups can introduce bias in the chromatograms. Yet, inter-laboratory comparisons have not yet been performed by the exhaled breath community.

All of these pieces of evidence point to one thing: standardization is a factor that needs to be considered carefully during the designing stage of a breathomics study. Improving standardization can be relatively straight-forward and has been done for other omics fields. For instance, standard operating procedures (SOPs) for genome annotations for a gene's name, or biological function have been established in 2008 [83]. For metabolomics, SOPs exists for pre-analytical handling of blood and urine [84]. No such thing has been done yet for the field of breathomics, yet could have great value.

\subsubsection{Study validation}

In order to ensure reproducibility of an individual study, it is important to add some form of statistical validation. There are many forms of statistical validation, which can be divided into internal and external validation.

Internal validation is a type of statistical validation where part of a collected dataset is set aside to validate a model. The model is created on the remaining data 
and is projected onto the data that was set aside. Several types of internal validation exist. Leave-one-out and leave-k-out cross validation both greatly overestimate the predictive capacity of the model and thus do not give a correct estimate of the reproducibility of a study [85]. Bootstrapping, where a subset is set aside and a model is created many times with replacement, gives the most reliable estimate of the internal validation methods [85]. However, if the study population does not reflect the true patient population, internal validation will not be enough.

For that reason, external validation is essential. For true external validation, a dataset is needed from a different study. For instance, the predictive model is created on a study from one hospital, and is validated on a study from a different hospital, but with the same patient population [86]. This approach was taken in chapter 5, where VOCs were selected based on one study and validated in another. The predictive power of different validation methods was also reported.

\subsubsection{The biological origin of VOCs}

Another challenge in the field of breathomics is that the biological source of most discriminatory VOCs is not known. A complicating factor is that many exhaled VOCs are exogenously produced by pollution, plants and bacteria [26, 87-89]. These VOCs can enter the body through inhalation and afterwards end up in the exhaled breath.

A lot of research has been performed on the endogenous sources of VOCs excreted by different disease-causing bacteria [27-41]. Chapter 6 also examined the VOCs excreted by five bacterial strains commonly found in ventilated patients in the intensive care unit. Several strain-specific VOCs were discovered, and some were commonly found derived from multiple strains. The hypothesized origins of VOCs produced by bacteria fall into the following categories: (i) hydrocarbons produced via the methylerythritol phosphate pathway or $\beta$-oxidation of fatty acids, (ii) alcohols produced through the oxidation of fatty acids, (iii) acids due to anaerobic metabolism, (iv) aldehydes derived from oxidative metabolism, (v) ketones produced during decarboxylation of fatty acid derivatives, (vi) cyclic compounds of which the biological origin remains largely unknown, (vii) esters as a result of esterification between acetic acid and alcohols, (viii) S-containing compounds mostly of unknown origin and (ix) $\mathrm{N}$-containing compounds resulting from amino acid breakdown [26, 40, 41].

In contrast, the endogenous human source of only a handful of VOCs is known. For instance, dimethylsulfide is responsible for fetor hepaticus, also known as breath of the dead which is a late sign of liver failure [90, 91]. Another example is acetone, which is exhaled during ketoacidosis as a result of diseases like diabetes and sepsis $[92,93]$. For many other VOCs, the endogenous sources have been hypothesized, but have not been proven. Hydrocarbons are thought to be produced by oxidative stress and lipid peroxidation as a result of disease [94], whereas the hypothetical source of aldehydes is normal physiological processes including gene regulation, proliferation and signal transduction [95]. In chapters $\mathbf{7}$ and $\mathbf{8}$, we have attempted to identify putative targets of endogenous production of specific VOCs. Both chapters have pointed to several likely endogenous sources of VOCs. Nonanal was identified as the most promising candidate biomarker for exposure to genotoxic chemicals due to its 
correlation to apoptosis and proliferation pathways in chapter $\mathbf{8}$.

\subsection{Conclusion and future perspectives}

In conclusion, this thesis describes the progression of exhaled breath research from bedside to bench, starting with clinical studies to diagnose lung diseases and ending with dedicated in vitro experiments to elucidate the endogenous sources of exhaled VOCs. In the discussion of this thesis, several challenges were addressed that need to be faced in breathomics. Reproducibility needs to be ensured by more stringent standardization through establishing SOPs and by using external validation. Multivariate statistics with predictive modelling in combination with external validation will enable researchers to examine the validity of the identified VOCs. Finally, gaining more biological insight in the endogenous sources of exhaled VOCs will enable researchers to connect these to biological processes of disease. It thus will allows for disease monitoring instead of diagnosis alone. However, a collective effort is needed from the entire community to meet these challenges and to ensure that exhaled breath analysis can one day be used to help patients.

\subsection{Main Findings}

\section{Exhaled breath analysis can discriminate critically ill patients with and without VAP}

The aim of the study in chapter 3 was to identify VOCs that could discriminate between critically ill patients with VAP and those without VAP.

\section{Summary of the main findings of the thesis}

- Distinction between patients with and without VAP was $74.2 \%$ accurate with 12 VOCs in the exhaled breath. One other published study reported an accuracy of $98 \%$, but examined a less heterogeneous group [42]. Bronchoalveolar lavage is the golden standard, but is laborious, time-consuming and takes up to 48 hours before definitive results are available. Exhaled breath analysis, a non-invasive and relatively fast technique, may therefore be suitable for clinical diagnosis of VAP.

- Likely sources of other VOCs were identified using pathway analysis and literature. The KEGG database encompasses 520,153 pathways for 5,257 organisms (of which 4,290 bacteria) that mainly focus on metabolism [96]. Most common infectious bacteria are present in the database, so VOCs produced in a metabolic pathway can be traced back to a specific strain. For instance, ethanol and 2propenal were found to be linked to bacterial and human metabolism. The likely source of the (branched) alkanes heptane, 2-methylbutane, dodecane, tetradecane and tetradecanal were found using literature research. These were produced as a result of oxidative stress and lipid peroxidation [94]. Additionally, 
ethylbenzene, tetrahydrofuran and caraneSeveral were identified as pollutionderived VOCs. Other discriminatory VOCs likely originated from the host, such as acetone, which was elevated in VAP patients. It is produced due to build-up of ketone bodies, which was found previously occurs during sepsis [93], suggesting that the exhaled acetone was due to varying degrees of sepsis in these patients.

\section{Implications}

The current golden standard, bronchoalveolar lavage, for VAP diagnosis is timeconsuming and invasive. Additionally, antibiotic resistance is a rapidly emerging problem, especially in the ICU. Both of these problems may be solved by the use of exhaled breath analysis, which could result in earlier diagnosis of VAP in critically ill patients. This earlier diagnosis could reduce the use of preventive antibiotics and thus slow down the emergence of multidrug resistant microorganisms. The findings of this study are promising, but need to be validated in a larger study population. Additionally, determining whether exhaled VOCs can distinguish different bacterial infections would be extremely valuable, as it would allow clinicians to provide patients with tailored antibiotic treatments, thus once more possibly slowing down the rise in antibiotic resistance.

\section{Exhaled breath can distinguish between idiopathic pulmonary fibrosis and ILD-CTD, but the results may not be replicable in an independent validation study}

Differential diagnosis of interstitial lung diseases (ILD) is difficult, warranting a noninvasive and quick method to achieve this. Chapter 4 describes discrimination between idiopathic pulmonary fibrosis (IPF) and connective tissue disease-associated ILD (CTD-ILD).

\section{Main findings and discussion}

- A discriminative profile of 16 VOCs could distinguish between 51 IPF and 53 $C T D$-ILD patients. The multivariate model achieved a predictive accuracy of $76.9 \%$. Of these VOCs, several are associated with the specific forms of ILD. For instance, heptane was decreased in the breath of IPF patients compared to the CTD-ILD patients, suggesting a role for this VOC in the pathology of IPF. Three other VOCs of interest were dimethylsulfide and dimethylsulfone. Dimethylsulfide was decreased in IPF vs. healthy controls, whereas the level of dimethylsulfone increased in IPF compared to CTD-ILD. The presence of these two volatiles can be explained by enhanced production of hydrogen peroxide by NADPH oxidase 4, activated by transforming growth factor which is considered the hallmark of IPF.

- VOCs were identified that could discriminate between both diseases and healthy controls. A profile of 34 VOCs could discriminate between IPF patients and healthy controls, whereas 11 unique VOCs were able to distinguish CTD-ILD 
patients from healthy controls. Only one VOC overlapped between these two comparisons, namely ethanol, which was decreased in both forms of ILD. It likely originates from the human body, where its formation in the human body is influenced by various physiological circumstances and can be hampered by both ageing and oxidative stress, two conditions frequently reported to be associated with ILDs in general and IPF specifically.

- Associations were found between the IPF vs. CTD-ILD VOC profile and total lung capacity and the 6 minutes walking distance. Interestingly, Yamada et al. found similar associations between exhaled VOCs and clinical lung function parameters, underlining the usefulness of breathomics in the diagnosis of ILD.

- These findings may not be reproducible as internal validation was not achieved. However, due to the heterogeneous nature of ILD, the patient numbers may not have been large enough. The majority of VOCs in each of the 3 discriminatory models only reached a statistically significant power when the number of individuals in each group would be 6 to 7 times larger. These findings suggest that larger participant groups may have allowed for successful validation, but are no guarantee.

\section{Implications}

This study reports for the first time that differentiation of IPF and CTD-ILD as well as distinguishing both diseases from healthy controls. Additionally, an ILD-specific VOC profile was correlated to clinical parameters. However, due to the heterogeneous nature of ILD, larger studies are warranted to validated these findings and to facilitate fast, non-invasive and proper differential diagnosis of specific ILDs in the future.

\section{External validation is essential to ensure reproducibility in ex- haled breath studies}

Reproducibility is a major issue in exhaled breath research. In chapter $\mathbf{5}$, sarcoidosis was used as a case study to examine the use of different types of validation, especially independent external validation, and their effect on the reproducibility of the findings.

\section{Main findings and discussion}

- Nine VOCs could discriminate sarcoidosis patients from healthy controls with an accuracy of $79.4 \%$. At present, only a small-scale feasibility study has been published on sarcoidosis, but did not select discriminatory VOCs or report a predictive accuracy [46].

- A comparison of different types of validation demonstrated that internal validation methods such as leave-k-out produced the most optimistic prediction, whereas external validation had the least optimistic prediction. This was in concordance with previous research that has shown that leave-1-out crossvalidation is an incomplete measure and overestimation of the predictive power of a model [85]. Bootstrapping is the most robust internal validation method. 
However, external validation remains the best representation of the true predictive power of a model [85].

- Indirect external validation was achieved, but with low confidence. Direct projection of the classification model was not successful due to various differences between the discovery and validation study, such as differences in time and location, analytical differences between the discovery and validation study, or a combination of these factors. Indirect external validation was performed by creating a new model for the previously selected discriminatory VOCs, and demonstrated a discriminatory accuracy of $74.1 \%$, indicating that the selected VOCs did have some informative value. However, the confidence of the predictive model was not optimal.

- Only isoprene could be traced back to a putative endogenous source using literature research. Isoprene is produced by the mevalonate pathway and is likely related to inactivity due to sarcoidosis-associated fatigue [97, 98]. Conflicting evidence was found for the other discriminatory VOCs. Most were exhaled in lower concentrations by patients, but it is therefore unclear what the sources of these VOCs are.

\section{Implications}

This study has demonstrated the necessity of using proper validation methods, preferably external independent validation, in clinical exhaled breath research. It is therefore highly recommended to include an external validation dataset in future studies to ensure reproducibility.

\section{Strain-specific VOCs were found in vitro}

The in vitro study in chapter 6 identified VOCs produced by bacteria that frequently cause pulmonary infections and exacerbations of various lung diseases including VAP [99].

\section{Main findings and discussion}

- Four strains of bacteria could be distinguished from one another with 25 VOCs found in the headspace of the bacterial cultures. Fifteen of these were specific for one strain, whereas the other 10 were found in more than one strain. Seven of the discriminatory VOCs were hydrocarbons that may be produced as a result of lipid peroxidation by bacteria [100]. Five other VOCs were classified as ketone bodies and were also previously identified in the headspace of $P$. aeruginosa and $S$. aureus $[40,41,101-105]$. Finally, three aromatic hydrocarbons and three aldehydes were identified. The aromatic hydrocarbons were not found previously in bacterial cultures, whereas the aldehydes were excreted previously by E. coli, P. aeruginosa and K. pneumoniae [104, 106].

- A group of 8 VOCs could discriminate between methicillin-sensitive S. Aureus (MSSA) and methicillin-resistant S. Aureus (MRSA). These include three 
chlorine-containing compounds, of which the origin needs to be determined. Others include toluene, an environmental pollutant, and two ketones, produced by fatty acid decarboxylation [39].

\section{Implications}

These findings demonstrate the ability to identify specific bacterial strains based on a limited group of VOCs in the in vitro headspace. This method could be applied to aid clinical identification of bacteria, but may also be translatable to an in vivo situation where exhaled breath could be used to identify specific infections in VAP, as described in chapter 3. However, no overlap was found in the VOCs identified in this chapter and the ones in chapter 3 .

\section{VOCs are differently abundant in headspace of pulmonary cells exposed to genotoxic compounds in vitro}

Chapter 7 demonstrated that the genotoxic chemicals benzo[a]pyrene (B[a]P) and cisplatin elicit a cellular response in vitro, resulting in altered concentrations of excreted VOCs in the headspace of two pulmonary cell lines.

\section{Main findings and discussion}

- $B[a] P$ exposure resulted in a cellular response with 6 differentially excreted VOCs, whereas 8 VOCs were differentially excreted after cisplatin exposure. Three of the differentially excreted VOCs belonged to the class of alkanes. The presence of alkanes is thought to originate from lipid peroxidation induced by both genotoxic chemicals [94]. Additionally, two aldehydes were identified, which are produced as by-products of oxidative metabolism [95].

- The aldehyde nonanal was found in both exposures. The concentration of nonanal was increased after both exposures and was found previously by Pyo et al. [107] who also exposed pulmonary cells to cisplatin. This compound may therefore be a likely candidate for further investigation for use as a biomarker of genotoxicity-induced apoptosis.

\section{Implications}

The study has shown that genotoxic chemicals elicit a response from cells that are reflected by the excreted VOCs in the headspace of exposed cells. Nonanal especially is of interest as it was found in both exposures. Additionally, the strategies described in this chapter could be used as a guideline for future in vitro research on VOCs.

\section{In vitro integrative analysis of transcriptomics, metabolomics and breathomics identifies likely endogenous sources of VOCs}

The final chapter, chapter 8, aimed to identify putative endogenous sources of VOCs by collecting transcriptomics, metabolomics and breathomics data. Integration and subsequent correlation analysis of the data was done to find these endogenous sources. 


\section{Main findings and discussion}

- Six VOCs, 7 intracellular metabolites (ICMs) and 71 pathways were identified as discriminatory between exposed and non-exposed cells. The pathways were divided into 9 KEGG pathways, 10 BioCarta pathways, 19 WikiPathways and 33 Reactome pathways. The BioCarta pathways are mainly characterized by apoptosis and proliferation pathways that revolve around the $\mathrm{Pi} 3 \mathrm{~K} / \mathrm{AKT}$ pathway. The KEGG pathways mainly include metabolism pathways like oxidative phosphorylation and metabolism of n-glycans. The Reactome pathways include many DNA repair and cancer-related pathways. Finally, the WikiPathways database found pathways related to cell cycle, apoptosis and DNA damage response.

- Canonical Correlation Analysis (CCA) revealed significant correlations between the three omics datasets. CCA analysis of VOCs and pathways did not yield significant correlations. The comparisons between VOCs and ICMs, and ICMs and pathways did yield significant correlations, with a minimum correlation coefficient of 0.93 .

- Correlations between individual VOCs, ICMs and pathways identified clusters of pathways associated with a certain metabolite, which in turn was associated with a certain VOC. These associations were combined into a network to visualize the entire data structure, which was subsequently used to generate hypotheses for the origin of the identified VOCs. For example, octadecane was negatively associated with glutamine, which in turn was positively correlated to the pathways in WikiPathways cluster 1 and Reactome cluster 1 . These clusters contain cancer-related, dna repair, apoptosis and cell cycle pathways.

- Literature research was performed to validate the putative endogenous sources of specific VOCs. For instance nonanal, which was found as discriminatory after exposure to two carcinogenic chemicals in chapter 7, was negatively correlated with myo-inositol, which in turn was correlated to most BioCarta pathways. These pathways are mainly based on the Akt/Pi3K pathway, which is up-regulated, thus promoting proliferation [108]. It is therefore hypothesized that the level of myo-inositol is upregulated as a compensation mechanism for overproliferation since it can inhibit the Akt/Pi3K pathway [109]. Nonanal may therefore be produced as a result of this compensation mechanism.

\section{Implications}

Several putative endogenous sources were identified for five of the six discriminatory VOCs. However, it is important to note that association does not equal causation. Wet-lab validation of these findings is therefore essential. Causative relations can only be found by measuring headspace VOCs after manipulation of the proposed VOC producing pathways, using for instance molecular techniques to up- or downregulate specific pathways using RNAi or CRISPR-Cas9 [110, 111]. 


\section{Bibliography}

[1] Agnes W Boots, Joep J B N van Berkel, Jan W Dallinga, et al. The versatile use of exhaled volatile organic compounds in human health and disease. Journal of Breath Research, $6(2): 27108$, jun 2012.

[2] Wolfram Miekisch, Jochen K Schubert, and Gabriele F.E Noeldge-Schomburg. Diagnostic potential of breath analysisfocus on volatile organic compounds. Clinica Chimica Acta, 347(1):2539, sep 2004.

[3] Ankur Kansal. Sources and reactivity of NMHCs and VOCs in the atmosphere: A review. Journal of Hazardous Materials, 166(1):17-26, jul 2009.

[4] W Filipiak, V Ruzsanyi, P Mochalski, et al. Dependence of exhaled breath composition on exogenous factors, smoking habits and exposure to air pollutants. J Breath Res, 6, 2012.

[5] Marc Philippe Van Der Schee, Tamara Paff, Paul Brinkman, et al. Breathomics in lung disease. Chest, 147(1):224-231, jan 2015.

[6] Andrew J. Lechner, George M. Matuschak, and David S. Brink. Respiratory : an integrated approach to disease.

[7] J.J.B.N. Van Berkel, J.W. Dallinga, G.M. Möller, et al. Development of accurate classification method based on the analysis of volatile organic compounds from human exhaled air. Journal of Chromatography B, 861(B, 861):101-107, jan 2008.

[8] Diana Poli, Paolo Carbognani, Massimo Corradi, et al. Exhaled volatile organic compounds in patients with non-small cell lung cancer: cross sectional and nested short-term follow-up study. Respir Res, 6(1):71, dec 2005.

[9] P Paredi, S A Kharitonov, and P J Barnes. Elevation of exhaled ethane concentration in asthma. Am J Respir Crit Care Med, 162, 2000.

[10] Christopher Phillips, Neil Mac Parthaláin, Yasir Syed, et al. Short-Term Intra-Subject Variation in Exhaled Volatile Organic Compounds (VOCs) in COPD Patients and Healthy Controls and Its Effect on Disease Classification. Metabolites, 4(2):300-18, may 2014.

[11] José Javier Jareño-Esteban, M. Ángeles Muñoz-Lucas, Óscar Gómez-Martín, et al. Study of 5 Volatile Organic Compounds in Exhaled Breath in Chronic Obstructive Pulmonary Disease. Archivos de Bronconeumología (English Edition), 53(5):251-256, may 2017.

[12] A Gaida, O Holz, C Nell, et al. A dual center study to compare breath volatile organic compounds from smokers and non-smokers with and without COPD. Journal of Breath Research, 10(2):026006, apr 2016. 
[13] M Allers, J Langejuergen, A Gaida, et al. Measurement of exhaled volatile organic compounds from patients with chronic obstructive pulmonary disease (COPD) using closed gas loop GCIMS and GC-APCI-MS. Journal of Breath Research, 10(2):026004, apr 2016.

[14] Vasiliki Besa, Helmut Teschler, Isabella Kurth, et al. Exhaled volatile organic compounds discriminate patients with chronic obstructive pulmonary disease from healthy subjects. International journal of chronic obstructive pulmonary disease, 10:399-406, 2015.

[15] Maria Basanta, Baharudin Ibrahim, Rachel Dockry, et al. Exhaled volatile organic compounds for phenotyping chronic obstructive pulmonary disease: a cross-sectional study. Respiratory research, 13(1):72, aug 2012.

[16] M Phillips, V Basa-Dalay, J Blais, et al. Point-of-care breath test for biomarkers of active pulmonary tuberculosis. Tuberculosis, 92, 2012.

[17] A.-C. Hauschild, J.I. I Baumbach, and J.I. I Baumbach. Integrated statistical learning of metabolic ion mobility spectrometry profiles for pulmonary disease identification. Genetics and Molecular Research, 11(3):2733, aug 2012.

[18] Dillys van Vliet, Agnieszka Smolinska, Quirijn Jöbsis, et al. Can exhaled volatile organic compounds predict asthma exacerbations in children? Journal of Breath Research, 11(1):016016, $\operatorname{mar} 2017$.

[19] E van Mastrigt, A Reyes-Reyes, K Brand, et al. Exhaled breath profiling using broadband quantum cascade laser-based spectroscopy in healthy children and children with asthma and cystic fibrosis. Journal of Breath Research, 10(2):026003, apr 2016.

[20] Ester M. M. Klaassen, Kim D. G. van de Kant, Quirijn Jöbsis, et al. Exhaled Biomarkers and Gene Expression at Preschool Age Improve Asthma Prediction at 6 Years of Age. American Journal of Respiratory and Critical Care Medicine, 191(2):201-207, jan 2015.

[21] Norbert Meyer, Jan W Dallinga, Sarah Janine Nuss, et al. Defining adult asthma endotypes by clinical features and patterns of volatile organic compounds in exhaled air. Respiratory research, 15(1):136, nov 2014.

[22] Agnieszka Smolinska, Ester M. M. EM Klaassen, JW Jan W. Dallinga, et al. Profiling of volatile organic compounds in exhaled breath as a strategy to find early predictive signatures of asthma in children. PLoS ONE, 9(4):e95668, apr 2014.

[23] Charlotte M. Robroeks, Joep J. Van Berkel, Quirijn Jöbsis, et al. Exhaled volatile organic compounds predict exacerbations of childhood asthma in a 1-year prospective study. European Respiratory Journal, 42(1):98-106, 2013.

[24] Florian Gahleitner, Cristina Guallar-Hoyas, Caroline S Beardsmore, Hitesh C Pandya, and CL Paul Thomas. Metabolomics pilot study to identify volatile organic compound markers of childhood asthma in exhaled breath. Bioanalysis, 5(18):2239-2247, sep 2013.

[25] M. Caldeira, R. Perestrelo, A.S. S Barros, et al. Allergic asthma exhaled breath metabolome: A challenge for comprehensive two-dimensional gas chromatography. J Chromatogr A, 1254:8797, sep 2012.

[26] Lieuwe D. J. Bos, Peter J. Sterk, Marcus J. Schultz, L Hofmann, and S Ulbricht. Volatile Metabolites of Pathogens: A Systematic Review. PLoS Pathogens, 9(5):e1003311, may 2013.

[27] Fraser Lough, John D. Perry, Stephen P. Stanforth, and John R. Dean. Detection of exogenous VOCs as a novel invitro diagnostic technique for the detection of pathogenic bacteria. $\operatorname{Tr} A C$ Trends in Analytical Chemistry, 87:71-81, 2017.

[28] David Smith, Patrik Španěl, Francis J Gilchrist, et al. Hydrogen cyanide, a volatile biomarker of Pseudomonas aeruginosa infection. Journal of Breath Research, 7(4):044001, dec 2013.

[29] Violetta Shestivska, Kseniya Dryahina, Jaroslav Nunvá, et al. Quantitative analysis of volatile metabolites released $<\mathrm{i}>$ in vitro $</ \mathrm{i}>$ by bacteria of the genus $<\mathrm{i}>$ Stenotrophomonas $</ \mathrm{i}>$ for identification of breath biomarkers of respiratory infection in cystic fibrosis. Journal of Breath Research, 9(2):027104, apr 2015.

[30] Roman Purkhart, Gunther Becher, Petra Reinhold, and Heike Köhler. Rapid detection of mycobacterial growth in in vitro cultures by VOC analysis. European Respiratory Journal, 44(Suppl 58), 2014. 
[31] F. J. Gilchrist, P. Španěl, D. Smith, and W. Lenney. The in vitro identification and quantification of volatile biomarkers released by cystic fibrosis pathogens. Anal. Methods, 7(3):818-824, 2015.

[32] Roman Purkhart, Gunther Becher, Petra Reinhold, and Heike U Köhler. Detection of mycobacteria by volatile organic compound analysis of in vitro cultures using differential ion mobility spectrometry. Journal of Medical Microbiology, 66(3):276-285, mar 2017.

[33] Michael Phillips, Gavin R Flematti, Timothy ME Davis, et al. Detection of carbon disulfide in breath and air: a possible new risk factor for coronary artery disease. International Archives of Occupational and Environmental Health, 64(2):119-123, aug 1992.

[34] K D Nizio, K A Perrault, A N Troobnikoff, et al. $<$ i $>$ In vitro $</$ i $>$ volatile organic compound profiling using GCGC-TOFMS to differentiate bacteria associated with lung infections: a proof-of-concept study. Journal of Breath Research, 10(2):026008, apr 2016.

[35] Kseniya Dryahina, Kristýna Sovová, Alexandr Nemec, and Patrik Španěl. Differentiation of pulmonary bacterial pathogens in cystic fibrosis by volatile metabolites emitted by their in vitro cultures: Pseudomonas aeruginosa, Staphylococcus aureus, Stenotrophomonas maltophilia and the Burkholder. Journal of Breath Research, 10(3):037102, aug 2016.

[36] A H Neerincx, B P Geurts, M F J Habets, et al. Identification of <i >Pseudomonas aeruginosa $</ \mathrm{i}>$ and $<\mathrm{i}>$ Aspergillus fumigatus $</ \mathrm{i}>$ mono- and co-cultures based on volatile biomarker combinations. Journal of Breath Research, 10(1):016002, jan 2016.

[37] Rina PM Wong, Gavin R Flematti, and Timothy ME Davis. Investigation of volatile organic biomarkers derived from Plasmodium falciparum in vitro. Malaria Journal, 11(1):314, sep 2012.

[38] M. E. Dolch, C. Hornuss, C. Klocke, et al. Volatile organic compound analysis by ion molecule reaction mass spectrometry for Gram-positive bacteria differentiation. European Journal of Clinical Microbiology \& Infectious Diseases, 31(11):3007-3013, nov 2012.

[39] a W Boots, A Smolinska, J.J.B.N. van Berkel, et al. Identification of microorganisms based on gas chromatography-mass spectrometric analysis of volatile organic compounds in headspace gases. Journal of biomedicine E biotechnology, 8(2):1752, jun 2014.

[40] Wojciech Filipiak, Andreas Sponring, Maria M. Baur, et al. Characterization of volatile metabolites taken up by or released from Streptococcus pneumoniae and Haemophilus influenzae by using GC-MS. Microbiology (United Kingdom), 158(12):3044-3053, dec 2012.

[41] Wojciech Filipiak, Andreas Sponring, Maria Magdalena Baur, et al. Molecular analysis of volatile metabolites released specifically by staphylococcus aureus and pseudomonas aeruginosa. BMC Microbiology, 12(1):113, jun 2012.

[42] S. J. Fowler, M. Basanta-Sanchez, Y. Xu, R. Goodacre, and P. M. Dark. Surveillance for lower airway pathogens in mechanically ventilated patients by metabolomic analysis of exhaled breath: a case-control study. Thorax, 70(4):320-325, apr 2015.

[43] Ronny Schnabel, Rianne Fijten, Agnieszka Smolinska, et al. Analysis of volatile organic compounds in exhaled breath to diagnose ventilator-associated pneumonia. Scientific Reports, 5(October):17179, jan 2015.

[44] Soichiro Kanoh, Hideo Kobayashi, and Kazuo Motoyoshi. Exhaled Ethane. Chest, 128(4):23872392 , oct 2005 .

[45] Yu-ichi Yamada, Gen Yamada, Mitsuo Otsuka, et al. Volatile Organic Compounds in Exhaled Breath of Idiopathic Pulmonary Fibrosis for Discrimination from Healthy Subjects. Lung, 195(2):247-254, apr 2017.

[46] M Westhoff, P Litterst, L Freitag, and J I Baumbach. Ion mobility spectrometry in the diagnosis of sarcoidosis: results of a feasibility study. Journal of physiology and pharmacology : an official journal of the Polish Physiological Society, 58 Suppl 5(Pt 2):739-51, nov 2007.

[47] John B. (John Burnard) West and Andrew Luks. West's respiratory physiology : the essentials.

[48] L E Gustafsson, A M Leone, M G Persson, N P Wiklund, and S Moncada. Endogenous nitric oxide is present in the exhaled air of rabbits, guinea pigs and humans. Biochemical and biophysical research communications, 181(2):852-7, dec 1991. 
[49] Wolfram Miekisch, Sabine Kischkel, Annika Sawacki, et al. Impact of sampling procedures on the results of breath analysis. Journal of Breath Research, 2(2):026007, jun 2008.

[50] Breathe Free - A community of researchers interested in breath based diagnostics, jointly designing an open-source breath sampler that can be used with a range of analytical instruments.

[51] Marc van der Schee and Theodore W Wilson. Breath Diagnostics. biomarkers, 2:3.

[52] Jonathan Beauchamp. Current sampling and analysis techniques in breath researchresults of a task force poll. Journal of Breath Research, 9(4):047107, nov 2015.

[53] Jonathan Beauchamp, Jens Herbig, Rene Gutmann, and Armin Hansel. On the use of Tedlar $\mathbb{R}$ bags for breath-gas sampling and analysis. Journal of Breath Research, 2(4):046001, dec 2008.

[54] David Beale, Oliver Jones, Avinash Karpe, et al. A Review of Analytical Techniques and Their Application in Disease Diagnosis in Breathomics and Salivaomics Research. International Journal of Molecular Sciences, 18(1):24, dec 2016.

[55] RRR Fijten, A Smolinska, Q Shi, et al. Exposure to genotoxic compounds alters in vitro cellular VOC excretion. J Breath Res, 2017.

[56] M P van der Schee, N Fens, P Brinkman, et al. Effect of transportation and storage using sorbent tubes of exhaled breath samples on diagnostic accuracy of electronic nose analysis. Journal of Breath Research, 7(1):016002, dec 2012.

[57] Célia Lourenço and Claire Turner. Breath analysis in disease diagnosis: methodological considerations and applications. Metabolites, 4(2):465-98, jun 2014.

[58] Harold Monroe McNair and James M. Miller. Basic gas chromatography. John Wiley \& Sons, 2009.

[59] Edward William Schlag. Time-of-flight mass spectrometry and its applications. Elsevier, 1994.

[60] Jan W. Dallinga, Agnieszka Smolinska, and Frederik-Jan J van Schooten. Analysis of Volatile Organic Compounds in Exhaled Breath by Gas Chromatography-Mass Spectrometry Combined with Chemometric Analysis. In Mass Spectrometry in Metabolomics, volume 1198, pages 251-263. Humana Press, New York, NY, 2014/10/02 edition, 2014.

[61] Mehdi Jalali-Heravi and Hadi Parastar. Assessment of the co-elution problem in gas chromatography-mass spectrometry using non-linear optimization techniques. Chemometrics and Intelligent Laboratory Systems, 101(1):1-13, mar 2010.

[62] Lloyd W Sumner, Alexander Amberg, Dave Barrett, et al. Proposed minimum reporting standards for chemical analysis Chemical Analysis Working Group (CAWG) Metabolomics Standards Initiative (MSI). Metabolomics : Official journal of the Metabolomic Society, 3(3):211221 , sep 2007.

[63] Julian L. Griffin and Denis V. Rubtsov. Analytical Methodology Standards for Metabolomics. In Encyclopedia of Spectroscopy and Spectrometry, pages 4-8. 2010.

[64] G. E. P. Box and D. R. Cox. An analysis of transformations. Journal of the Royal Statistical Society. Series B (Methodological, pages 211-252, 1964.

[65] Raymond J. Carroll and David Ruppert. Transformation and weighting in regression. Chapman and Hall, 1988.

[66] Bernard (Bernard A.) Rosner. Fundamentals of biostatistics. Brooks/Cole, Cengage Learning, 2011.

[67] Daniel J. Denis. Applied univariate, bivariate, and multivariate statistics.

[68] William S Noble. How does multiple testing correction work? Nature Biotechnology, 27(12):1135-1137, dec 2009.

[69] Barbara G. Tabachnick and Linda S. Fidell. Using multivariate statistics. Pearson Education, 2013.

[70] Richard A. (Richard Arnold) Johnson and Dean W. Wichern. Applied multivariate statistical analysis. Prentice Hall, 2002.

[71] Douglas M. Hawkins*. The Problem of Overfitting. 2003. 
[72] Open Science Collaboration. Estimating the reproducibility of psychological science. Science, 349(6251):aac4716-aac4716, aug 2015.

[73] C. Glenn Begley and Lee M. Ellis. Drug development: Raise standards for preclinical cancer research. Nature, 483(7391):531-533, mar 2012.

[74] John P. A. Ioannidis and Muin J. Khoury. Improving Validation Practices in Omics Research. Science, 334(6060), 2011.

[75] Kim DG G van de Kant, Linda JTM T M van der Sande, Quirijn Jöbsis, Onno CP P van Schayck, and Edward Dompeling. Clinical use of exhaled volatile organic compounds in pulmonary diseases: a systematic review. Respiratory research, 13(1):117, dec 2012.

[76] Anders Christiansen, Jesper Rømhild Davidsen, Ingrid Titlestad, Jørgen Vestbo, and Jan Baumbach. A systematic review of breath analysis and detection of volatile organic compounds in COPD. Journal of Breath Research, 10(3):034002, aug 2016.

[77] J. Cavaleiro Rufo, J. Madureira, E. Oliveira Fernandes, and A. Moreira. Volatile organic compounds in asthma diagnosis: a systematic review and meta-analysis. Allergy, 71(2):175188 , feb 2016.

[78] Lajos Pusztai, Christos Hatzis, and Fabrice Andre. Reproducibility of research and preclinical validation: problems and solutions. Nature Reviews Clinical Oncology, 10(12):720-4, oct 2013.

[79] L Blanchet, A Smolinska, A Baranska, et al. Factors that influence the volatile organic compound content in human breath. J Breath Res, 11(1):16013, feb 2017.

[80] Andras Bikov, Koralia Paschalaki, Ron Logan-Sinclair, et al. Standardised exhaled breath collection for the measurement of exhaled volatile organic compounds by proton transfer reaction mass spectrometry. BMC pulmonary medicine, 13:43, jul 2013.

[81] Kaisa J. Raninen, Jenni E. Lappi, Maria L. Mukkala, et al. Fiber content of diet affects exhaled breath volatiles in fasting and postprandial state in a pilot crossover study. Nutrition Research, 36(6):612-619, jun 2016.

[82] Agnieszka Baranska, Ettje Tigchelaar, Agnieszka Smolinska, et al. Profile of volatile organic compounds in exhaled breath changes as a result of gluten-free diet. Journal of breath research, $7(3): 037104$, sep 2013.

[83] Samuel V Angiuoli, Aaron Gussman, William Klimke, et al. Toward an online repository of Standard Operating Procedures (SOPs) for (meta)genomic annotation. Omics : a journal of integrative biology, 12(2):137-41, jun 2008.

[84] Patrizia Bernini, Ivano Bertini, Claudio Luchinat, et al. Standard operating procedures for pre-analytical handling of blood and urine for metabolomic studies and biobanks. Journal of Biomolecular NMR, 49(3-4):231-243, apr 2011.

[85] Paola Gramatica. Principles of QSAR models validation: internal and external. QSAR \& Combinatorial Science, 26(5):694-701, may 2007.

[86] Gary S. Collins, Johannes B. Reitsma, Douglas G. Altman, and Karel G.M. Moons. Transparent Reporting of a multivariable prediction model for Individual Prognosis Or Diagnosis (TRIPOD): The TRIPOD Statement. Annals of Internal Medicine, 162(1):55, jan 2015.

[87] S. K. Brown, M. R. Sim, M. J. Abramson, and C. N. Gray. Concentrations of Volatile Organic Compounds in Indoor Air - A Review. Indoor Air, 4(2):123-134, jun 1994.

[88] Christian Monn. Exposure assessment of air pollutants: a review on spatial heterogeneity and indoor/outdoor/personal exposure to suspended particulate matter, nitrogen dioxide and ozone. Atmospheric Environment, 35(1):1-32, jan 2001.

[89] J Kwak and G Preti. Volatile disease biomarkers in breath: a critique. Curr Pharm Biotechnol, 12(7):1067-1074, 2011.

[90] A Tangerman, M T Meuwese-Arends, and J B Jansen. Cause and composition of foetor hepaticus. Lancet (London, England), 343(8895):483, feb 1994.

[91] M R Wills and J Savory. Biochemistry of renal failure. Annals of clinical and laboratory science, 11(4):292-9. 
[92] L Laffel. Ketone bodies: a review of physiology, pathophysiology and application of monitoring to diabetes. Diabetes/metabolism research and reviews, 15(6):412-426, 1999.

[93] T C Vary, J H Siegel, T Nakatani, T Sato, and H Aoyama. A biochemical basis for depressed ketogenesis in sepsis. J Trauma, 26(5):419-425, 1986.

[94] C M Kneepkens, Guy Lepage, Claude C. Roy, et al. The potential of the hydrocarbon breath test as a measure of lipid peroxidation. Free Radical Biology and Medicine, 17(2):127-60, aug 1994.

[95] Meggie Hakim, Yoav Y. Broza, Orna Barash, et al. Volatile organic compounds of lung cancer and possible biochemical pathways. Chemical Reviews, 112(11):5949-5966, nov 2012.

[96] M Kanehisa and S Goto. KEGG: kyoto encyclopedia of genes and genomes. Nucleic acids research, 28(1):27-30, jan 2000.

[97] Marjolein Drent, Elyse E. Lower, and Jolanda De Vries. Sarcoidosis-associated fatigue. European Respiratory Journal, 40(1):255-263, jul 2012.

[98] M A Spruit, M J Thomeer, R Gosselink, et al. Skeletal muscle weakness in patients with sarcoidosis and its relationship with exercise intolerance and reduced health status. Thorax, 60(1):32-38, jan 2005.

[99] David R Park. The Microbiology of Ventilator-Associated Pneumonia Features of Specific Common VAP Pathogens The Prevalence of Routine Bacterial Pathogens in VAP VAP in Patients After Tracheotomy VAP in Patients With Traumatic Injuries. Respiratory care, 50(6):742-765, jun 2005.

[100] Stefan Schulz and Jeroen S Dickschat. Bacterial volatiles: the smell of small organisms. Natural product reports, 24(4):814-842, aug 2007.

[101] Heather D Bean, Jean-Marie D Dimandja, and Jane E Hill. Bacterial volatile discovery using solid phase microextraction and comprehensive two-dimensional gas chromatographytimeof-flight mass spectrometry. Journal of chromatography. B, Analytical technologies in the biomedical and life sciences, 901:41-46, jul 2012.

[102] J. N. Labows, K. J. McGinley, G. F. Webster, and J. J. Leyden. Headspace analysis of volatile metabolites of Pseudomonas aeruginosa and related species by gas chromatographymass spectrometry. Journal of Clinical Microbiology, 12(4):521-526, oct 1980.

[103] James M. Zechman, Scott Aldinger, and John N. Labows. Characterization of pathogenic bacteria by automated headspace concentration-gas chromatography. Journal of Chromatography B: Biomedical Sciences and Applications, 377(C):49-57, jan 1986.

[104] Robin Michael Statham Thorn and John Greenman. Microbial volatile compounds in health and disease conditions. Journal of Breath Research, 6(2):024001, jun 2012.

[105] Sasidhar Maddula, Lars Mathias Blank, Andreas Schmid, and J??rg Ingo Baumbach. Detection of volatile metabolites of Escherichia coli by multi capillary column coupled ion mobility spectrometry. Analytical and Bioanalytical Chemistry, 394(3):791-800, jun 2009.

[106] Randall A. Allardyce, Alex L. Hill, and David R. Murdoch. The rapid evaluation of bacterial growth and antibiotic susceptibility in blood cultures by selected ion flow tube mass spectrometry. Diagn. Microbiol. Infect. Dis., 55(4):255, aug 2006.

[107] Jae Sung Pyo, Hyun Kyoung Ju, Jeong Hill Park, and Sung Won Kwon. Determination of volatile biomarkers for apoptosis and necrosis by solid-phase microextraction-gas chromatography/mass spectrometry: A pharmacometabolomic approach to cisplatin's cytotoxicity to human lung cancer cell lines. Journal of Chromatography B, 876(2):170-174, dec 2008.

[108] Margaret A. Lawlor and Dario R. Alessi. PKB/Akt. Journal of Cell Science, 114(16), 2001.

[109] Wei Han, Joell J Gills, Regan M Memmott, Stephen Lam, and Phillip A Dennis. The chemopreventive agent myoinositol inhibits Akt and extracellular signal-regulated kinase in bronchial lesions from heavy smokers. Cancer prevention research (Philadelphia, Pa.), 2(4):370-6, apr 2009.

[110] Gregory J. Hannon. RNA interference. Nature, 418(6894):244-251, jul 2002.

[111] Jennifer A. Doudna and Emmanuelle Charpentier. The new frontier of genome engineering with CRISPR-Cas9. Science, 346(6213), 2014. 
Nederlandse Samenvatting 
Vluchtige organische verbindingen (VOCs) worden gedefinieerd als koolstofverbindingen die gemakkelijk verdampen bij kamertemperatuur. Ze kunnen verdeeld worden in verschillende groepen op basis van hun chemische eigenschappen. VOCs worden geproduceerd door bronnen van exogene (buiten het lichaam) of endogene (binnen het lichaam) oorsprong. Exogene VOCs worden onder andere geproduceerd door vervuiling, planten, voedsel en schimmels. Endogene VOCs worden geproduceerd door het menselijk lichaam zelf of door bacteriën die zich in het lichaam bevinden.

Uitademingslucht bevat naast stikstof, zuurstof en andere gassen ook VOCs. Deze uitgeademde VOCs kunnen zowel van endogene als exogene bronnen komen. Endogene VOCs worden geproduceerd in een weefsel of bacterie in het lichaam en worden getransporteerd naar de longen, waarna ze worden uitgeademd. Exogene VOCs komen binnen via de longen, huid, of ingewanden en worden na eventueel gebruik ook getransporteerd naar de longen, waarna ze worden uitgeademd.

Wanneer de concentraties van VOCs in de uitademingslucht worden vergeleken tussen een ziek en gezond persoon kunnen verschillen worden ontdekt die een indicatie geven over de aan- of afwezigheid van ziekte. Het doel van dit proefschrift was om de verschillende aspecten van het meten van VOCs in de uitademingslucht te onderzoeken. Ten behoeve van dit doel zijn zeven studies beschreven in dit proefschrift om de volgende doelen te behalen: (i) het beoordelen van de huidige literatuur over het verwerken en analyseren van de verkregen data in het onderzoek naar uitademingslucht, (ii) het onderzoeken van de potentie van de analyse van uitademingslucht ter diagnose van verschillende longziektes, (iii) het testen van de kwaliteit en betrouwbaarheid van de onderscheidende VOCs, (iv) het onderzoeken van de bruikbaarheid van VOCs analyse in vitro in cellen en bacteriën, en (v) het identificeren van aannemelijke endogene bronnen van VOCs in vitro.

\section{Belangrijkste bevindingen}

\section{De analyse van uitademingslucht kan onderscheid maken tussen ernstig zieke patiënten met en zonder ventilator geassocieerde pneumonie}

Ventilator geassocieerde pneumonie (VAP) is een infectieziekte die voorkomt in de Intensive Care (IC). Om de ziekte vast te stellen moeten patiënten een zeer invasieve en risicovolle procedure genaamd bronchoalveolaire lavage ondergaan. In hoofdstuk 3 werd onderzocht of het mogelijk was om de aanwezigheid van de ziekte vast te stellen met behulp van uitademingslucht. Hiervoor is de uitademingslucht van 100 patiënten opgevangen en geanalyseerd. Uit de resultaten is gebleken dat het mogelijk was om een onderscheid te maken tussen patiënten die VAP ontwikkelden en patiënten waarbij dat niet gebeurde. Dit onderscheid was vast te stellen met een nauwkeurigheid van $74.2 \%$ en was gebaseerd op basis van een profiel van 12 VOCs. Door middel van eerder vergaarde kennis werd voor een aantal van deze onderscheidende VOCs een potentiele endogene bron vastgesteld.

Op basis van deze bevindingen kon worden geconcludeerd dat uitademingslucht 
potentieel een belangrijke rol kan gaan spelen in de diagnose van VAP. Het heeft veel voordelen ten opzichte van de huidige gouden standaard, bronchoalveolaire lavage, vanwege het snelle en non-invasieve karakter van de methode. Daarnaast kan het de opmars van antibiotica resistentie remmen door snellere diagnose, waardoor eerder gehandeld kan worden wanneer een longontsteking wordt vastgesteld.

\section{Met behulp van uitademingslucht kan een onderscheid gemaakt worden tussen drie verwante chronische longziekten, maar de resultaten zijn mogelijk niet reproduceerbaar}

De term Interstitiële longaandoeningen (ILD) betreft een groep longaandoeningen met onder andere longfibrose (IPF) en bindweefselziekte-geassocieerde interstitiële longziekte (CTD-ILD). De combinatie van de generieke symptomen en de lage incidentie en prevalentie van deze ziektes bemoeilijkt het vinden van de juiste diagnose. Voor de patiënt resulteert dit in een lange periode van onzekerheid voor een diagnose gesteld kan worden. In hoofdstuk 4 is onderzocht of het onderscheid gemaakt kon worden tussen 51 IPF en 53 CTD-ILD patiënten. Een profiel van 16 VOCs werd ontdekt dat kon voorspellen welke ziekte een patiënt had met een nauwkeurigheid van $76.9 \%$. Dit resultaat suggereert deze uitgeademde VOCs samenhangen met de onderliggende pathofysiologische mechanismen van die specifieke ziekte. Daarnaast was het mogelijk om beide groepen patiënten te onderscheiden van een groep gezonde vrijwilligers. Hoewel deze bevindingen veelbelovend waren, was het niet mogelijk om de studie te valideren, vermoedelijk vanwege de heterogene aard van ILD in combinatie met het relatief lage aantal patiënten in deze studie.

Op basis van de bevindingen in deze studie kan worden vastgesteld dat patiënten met ILD een ander VOC-profiel uitademen dan gezonde personen, maar ook dat verschillende vormen van ILD te onderscheiden zijn van elkaar. Toch is gebleken dat het aantal patiënten in deze studie niet toereikend was, waardoor een grotere studie nodig is om de waarde van de onderscheidende VOCs te kunnen bepalen.

\section{Externe studie validatie is essentieel om de reproduceer- baarheid van een klinische studie te waarborgen}

Gedurende het laatste decennium is een grote hoeveelheid klinische studies gepubliceerd waarbij VOCs in uitademingslucht zijn gebruikt om een ziekte te diagnosticeren. Wanneer deze studies worden vergeleken is duidelijk te zien dat er weinig overlap is tussen de onderscheidende VOCs in de verschillende studies. Er zijn een aantal oorzaken voor dit probleem, waarvan de voornaamste het gebrek aan validatie van de studie is. Het doel van hoofdstuk $\mathbf{5}$ was om het effect van verschillende vormen van validatie te testen op data van een klinische studie. In deze studie werd een profiel van negen VOCs gevonden dat onderscheid kon maken tussen patiënten met sarcoïdose, een vorm van ILD, met een nauwkeurigheid van $79.4 \%$. Wanneer verschillende vormen van validatie werden vergeleken bleek dat de leave-k-out validatie de meest optimistische voorspelling deed, terwijl de onafhankelijke externe validatie de meest realistische voorspelling gaf. 
Uit deze studie kan geconcludeerd worden dat onafhankelijke externe validatie van groot belang is om de betrouwbaarheid van een klinische studie vast te stellen. We raden daarom aan om deze vorm van validatie altijd toe te passen in een klinische studie waarin de analyse van uitademingslucht wordt toegepast.

\section{Het is mogelijk VOCs te detecteren die zijn uitgescheiden door bacteriën in vitro}

Naast het gebrek aan validatie in klinische studies is de kennis over de biologische oorsprong van VOCs zeer summier. Toch is het cruciaal deze kennis te vergroten om niet alleen te begrijpen waarom bepaalde VOCs uitgeademd worden in een bepaalde ziekte, maar ook om uitademingslucht te kunnen toepassen voor ziektebewaking. Hoofdstuk 6 trachtte te achterhalen welke VOCs werden uitgescheiden door verschillende soorten bacteriën die vaak infecties veroorzaken in de longen. In deze studie werden 6 soorten bacteriën gekweekt, waarna de lucht boven de bacteriën werd geanalyseerd om veranderingen in de productie van VOCs op te sporen. Vier soorten bacteriën konden worden onderscheiden met behulp van een profiel van 25 VOCs. Vijftien van deze VOCs waren specifiek voor $n$ soort bacterie, terwijl de overige tien werden geproduceerd door meerdere soorten. Naast deze resultaten kon een onderscheid gemaakt worden tussen Meticilline Resistente Staphylococcus aureus (MRSA) en Meticilline Gevoelige Staphylococcus aureus (MSSA) op basis van 8 VOCs. Verscheidene VOCs konden terug geleid worden naar een specifiek onderliggend proces, zoals de koolwaterstoffen die voortkomen uit lipide peroxidatie. Een groot aandeel van de overige VOCs kon niet gekoppeld worden aan een specifiek proces, maar werd gevonden in andere studies waarin dezelfde bacteriën werden onderzocht.

Uit deze bevindingen kon geconcludeerd worden dat verschillende soorten bacteriën een ander patroon aan VOCs uitscheiden. Hieruit kan worden afgeleid welke processen specifieke VOCs uitscheiden. Daarnaast was het mogelijk MRSA van MSSA te onderscheiden. Deze resultaten kunnen in de toekomst vertaald worden naar een klinische toepassing waar het mogelijk is bij de diagnose van een longinfectie vast te stellen welke bacterie het veroorzaakt. Wanneer dit mogelijk is kan de juiste antibiotica gekozen worden, waardoor genezing sneller plaatsvindt en verdere antibiotica resistentie bestreden kan worden.

\section{Blootstelling aan een giftige stof resulteert in verandering in de uitscheiding van VOCs door longcellen in vitro}

Niet alleen bacteriën, maar ook menselijke cellen kunnen VOCs produceren. Ook op dit vlak is er een gebrek aan kennis over de biologische oorsprong. Daarnaast is het identificeren van ziekte een belangrijke doelstelling voor de analyse van uitademingslucht. In hoofdstuk 7 zijn cellen in vitro blootgesteld aan twee giftige stoffen om te achterhalen of cellen andere VOC profielen produceren wanneer er schade ontstaat door deze giftige stoffen. In beide gevallen was het mogelijk de beschadigde cellen te onderscheiden van de gezonde cellen. Omdat het werkingsmechanisme van beide giftige stoffen bekend is, was het mogelijk om een link te leggen tussen specifieke processen en de onderscheidende VOCs. Toch was er, ondanks dat beide stoffen een 
soortgelijk werkingsmechanisme hadden, maar n VOC die werd gevonden in beide experimenten. Dit kan veroorzaakt worden door verschillende factoren, maar het is aannemelijk dat de voornaamste oorzaak het verschil in blootstellingstijd is.

Uit deze resultaten kan worden geconcludeerd dat ook menselijke cellen andere VOCs uitscheiden, in dit geval na blootstelling aan giftige stoffen. Deze resultaten kunnen mogelijk in de toekomst vertaald worden naar een klinische studie waarbij bijvoorbeeld gekeken wordt naar de relatie tussen blootstelling aan sigaretten en de samenstelling van VOCs in de uitademingslucht. Daarnaast zijn relaties ontdekt tussen de uitgescheiden VOCs en de werkingsmechanismen van de giftige stoffen. Deze relaties moeten gevalideerd worden met meer onderzoek, maar geven een duidelijk eerste inzicht in de biologische oorsprong van endogene VOCs.

\section{Door het combineren van VOCs en ander biologische bron- nen van data kunnen aannemelijke relaties worden vastgesteld tussen VOCs en de onderliggende biologische processen}

Uit een cel kunnen verschillende lagen van informatie verzameld worden. Ten eerste is informatie opgeslagen in het DNA in de celkern, genaamd genen. Om de cel in leven te houden worden op basis van deze informatie bouwstoffen gemaakt, genaamd eiwitten en enzymen. Daarna worden deze bouwstoffen gebruikt om energie te produceren of de samenstelling van de cel te onderhouden. Tijdens deze processen worden metabolieten geproduceerd, die daarna omgezet kunnen worden in VOCs. Om daadwerkelijk vast te stellen welke onderliggende biologische processen VOCs produceren zal informatie verzameld moeten worden van deze verschillende informatielagen. In hoofdstuk 8 is deze strategie gebruikt om informatie te verzamelen van de genen, metabolieten en VOCs. Deze informatie is samengevoegd en gebruikt om relaties te ontdekken tussen uitgescheiden VOCs en de onderliggende genen en metabolieten. Een voorbeeld hiervan is de VOC nonanal, die correleert met de metaboliet myoinositol. Dit metaboliet is gelinkt aan genen die betrokken zijn bij celdood-, DNA herstel- en kanker-gerelateerde processen. Het is daarom plausibel dat nonanal wordt geproduceerd door deze celdood-, DNA herstel- en kanker-gerelateerde processen.

Op basis van deze bevindingen kan geconcludeerd worden dat verschillende relaties zijn ontdekt tussen uitgescheiden VOCs en de onderliggende biologische processen, met nonanal als voorbeeld. Toch is het belangrijk te noemen dat associatie niet gelijk is aan causaliteit. Er is een grote behoefte aan in vitro onderzoek dat de causaliteit van deze associaties aantoont.

\section{Algemene conclusie}

Dit proefschrift heeft aangetoond dat, hoewel uitademingslucht veelvuldig is toegepast in de vorm van klinische studie, twee problemen moeten worden opgelost voor een klinische toepassing mogelijk is. Ten eerste heeft dit proefschrift het belang van geschikte (bij voorkeur externe) validatie aangetoond die nodig is om de betrouwbaarheid en reproduceerbaarheid van een klinische studie te waarborgen. Ten tweede, het vergaren en uitbreiden van de biologische kennis onderliggend aan de endogene productie van 
VOCs is van groot belang. Dit proefschrift heeft de eerste stap gezet in het achterhalen waar specifieke VOCs vandaan komen, maar heeft voornamelijk associaties gevonden. Het is van belang om causaliteit vast te stellen in toekomstige in vitro experimenten. In het algemeen is een collectieve inspanning nodig vanuit de gemeenschap die betrokken is bij het onderzoek naar uitademingslucht om deze twee problemen op te lossen, zodat deze non-invasieve methode in de toekomst geïmplementeerd wordt in de kliniek. 
Valorisatie-addendum 
Wereldwijd lijden meer dan een miljard mensen aan een longziekte. Daarnaast veroorzaken de drie meest voorkomende long ziekten 9.5 miljoen sterfgevallen jaar, een-zesde van het totaal aantal doden. De bijbehorende jaarlijkse zorgkosten en arbeidsverzuimkosten bedragen alleen al in Nederland een totaal van $€ 3.6$ miljard, met naar schatting een bedrag van $€ 379.6$ miljard wereldwijd. Naast het behandelen van de ziekte is een vroege diagnose zeer belangrijk. Een vroege diagnose in combinatie met de juiste medicatie resulteert in een kleiner aandeel van ernstig zieke patinten, waardoor en de kwaliteit van leven van de patinten hoger wordt en bespaard kan worden op de zorgkosten en arbeidsverzuimkosten.

Op dit moment worden voornamelijk invasieve methoden gebruikt voor het stellen van een diagnose, vaak na een lang traject van de eerste symptomen tot de definitieve uitslag. Dit kan een grote impact hebben op de patiënt door de lichamelijke en mentale belasting en de risicos die horen bij invasieve diagnostiek. Voorbeelden hiervan zijn het gebruik van biopsies ter diagnose van sarcodose en bronchoalveolaire lavage ter bepaling van bacteriële longinfecties in de intensive care (IC). In tegenstelling tot deze invasieve methoden is de analyse van uitademingslucht juist zeer non-invasief. Deze methode vereist alleen dat een patiënt rustig uitademt, waarna de diagnose snel gesteld kan worden. Het gebruik van deze methode resulteert in een substantiële verlaging van de lichamelijke en geestelijke belasting van de patiënt en is kosteneffectiever dan de huidige gebruikte diagnostische methoden.

Het onderzoek in dit proefschrift is voornamelijk van belang voor medisch specialisten, ziekenhuizen en patiënten. Daarnaast kunnen zorgverzekeraars en de overheid baat hebben bij deze gepresenteerde resultaten.

Ten eerste is het opvangen van uitademingslucht een simpele methode die weinig training vereist. Dit is van belang voor de medisch specialisten, die bijvoorbeeld tijdens een intakegesprek meteen uitademingslucht kunnen verzamelen om een snelle diagnose te stellen. Daarnaast kan deze methode, in tegenstelling tot invasieve methoden zoals biopsies en bronchoalveolaire lavage, gebruikt worden bij kinderen, ouderen en kwetsbare patiënten die de invasieve diagnostiek niet kunnen ondergaan. Zelfs als een fractie van de invasieve diagnostiek vervangen kan worden door de analyse van uitademinglucht, levert dit voor de patiënten een grote lastenverlichting op in de vorm van minder onzekerheid en een hogere kwaliteit van leven. Bovendien zullen artsen vaker en sneller toegang hebben tot relevante informatie over de ziekte, zoals bijvoorbeeld de aanwezigheid van exacerbaties, de effectiviteit van medicatie en de algemene voortgang van de ziekte.

Ten tweede, invasieve methoden behoeven vaak grote hoeveelheden aan medisch materieel en manuren. Voor de analyse van uitademingslucht zijn substantieel minder kostbare materialen en apparatuur nodig, en zijn minder manuren vereist dan bij invasieve technieken als biopsies. Dit is ook gunstig voor zorgverzekeraars en de overheid doordat aan de zorg gespendeerde kosten gereduceerd kunnen worden. Tenslotte, de belangrijkste belangengroep is de patiënten zelf. De patiënten zullen door het klinisch gebruik van uitademingslucht minder gebruik hoeven maken van invasieve diagnostiek, met als gevolg een hogere kwaliteit van leven. Hoewel de patiënten zelf geen actieve rol kunnen spelen in het vertalen van de methode naar een klinische toepassing, kunnen patiëntenverenigingen met behulp van dit onderzoek de discussie aan gaan met ziekenhuizen en daardoor in de implementatie van de methode stimuleren. 
In dit proefschrift in het bijzonder ligt de nadruk op twee uitdagingen die naar voren zijn gekomen in de klinische studies. Ten eerste is in het laatste decennium een grote hoeveelheid klinische studies gepubliceerd, maar het ontbreekt aan voldoende interne en externe validatie van de resultaten. Dit proefschrift toont aan dat externe validatie essentieel is en spoort aan tot meer samenwerkingen en validatie binnen en tussen onderzoeksgroepen. Ten tweede, de biologische oorsprong van de organische verbindingen in de uitademingslucht is, met een enkele uitzondering, onbekend. Toch is deze kennis van belang om niet alleen ziekte te kunnen diagnosticeren, maar ook voor ziektebewaking en om de effectiviteit van medicatie te kunnen bepalen. Dit proefschrift heeft de eerste stap gezet in het ontrafelen van de biologische oorsprong van deze verbindingen.

De bevindingen van het onderzoek beschreven in dit proefschrift kunnen tevens vertaald worden naar een concrete toepassing. De predictiemodellen gegenereerd voor de studies in dit proefschrift kunnen namelijk gebruikt worden ter diagnostiek in de kliniek. Daarnaast zou deze methode toegepast kunnen worden in eHealth applicaties. Zo kan bijvoorbeeld een apparaat ontwikkeld worden dat de uitademingslucht opvangt en analyseert. Dit apparaat kan gekoppeld worden aan de smartphone, waarbij de arts de ziekteontwikkeling op afstand kan waarnemen. Bovendien kan de methode toegepast worden ten behoeve van de vroege opsporing van de juist bacterie in longinfecties in de IC om zo sneller de juiste antibiotica toe te dienen, waardoor de oprukkende antibiotica resistentie kan worden tegengegaan.

Predictiemodellen voor ziekten als inflammatoire darmziekten (IBD), hepatitis, chronische obstructieve longziekte (COPD) en ventilator-geassocieerde pneumonie (VAP) bestaan al. Deze selectie kan door dit proefschrift uitgebreid worden met sarcodose, longfibrose en bindweefselziekte.

Naast de klinische toepassing van de methode ter diagnose van ziektes besteedt het proefschrift ook aandacht aan de biologische relevantie van de organische verbindingen in de uitademingslucht die gebruikt worden voor de diagnose. De resultaten van dit proefschrift zijn een eerste stap in het vergaren van meer kennis over de onderliggende biologische processen waardoor het mogelijk is diagnoses te stellen met behulp van uitademingslucht. Het zal daarom onderzoekers stimuleren deze mechanismen te ontrafelen. Als dit wordt opgelost zal het in de toekomst mogelijk zijn Om naast diagnose ook het verloop van de ziekte te bewaken met behulp van uitademingslucht. Dit maakt het mogelijk om sneller medicatie bij te stellen, waardoor de belasting voor de patiënten en de kosten verder verminderd kunnen worden. 

Dankwoord 
Dit proefschrift was niet tot stand gekomen zonder de hulp en het vertrouwen van velen. Mijn speciale dank gaat uit naar

Promotor

Frederik Jan van Schooten

Voor het vertrouwen in mijn kunnen

Voor het enthousiasme voor nieuwe ideeën

Voor het stimuleren om een kans bij Maastro te nemen

Co-promotor

\section{Agi Smolinska}

For always having my back

For your determination to do things the right way

For inspiring me to become a scientist like you

\section{Co-promotor}

\section{Agnes Boots}

Voor je eerlijke mening

Voor je kritische blik

En al dat leuke mail verkeer

Paranimfen

Alie

Voor de gezellige tijd in de eerste helft van mijn $\mathrm{PhD}$

Voor alle steun daarna

\section{Charlotte}

Voor je heerlijke sarcastische humor

Voor het aanhoren van al mijn problemen

En voor die overheerlijke eieren

(Ex-)Collega's bij Farmacologie en Toxicologie

For the good times we had doing research

(and everything that was not research)

Phillippe, Timme, Kristien, Matt, Carmen, Misha, Mireille, Ming, Quan, Sven, Gesiele, Caroline

In het bijzonder

Marvin, Roger, Jan, Daniëlle, Marie-Claire

Collega's van andere afdelingen

Voor de leuke samenwerking

Marjolein, Remy

In het bijzonder

Ronny, Egon

Mijn Maastro collega's 
Voor het vertrouwen en de gezelligheid

Sander, Tim L., Tim H., Peter, Johan

In het bijzonder

Natascha en Andre

Mijn lieve familie

Mam, Pap, Mart, Lisa,

Ingrid, Ad, Kiki, Rik,

Ooms en Tantes,

Oma's en Opa's

Voor alle steun en interesse in mijn onderzoek,

ook al is het soms moeilijk te begrijpen

Mijn grote liefde

\section{Bob}

Voor het grenzeloze vertrouwen

en je onvoorwaardelijke liefde,

ook al word je soms gek van mijn gestress

Mijn grote kleine schat

Jake

Voor je liefde, kusjes en knuffels

Tenslotte wil ik deze thesis opdragen

aan een persoon in het bijzonder

Pap

Bedankt dat je me altijd hebt aangespoord om het beste uit mezelf te halen

Bedankt voor al die museumbezoekjes en intellectuele gesprekken

Bedankt voor je immense invloed op de persoon die ik ben geworden

En natuurlijk bedankt voor de prachtige kaft 

Curriculum Vitae 
Rianne Rebecca Roger Fijten was born on April 15th 1990 in Voorburg, the Netherlands. In September 2008, she enrolled in the bachelor Biomedical Sciences at Maastricht University, formerly known as Molecular Life Sciences. After graduating, she enrolled in the master's degree Biomedical Sciences at Maastricht University in 2011. During that period she built her expertise in bioinformatics at Imperial College London, UK. In July 2013, she started a PhD at the department of Toxicology at Maastricht University, where she applied her previously acquired bioinformatics skills to investigate the use of Volatile Organic Compounds in exhaled breath for the diagnosis of lung diseases. During this four year period, she attended a variety of international conferences and won several prizes for her (poster) presentations. Additionally, she was involved in various teaching activities including lecturing, practical supervision, leading problem-based learning groups and grading reports. Other activities included management and organization of a practical, the NUTRIM symposium, and chairing the NUTRIM PhD students council. During the last year of her PhD, she started working part-time in order to finish her $\mathrm{PhD}$ while working as a medical software engineer at Maastro Clinic. After finishing her $\mathrm{PhD}$ thesis, she started working as a postdoctoral researcher at Maastro Clinic. 
List of Publications 
1. Fijten R.R.R., Smolinska A., Boots A.W., Pachen D.M., Dallinga J.W. \& van Schooten F.J. (2017). Determining the endogenous sources of Volatile Organic Compounds excreted after benzo[a]pyrene exposure in vitro. PLOS Computational Biology. (Submitted)

2. Fijten R.R.R., Smolinska A., Drent M., Dallinga J., Mostard R., Pachen D., van Schooten F.J. \& Boots A.W. (2017). The necessity of external validation in exhaled breath research: a case study of sarcoidosis. Journal of Breath Research. (Accepted)

3. Fijten R.R.R., Smolinska A., Shi Q., Pachen D., Dallinga J., Boots A.W. \& van Schooten F.J. (2017). Exposure to genotoxic compounds alters in vitro cellular VOC excretion. Journal of Breath Research. (Accepted)

4. Shi Q., Fijten R.R.R., Spina D., Riffo Vasquez Y., Arlt V. M., Godschalk R. W., \& Van Schooten F.J. (2017). Altered gene expression profiles in the lungs of benzo[a]pyrene-exposed mice in the presence of lipopolysaccharide-induced pulmonary inflammation. Toxicology and Applied Pharmacology, 336.

5. Fijten R.R.R., Schnabel R., Smolinska A., Dallinga J., Boumans M.-L., Stobberingh E., van Schooten F. J. (2015). Analysis of volatile organic compounds in exhaled breath to diagnose ventilator-associated pneumonia. Scientific Reports, 5(October), 17179. (Shared first authorship)

6. Smolinska A., Hauschild A.C., Fijten R.R.R., Dallinga J.W., Baumbach J. \& van Schooten F.J. (2014). Current breathomics - a review on data pre-processing techniques and machine learning in metabolomics breath analysis. Journal of Breath Research. 8(2), 027105.

7. Boots A.W., Smolinska A., van Berkel J.J., Fijten R.R.R., Stobberingh E.E, Boumans M.L, Moonen E.J., Wouters E.F., Dallinga J.W. \& Van Schooten F.J. (2014). Identification of microorganisms based on headspace analysis of volatile organic compounds by gas chromatography-mass spectrometry. Journal Of Breath Research, 8(2), 027106.

8. Fijten, R.R.R., Jennen, D. G. J., \& van Delft, J. H. M. (2013). Pathways for ligand activated nuclear receptors to unravel the genomic responses induced by hepatotoxicants. Current Drug Metabolism, 14(10), 10221028. 